\title{
Targeting MDM2, the antagonist of the tumor suppressor p53
}

\section{Dissertation}

for the award of the degree

"Doctor of Philosophy (Ph.D.)"

within the doctoral program Molecular Medicine

of the Georg-August University School of Science (GAUSS)

at the Georg-August-Universität-Göttingen

Faculty of Medicine

submitted by

\section{Anusha Sriraman}

from Cochin, India

Göttingen 2018 


\section{Thesis Committee}

Prof. Dr. Matthias Dobbelstein, Institute of Molecular Oncology, University Medical Center Göttingen (UMG)

Prof. Dr. Steven Johnsen, Clinic for General, Visceral and Pediatric Surgery, University Medical Center Göttingen (UMG)

Prof. Dr. Heidi Hahn, Department of Human Genetics, University Medical Center Göttingen (UMG)

\section{Members of the Examination Board}

Referee: Prof. Dr. Matthias Dobbelstein, Institute of Molecular Oncology, University Medical Center Göttingen (UMG)

$2^{\text {nd }}$ Referee: Prof. Dr. Steven Johnsen, Clinic for General, Visceral and Pediatric Surgery, University Medical Center Göttingen (UMG)

\section{Additional members of the Examination Board}

Dr. Roland Dosch, Department of Developmental Biochemistry, University Medical Center Göttingen (UMG)

PD Dr. Halyna Shcherbata, Research Group - Gene Expression and Signaling, Max Planck Institute for Biophysical Chemistry (MPI-BPC)

Dr. Nuno Raimundo, Department of Cellular Biochemistry, University Medical Center Göttingen (UMG)

Date of oral examination: $10^{\text {th }}$ September 2018 


\section{AFFIDAVIT}

I hereby declare that the presented thesis entitled "Targeting MDM2, the antagonist of the tumor suppressor p53" has been written independently with no other sources and aids than quoted.

Gottingen, $20^{\text {th }}$ July 2018

Anusha Sriraman 
DEDICATION

This thesis is dedicated to Appa. 


\section{TABLE OF CONTENTS}

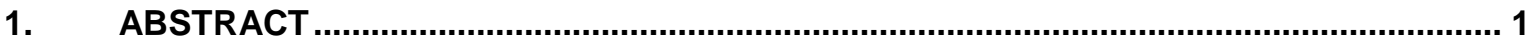

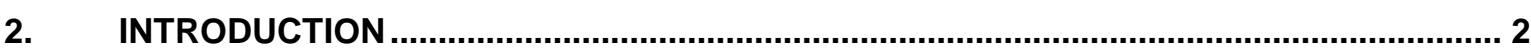

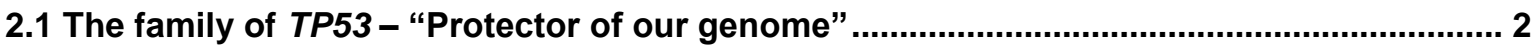

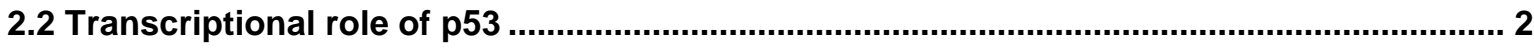

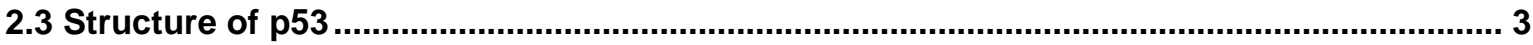

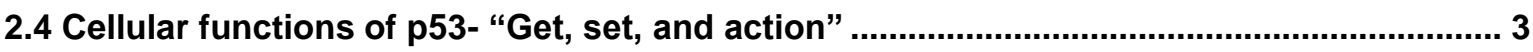

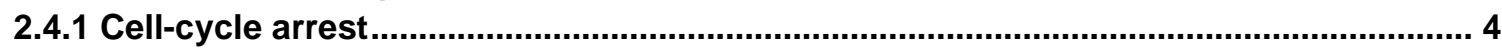

2.4.2 DNA repair

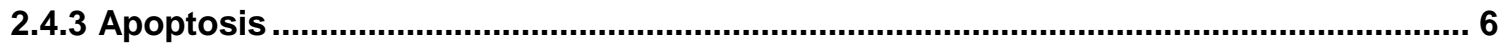

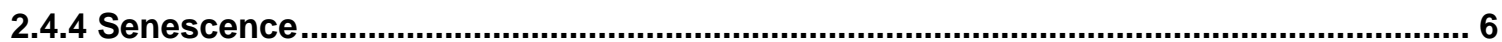

2.5 Post-translational modifications on p53

2.6 “Self-controlling p53"- MDM2

2.7 Structure of MDM2

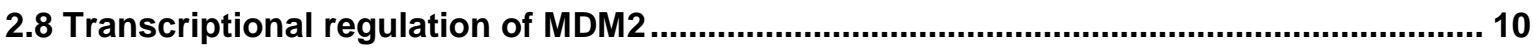

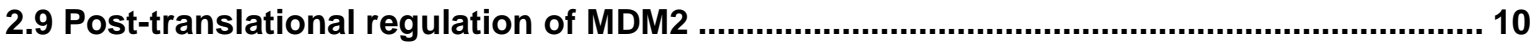

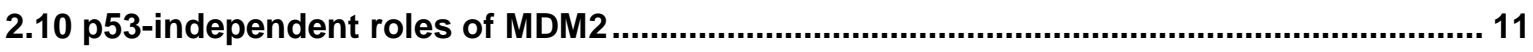

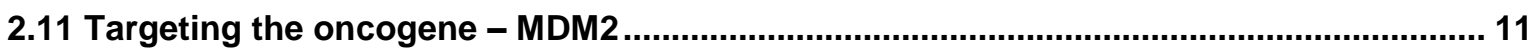

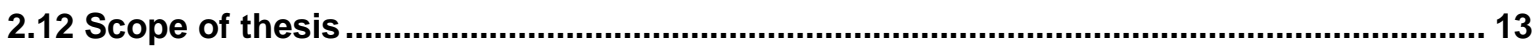

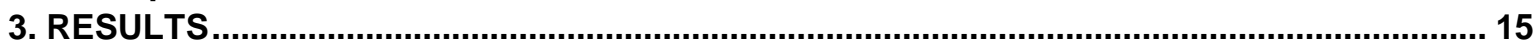

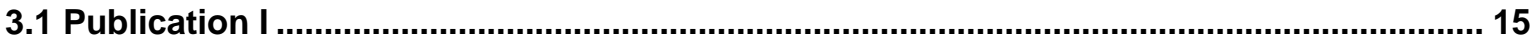

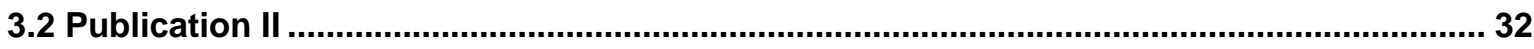

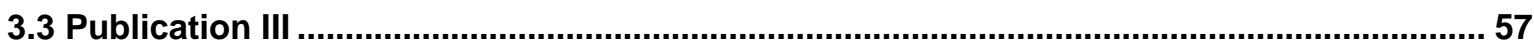

4. DISCUSSION

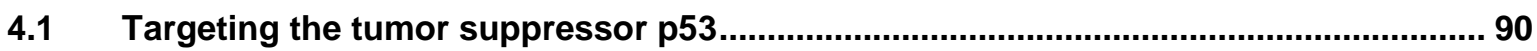

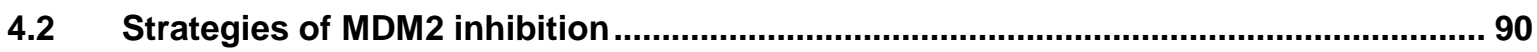

4.2.1 p53 accumulation protects cells against toxic effects of WEE1 inhibition ..........90

4.2.2 MDM2 and WIP1 inhibitors in combination are synergistic in activating p53...... 91

4.2.3 Antagonism of MDM2 and CDK4/6 inhibitors in sarcoma cells.......................... 92

4.3 Concerns regarding therapeutic inhibition of the MDM2-p53 interaction .................... 95

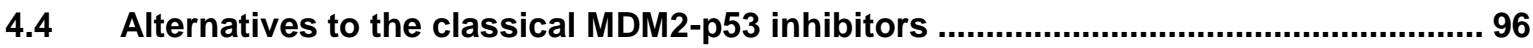

$4.5 \quad$ Harnessing mutant p53

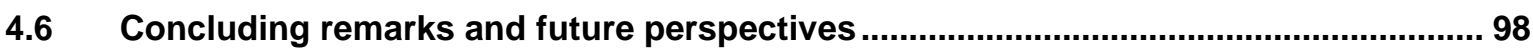

5. APPENDIX 


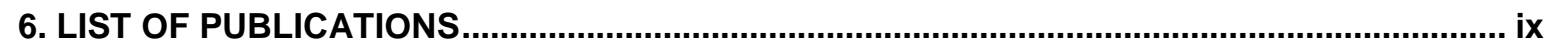

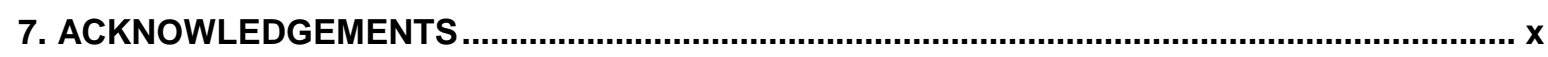

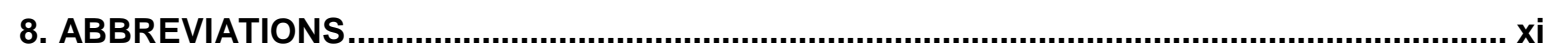

9. REFERENCES

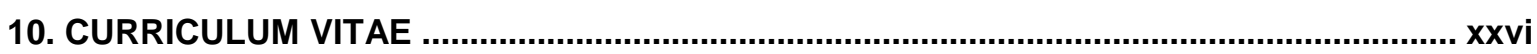




\section{LIST OF FIGURES}

FIGURE 21 SCHEMATIC STRUCTURE OF P53

FIGURE 22 ACTIVATION OF P53

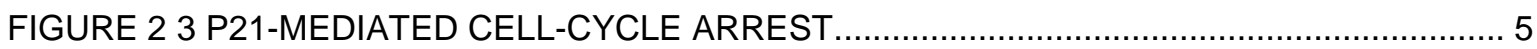

FIGURE 24 POST-TRANSLATIONAL MODIFICATIONS ON THE P53 PROTEIN. ........................ 7

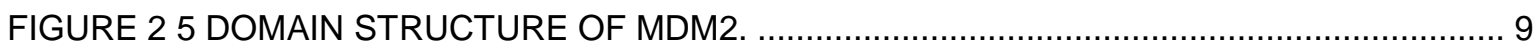

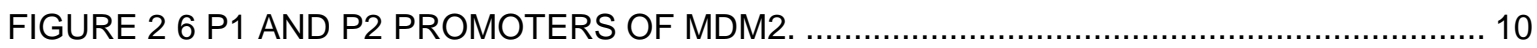

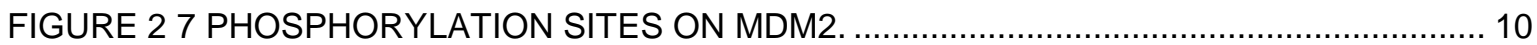

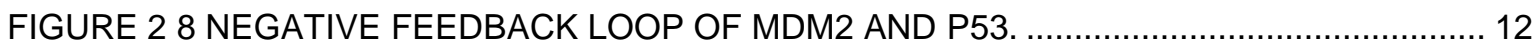

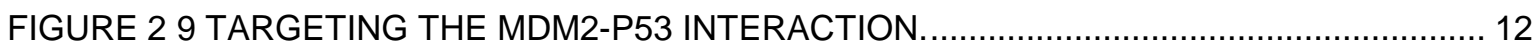

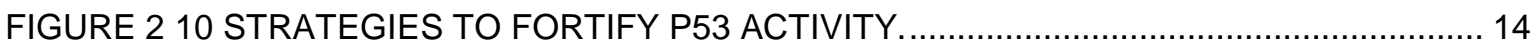

FIGURE 3.31 CDK4 INHIBITORS AND MDM2 ANTAGONISTS FAIL TO SYNERGIZE WITH

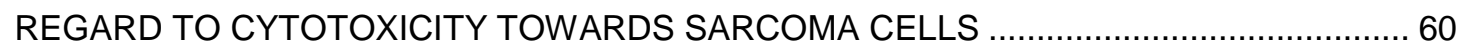

FIGURE 3.32 CDK4 IS REQUIRED FOR P53-INDUCED GENE EXPRESSION ........................... 62

FIGURE 3.33 SHORT-TERM REACTIVATION OF CDK4 IS SUFFICIENT TO RESCUE P53

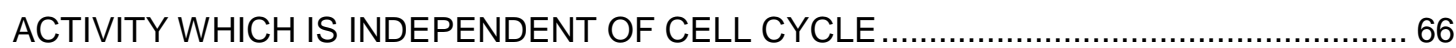

FIGURE 3.3 4 CDK4 INHIBITION ATTENUATES THE EXPRESSION OF A BROAD RANGE OF

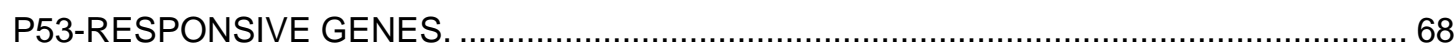

FIGURE 3.3 5 ASSOCIATION OF THE CDK4/CYCLIN D1 COMPLEX AND THE P53/MDM2

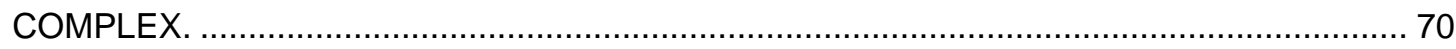

FIGURE 3.36 CDK4 INHIBITION DOES NOT INTERFERE WITH P53 BINDING TO ITS

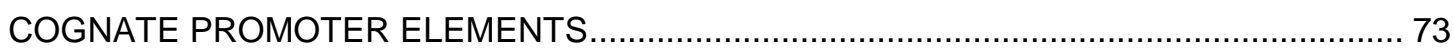

FIGURE 3.3 S 1 COMBINED TREATMENT OF NUTLIN WITH PD0332991 REVEALED ANTAGONISTIC EFFECTS WITH REGARD TO CELL VIABILITY

FIGURE 3.3 S 2 ALTERNATE CDK4/6 INHIBITORS AND MDM2 ANTAGONISTS DECREASE P53 TARGET GENE EXPRESSION IN VARIOUS SARCOMA CELL LINES. ........................ 85

FIGURE 3.3 S 3 IMPAIRED COMPLEX FORMATION OF CDK4 WITH CYCLIN D1 UPON

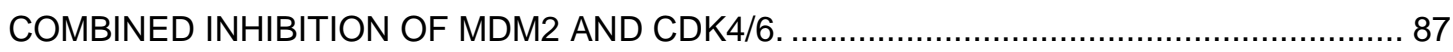

FIGURE 3.3 S 4 ALTERNATE RNA POLYMERASE II ANTIBODIES INDICATE DECREASED

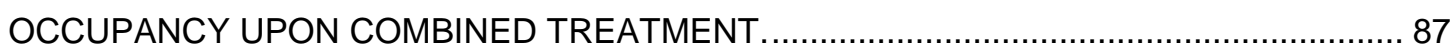

FIGURE 41 SCHEME EMPLOYED FOR FORTIFYING P53 ACTIVITY AND THE RESULTING

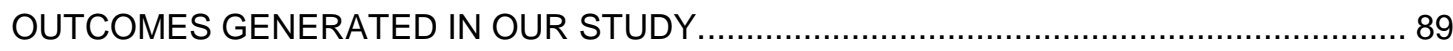

FIGURE 5 A 1 ALTERNATE CELL LINES ALSO SHOW DIMINISHED ACTIVATION OF P53 WHEN COMBINED WITH CDK4/6 INHIBITOR.

FIGURE 5 A 2 PHOSPHORYLATION OF P53 AT S20 AND S46 UPON P53 ACCUMULATION

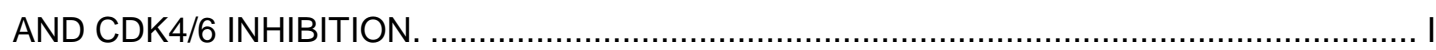

FIGURE 5 A 3 TREATMENT WITH PROTEASOME INHIBITOR PARTLY RESCUED MDM2

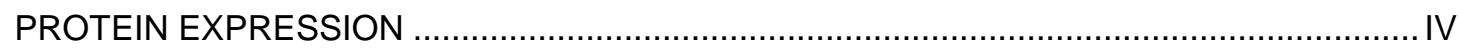

FIGURE 5 A 4 COMBINATION OF A MDM2 ANTAGONIST WITH CDK4/6 INHIBITOR DOES NOT LARGELY AFFECT PS2 AND PS5 RNA POLYMERASE II AT P53 TARGET GENES......... VII

FIGURE 5 A 5 CDK4 AND MDM2 INHIBITION LEADS TO SLIGHTLY ELEVATED ENRICHMENT OF CYCLIN D1 AT THE PROMOTERS OF P53 TARGET GENES. 


\section{LIST OF TABLES}

TABLE 1 LIST OF PRIMERS USED FOR GENE EXPRESSION STUDIES............................... 78

TABLE 2 LIST OF PRIMERS USED FOR CHROMATIN IMMUNOPRECIPITATION 81 


\section{ABSTRACT}

p53 is one of the most extensively studied tumor suppressor proteins, and its gene is mutated in $50 \%$ of human cancers. Stress signals activate p53 and its transcriptional machinery which lead to cellcycle arrest, apoptosis or senescence. MDM2, its target gene product and the main negative regulator can bind to $\mathrm{p53}$, and mark it for proteasomal degradation via its RING finger domain and the associated E3 ubiquitin ligase activity. Preventing the p53-MDM2 interaction via small molecule inhibitors appears to be the most obvious strategy to eliminate tumors harboring wild-type p53. However, despite extensive research, clinical responses to these drugs have been disappointing. Patient studies do not indicate durable tumor regression upon administration of these drugs but rendered adverse effects like nausea, vomiting, diarrhea and hematological disorders such as neutropenia and thrombocytopenia.

In this thesis, we have aimed to evaluate MDM2 inhibitors in combination with other drugs that lead to either synergism or antagonism with respect to p53 response in three diverse manners.

Firstly, we have utilized the concept of cyclotherapy and MDM2 inhibition to protect normal cells against agents like WEE1 inhibitors that causes replicative stress. We have found that pre-treatment of cells with MDM2 inhibitors shielded them from cytotoxic effects of WEE1 inhibition. This strategy would be beneficial in the clinics to target p53-deficient cancer cells, while protecting the p53-wildtype normal cells. Secondly, we targeted the two negative regulators of p53, namely MDM2 and WIP1 by inhibitors to enhance cellular cytotoxicity. This led to an increased expression and occupancy of p53 at its target genes and also caused a senescent phenotype. Finally, we observed an unexpected antagonism between inhibitors of MDM2 and CDK4/6 regarding cell viability and p53 response, indicating CDK4 is a supportive kinase for p53 activity.

Thus, our studies indicate three different approaches to modulate the cytotoxic effects of MDM2 inhibition. Taken together from our observations, we conclude that MDM2 inhibition might lead to patient benefit by changing the purpose of the drug; by combining it with other drugs that can mediate stronger toxicity; and lastly by targeting the correct population of tumors to obtain robust effects. 


\section{INTRODUCTION}

\subsection{The family of TP53 - "Protector of our genome"}

In response to diverse stress stimuli and DNA damage, the tumor suppressor protein p53 executes various roles including cell-cycle arrest, senescence and apoptosis, thereby making it one of the most widely studied proteins in cancer biology. Originally identified as a $54 \mathrm{kDa}$ protein bound to SV40 large-T antigen [1-3], the TP53 gene is frequently mutated $(\sim 50 \%)$ in human tumors $[4,5]$ rendering it non-functional.

In cancers, p53 is often inactivated by viral proteins or by upregulation/ amplification of its negative regulators. Studies have also indicated germline mutations in the p53 gene resulting in a rare inherited autosomal dominant disorder known as Li-Fraumeni syndrome [6, 7]. Such patients are characterized by their high risk towards the development of sarcomas, adrenocortical carcinoma (ACC), breast cancer, leukemia, and brain tumors [8, 9]. Research conducted in mice deficient for p53 displayed normal embryonic development but were predisposed to spontaneous tumors including lymphoma and sarcoma; thereby confirming its tumor suppressive functions [10].

p63 and p73 are two homologues of p53 [11, 12] with overlapping yet distinct functions. Like p53, p73 can be activated upon DNA damage that can lead to the elimination of cells via apoptosis [13]. p73 was recently described to modulate airway multiciliogenesis in mice by affecting the master regulator of ciliogenesis, namely FoxJ1 [14]. Mice deficient for TP73 showed defects in fluid dynamics in the central nervous system, neurogenesis, reproductive and social behavior [15]. Moreover, p73 was shown to be essential for maintaining genome stability when p53 was inactivated [16]. On the other hand, p63 plays a major role in squamous epithelial development as observed in TP63 knockout mice. These mice exhibited defects in epithelial tissues such as absence of hair, skin and sweat glands along with faulty limb and craniofacial development $[17,18]$.

\subsection{Transcriptional role of p53}

p53 functions as a homotetramer and recognizes its responsive element on the DNA that consists of two repeats of the consensus sequence $5^{\prime}-\operatorname{PuPuPuC}(\mathrm{A} / \mathrm{T})(\mathrm{T} / \mathrm{A}) \mathrm{GPyPyPy}-\mathrm{3}^{\prime}[19,20]$. However, on the PIG3 promoter $p 53$ identifies a pentanucleotide microsatellite sequence (TGYCC) $n$ (where $n=15$ ) [21]. It acts as a transcription factor by binding to these responsive elements and augments the rate of transcription [22]. A plethora of genes have been identified to be directly regulated by p53. Few of the best understood genes include CDKN1A or p21 [19] that is responsible for cell-cycle arrest; PUMA, NOXA, BAX and others that induce apoptosis [23]; and Plasminogen Activator Inhibitor -1 (PAl-1) [24] whose expression has been linked to mediate senescence. p21 has also been described to cause senescence via cell-cycle arrest by suppressing the factors that promote cellular proliferation [25]. 


\subsection{Structure of $\mathrm{p} 53$}

The p53 protein consists of 393 amino acids which undergoes numerous post-translational modifications including phosphorylation, acetylation, ubiquitination, sumoylation, and neddylation (Figure 2 1) [26].

p53 comprises of two Iransactivation Domains (TAD1 and TAD2) that are intrinsically disordered i.e. it lacks a fixed three-dimensional structure $[27,28]$. These domains can bind to MDM2, a target gene of p53 that is also its negative regulator, and p300/ㅅAP Responsive Element Binding- Protein (CREB) Binding Protein (CBP), a histone acetyltransferase which is a general transcriptional coactivator [29].

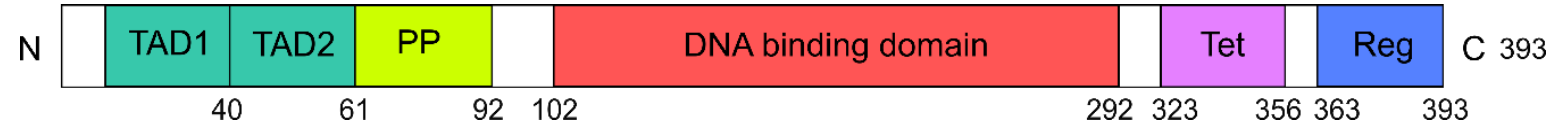

Figure 21 Schematic structure of p53.

p53 consists of two transactivation domains (TADs), a proline-rich domain (PP), a central DNA binding domain (DBD), a tetramerization domain (Tet) and a C-terminal regulatory domain (Reg). Adapted from [30].

The N-terminus of the p53 protein is subjected to extensive post-translational modifications [31] in response to stress cues that lead to its activation and stabilization. The TAD2 region present at the $\mathrm{N}$-terminus also interacts with the p62 subunit of Iranscription Factor IIH (TFIIH) complex within the general transcriptional machinery, and this interaction is pivotal for carrying out its role as a transcription factor [32]. The Proline rich region spanning amino acid residues 61-92 contains five repeats of the sequence PXXP. This region plays a role in p53-mediated induction of apoptosis [3335]. The DNA binding domain of $p 53$ is responsible for its binding to the responsive elements present on DNA that enables the transcription of its target genes. Most of the hotspot mutations occur within this region [36]. These mutations prevent sequence-specific binding of p53 to the promoter of its target genes. $10 \%$ of these hotspot mutations arise due to the lack of a functional protein by frameshift or nonsense mutations whereas the remainder is a result of missense mutations. The tetramerization domain enables the formation of an active p53 tetramer from two homodimers [37] that enables the correct conformation for binding to DNA. It also contains a Nuclear Export Signal (NES) between residues 340 and 351 [38] which allows the export of p53 into the cytoplasm. Additionally, the p53 $\underline{\text {-T}}$-Terminal Domain (CTD) undergoes extensive post-translational modifications that are important for the activity and stability of the protein. Of importance, MDM2-mediated ubiquitination leading to the degradation of p53 protein occurs within this terminal region, owing to the numerous lysine residues that are present [39-41].

\subsection{Cellular functions of p53- "Get, set, and action"}

To maintain homeostasis in normal unstressed cycling cells, p53 is kept at low levels owing to its short half-life ( 5-20 mins) (Figure 22 ). Additionally, these low levels are kept intact by the negative regulators of p53 i.e. E3 ubiquitin ligases such as MDM2/MDMX [42], P $53-\underline{A}$ ssociated $\underline{\text { Cellular }}$

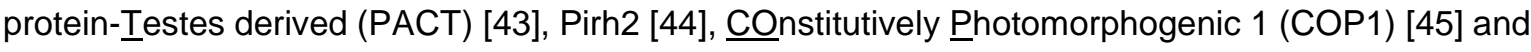


phosphatases such as Wild-type $\underline{P} 53$-induced phosphatase 1 (also referred to as WIP1/PPM1D) [46, 47].

Upon exposure to various genotoxic insults including DNA damaging agents, hypoxia and nucleotide deprivation, p53 is stabilized and activated which leads to a steady increase in its levels (Figure 22 ). This is achieved by post-translational modifications on the $\mathrm{N}$ - and $\mathrm{C}$-terminal regions that increase the affinity of p53 to its target genes and enable its transactivation which helps to promote either the elimination of the cell or support its repair, thereby preventing the risk of transmitting mutations to the next generation.

Basal TP53

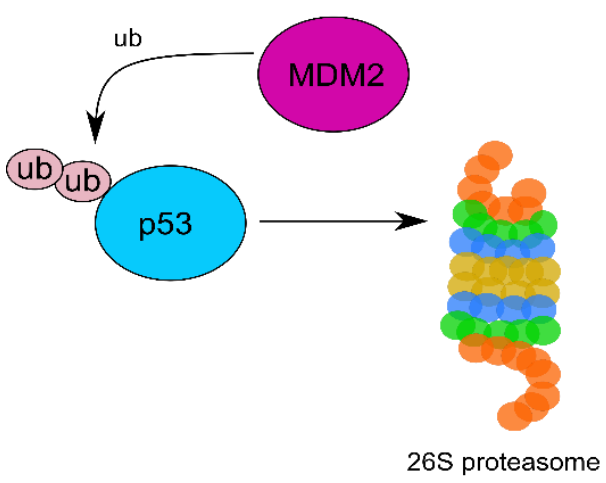

Activated TP53

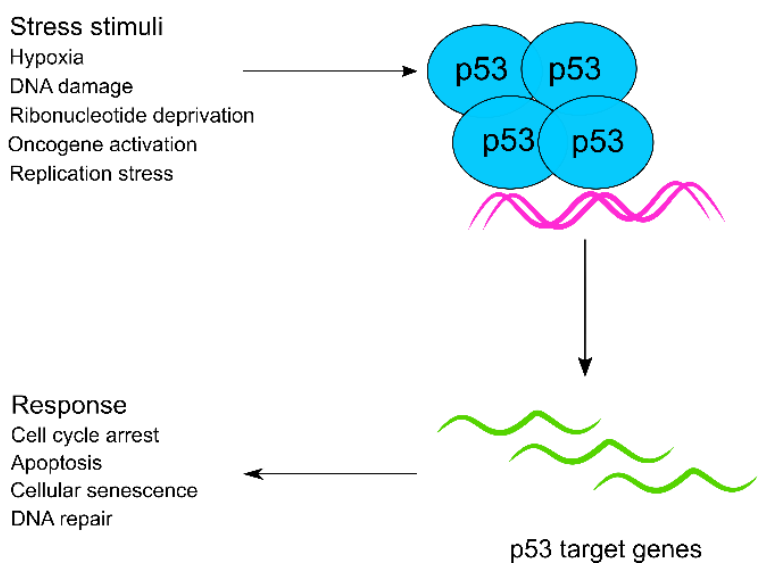

Figure 22 Activation of p53

Homeostasis of p53 levels are maintained in normal cells by MDM2. Upon ubiquitination of p53 by MDM2, p53 undergoes proteasomal degradation via the $26 \mathrm{~S}$ proteasomal machinery. In response to external stress, p53 accumulates within the cell and forms a tetramer; binds to its target genes at the responsive elements and transcribes the various genes involved in cell-cycle arrest, apoptosis, senescence, and repair. Adapted from [48].

The resulting fate of the cell is largely context-dependent; whether the cell undergoes cell-cycle arrest, permanent cell-cycle arrest (i.e. senescence) or apoptosis. Minor DNA damage could cause activation of cell-cycle arrest or DNA repair mechanisms while strong p53 activating signals might result in senescence or apoptosis. A large amount of work has been carried out to understand the resulting cell fate upon different stress stimuli, at varying time points with diverse cell lines using Chromatin immunoprecipitation sequencing (ChIP-seq), Global-ㅡun on sequencing (GRO-Seq) and RNA-sequencing (RNA-seq) studies painting a more complex picture of p53 gene regulation [49, 50]. An overview of the important roles of p53 and the genes responsible for them are outlined below.

\subsubsection{Cell-cycle arrest}

Transient arrest of cells at the G1-S interphase ensures detection and repair of damaged DNA before initiation of replication in S-phase. The G2-M transition in the cell-cycle is another checkpoint where cells are checked for unreplicated or damaged DNA after S-phase before they undergo mitosis (Figure 23 ). If left unrepaired, faulty or damaged DNA entering M-phase could lead to mitotic catastrophe, a form of cell death that occurs due to aneuploidy. 


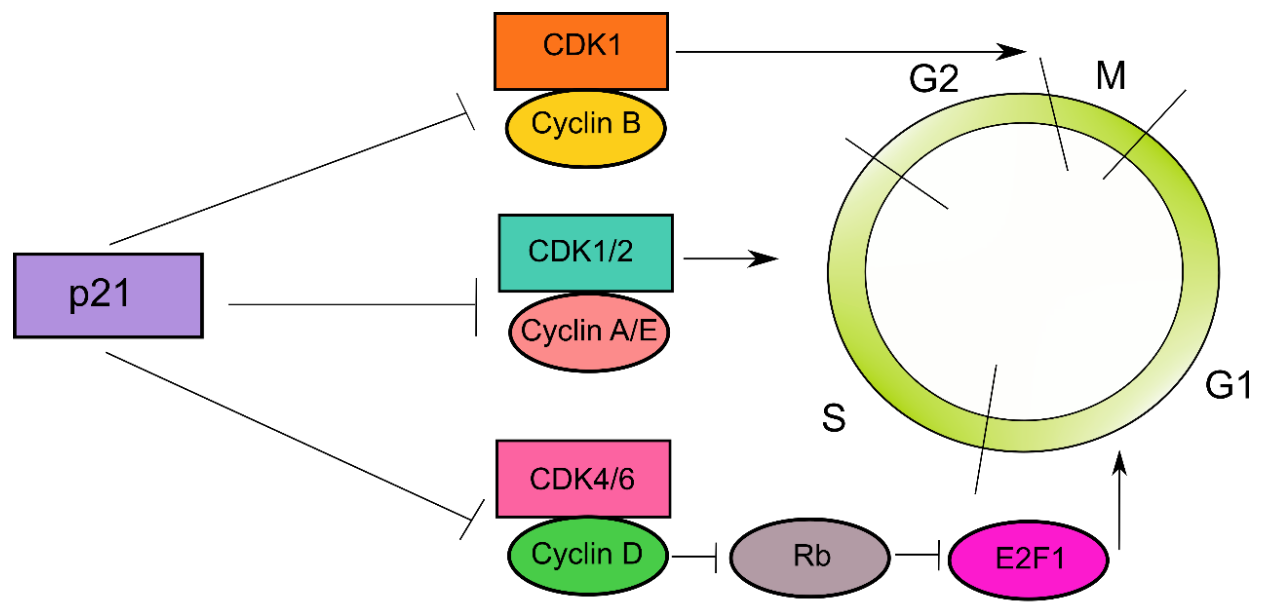

Figure 23 p21-mediated cell-cycle arrest.

Upon activation of p53, p21; a CDK inhibitor, which is one of the primary target genes of p53 is transcribed which arrests the cells at the G1-S phase via CDK4/ Cyclin D, Rb and E2F1 proteins; S- phase by CDK1/2 and Cyclin A/E complexes and G2-M phase by CDK1/ Cyclin B complexes. Adapted from [51]

CDKN1A is the primary target gene of p53 which mediates cell-cycle arrest. It is a cell-cycle dependent kinase (CDK) inhibitor which inhibits the kinase activity of CDK4/6, CDK1 and CDK2, thereby preventing the cells from progressing at G1-S, S and G2-M phases of the cell-cycle respectively. Although mice lacking CDKN1A were not prone to spontaneous tumors [52], they showed defective G1 checkpoint activity upon DNA damage and p53 activation [53, 54].

Other p53 targets that can cause cell-cycle arrest include calveolin-1 at the G0-G1 transition [55], and BTG anti-proliferation factor 2 (BTG2) at the G1-S transition [56]. Likewise $\underline{\text { Growth- }}$ Arrest and

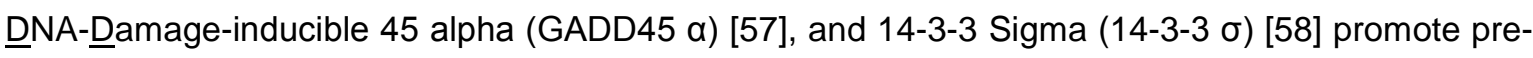
mitotic cell-cycle arrest upon DNA damage via p53 accumulation.

\subsubsection{DNA repair}

Initiating cell-cycle arrest by p53 activation at G1-S transition allows for efficient repair of damaged DNA prior to synthesis. p53 activates various repair pathways like Nucleotide Exxision Repair (NER) upon DNA damage by activating DNA Damage-B̈inding protein 2 (DDB2) [59] and Xeroderma

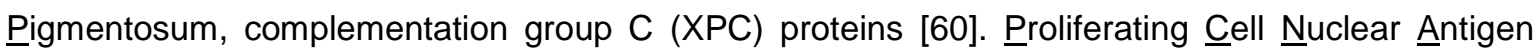
(PCNA), a co-factor that helps to recruit key players to the replication fork [61] is a p53-responsive target [62] which also assists in DNA repair by activating DNA damage tolerance pathways including Iranslesion DNA Synthesis (TLS). Ubiquitination of PCNA helps to recruit repair proteins like RAD6RAD18 that initiates DNA repair mechanisms [63]. p53 can also initiate translesion DNA synthesis in cells exposed to low doses of UV irradiation by activating Pol eta (POLH) $[64,65]$. This activates the ATM/CHK2 pathway and allows the cells to bypass the damage and helps in efficient restart of the damaged fork. 


\subsubsection{Apoptosis}

In cases of overwhelming stress, if the DNA damage is not sufficiently repaired by the various mechanisms as outlined in section 2.4.2, the cells activate a programmed cascade of events that lead to apoptosis. This can be triggered by two types of stimuli, namely extrinsic and intrinsic. Extrinsic stimuli encompasses death ligands such as the Fas ligand (Fas-L) or the Iumor-Necrosis Factor- $\alpha$ (TNF- $\alpha$ ) that bind to cell-surface receptors [66]. On the other hand, intrinsic stimuli center around the mitochondria upon DNA damage, UV irradiation, stress or oncogene activation that is regulated by the pro-apoptotic and anti-apoptotic members of the $\underline{B}$ - ell Lymphoma $2(\mathrm{Bcl}-2)$ family [67]. Cysteine aspartic proteases (caspases) which have intrinsic cysteine protease activity help in cleaving their target proteins at aspartate residues. These enzymes play a pivotal role in apoptosis by cleaving nuclear lamins, $\underline{P}$ oly- $\underline{A} P$ P Ribose Polymerase (PARP), and Inhibitor of the $\underline{\text { Caspase- }}$ activated DNAse (ICAD). This proteolytic cleavage frees the DNAse- CAD leading to DNA fragmentation and nuclear blebbing, consequently resulting in apoptosis.

p53 can activate genes of both the extrinsic and the intrinsic apoptotic pathway. FAS, Iumor Necrosis Factor S6 (TNFS6) [68-70] and Iumor necrosis factor-Related Apoptosis-Inducing Ligand (TRAIL) $[71,72]$ are some of the genes activated upon p53 accumulation that cause caspase-dependent apoptosis via procaspase 8 . On the other hand, p53 also activates pro-apoptotic Bcl-2 members such

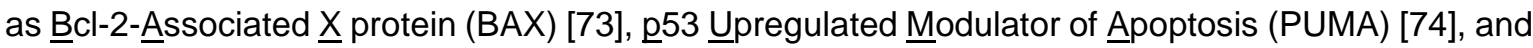
NOXA [75] that control the release of cytochrome $c$ from the mitochondria. The released cytochrome c binds to another p53 target, Apoptosis- $\underline{\text { Peptidase }}$ Activating Factor-1 (APAF-1) [76, 77] and procaspase 9 to form the apoptosome. This triggers the cascade of effector caspases such as caspase-3 and caspase-7, ultimately leading to apoptosis.

\subsubsection{Senescence}

A state of cell-cycle arrest where the cells retain their metabolic activity but cease to divide is termed as senescence. Induction of senescence is attributed to the suppression of malignant lesions. It is initiated by DNA- Double-Strand Breaks (DSBs), or acute/chronic stress within the cell. p53 activates p21, Promyelocytic Leukemia protein (PML) [78], Plasminogen Activator Inhibitor-1 (PAI1) [24] and E2F7 [79] that initiates an oncogene-induced senescence program. Apart from p53 and Rb [80-82], oncogene-induced senescence can also be mediated by kinases such as p38, and PI3K/AKT/mTOR pathways [83, 84]. By suppressing cellular proliferation, senescence acts as a barrier to the transformation of cells.

\subsection{Post-translational modifications on p53}

p53 undergoes numerous Post-Iranslational Modifications (PTM) on its $\mathrm{N}$ and C-terminal domains (Figure 24 ). In response to various stress stimuli, p53 can be phosphorylated by numerous DNA Damage $\underline{R}$ esponse (DDR) kinases like Ataxia Ielangiectasia Mutated (ATM) [85-87], Ataxia Telangiectasia and Rad-3 related (ATR) [88], DNA-dependent Protein Kinase (DNA-PK) [89, 90],

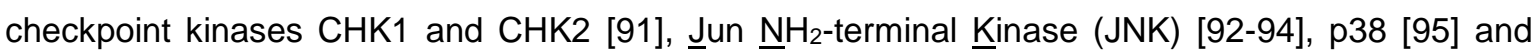
others. 
Particularly, in response to DNA double-strand breaks, p53 is phosphorylated at Ser15 (Ser18 in mouse) by ATM. ATM/ATR kinases can also mediate the phosphorylation of p53 at Ser6, Ser9, Ser20, Ser46 and Thr18 [96, 97].

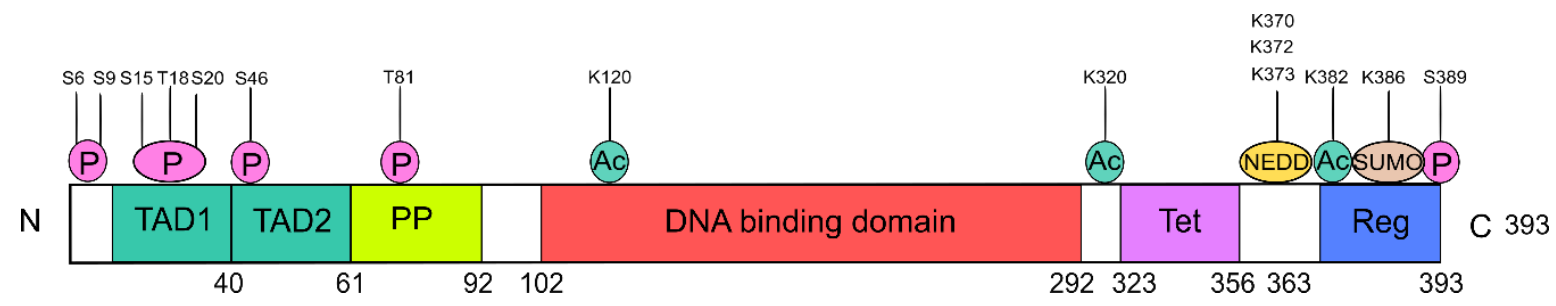

Figure 24 Post-translational modifications on the p53 protein.

Important post-translational modifications on $\mathrm{p} 53$ are indicated at the respective domains. P signifies phosphorylation, Ac depicts acetylation, SUMO denotes Sumoylation, and NEDD indicates neddylation. Adapted from [31].

Upon exposure to UV irradiation, p53 is phosphorylated at Thr81 by JNK [98] and Ser389 by p38 kinase [99]. Homeodomain Interacting Protein Kinase 2 (HIPK2) can mediate the phosphorylation of p53 on Ser46 upon UV irradiation [100, 101] which has been implicated to modulate the cell fate towards apoptosis. All the above modifications are responsible for increasing the stability and activity of p53 as a transcription factor.

Acetylation of lysine residues at the C-terminal region of p53 exposes the DNA binding domain leading to enhanced transcription of its targets [102]. This is carried out by the p300/CBP/PCAF family of Histone Acetyltransferases (HATs). Apart from the C-terminus, the DNA binding domain of p53 can also be acetylated at Lys120 upon DNA damage by Tip60. This is critical for mediating p53dependent apoptosis via BAX and PUMA [103, 104].

Neddylation of p53 can occur at Lys370, Lys372, Lys373 by MDM2 and Lys320, Lys321 by F-ㅁo protein 11 (Fbx11). The above modifications lead to the inhibition of p53-mediated transcription [105, 106]. Sumoylation, another post-translational modification carried out by an ubiquitin-like protein named SUMO-1, occurs at Lys386 upon UV irradiation which enhances the activation of p53 target genes [107]. The exact outcome of these modifications remains largely elusive.

While p53 is subject to various modifications that activate the protein, these post-translational modifications are also reversed by various proteins that attenuate this signaling axis, thereby negatively regulating p53 activity. Members of the Protein phosphatase $2 \mathrm{C}$ (PP2C) family such as Wild-type p53 Induced Phosphatase (WIP1 or PPM1D) are activated upon DNA damage, which can dephosphorylate p53, thereby leading to its downregulation $[108,109]$. Thus, they act as negative regulators of $p 53$ activity.

Histone Deacetylases (HDACs) including HDAC1 and Sirtuin1 (SIRT1) can remove acetyl groups from p53, causing decreased transcriptional activity and cell survival upon stress stimuli $[110,111]$. 
In 2004, it was discovered that Set9, a histone lysine methyltransferase methylates p53 at Lys372 which correlated with increased stability of p53 [112].

Finally, p53 undergoes ubiquitination which is highly reversible and dynamic. It can undergo monoubiquitination or polyubiquitination, each leading to a distinct outcome. MDM2, an E3 ubiquitin ligase and a transcriptional target of p53 ubiquitinates it at Lys370, Lys372, Lys373, Lys381, Lys382 and Lys386 [113]. This contributes to the nuclear export of p53 [114]. Increased expression of MDM2 that is induced upon p53 activation provides a pathway for polyubiquitinating p53, thereby leading to its degradation. Thus, MDM2 induction by p53 forms a negative feedback loop that keeps p53 levels in check after the damage has been repaired [40, 115]. Independent of MDM2, the ubiquitin ligase Pirh2 functions as a negative regulator of p53 by binding to $p 53$ and inhibiting its growth suppressive functions by ubiquitination and proteasomal degradation [44]. Furthermore, COP1 was found to be a p53-inducible gene, and its product binds to p53 and inhibits p53-dependent transcription and apoptosis in an ubiquitin-dependent manner [45]. ARF-BP1 directly binds and ubiquitinates p53 in p53 wild-type cells and its inactivation is essential for p53 activation [116].

In contrast to the ubiquitinating enzymes, deubiquitinating enzymes (DUBs) are proteases that cleave ubiquitin-linked molecules on the last residue of ubiquitin (Gly76) after the terminal carbonyl. Specific Ubiquitin Specific Protease (HAUSP or USP7) was shown to strongly stabilize p53 even in the presence of MDM2 to induce cell-cycle arrest and apoptosis [117].

Thus, p53 is regulated by diverse post translational modifications which lead to the activation or repression of its target genes in response to stress signals. In the next section, the focus will be on the target gene of $\mathrm{p} 53$ and its negative regulator - MDM2.

\section{6 "Self-controlling p53"- MDM2}

MDM2 (uuine double-minute 2) was originally identified as one of three genes which was overexpressed in a spontaneously transformed murine cell line (3T3 fibroblasts; [118]) and its product was found to bind to and downregulate p53 [119, 120]. Mice deficient for MDM2 were embryonically lethal, however when p53 was co-depleted, they were viable and displayed normal development [121, 122]. As stated earlier, MDM2 is a target gene of $p 53$ whose product is an E3 ubiquitin ligase. The ubiquitin ligase activity of MDM2 is carried out by its conserved $\mathrm{COOH}$ terminal RING finger domain which can ubiquitinate $p 53$ at its $\mathrm{C}$-terminus. This causes the degradation of p53 via the $26 \mathrm{~S}$ proteasome. MDM2 is an oncogene that is frequently overexpressed in sarcomas, leukemia and lymphoma which are associated with poor prognosis [120, 123].

\subsection{Structure of MDM2}

The MDM2 protein consists of 491 amino acid residues and interacts with the transactivation domain of p53 via its $\mathrm{N}$-terminus (Figure 25) [124].

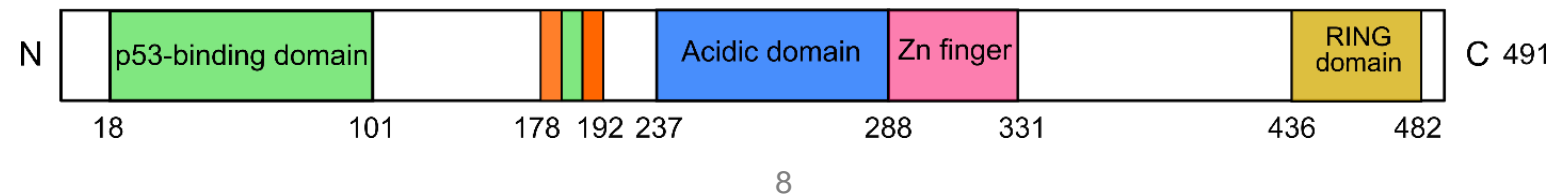




\section{Figure 25 Domain structure of MDM2.}

The different domains of MDM2 protein are indicated. The acidic domain and Zn finger domain are flanked by the p53-binding domain at the N-terminus and the Really Interesting New Gene (RING) finger domain at its Cterminus. Adapted from [125].

The central acidic domain has been suggested to interact with the Retinoblastoma $(\mathrm{Rb})$ protein at its C-pocket [126]. Binding of MDM2 with Rb prevents the E2F-Rb interaction, thereby allowing cells to progress through the G1-S phase of the cell-cycle. Moreover p19ARF (Alternate Reading Frame), a member of the INK4a gene locus was found to physically interact with MDM2, thereby preventing MDM2-mediated p53 degradation [127-129]. This can occur by sequestering MDM2 to the nucleolus along with ARF that leads to the activation of p53 in the nucleoplasm or by binding to MDM2 and causing its degradation. Other binding partners of MDM2 at this domain include the co-activator p300 [130], and RPL11 which sequesters MDM2 in the nucleolus resulting in p53 stabilization [131]. Mutations in the zinc finger domain of MDM2 disrupts its interaction with ribosomal proteins L5, L11 and L23 inhibiting its ubiquitin ligase activity and thereby its ability to degrade p53 [132].

The two important domains in MDM2 protein include the $\mathrm{N}$-terminus p53 binding domain and the $\mathrm{C}$ terminus RING finger domain. Crystallographic data as well as biochemical experiments have indicated that the N-terminus of MDM2 (25-109aa) forms a deep hydrophobic cleft into which the Nterminal TAD (15aa amphipathic residue) of p53 binds [39, 124, 133]. Residues Phe19, Trp23, and Leu26 on p53 are most critical for binding to MDM2 [133, 134] while Gly58, Gly68, Val75, or Cys77 residues on MDM2 are critical for binding to p53 [135]. The Thr18 residue on p53 was identified to be important for the stability of the p53 $\alpha$-helix [124]. Phosphorylation of this residue abrogates p53MDM2 binding by nearly 10 -fold while no effect on binding was detected if p53 was phosphorylated at Ser15 and Ser20 residues [136].

MDM2 can function as an E3 ubiquitin ligase towards itself and p53 [41, 137, 138]. Monoubiquitination of p53 is mediated by MDM2 while polyubiquitination requires the ubiquitin ligase activity of p300 [139, 140]. Mutations in the RING finger domain (C464A) abolished the E3 ubiquitin ligase activity but not its interaction with p53 [141].

Oren and colleagues also reported that MDM2 can be acetylated at its RING domain by p300 that accentuates the transcriptional activity of p53 due to MDM2 degradation [142].

Apart from this, MDM2 also contains a nuclear localization sequence (residues 181-185) and a nuclear export signal (residues 190-200). This helps in shuttling MDM2 in and out of the nucleus [38, 143].

MDMX, an MDM2 homologue can heterodimerize with MDM2 via their RING finger domains. However, unlike MDM2, MDMX lacks the intrinsic E3 ubiquitin ligase activity [144, 145]. Despite that, MDMX can bind to p53 through its p53-binding domain, which masks the TAD of p53 that leads to the attenuation of the transcriptional activity of p53 in a manner similar to MDM2 $[146,147]$. 


\subsection{Transcriptional regulation of MDM2}

The transcription of MDM2 is regulated by two distinct promoters - P1 and P2 [148] (Figure 26 ). The two isoforms migrate at $90 \mathrm{kDa}$ and $75 \mathrm{kDa}$ respectively due to initiation of translation from two distinct AUG start codons. The P1 promoter is situated upstream of exon one and controls the basal expression of MDM2, while the P2 promoter that is present within the first intron is highly regulated and inducible [149]. The p53 responsive elements are present upstream of the P2 promoter; thereby enabling p53-induced expression of MDM2 [150].
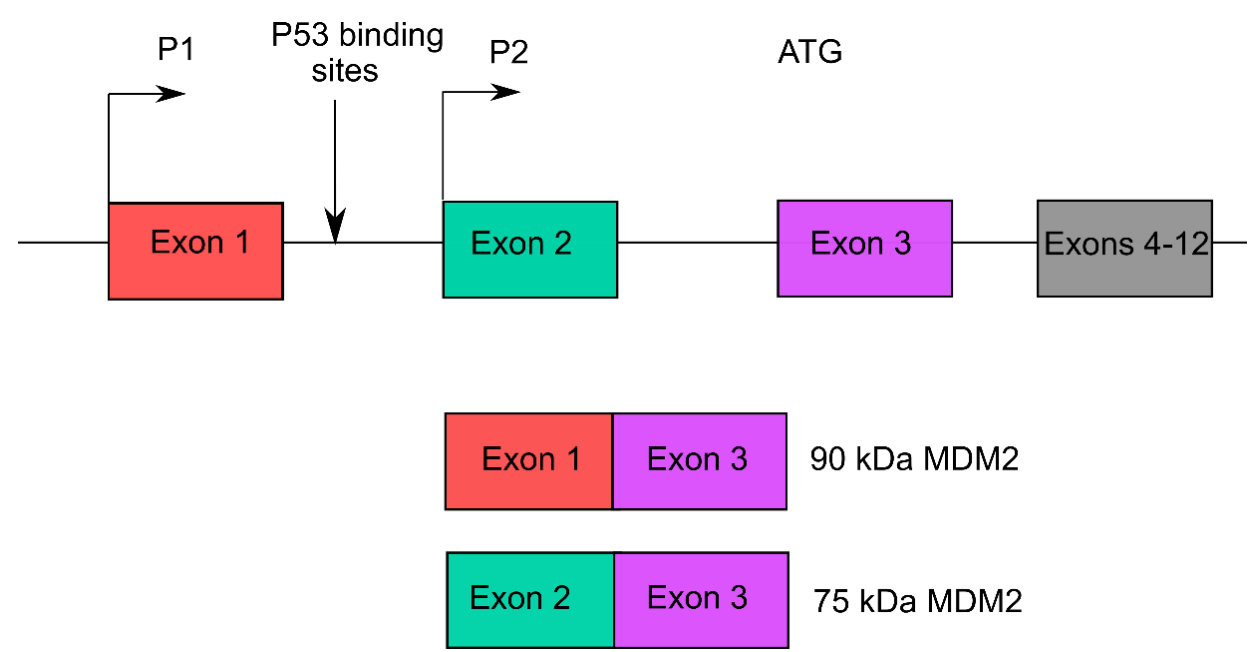

Figure 26 P1 and P2 promoters of MDM2.

Schematic representation of the 5' end of the MDM2 gene. The long isoform of the MDM2 protein includes exon 1 and 3 while the short isoform; P2 includes the p53 binding sites located upstream of exon 2. Adapted from [151].

\subsection{Post-translational regulation of MDM2}

MDM2 undergoes phosphorylation at its $\mathrm{N}$-terminus p53-binding domain, central acidic domain and at the C-terminus RING finger domain (Figure 27 ).

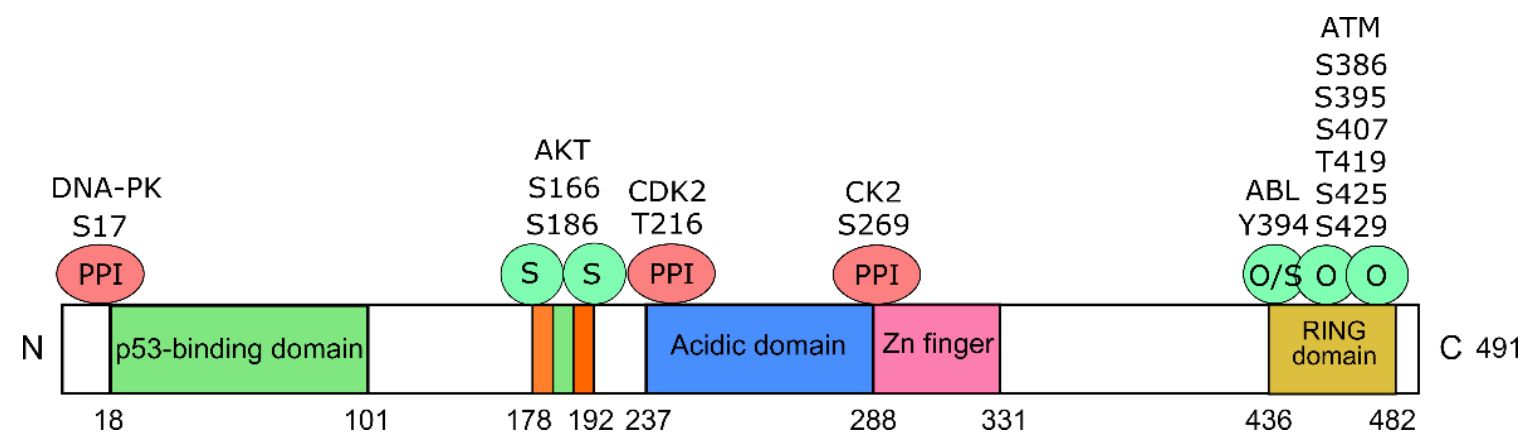

Figure 27 Phosphorylation sites on MDM2.

Green circles indicate sites that increase MDM2/MDMX mediated inhibition of p53; orange circles depict inhibition of MDM2/MDMX dependent inhibition of p53. PPI indicates phosphorylations on MDM2 that affect p53 interaction; $S$ indicates sites that modulate the stability of MDM2 upon phosphorylation, $O$ refers to phosphorylations that change the oligomerization of the protein and $\mathrm{O} / \mathrm{S}$ refers to changes in oligomerization and stability. Drawn from [152]. 
In response to DNA double strand breaks, ATM can phosphorylate MDM2 at Ser395 which impairs its ubiquitin ligase activity on p53 and activates p53 [153]. On the other hand, WIP1 phosphatase maintains this balance by dephosphorylating MDM2 after the stimulus is removed [154]. Other kinases that can phosphorylate MDM2 include AKT which phosphorylates MDM2 at Ser166 and Ser186 leading to its stabilization and the subsequent inhibition of p53 [155]. Phosphorylation by DNA-PK at Ser17 of the MDM2 protein causes the dissociation of p53 from its negative regulators, thereby allowing p53 stabilization [156]. S6K1 kinase, a downstream factor of mammalian target of rapamycin (mTOR) pathway mediates phosphorylation of MDM2 at Ser163 in response to doxorubicin that hampers the ubiquitination of p53 by MDM2 [157]. Cyclin-dependent kinases - CDK1 and CDK2 phosphorylates p53 at Thr216 that might affect MDM2 binding to other proteins [158]. CAbl can block ubiquitination and proteasomal degradation of $p 53$ by phosphorylating MDM2 at Tyr394 [159].

P14-ARF has been shown to enhance sumoylation of MDM2 (residues 82-101) by blocking its ubiquitination activity [160] and thereby promoting p53 response. In addition to its role in maintaining p53 levels, p53-independent functions of MDM2 have been reported. Few of the important ones are listed below.

\subsection{0 p53-independent roles of MDM2}

Apart from being a negative regulator of $\mathrm{p} 53, \mathrm{MDM} 2$ has been described to have $\mathrm{p} 53$-independent roles. In vivo studies have shed light into the p53-independent role of MDM2 during tumorigenesis. Mice lacking MDM2 were found to be embryonically lethal while this phenotype could be reversed if p53 was co-depleted [121]. In the absence of p53, association of MDM2 with NBS1 of the $\underline{M}$ RE11/ㄹAD50/NBS1 (M/R/N) DNA repair complex resulted in the inhibition of DNA double-strand break repair; indicating a role of MDM2 in maintaining genomic stability [161]. Studies by Mulay and co-workers showed that MDM2 was essential for Toll-like receptor (TLR)-mediated cytokine production; implicating its role in inflammation by enhanced Nuclear Factor 'kappa-light-chainenhancer' of activated B-cells (NF-KB) signaling [162]. Recent findings have also identified roles for chromatin-bound MDM2 in the transcriptional control of genes involved in amino acid metabolism and redox homeostasis [163] a well as lineage-specific genes by its interaction with the Polycomb repressive complexes (PRC) 1 and 2 [164].

These p53-dependent and independent roles of MDM2 have made it an interesting drug candidate in cancer therapy. By targeting the MDM2-p53 interaction, we can activate the transcriptional network of p53 which can be used efficiently to treat p53 Wild-type (WT) tumors. The next section will provide a short summary.

\subsection{Targeting the oncogene - MDM2}

By interfering with the protein-protein interaction of MDM2 and p53 using peptides or other small molecules, treatment of cancers with wild-type p53 can be achieved (Figure 28 ). 


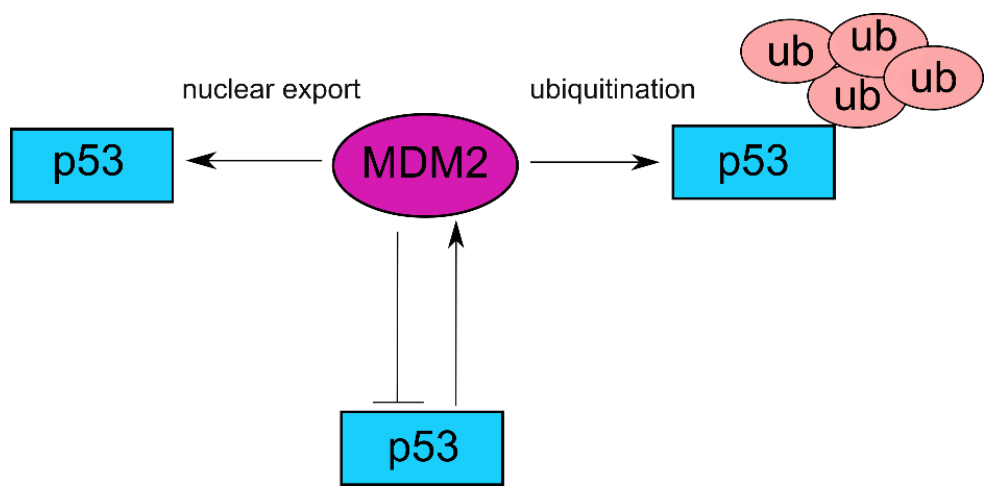

Figure 28 Negative feedback loop of MDM2 and p53.

Upon activation, p53 transcribes its target genes. One of its main targets, MDM2 accumulates within the cell which also acts as its negative regulator and marks p53 for monoubiquitination that promotes nuclear export or polyubiquitination followed by proteasomal degradation. Adapted from [165].

Although MDM2 and p53 have been studied extensively for over three decades, there is currently no Food and Drug Administration (FDA) approved drug for MDM2 inhibitors. The first small, potent and specific inhibitor of MDM2 came in 2004 from the company Hoffmann-La Roche where Nutlin-3a, one amongst many Nutlins could bind to MDM2 in vitro at an Inhibitory Concentration (IC) of 90nM. Nutlin could inhibit the interaction of MDM2 with p53, and activate the p53 transcriptional network, thereby displaying selectivity towards p53 wild-type cancer cell lines [166] (Figure 29 ).

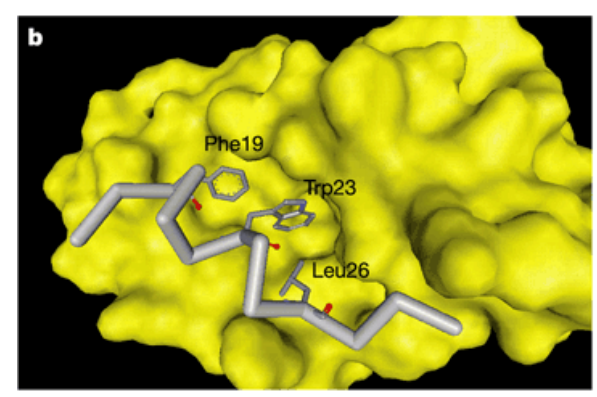

Figure 29 Targeting the MDM2-p53 interaction.

MDM2 protein is indicated in yellow which binds to the TAD1 of p53. p53 (residues 17-29) backbone is indicated in grey with the side chains Phe19, Trp23 and Leu26 that are essential for the interaction to occur [48]. These inhibitors bind competitively to this pocket.

After further optimization, another MDM2-p53 inhibitor RG7112 (RO5045337) entered clinical trials for liposarcoma patients. Although there was a clear activation of p53 indicated by increased p21 levels and signs of apoptosis in the tumors, only a partial response was observed in patients. Moreover, patients suffered from severe adverse effects including neutropenia, thrombocytopenia, diarrhea, vomiting and nausea [167]. In addition, two out of six other MDM2 inhibitors that entered clinical trials have initial reports available which include RG7388 also known as Idasanutlin [168] and Ml-77301 [165]. However, two challenges that these drugs faced in clinical trials were dose- 
dependent toxicity that led to hematological disorders and detection of p53 mutations, especially at the DNA binding domain of p53 in tumors that were initially wild-type.

Other inhibitors of MDM2 that are being tested in vitro and in vivo affect its ubiquitin ligase activity at the RING finger domain and its interaction with MDMX. They include MEL23/24 [169], HLI98 [170], and RO-5963 [171]. All these drugs indicated a strong inhibition of the interaction between MDM2MDMX resulting in the stabilization of p53 and subsequent induction of p53-dependent apoptosis.

p53- mimetic stapled peptides have been a topic of intense research. ATSP-7041 showed on-target mechanism of action by inhibiting the MDM2-MDMX interaction and activation of $p 53$. This led to tumor growth suppression in an MDM2/MDMX overexpression xenograft model with favorable pharmacokinetic properties [172].

APR-246 (PRIMA-1MET), a p53-mutant re-activator has shown promising results in refractory hematological malignancies and prostate cancer by restoring the transcriptional activity of unfolded wild-type or mutant p53 [173]. Induction of apoptosis was observed upon its combination with cisplatin and fludarabine $[174,175]$ in p53-mutant cancer cells. This not only increases the scope for combining MDM2/MDMX inhibitors in the treatment of p53-mutant cancers but could also be exploited in treatment regimens where p53 is mutated after administration of MDM2 antagonists.

\subsection{Scope of thesis}

This project attempts to enumerate three ways by which targeting MDM2 in the clinics can be improved (Figure 2 10).

1) Adapting the purpose of the drug. MDM2 inhibitors have been exploited for targeting tumor cells that retain wild-type p53. In this study, we used them to protect normal cells against the side effects of drugs causing replicative stress such as WEE1 inhibition and gemcitabine [176].

2) Combination with other drugs. In this case, we combined inhibitors of MDM2 with inhibitors of another negative regulator of p53, namely WIP1, and studied the impact of p53 activation in tumor cells. Indeed, upon combination, we observed that p53 was activated to a greater extent and induced a senescent phenotype [177].

3) Targeting the correct population of tumors. MDM2 has been an attractive target for liposarcomas due to amplification of the gene. However, as mentioned, there has been no

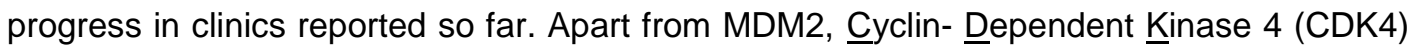
is frequently co-amplified in these cancers ( 90\%). CDK4 is a Ser/Thr kinase that phosphorylates its main substrate $\mathrm{Rb}$ [178]. This prevents the interaction of Rb with E2F1; thereby allowing cells to progress from the $G 1$ to S-phase of the cell-cycle [179, 180]. Targeting MDM2 and CDK4 in combination seemed to be a plausible strategy to eliminate these cancers. Our studies indicated that this was untrue. We observed drug antagonism with respect to cell viability and p53 activity upon combination and discovered a novel interaction partner of p53, namely the CDK4-cyclin D1 complex. 


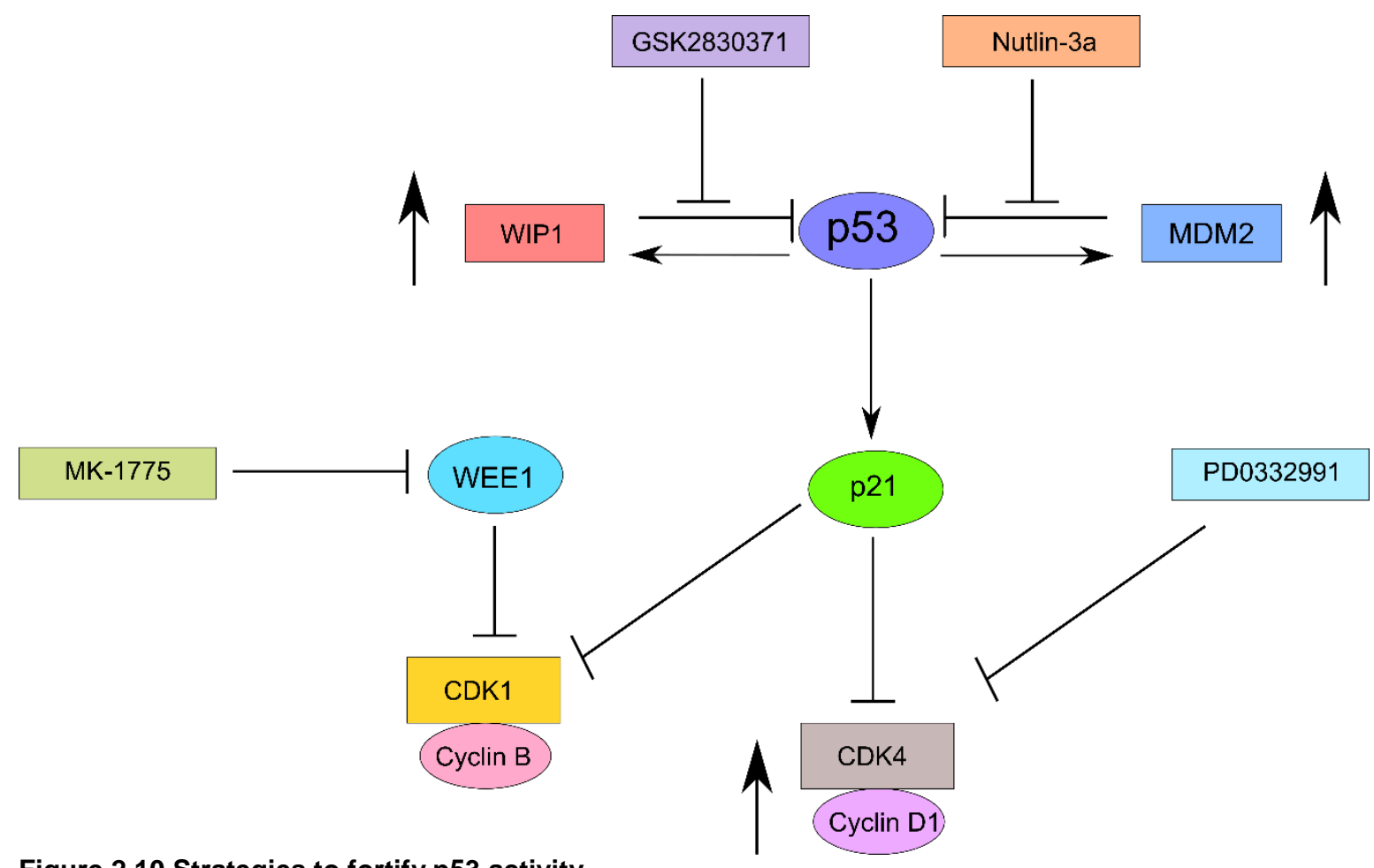

Figure 210 Strategies to fortify p53 activity.

In this thesis, we used three different strategies to target p53. We used inhibitors to MDM2-p53, WIP1-p53, and CDK4/6-cyclin D; thereby enabling us to understand the interplay of these combinations and the resulting outcome of the cells. 


\section{RESULTS}

\subsection{Publication I}

\section{MDM2 inhibition confers protection of p53-proficient cells from the cytotoxic effects of WEE1 inhibitors}

\section{Citation}

Yizhu Li*, Priyanka Saini", Anusha Sriraman*, and Matthias Dobbelstein"

* equal first authors

MDM2 inhibition confers protection of p53-proficient cells from the cytotoxic effects of WEE1 inhibitors

Oncotarget. 2015 Oct 20;6(32):32339-52. doi: 10.18632/oncotarget.5891.

http://www.oncotarget.com/index.php?journal=oncotarget\&page=article\&op=view\&path[]=5891\&pubmedlinkout $=1$

\section{Own contribution:}

Conducted experiments and data analyses for Figures 1A (ii-iv), 2A (ii), 2D, 3B, 4C and Supplemental figure 1. Involved in figure arrangement and contributions in writing and revising the manuscript. 


\title{
Mdm2 inhibition confers protection of p53-proficient cells from the cytotoxic effects of Wee 1 inhibitors
}

\author{
Yizhu Li, ${ }^{1,}$, Priyanka Saini, ${ }^{1,}$, Anusha Sriraman ${ }^{1, *}$ and Matthias Dobbelstein ${ }^{1}$ \\ ${ }^{1}$ Institute of Molecular Oncology, Göttingen Centre of Molecular Biosciences (GZMB), Faculty of Medicine, University of \\ Göttingen, Göttingen, Germany \\ * These authors are equal first authors
}

Correspondence to: Matthias Dobbelstein, email: mdobbel@uni-goettingen.de

Keywords: Wee1, Mdm2, p53, gemcitabine, premature mitosis

Received: June 04, $2015 \quad$ Accepted: September 20, $2015 \quad$ Published: September 29, 2015

This is an open-access article distributed under the terms of the Creative Commons Attribution License, which permits unrestricted use, distribution, and reproduction in any medium, provided the original author and source are credited.

\section{ABSTRACT}

Pharmacological inhibition of the cell cycle regulatory kinase Wee1 represents a promising strategy to eliminate cancer cells. Wee1 inhibitors cooperate with chemotherapeutics, e. g. nucleoside analogues, pushing malignant cells from $\mathbf{S}$ phase towards premature mitosis and death. However, considerable toxicities are observed in preclinical and clinical trials. A high proportion of tumor cells can be distinguished from all other cells of a patient's body by inactivating mutations in the tumor suppressor p53. Here we set out to develop an approach for the selective protection of p53-proficient cells against the cytotoxic effects of Wee1 inhibitors. We pretreated such cells with Nutlin-3a, a prototype inhibitor of the p53-antagonist Mdm2. The resulting transient cell cycle arrest effectively increased the survival of cells that were subsequently treated with combinations of the Wee1 inhibitor MK1775 and/or the nucleoside analogue gemcitabine. In this constellation, Nutlin-3a reduced caspase activation and diminished the phosphorylation of Histone $2 A X$, an indicator of the DNA damage response. Both effects were strictly dependent on the presence of p53. Moreover, Nutlin pre-treatment reduced the fraction of cells that were undergoing premature mitosis in response to Wee1 inhibition. We conclude that the pre-activation of p53 through Mdm2 antagonists serves as a viable option to selectively protect p53-proficient cells against the cytotoxic effects of Wee1 inhibitors, especially when combined with a nucleoside analogue. Thus, Mdm2 antagonists might prove useful to avoid unwanted side effects of Wee1 inhibitors. On the other hand, when a tumor contains wild type p53, care should be taken not to induce its activity before applying Wee1 inhibitors.

\section{INTRODUCTION}

Inhibitors of the kinase Wee1 are capable of inducing cancer cell death with high efficiency, in particular when combined with chemotherapeutics such as nucleoside analogues [1] or platinum compounds [2]. In particular, the Wee1 inhibitor MK-1775 has been found efficient to eliminate a number of cancer cell species [3, 4], and it is currently evaluated in numerous clinical trials ( [5] and 21 entries to clinicaltrial.gov).

Wee1 is a cell cycle regulatory kinase. It phosphorylates and thereby inactivates the downstream cyclin dependent kinases (CDKs) 1 and $2[6,7]$ while the cell replicates its DNA (i. e. in S phase) [8]. This suppression of CDKs ensures that the cell will first complete the replication of the entire genome before moving on to mitosis. Removing Weel by siRNA, or inhibiting Wee1 by small compounds, results in the premature onset of mitosis, thereby increasing cell death $[3,4]$.

Wee1 inhibition can be regarded as a way to exploit replicative stress for cancer treatment, as we have reviewed recently [9]. Tumor cells often display impaired abilities to ensure a smooth and uninterrupted replication of their DNA. Further increasing this stress 
situation represents a viable strategy of cancer therapy. This can be achieved by classical chemotherapeutics, e. g. nucleoside analogues. Representatives of this class include gemcitabine (2', 2'-difluorodeoxycytidine, $\mathrm{dFdC}$ ), an analogue of deoxycytidine. Gemcitabine inhibits ribonucleotide reductase, thus leading to a shortage and imbalance of available deoxyribonucleotide triphosphates. Moreover, it is incorporated into newly synthesized DNA, leading to torsional stress and replication fork stalling [10]. Interfering with the replication machinery is one example of targeting tumor-supportive cellular machineries for cancer treatment, as reviewed recently [11].

Weel inhibition and the consecutive activation of CDK1 can exacerbate replicative stress by at least three mechanisms. Firstly, we have recently identified a mechanism that leads from Weel inhibition to the inactivation of Chk1, a key enzyme required to re-enable DNA replication in the context of replicative stress [12]. Moreover, Wee1 inhibition increases nucleotide consumption and thereby increases replicative stress [13]. On top of this, however, Weel inhibition, by enabling premature CDK activity during $\mathrm{S}$ phase, promotes mitosis despite the fact that their DNA is incompletely replicated [1]. This will either disable the completion of mitosis, resulting in catastrophic death, or otherwise lead to the formation of two daughter cells with gross genetic deletions, again precluding survival.

Despite the encouraging preclinical and clinical findings, Wee1 inhibitors have not achieved clinical approval yet. One of the problems faced when evaluating these drug candidates consisted in the toxicity that limited the amount of inhibitors that can be safely administered. Such dose limiting toxicities include myelosuppression and tachyarrhythmia [5]. In other words, a better distinction between normal cells and the tumor cells in a patient's body is required, and the cytotoxic effects should be limited to the tumor cells as much as possible.

The most frequent genetic difference between tumor cells and normal cells consists in mutations within the gene TP53, encoding the tumor suppressor and transcription factor p53 [14]. When activated, e. g. by phosphorylation through DNA damage-induced kinases, p53 induces the expression of genes that induce cell cycle arrest in G1 or G2. Strong p53 activation, e. g. by excessive DNA damage, can also induce cell death, most notably by apoptosis [15]. More than 50\% of all tumors, however, carry an inactivating mutation in TP53. This typically disables the encoded p53 protein from binding to its cognate promoter sequences, precluding transactivation. In these cases, pharmacological activation of p53 will only pertain to normal cells but not to tumor cells.

p53 activity is kept under tight control by its antagonist Mdm2. Mdm2 binds and inactivates p53, and on top of this, it acts as an E3 ubiquitin ligase to target p53 for proteasomal degradation. The synthesis of Mdm2 is induced by $\mathrm{p} 53$, leading to a negative regulatory feedback loop. Small molecule inhibitors have been developed to bind Mdm2, precluding p53 from binding to the same site. As a result, these drugs can be used to augment the levels of active p53, even in the absence of DNA damage [16]. The prototype compound of this kind has been termed Nutlin-3a [17], but several similar drug candidates have been developed since and are currently under evaluation in clinical studies [18].

While mostly regarded as an inhibitor of cell survival, p53 can also be employed to protect cells. To this end, Mdm2 inhibitors can be employed to activate p53. We have first described the protective effect of Mdm2 inhibition in the case of nucleoside analogues, e. g. gemcitabine [19]. Since p53 arrests cells in G1 or G2, few cells replicate their DNA upon p53 activation by Mdm2 inhibitors, and nucleoside analogues can no longer be incorporated into nascent DNA strands. As a result, the cells become resistant to treatment with nucleoside analogues. When both drugs are washed off, the cells can resume proliferation with only short delays. An analogous approach was used to achieve protection against taxanes, i. e. drugs that target the mitotic spindle. Pre-treatment with Nutlin-3a precludes cells from entering mitosis, the most vulnerable phase of cells in the face of taxanes, and it thus ensures cell survival [20]. The protective effect of Mdm2 against mitotic inhibitors is active for several days and can be further enhanced by rapamycin [21]. Thus, p53 activation can provide protection of p53-proficient cells against specific classes of drugs. This strategy dates back to the beginning of the millennium, when low-dose DNAdamaging agents provided protection against microtubuleactive drugs through p53 [22, 23], a principle termed cyclotherapy [24].

Here we show that Mdm2 inactivation successfully protects p53-proficient cells against the cytotoxic effects of Wee1 inhibition. When p53 is pre-activated, Wee1 inhibitors alone or in combination with gemcitabine no longer prevent long term proliferation and survival. Mechanistically, p53-activation keeps cells from the lethal premature mitosis that is otherwise induced by Wee1 inhibition.

\section{RESULTS}

\section{Mdm2 inhibition allows cells to survive the treatment with Wee1 inhibitor and/or gemcitabine}

To assess whether pre-treatment with an Mdm2 inhibitor affects the survival of p53-proficient cells, we first treated U2OS cells (human osteosarcoma, p53 wild type) with Nutlin-3a, the prototype pharmacological antagonist that binds to $\mathrm{Mdm} 2$ and precludes its interaction with p53 [17]. After a 24 hrs incubation time, the cells were treated with gemcitabine and/or the Weel inhibitor 
MK-1775 (termed Wee1i from here on) for another 24 hrs, while maintaining the concentration of Nutlin-3a (simply termed Nutlin from here on) as before. For each drug, control experiments using the DMSO solvent were performed in parallel. Subsequently, all drugs were washed off, followed by further incubation in regular cell culture media. For twelve days, the cell density was monitored by transmission light microscopy and automated image analysis (Fig. 1A). Gemcitabine alone did not lead to a strong impairment of cell proliferation, and also Weeli
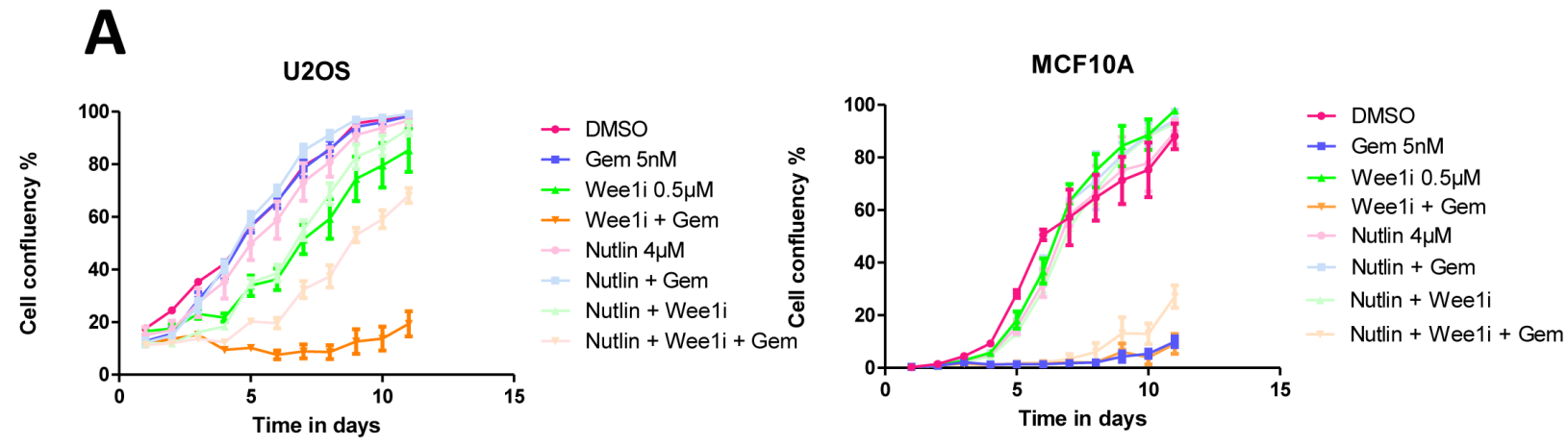

HCT116 p53+l+
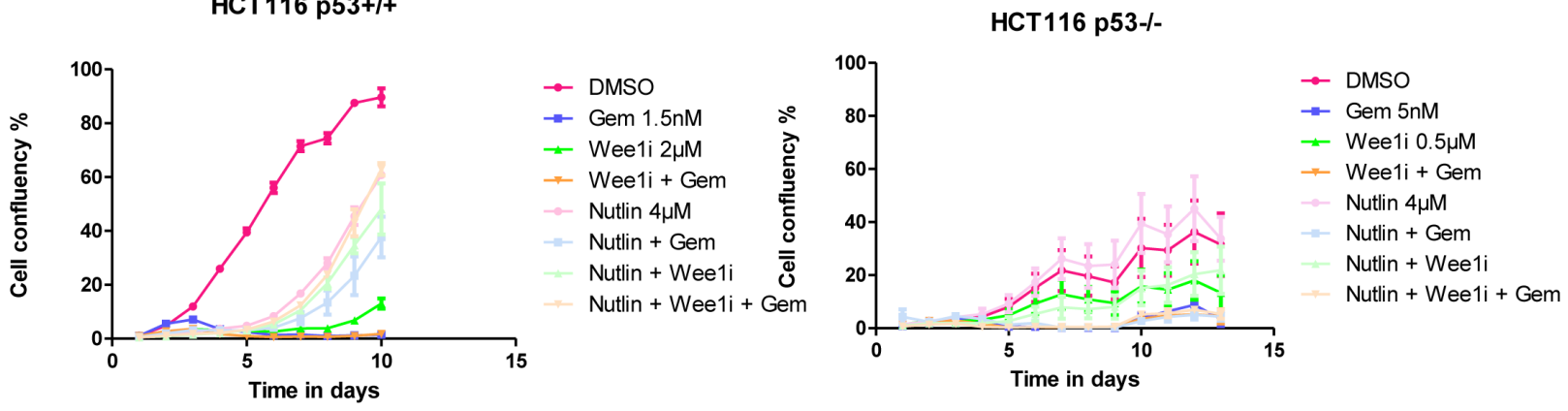

B

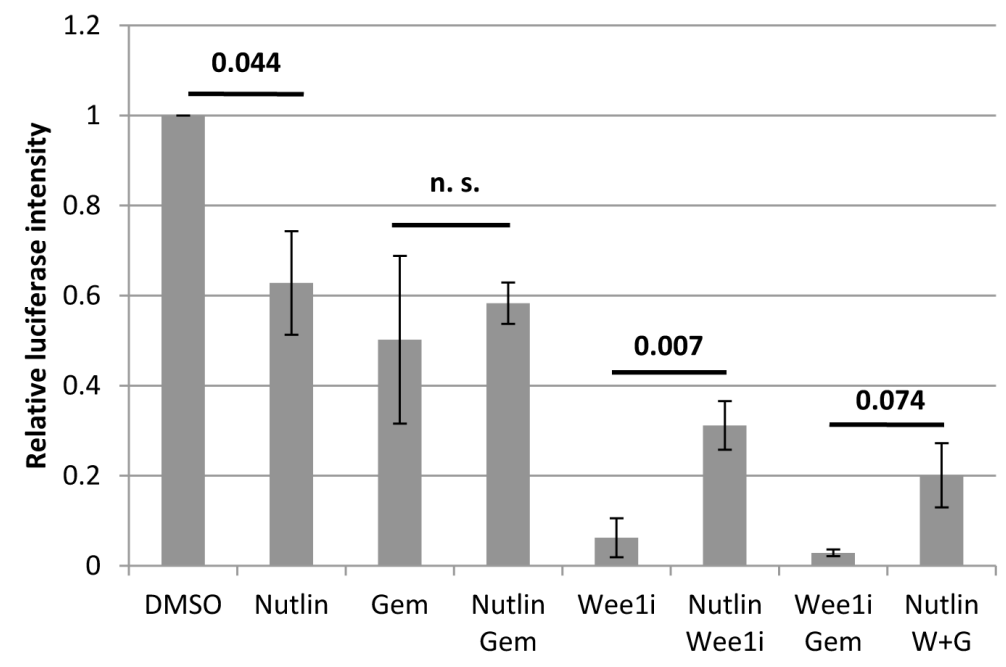

Figure 1: Nutlin protects cells against Wee1 inhibition and/or gemcitabine. A. U2OS, MCF10A, HCT116 p53+/+, and HCT116 p53-/- cells were treated with Nutlin-3a for 24 hrs, followed by treatment with MK-1775 (Wee1i), gemcitabine and continuous incubation with Nutlin, at the indicated drug concentrations. After another $24 \mathrm{hrs}$, all drugs were removed and fresh medium was added. Cells were incubated for 8-13 days and confluency was measured each day using brightfield microscopy (Celigo cell cytometer). Error bars represent the SD, $\mathrm{n}=3$ (triplicate experiments). B. U2OS cells were treated with $8 \mu \mathrm{M}$ Nutlin for $24 \mathrm{hrs}$, followed by treatment with $1 \mu \mathrm{M}$ Weeli and $300 \mathrm{nM}$ gemcitabine, along with continuous treatment with $8 \mu \mathrm{M}$ Nutlin. At $72 \mathrm{hrs}$, the cells were lysed using the CellTiter-GloßReagent, and cell viability was measured via an ATP-dependent luciferase signal. Student's T-test p-values are stated above the horizontal bars. Error bars represent the $\mathrm{SE}, \mathrm{n}=3$. 
alone only moderately prevented cell growth. When applied together at the same concentrations, however, the two drugs strongly reduced the appearance of proliferating cells, essentially preventing the formation of a confluent layer, confirming the synergy that was described before [12, 25-28]. Strikingly, the pre-treatment with Nutlin rescued the proliferation of cells that were treated with Wee1i alone, and even more strongly reversed the effect of Weeli and gemcitabine in combination. Parallel experiments were performed with the non-transformed cell line MCF10A. Interestingly this cell line was largely resistant to Wee1 inhibition. However, the cells responded to Gemcitabine or the combination of Gemcitabine with Weeli, and in both cases, this effect was alleviated by Nutlin. To define the role of p53 in the protection by Nutlin, we employed HCT116 cells, a colon cancerderived cell line that had been engineered to either contain or lack wild type p53 [36]. In the case of HCT116 p53+/+ cells, we observed that cell proliferation on treatment with gemcitabine or Weeli, and also upon co-treatment with Wee1i, was strongly reduced. However, in combination with Nutlin, we observed a rescue in cellular proliferation. In HCT116 p53-/- cells, however, no such rescue by Nutlin was observed. Thus, the protective effect of Nutlin is $\mathrm{p} 53$-dependent. We conclude that pre-treatment with Nutlin has an intense protective effect and allows cells to survive the treatment with Wee1i, alone or in combination with gemcitabine.

Next, we investigated whether Nutlin pre-treatment also affects immediate cell viability when cells are exposed to gemcitabine and/or Wee1i. To test this, we treated $\mathrm{U} 2 \mathrm{OS}$ cells as above, followed by a $72 \mathrm{hrs}$ incubation and a viability assay based on the determination of cellular ATP levels by luciferase (Fig. 1B). All three drugs gemcitabine, Nutlin, and Weeli - led to a reduction in viability, presumably through a combination of cell death and arrested proliferation. Weeli, alone or in combination with gemcitabine, reduced viability most strongly. Importantly, however, Nutlin rescued the viability of Weeli-treated cells, with or without gemcitabine. Thus, Nutlin pre-treatment strongly protects cells from the induction of death by Weeli.

\section{Mdm2 inhibition attenuates caspase activity and the phosphorylation of Histone2 $\mathrm{AX}$ in response to Wee1 inhibition}

Weeli exerts its toxic effects, at least in part, by inducing a DNA damage response $[8,13]$ and apoptosis [29]. We therefore tested whether Nutlin pre-treatment reduces any or both of these responses. U2OS cells were pre-treated with Nutlin or the DMSO solvent, followed by gemcitabine and/or Wee1i. Subsequently, the cleavage of poly ADP-ribose polymerase (PARP), a bona fide caspase substrate [30], was monitored by immunoblot analysis; we also probed the phosphorylation of Histone2AX $(\gamma \mathrm{H} 2 \mathrm{AX})$, a hallmark of the DNA damage response [31] (Fig. 2A). Weeli induced PARP cleavage as well as a strong accumulation of $\gamma \mathrm{H} 2 \mathrm{AX}$ in the presence or absence of gemcitabine, as reported previously [12]. Notably, however, both responses were clearly reduced when the cells had been pre-treated with Nutlin. Similar results were obtained when blocking caspase activities by the cell-permeant pan caspase inhibitor Z-VAD-FMK, suggesting that $\gamma \mathrm{H} 2 \mathrm{AX}$ levels represent the direct result of a DNA damage response, not an indirect consequence of caspase activation. To confirm the reduction in $\gamma \mathrm{H} 2 \mathrm{AX}$ independently, we assessed its accumulation by immunofluorescence and subsequent digital image analysis (Fig. 2B and 2C), as described [32]. We observed the accumulation of $\gamma \mathrm{H} 2 \mathrm{AX}$ upon treatment with gemcitabine and Wee1i, alone or in combination. In each case, however, Nutlin pre-treatment led to a highly significant reduction in the accumulation of $\gamma \mathrm{H} 2 \mathrm{AX}$. Finally, we assessed the activity of caspases in cell lysates obtained from U2OS cells after drug treatment. We observed increased activities in samples treated with Wee1i, alone or and in combination with Gemcitabine; again, however, this was rescued upon pre-treatment with Nutlin (Fig. 2D; Suppl. Fig. 1). In control samples treated with Z-VAD-FMK, no caspase activity was observed, validating the assay. Taken together, Mdm2 inhibition attenuates both the activation of caspases as well as DNA response signaling upon inhibition of Wee1.

\section{The presence of p53 is required for the protective effect of Nutlin-3a against Wee1 inhibition}

Mdm2 is mostly known for its impact on p53, but additional activities of Mdm2 have been reported [33], and some of them may be affected by Mdm2 antagonists as well. To assess whether Nutlin antagonizes Weeli through p53, we first assessed whether it increases the levels of p53 and the product of a target gene, CDKN1A/ p21 [34], in U2OS cells (Fig. 3A). As expected, Nutlin led to the accumulation of p53 as well as p21. Importantly, neither the subsequent treatment with gemcitabine nor the exposure to Weeli led to any gross changes in the levels of p53 or p 21 when cells had been pre-treated with Nutlin. We did, however, observe the accumulation of p53 but not $\mathrm{p} 21$ when the cells were treated with gemcitabine and/or Wee1i alone. This is in agreement with previous analyses indicating that DNA damage (as observed by $\gamma \mathrm{H} 2 \mathrm{AX}$ accumulation) during $\mathrm{S}$ phase stabilizes $\mathrm{p} 53$ but nonetheless attenuates the induction of p21 [35]. In any case, the effects of Nutlin on p53 levels and activity were not compromised by gemcitabine and/or Weeli.

To define the role of p53 in the negative impact of Nutlin on $\gamma \mathrm{H} 2 \mathrm{AX}$ accumulation, we transfected U2OS cells with siRNA to p53. This knockdown abolished the 

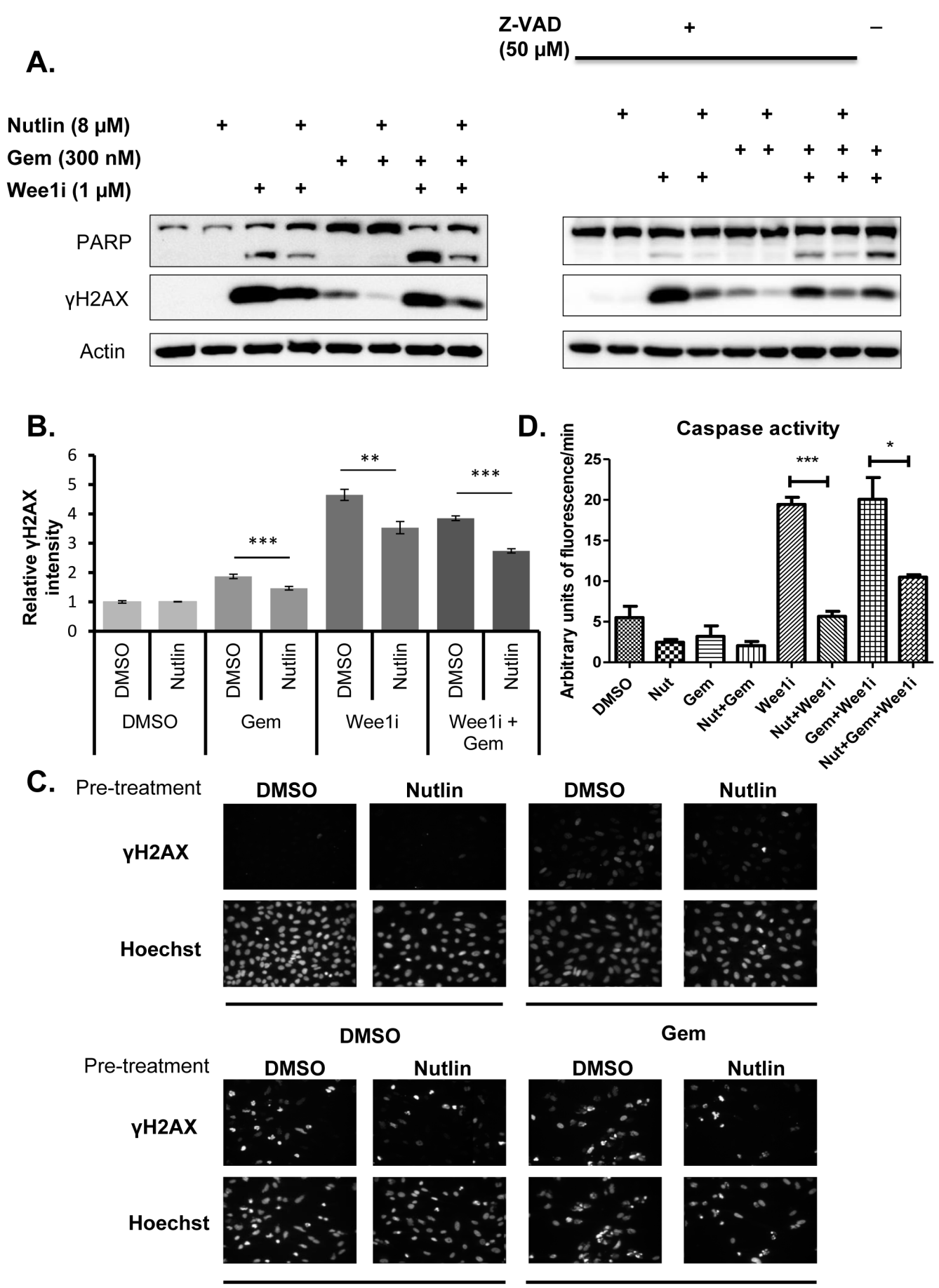

Wee1i
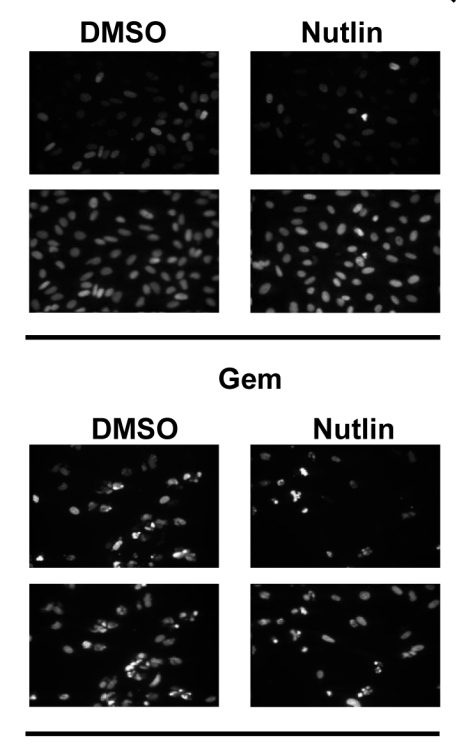

Wee1i + Gem

Figure 2: Nutlin prevents caspase activation and $\gamma \mathrm{H} 2 \mathrm{AX}$ accumulation in response to Wee1 inhibitor and/or gemcitabine. A. U2OS cells were treated with $8 \mu \mathrm{M}$ Nutlin for $24 \mathrm{hrs}$, followed by treatment with $1 \mu \mathrm{M}$ Weel inhibitor, $300 \mathrm{nM}$ gemcitabine, and/or $8 \mu \mathrm{M}$ Nutlin in the absence and presence of 50 $\mu \mathrm{M}$ ZVAD-FMK for another $24 \mathrm{hrs}$. Cells were harvested and immunoblot analysis was performed to detect poly-ADP ribose polymerase (PARP) and $\gamma$ H2AX. B., C. U2OS cells were treated as in (A). The cells were then fixed and stained for $\gamma \mathrm{H} 2 \mathrm{AX}$ by immunofluorescence. Detection and analysis was performed using automated immunofluorescence microscopy (BD Pathway). Figure panel (B) shows images of $\gamma \mathrm{H} 2 \mathrm{AX}$ staining for each treatment condition. Quantitation of $\gamma \mathrm{H} 2 \mathrm{AX}$ intensities was done using the $\mathrm{BD}$ pathway analysis tool and depicted in figure panel (C). Error bars represent the SD, $\mathrm{n}=3$. D. U2OS cells were treated with $8 \mu \mathrm{M}$ Nutlin for $24 \mathrm{hrs}$, followed by treatment with $1 \mu \mathrm{M}$ Wee1 inhibitor, $300 \mathrm{nM}$ gemcitabine, $8 \mu \mathrm{M}$ Nutlin in the absence and presence (Supplementary

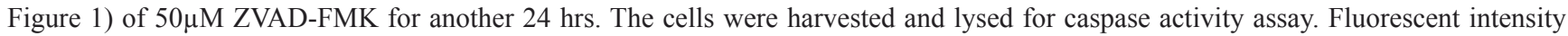
measurements were obtained for each treatment. The activity (arbitrary units of fluorescence/min) was calculated for each treatment at the linear part of the curve (cf. Supplementary Figure 1). Error bars represent the S.D, n=3. 
influence of Nutlin on $\gamma \mathrm{H} 2 \mathrm{AX}$ (Fig. 3B), indicating that the protective effective of Nutlin against Weeli depends on $\mathrm{p} 53$.

Next, we assessed the protective effect of Nutlin in a system of isogenic cells that only differ in their p53 status. HCT 116 cells that either contained or lacked wild type p53 [36] were employed for this purpose. Again, these cells were pre-treated with Nutlin, followed by gemcitabine and/or Weeli, and the accumulation of cleaved PARP as well as $\gamma \mathrm{H} 2 \mathrm{AX}$ was assessed by immunoblot analysis (Fig. 3C). In the case of cells containing wild type p53, Nutlin prevented both caspase activity and the DNA damage response, similar to U2OS cells. When TP53 had been deleted, however, Nutlin did not influence any of these responses. In conclusion, p53 is strictly required for the protective effects of Nutlin against Wee1i. Thus, p53 activity is the principal mediator of this protection.

A.

U2OS

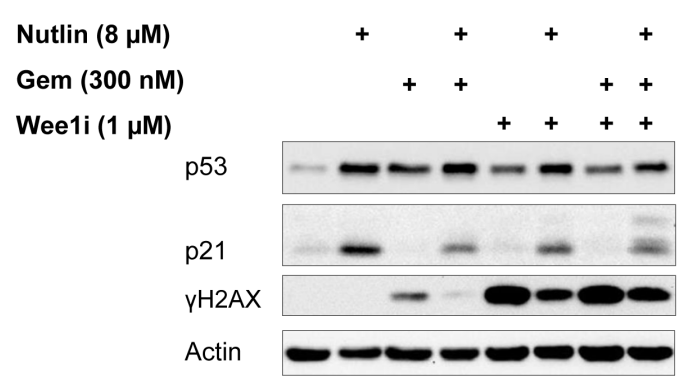

B.

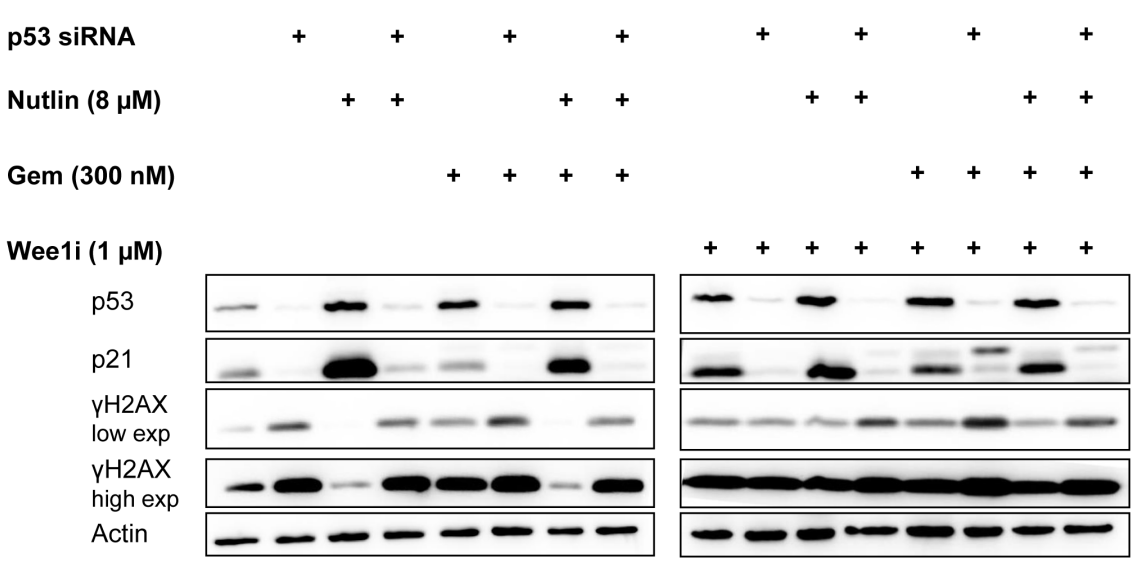

C.

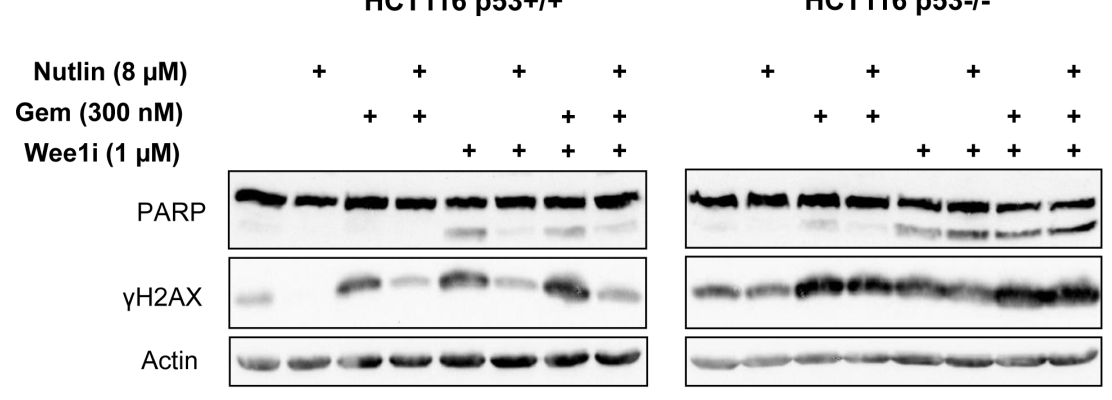

Figure 3: p53 is required for the protective effect of Nutlin. A. U2OS cells were treated with $8 \mu \mathrm{M}$ Nutlin for 24 hrs, followed by treatment with $1 \mu \mathrm{M}$ Wee1 inhibitor, $300 \mathrm{nM}$ gemcitabine and $8 \mu \mathrm{M}$ Nutlin for another $24 \mathrm{hrs}$ as indicated. Cells were harvested and immunoblot analysis was performed to detect p53 and its target gene product p21. B. U2OS cells transfected with siRNA were treated with $8 \mu \mathrm{M}$ Nutlin at $24 \mathrm{hrs}$ post-transfection, then incubated for additional $24 \mathrm{hrs}$, followed by treatment with $1 \mu \mathrm{M}$ Wee1 inhibitor, 300nM gemcitabine and $8 \mu \mathrm{M}$ Nutlin for another $24 \mathrm{hrs}$ as indicated. Immunoblot analysis was performed to detect p53 and its target gene product p21, as well as $\gamma \mathrm{H} 2 \mathrm{AX}$. C. An isogenic pair of HCT116 cells with or without a targeted deletion of TP53 was pre-treated with $8 \mu \mathrm{M}$ Nutlin for $24 \mathrm{hrs}$, followed by treatment with Wee1 inhibitor, gemcitabine and Nutlin for another $24 \mathrm{hrs}$. Cells were harvested and subjected to immunoblot analysis to detect PARP and $\gamma \mathrm{H} 2 \mathrm{AX}$. 


\section{Nutlin-3a prevents the accumulation of cells in premature mitosis when exposed to Wee1 inhibitor}

Wee1 acts to prevent the premature onset of mitosis, and its inhibition is known to trigger chromosome condensation and cell division, even before the replication of cellular DNA is complete. This condition - often referred to as premature mitosis - leads to a catastrophic situation and cell death [1]. Premature mitosis is even further enhanced when Wee1 inhibitors are combined with DNA-damaging agents, such as nucleoside analogues or platinum compounds [12, 25, 26, 28]. On the other hand, p53 often prevents even the entry of cells into S phase, or otherwise acts to block the transition into mitosis [37]. We therefore tested whether Mdm2 inhibition and p53 activation might prevent premature mitosis when cells are exposed to Wee1i. Firstly, we determined the amount of U2OS cells actively synthesizing DNA upon pre-treatment with Nutlin and/or subsequent treatment with Weeli (Fig. 4A). Nutlin strongly reduced the number of cells in $\mathrm{S}$ phase, as determined by the incorporation of the labeling nucleoside analogue 5-ethynyl-2'-deoxyuridine (EdU) andreported previously [19]. Notably, the treatment with Weeli also reduced the amount of EdU-incorporating cells, presumably due to interruptions in $\mathrm{S}$ phase. However, even in this situation, Nutlin further reduced the percentage of DNA-synthesizing cells, arguing that Nutlin keeps cells out of S phase regardless of subsequent Wee1i treatment. And indeed, propidium iodide staining of the cells revealed that Nutlinpretreated cells were largely accumulating with a DNA content corresponding to G1 or G2/M, regardless of their subsequent treatment (Suppl. Fig. 2).

Next, we compared the extent of entry into mitosis when U2OS cells were treated with Wee1i and/ or gemcitabine, in the presence or absence of Nutlin pre-treatment. Weeli, alone or in combination with gemcitabine, strongly augmented the accumulation of Histone3 (H3) that was phosphorylated at Serine 10, a marker of cells in mitosis [38] (Fig. 4B). Of note, however, Nutlin pre-treatment reduced the phospho-H3 signal in all combinations of Weeli and gemcitabine. Thus, Nutlinpretreatment reduces the accumulation of mitotic cells upon exposure to Weeli.

Similar investigations were carried out in isogenic HCT116 cells with or without p53. Again, these cells were pre-treated with Nutlin, followed by gemcitabine and/or Wee1i, and the accumulation of Histone 3 (H3) that was phosphorylated at Serine 10 was assessed by immunoblot analysis (Fig. 4C). As expected, Weeli increased the levels of phospho-H3, whereas Nutlin prevented this accumulation. Importantly, however, this was only observed in p53-proficient cells. When p53 was absent, Wee1 inhibition still induced phospho-H3 accumulation, but this was not affected by Nutlin.

Immunoblot analysis does not distinguish between regular and premature mitosis. In order to find out how Nutlin affects the accumulation of cells that prematurely enter cell division, we treated U2OS cells with combinations of the three drugs, followed by twodimensional flow cytometry, quantifying both the DNA content and the amount of phosphorylated $\mathrm{H} 3$ in every cell (Fig. 4D and 4E). Cells with a DNA content below $4 \mathrm{~N}$ but a phospho-H3 content above the baseline were considered prematurely mitotic. As expected, Weeli led to the accumulation of cells in premature mitosis, especially when combined with gemcitabine. However, this number was strongly reduced when pre-treating the cells with Nutlin. We conclude that Nutlin prevents premature mitosis in cells that are confronted with Weeli, alone or in combination with gemcitabine. We propose that this mechanism is at least partially responsible for the protection of Wee1i-treated cells against Nutlin.

\section{DISCUSSION}

According to our results, the pharmacological inhibition of Mdm2 prevents the toxicity of a Wee1 inhibitor, in the presence or absence of the nucleoside analogue gemcitabine. In agreement, the Mdm2-inhibitor Nutlin prevents the accumulation of phosphorylated H2AX and the activation of apoptosis in response to Weeli. As expected, this protective effect conferred by Nutlin strictly requires the presence of p53. Mechanistically, p53 diminishes the onset of premature mitosis by Weeli and/or gemcitabine. We propose that Nutlin, by inducing the CDK inhibitor p21, interferes with G1-S transition and thus prevents replicative stress in the first place. In addition, p21 also attenuates CDK1 activity [39] and may thereby diminish premature mitosis even in those cells that nonetheless entered S phase (Fig. 5).

These observations are suggesting two major conclusions. Firstly, the therapeutic effect of Wee1 inhibitors may be reduced or even abolished if wild type p53 is activated prior to Wee1 inhibition. This not only argues against the combination of Mdm2 inhibitors with Wee1 inhibitors to treat p53-wildtype cancers. Rather, p53 is activated by most DNA-damaging therapeutic regimens, including irradiation and chemotherapy, e. g. by platinum compounds, topoisomerase inhibitors, alkylating agents, and many others [40]. When combining any of these chemotherapeutics with Wee1 inhibitors, it appears advisable to administer the Weel inhibitor before or at least simultaneously with chemotherapy, but not shortly after it. Otherwise, it is conceivable that the pre-activated p53 will interfere with cell cycle progression and thus with the efficacy of Wee1 inhibitors. In contrast, the presence of wild type but not pre-activated p53 does not seem to preclude the cytotoxic activity of a Weel inhibitor [41]. Notably, these considerations only applies to tumors that 
A.

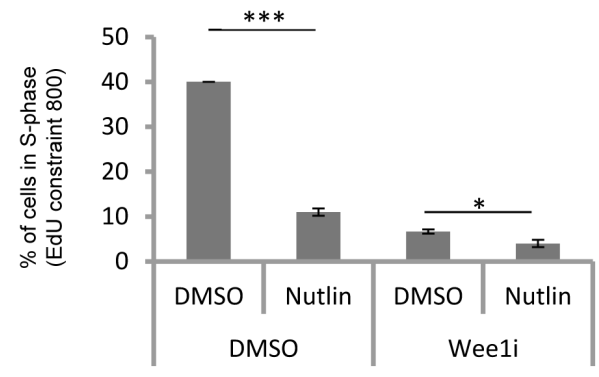

B.

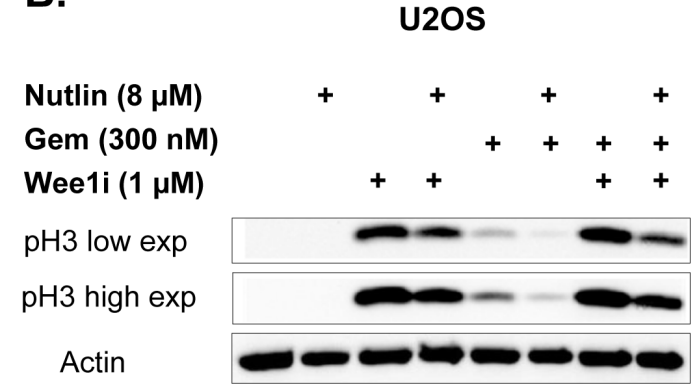

HCT 116 p53-/-
C.

HCT116 p53+/+

HCT 116 p53-

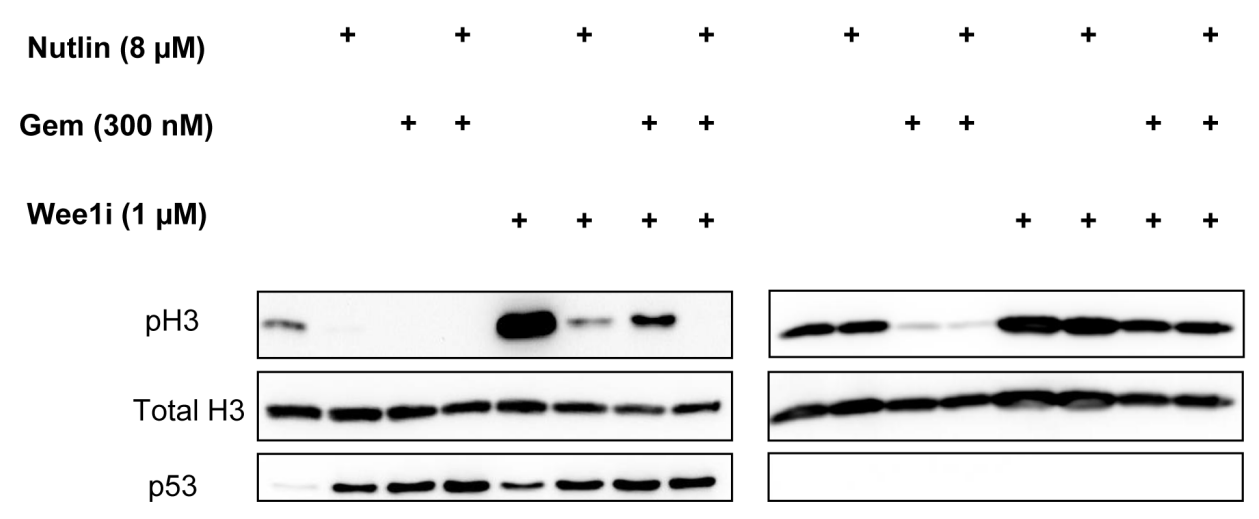

D.

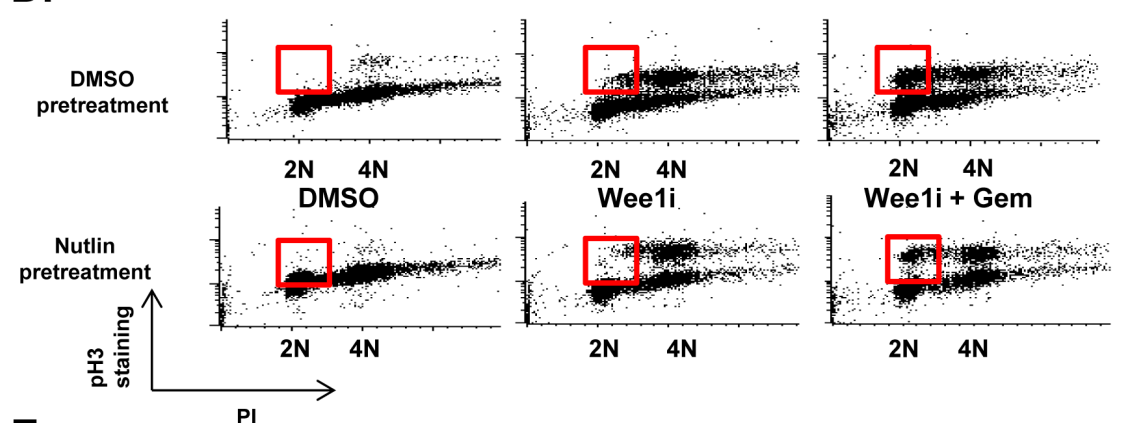

E.
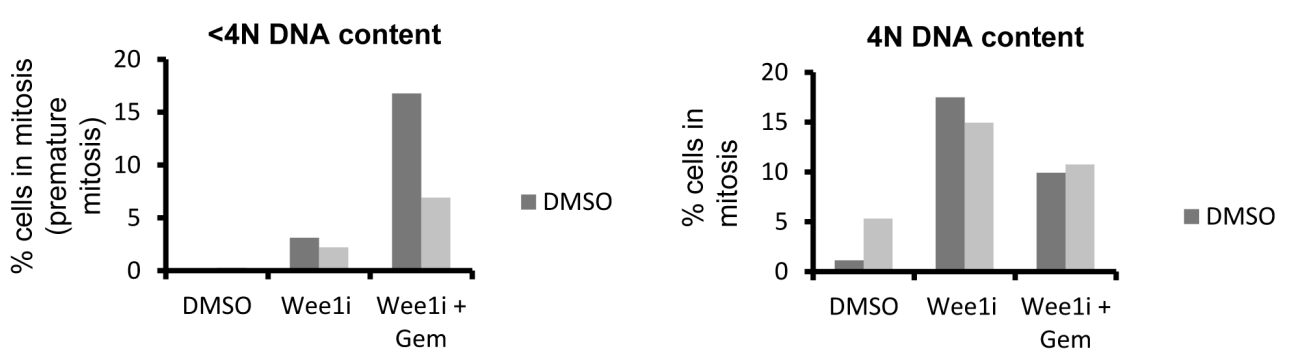

Figure 4: p53 prevents accumulation of cells in premature mitosis. A. U2OS cells were treated with $8 \mu \mathrm{M}$ Nutlin for 24 hrs, followed by treatment with $1 \mu \mathrm{M}$ Wee 1 inhibitor and $8 \mu \mathrm{M}$ Nutlin for another 24 hrs. Two hours before fixation, $5 \mu \mathrm{M}$ of 5-Ethynyl-2'deoxyuridine (EdU) was added. Afterwards, cells were stained for EdU, and the percentage of cells with EdU staining intensities of 800 unitsor more was plotted. Error bars represent the SD, $\mathrm{n}=3$. B. U2OS cells were treated with $8 \mu \mathrm{M}$ Nutlin for $24 \mathrm{hrs}$, followed by treatment with $1 \mu \mathrm{M}$ Wee1 inhibitor, 300nM gemcitabine and $8 \mu \mathrm{M}$ Nutlin for another $24 \mathrm{hrs}$. Immunoblot analysis was performed to detect Histone3 with a phosphorylation at Serine 10, a hallmark of mitosis. C. An isogenic pair of HCT116 cells with or without a targeted deletion of TP53 was pre-treated with $8 \mu \mathrm{M}$ Nutlin-3 for $24 \mathrm{hrs}$, followed by treatment with Weel inhibitor, gemcitabine and Nutlin for another $24 \mathrm{hrs}$. Cells were harvested and subjected to immunoblot analysis to detect Histone $\mathrm{H} 3$ with a phosphorylation at Serine 10, p53, and total histone $\mathrm{H} 3$. D., E. U2OS cells were pre-treated with nutlin-3 as in Fig. 2B and 2C, followed by treatment with $1 \mu \mathrm{M}$ Wee 1 inhibitor, $300 \mathrm{nM}$ gemcitabine and $8 \mu \mathrm{M}$ Nutlin for $8 \mathrm{hrs}$. The cells were fixed, stained for phospho-H3 along with propidiumiodide (PI) labelling, and analyzed by flow cytometry. The red boxes demarcate cells in premature mitosis. Figure panel (D) represents the percentage of cells stained positive for phospho-H3. 
retain wild type p53, thus in about $50 \%$ of all human malignancies.

Secondly, our results suggest a strategy that may ultimately help to prevent unwanted toxicities of Wee1 inhibitors. Such toxicities, e. g. myelosuppression and tachyarrhythmia, have been reported [5] and may currently limit the usefulness of this class of drugs, especially when combining them with conventional chemotherapy, and despite their highly promising anti-cancer activity in preclinical models [2, 25, 26, 28, 42-47]. In those cases where p53 is absent or mutant and thus unable to activate its target genes, the administration of Nutlin or similar Mdm2 inhibitors will not interfere with the efficacy of Wee1 inhibitors against tumor cells, as exemplified by p53-/- HCT116 cells in this study (Fig. 3B). However, normal cells from such patients still contain wild type p53. The reversible activation of p53 by an Mdm2 antagonist can thus be expected to attenuate the toxic effects imposed by Wee1 inhibitors on non-cancerous cells. Thus, besides their use to eliminate cancer cells that contain wild type p53 but hyperactive Mdm2, Mdm2-inhibitors may prove useful as a means to prevent unwanted side effects of Wee1 inhibitors.

Such a use of Mdm2 inhibitors for avoiding the toxicities of cancer treatment is not limited to Wee1inhibitors. Rather, Nutlin and related compounds were suggested to prevent the side effects of other anti-cancer compounds. We have previously found that Nutlin also acts to reduce the toxicities of nucleoside analogues in p53-proficient cells [19], and similar protective effects have been reported for taxanes[20, 48], HDAC inhibitors [49], resveratrol, [50], and other chemotherapeutics [51-56]. Furthermore, the protective effect of Mdm2inhibitors is to be expected for any compound that affects cell survival predominantly in $\mathrm{S}$ or $\mathrm{M}$ phase. This includes inhibitors of Chk1 [57] and ATR [58], which increase replicative stress and promote premature mitosis; when combined with platinum compounds and

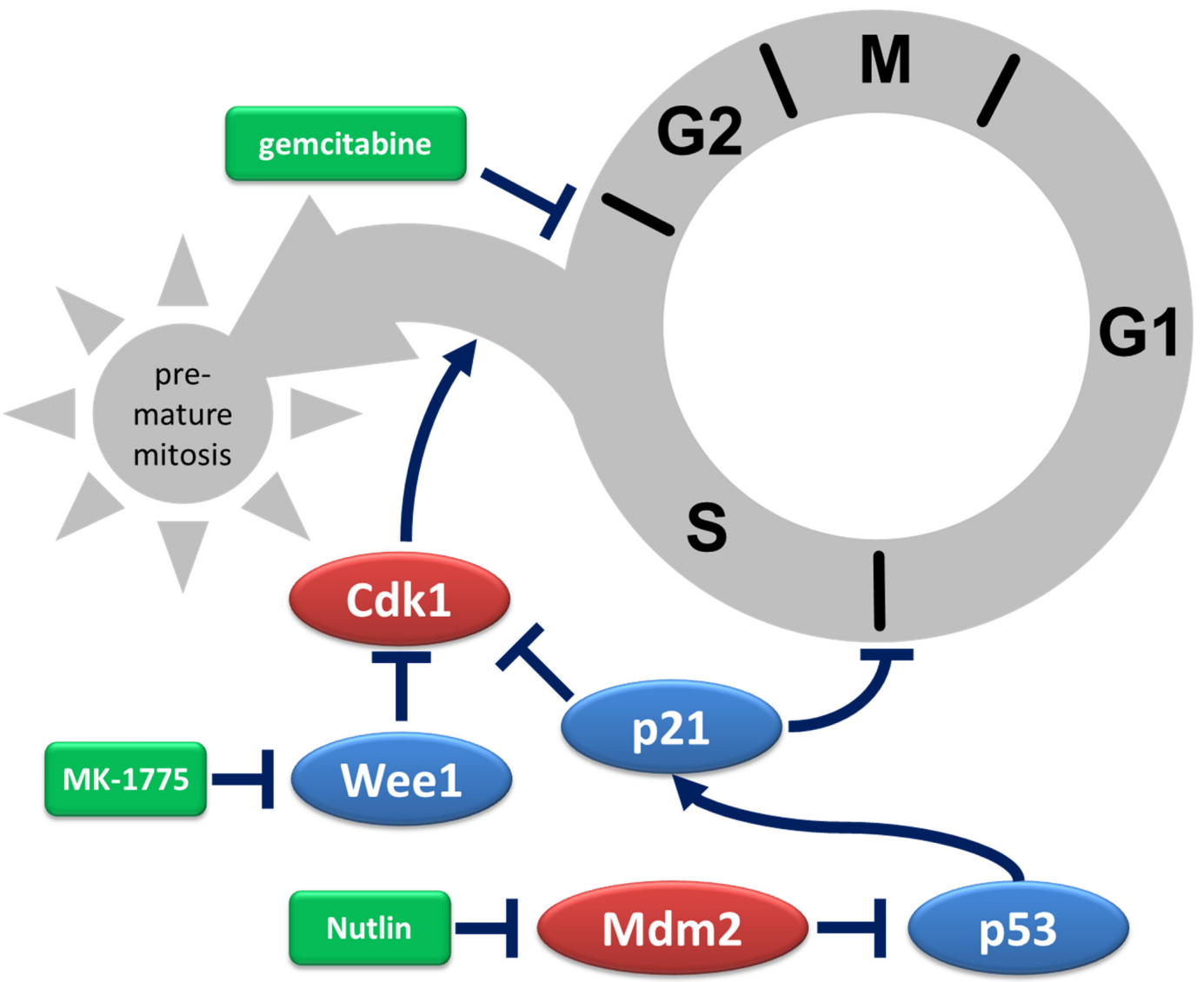

Figure 5: Depiction of protective mechanisms triggered by Mdm2 inhibition. Gemcitabine halts progression through $\mathrm{S}$ phase by interfering with DNA replication. In the presence of Wee1 inhibitors, hyperactive CDK1 triggers premature mitosis despite incomplete DNA replication, usually resulting in cell death. When Mdm2 inhibitors activate p53 and thereby increase the levels of the CDK inhibitor $\mathrm{p} 21$, the transition from G1 to S phase is inhibited. Moreover, CDK1 inhibition by $\mathrm{p} 21$ reduces premature entry in mitosis. Taken together, pre-treatment of $\mathrm{p} 53$-proficient cells with an inhibitor of $\mathrm{Mdm} 2$ attenuates the cytotoxic effects of Weel inhibition. In the scheme, activators of cell cycle progression are depicted in red, inhibitors of cell cycle progression in blue, and drugs in green. Arrows indicate activation, lines that end with a bar indicate inhibition. 
anthracyclines, the same was observed for inhibitors of MK2 [59]. Inhibition of Chk1 and Wee1 in combination was particularly effective $[1,45,46]$ but faces the risk of unacceptable toxicities, which may be ameliorated by pretreatment with Mdm2 inhibitors. Furthermore, substances with predominant toxicity to cells in mitosis are no longer limited to taxanes. Rather, tubulin stabilizers like epothilones as well as signaling inhibitors that preclude a smooth transition through mitosis, e. g. inhibitors of aurora or polo-like kinases [60], also represent suitable candidates for combination with $\mathrm{Mdm} 2$ antagonists, to limit their toxicities to normal cells. All these approaches would take advantage of the most commonly encountered genetic difference between human malignancies and non-transformed cells, i. e. a mutation in TP53, to tailor therapeutic strategies specifically towards cancer cells and away from other cells in a patient's body. In this way, it is expected that therapies will not only become more tolerable to a patient, but that the maximum doses of tumor-drugs can be augmented to increase therapeutic efficacy.

As a word of caution, the side effects of Mdm2 antagonists need to be considered in these strategies as well. At present, not much is known about how well such antagonists are tolerated, but a dozen phase I studies with Mdm2 antagonists have been registered (clinicaltrials. gov). It is conceivable that Mdm2 inhibition may increase the death of those cells that are particularly sensitive towards p53 (an unwanted on-target effect). A recent study on mice with a global but inducible genetic ablation of Mdm2 revealed that such sensitive tissues not only involve the bone marrow and the gut, but also the kidney [61]. However, an important difference between this model and pharmacological antagonists is the transient nature of the latter. While the genetic ablation of $\mathrm{Mdm} 2$ is complete and permanent, pharmacological Mdm2-inhibitors can abrogate Mdm2 activity to an extent that can be adapted to the situation, and Mdm2 can quickly revert to normal p53 antagonism after discontinuing drug administration. The impact of Nutlin alone on the survival was only moderate in most cases of a panel of p53-proficient cell lines [62, 63]. Only in cells with high amplifications of the Mdm2 gene, cells appear to become addicted to this oncogene, rendering them exquisitely sensitive to Nutlin [62]. Since such amplifications are not present in normal cells, we expect that toxicities of Mdm2-antagonizing drugs will be manageable. The same is true for other reported effects of Nutlin, such as DNA breakage [64]or endoreduplication [65] in some cell lines. However, careful assessment of ongoing clinical trials involving Mdm2 antagonists will be required. This will then help to avoid unwanted toxicities by adapting the drug doses and schedules, and possibly by chemically modifying the drugs to reduce their impact on p53-sensitive normal tissues. Moreover, the combination of Mdm2 antagonists with Wee1 inhibitors will require evaluation in animal models before being taken to the clinics.

Taken together, our results suggest that p53 is an important determinant of how Weel inhibitors can be used in the clinics. On the one hand, p53 activation in tumor cells must be avoided to prevent negative drug interference with Wee1 inhibitors when a tumor carries wild type p53. On the other hand, however, in p53-mutant tumors, the administration of an Mdm2 antagonist appears as a highly promising opportunity to circumvent the toxicities of Weel inhibitors and many other drugs that act in a cell cycle specific manner.

\section{MATERIALS AND METHODS}

\section{Culturing of human cell lines}

U2OS (human osteosarcoma) and HCT116 (colorectal carcinoma) cells were cultured in DMEM and McCoy's, respectively, with 10\% FCS, 200 $\mu$ M L-glutamine and antibiotics - 50U/ml Penicillin and Streptomycin, and $10 \mu \mathrm{g} / \mathrm{ml}$ Ciprofloxacin (Bayer). In addition, medium for U2OS cells contained $20 \mu \mathrm{g} / \mathrm{ml}$ Tetracycline. All media and chemicals except Ciprofloxacin were from Invitrogen. MCF10A (non-transformed breast epithelial) cells were cultured in DMEM/F-12 with 5\% horse serum (Sigma $\mathrm{H} 1138$ ), $0.5 \mu \mathrm{g} / \mathrm{ml}$ hydrocortisone (Sigma H-0888), $0.1 \mu \mathrm{g} / \mathrm{ml}$ cholera toxin (Sigma C-8052), 20ng/ml Human EGF(Sigma E-9644), and 1:1000 Insulin (Sigma I-9278).

\section{Preparation of whole cell lysates}

Cells were seeded in 6-well plates $\left(1.6 \times 10^{5}\right.$ cells per well) for the drug treatment. Cell lysates were prepared on ice. The cells were scraped off into the medium and pelleted by centrifugation at $1500 \mathrm{xg}$ for $3 \mathrm{~min}$ at $4^{\circ} \mathrm{C}$, followed by one wash in PBS. The cells were resuspended in $100 \mu$ l RIPA lysis buffer $(1 \%$ Triton $\mathrm{X}, 1 \%$ desoxycholate, $0.1 \% \mathrm{SDS}, 150 \mathrm{mMNaCl}, 10 \mathrm{mM}$ EDTA, 20 mMTris- $\mathrm{HCl}$ pH 7.5, 100.000KIE Aprotinin) freshly supplemented with $2 \mathrm{M}$ urea, $1 \mathrm{mg} / \mathrm{ml}$ leupeptine/ aprotinine, $0.1 \mathrm{M}$ pepstatin A, $0.1 \mathrm{M}$ pefabloc. After 20 min of shaking at $4^{\circ} \mathrm{C}$, the lysates were centrifuged at $15,700 \mathrm{xg}$ for $10 \mathrm{~min}$. Bicinchoninic acid (BCA) assay was used to normalize the concentration of proteins in the supernatant. The samples were then boiled with Laemmli buffer, followed by SDS-PAGE.

\section{Transfection of human cells}

Using lipofectamine 2000, we carried out transient transfection of U2OS cells with siRNA to knock-down p53, and a corresponding control siRNA as a control. Lipofectamine and siRNA were dissolved separately 
in DMEM only (without FCS, or and antibiotics) and incubated at room temperature (RT) for $5 \mathrm{~min}$. They were then combined and incubated for another $20 \mathrm{~min}$ at RT. In one well of a 6-well plate, 280,000 cells were seeded in $1.6 \mathrm{~mL}$ DMEM with $10 \% \mathrm{FCS}$, and $400 \mu \mathrm{L}$ of the lipofectamine-siRNA mix was added drop-wise, followed by a 48 hrs incubation.

\section{Immunoblot analysis}

Blots on nitrocellulose or PVDF membranes were stained with the following antibodies. Phosphorylated Ser 139 H2AX (05-636, Millipore), PARP (9542, Cell Signaling Technology), beta-Actin (ab6276-100, Abcam), phospho-H3 Ser 10 (3377, Cell signaling), p53 (sc-126, Santa Cruz Biotech), p21 (OP64, Calbiochem). Secondary antibodies coupled to horseradish peroxidase H3 (ab1791, Abcam) (Jackson Immunoresearch) were used for chemiluminescent detection (Millipore).

\section{Immunofluorescence analysis}

For immunofluorescence microscopy, the automated microscope Pathway 855 (Becton Dickinson, Franklin Lakes, NJ, United States) was used to read fluorescence intensity in 96-well plates. For confocal microscopy, the LSM 510 laser scanning microscope (Carl Zeiss, Germany) was used.

The cells were fixed in $3.7 \%$ paraformaldehyde for $20 \mathrm{~min}$, followed by permeabilization with $0.5 \%$ Triton-X in PBS for 15 min and blocking for 15 min using blocking solution ( $3 \% \mathrm{BSA}$ in $\mathrm{PBS})$. The primary antibody to phospho-H2AX (05-636, Millipore), diluted in blocking solution, was added for $1 \mathrm{~h}$, followed by incubation with a secondary antibody (Alexa-Fluor 546) and Hoechst 33342 (Invitrogen) diluted in blocking solution for $45 \mathrm{~min}$.

For EdU staining, permeabilization was followed by exposure to Click- iTEdU reaction cocktail (C10351, Invitrogen) for $30 \mathrm{~min}$. The cell nuclei were counterstained with Hoechst 33342.

Images were captured and analyzed using the BD Pathway software, wherein the region of interest (ROI), in this case the cell nuclei, were defined by Hoechst stain, and the average intensity of the antibody-coupled fluorescence within each ROI was determined.

\section{Caspase activity assay}

Cells were seeded in 6-well plates (1.6 x 10 (to the power of 5) cells per well) and treated with drugs. 24 hrs post-treatment, the cells were harvested (inclusive of medium) and centrifuged at $1500 \mathrm{xg}$ for $5 \mathrm{~min}$ at $4^{\circ} \mathrm{C}$. The pelleted cells were resuspended in $250 \mu 1$ caspase lysis buffer (1M Tris- $\mathrm{HCl}, 2 \mathrm{mM} \mathrm{MgCl}, 150 \mathrm{mM} \mathrm{NaCl}, 10 \mathrm{mM}$
DTT, Roche complete mini protease-inhibitor mix). They were shock-frozen thrice in liquid nitrogen and centrifuged at $15,000 \mathrm{xg}$ for $15 \mathrm{~min}$ at $4^{\circ} \mathrm{C} .40 \mu \mathrm{l}$ of lysate per well in a 96-well plate was distributed in triplicates. $10 \mu \mathrm{l}$ of AcDEVD-AMC substrate (working concentration $25 \mu \mathrm{M}$ ) (ALX-260-031 Enzo) was added to each sample. Caspase activity was measured using a fluorometer (Synergy MX 267137 ) at excitation wavelength $380 \mathrm{~nm}$ and emission wavelength $460 \mathrm{~nm}$ every $10 \mathrm{~min}$ for $4 \mathrm{hrs}$ at $37^{\circ} \mathrm{C}$.

\section{Cell cycle analysis by flow cytometry}

Cells were seeded in 6-well plates and treated with the drugs. After fixation in ethanol, the cells were washed in wash solution $(0.05 \%$ Triton-X in PBS), followed by incubation in staining solution $(2 \% \mathrm{FCS}, 0.2 \%$ Triton- $\mathrm{X}$ in PBS) with phospho-H3 antibody (3377, Cell signaling) for $2 \mathrm{hrs}$ and then with secondary antibody (coupled to AlexaFluor 488) for one hour. Subsequently, the cells were resuspended in $0.5 \mathrm{mg} / \mathrm{ml}$ RNAse A solution andincubated for $30 \mathrm{~min}$ at $37^{\circ} \mathrm{C}$. Directly before measurement, propidium iodide (final concentration: $30 \mu \mathrm{g} / \mathrm{ml}$ ) was added. Samples were measured using the flow cytometer Guava PCA-96 Base System (Millipore).

\section{Cell proliferation assay}

Cells were seeded in 96-well plates or 24-well plates, treated after 18-24 hrs, and the confluency of the cells was measured using a Celigo cell cytometer (Cyntellect; labeled as Day0). After 24 hrs, the medium was replaced with fresh media; the confluency was determined again (Day1); subsequent measurements were made every $24 \mathrm{hrs}$ and media was changed every $48 \mathrm{hrs}$.

\section{Cell viability assay}

Cells were seeded in 96-well plates with white walls and bottom and treated after 18-24 hrs. Cells treated with DMSO in a concentration which responds to the highest concentration of the drugs added were used as a control. Remaining wells without cells were filled with medium in order to obtain a value for background luminescence. Each experiment was incubated for 72 hrs. For measuring the luminescence, the CellTiterGlo®Luminescence Cell Viability Assay (Promega) was used. CellTiter-Glo ${ }^{\circledR R}$ eagent was added in a $1: 1$ ratio to the cell culture medium in a well. The plate was placed on an orbital shaker for $10 \mathrm{~min}$ for induction of cell lysis. Subsequently, the luciferase signal was measured on a LuminometerDLReady ${ }^{\text {TM}}$ Centro LB 960 reader. 


\section{Statistical analysis}

Statistical significance was determined using the unpaired, two-tailed Student's T-test. Significance was assumed for $p$-values below 0.05 . Asterisks in figures indicate resulting $\mathrm{p}$-values as follows: $* \mathrm{p}<0.05, * * \mathrm{p}$ $<0.01, * * * \mathrm{p}<0.001$. n.s. $=$ not significant. $n$ in figure legends indicates the number of independent experiments.

\section{ACKNOWLEDGEMENTS}

We are grateful to Dr. Norman Erytch for help in flow cytometry. This work was supported by the Wilhelm Sander Stiftung, the German José Carreras Leukemia foundation, and the German Cancer Aid.P. S. was a PhD student of the Göttingen Graduate School for Neurosciences, Biophysics, and Molecular Biosciences (GGNB).

\section{CONFLICTS OF INTEREST}

The authors disclose no potential conflicts of interest.

\section{GRANTS}

Wilhelm Sander Stiftung, José Carreras Foundation, Else Kröner-Fresenius-Stiftung, Deutsche Krebshilfe, Erasmus Mundus Scholarship (Eurindia Program), GGNB (Göttinger Graduiertenschule für Neurowissenschaften, Biophysik und Molekulare Biowissenschaften).

\section{REFERENCES}

1. Aarts M, Sharpe R, Garcia-Murillas I, Gevensleben H, Hurd MS, Shumway SD, Toniatti C, Ashworth A and Turner NC. Forced mitotic entry of S-phase cells as a therapeutic strategy induced by inhibition of WEE1. Cancer discovery. 2012; 2:524-539.

2. Osman AA, Monroe MM, Ortega Alves MV, Patel AA, Katsonis P, Fitzgerald AL, Neskey DM, Frederick MJ, Woo SH, Caulin C, Hsu TK, McDonald TO, Kimmel $\mathrm{M}$, Meyn RE, Lichtarge $\mathrm{O}$ and Myers JN. Wee-1 kinase inhibition overcomes cisplatin resistance associated with high-risk TP53 mutations in head and neck cancer through mitotic arrest followed by senescence. Molecular cancer therapeutics. 2015; 14:608-619.

3. Do K, Doroshow JH and Kummar S. Wee1 kinase as a target for cancer therapy. Cell cycle. 2013; 12:3159-3164.

4. Stathis A and Oza A. Targeting Wee1-like protein kinase to treat cancer. Drug news \& perspectives. 2010; 23:425-429.

5. Do K, Wilsker D, Ji J, Zlott J, Freshwater T, Kinders RJ, Collins J, Chen AP, Doroshow JH and Kummar S. Phase I Study of Single-Agent AZD1775 (MK-1775), a
Wee1 Kinase Inhibitor, in Patients With Refractory Solid Tumors. Journal of clinical oncology : official journal of the American Society of Clinical Oncology. 2015.

6. Parker LL and Piwnica-Worms H. Inactivation of the p34cdc2-cyclin B complex by the human WEE1 tyrosine kinase. Science. 1992; 257:1955-1957.

7. Watanabe N, Broome M and Hunter T. Regulation of the human WEE1Hu CDK tyrosine 15-kinase during the cell cycle. The EMBO journal. 1995; 14:1878-1891.

8. Beck H, Nahse V, Larsen MS, Groth P, Clancy T, Lees M, Jorgensen M, Helleday T, Syljuasen RG and Sorensen CS. Regulators of cyclin-dependent kinases are crucial for maintaining genome integrity in $\mathrm{S}$ phase. The Journal of cell biology. 2010; 188:629-638.

9. Dobbelstein $\mathrm{M}$ and Sorensen CS. Exploiting replicative stress to treat cancer. Nature reviews Drug discovery. 2015.

10. Plunkett W, Huang P, Searcy CE and Gandhi V. Gemcitabine: preclinical pharmacology and mechanisms of action. Seminars in oncology. 1996; 23:3-15.

11. Dobbelstein M and Moll U. Targeting tumour-supportive cellular machineries in anticancer drug development. Nature reviews Drug discovery. 2014; 13:179-196.

12. Saini P, Li Y and Dobbelstein M. Wee1 is required to sustain ATR/Chk1 signaling upon replicative stress. Oncotarget. 2015.

13. Beck H, Nahse-Kumpf V, Larsen MS, O'Hanlon KA, Patzke S, Holmberg C, Mejlvang J, Groth A, Nielsen O, Syljuasen RG and Sorensen CS. Cyclin-dependent kinase suppression by WEE1 kinase protects the genome through control of replication initiation and nucleotide consumption. Molecular and cellular biology. 2012; 32:4226-4236.

14. Olivier M, Hollstein M and Hainaut P. TP53 mutations in human cancers: origins, consequences, and clinical use. Cold Spring Harbor perspectives in biology. 2010; 2:a001008.

15. Bieging KT, Mello SS and Attardi LD. Unravelling mechanisms of p53-mediated tumour suppression. Nature reviews Cancer. 2014; 14:359-370.

16. Wade M, Li YC and Wahl GM. MDM2, MDMX and p53 in oncogenesis and cancer therapy. Nature reviews Cancer. 2013; 13:83-96.

17. Vassilev LT, Vu BT, Graves B, Carvajal D, Podlaski F, Filipovic Z, Kong N, Kammlott U, Lukacs C, Klein $\mathrm{C}$, Fotouhi $\mathrm{N}$ and Liu EA. In vivo activation of the p53 pathway by small-molecule antagonists of MDM2. Science. 2004; 303:844-848.

18. Khoo KH, Verma CS and Lane DP. Drugging the p53 pathway: understanding the route to clinical efficacy. Nature reviews Drug discovery. 2014; 13:217-236.

19. Kranz D and Dobbelstein M. Nongenotoxic p53 activation protects cells against S-phase-specific chemotherapy. Cancer research. 2006; 66:10274-10280.

20. Carvajal D, Tovar C, Yang H, Vu BT, Heimbrook DC and Vassilev LT. Activation of p53 by MDM2 antagonists can 
protect proliferating cells from mitotic inhibitors. Cancer research. 2005; 65:1918-1924.

21. Apontes P, Leontieva OV, Demidenko ZN, Li F and Blagosklonny MV. Exploring long-term protection of normal human fibroblasts and epithelial cells from chemotherapy in cell culture. Oncotarget. 2011; 2(3):222233.

22. Blagosklonny MV, Robey $\mathrm{R}$, Bates $\mathrm{S}$ and Fojo $\mathrm{T}$. Pretreatment with DNA-damaging agents permits selective killing of checkpoint-deficient cells by microtubule-active drugs. The Journal of clinical investigation. 2000; 105:533539.

23. Blagosklonny MV and Pardee AB. Exploiting cancer cell cycling for selective protection of normal cells. Cancer research. 2001; 61:4301-4305.

24. Blagosklonny MV and Darzynkiewicz Z. Cyclotherapy: protection of normal cells and unshielding of cancer cells. Cell cycle. 2002; 1:375-382.

25. Hirai H, Iwasawa $Y$, Okada M, Arai T, Nishibata T, Kobayashi M, Kimura T, Kaneko N, Ohtani J, Yamanaka K, Itadani H, Takahashi-Suzuki I, Fukasawa K, Oki H, Nambu T, Jiang J, et al. Small-molecule inhibition of Wee1 kinase by MK-1775 selectively sensitizes p53-deficient tumor cells to DNA-damaging agents. Molecular cancer therapeutics. 2009; 8:2992-3000.

26. Indovina $\mathrm{P}$, Marcelli E, Di Marzo D, Casini N, Forte IM, Giorgi F, Alfano L, Pentimalli F and Giordano A. Abrogating G(2)/M checkpoint through WEE1 inhibition in combination with chemotherapy as a promising therapeutic approach for mesothelioma. Cancer biology \& therapy. 2014; 15:380-388.

27. Kreahling JM, Foroutan P, Reed D, Martinez G, Razabdouski T, Bui MM, Raghavan M, Letson D, Gillies RJ and Altiok S. Weel inhibition by MK-1775 leads to tumor inhibition and enhances efficacy of gemcitabine in human sarcomas. PloS one. 2013; 8:e57523.

28. Rajeshkumar NV, De Oliveira E, Ottenhof N, Watters J, Brooks D, Demuth T, Shumway SD, Mizuarai S, Hirai H, Maitra A and Hidalgo M. MK-1775, a potent Wee1 inhibitor, synergizes with gemcitabine to achieve tumor regressions, selectively in p53-deficient pancreatic cancer xenografts. Clinical cancer research : an official journal of the American Association for Cancer Research. 2011; 17:2799-2806.

29. Wang Y, Decker SJ and Sebolt-Leopold J. Knockdown of Chk1, Wee1 and Myt1 by RNA interference abrogates G2 checkpoint and induces apoptosis. Cancer biology \& therapy. 2004; 3:305-313.

30. Lazebnik YA, Kaufmann SH, Desnoyers S, Poirier GG and Earnshaw WC. Cleavage of poly(ADP-ribose) polymerase by a proteinase with properties like ICE. Nature. 1994; 371:346-347.

31. Rogakou EP, Pilch DR, Orr AH, Ivanova VS and Bonner WM. DNA double-stranded breaks induce histone H2AX phosphorylation on serine 139. The Journal of biological chemistry. 1998; 273:5858-5868.

32. Kopper F, Bierwirth C, Schon M, Kunze M, Elvers I, Kranz D, Saini P, Menon MB, Walter D, Sorensen CS, Gaestel M, Helleday T, Schon MP and Dobbelstein M. Damageinduced DNA replication stalling relies on MAPK-activated protein kinase 2 activity. Proceedings of the National Academy of Sciences of the United States of America. 2013; 110:16856-16861.

33. Bohlman S and Manfredi JJ. p53-independent effects of Mdm2. Sub-cellular biochemistry. 2014; 85:235-246.

34. el-Deiry WS, Tokino T, Velculescu VE, Levy DB, Parsons R, Trent JM, Lin D, Mercer WE, Kinzler KW and Vogelstein B. WAF1, a potential mediator of p53 tumor suppression. Cell. 1993; 75:817-825.

35. Gottifredi V, Shieh S, Taya Y and Prives C. p53 accumulates but is functionally impaired when DNA synthesis is blocked. Proceedings of the National Academy of Sciences of the United States of America. 2001; 98:10361041.

36. Bunz F, Dutriaux A, Lengauer C, Waldman T, Zhou S, Brown JP, Sedivy JM, Kinzler KW and Vogelstein B. Requirement for p53 and p21 to sustain G2 arrest after DNA damage. Science. 1998; 282(5393):1497-1501.

37. Kastan MB, Onyekwere O, Sidransky D, Vogelstein B and Craig RW. Participation of p53 protein in the cellular response to DNA damage. Cancer research. 1991; 51:63046311.

38. Prigent $\mathrm{C}$ and Dimitrov S. Phosphorylation of serine 10 in histone H3, what for? Journal of cell science. 2003; 116:3677-3685.

39. Charrier-Savournin FB, Chateau MT, Gire V, Sedivy J, Piette J and Dulic V. p21-Mediated nuclear retention of cyclin B1-Cdk1 in response to genotoxic stress. Molecular biology of the cell. 2004; 15:3965-3976.

40. Brown CJ, Lain S, Verma CS, Fersht AR and Lane DP. Awakening guardian angels: drugging the p53 pathway. Nature reviews Cancer. 2009; 9:862-873.

41. Van Linden AA, Baturin D, Ford JB, Fosmire SP, Gardner L, Korch C, Reigan P and Porter CC. Inhibition of Wee1 sensitizes cancer cells to antimetabolite chemotherapeutics in vitro and in vivo, independent of p53 functionality. Molecular cancer therapeutics. 2013; 12:2675-2684.

42. Guertin AD, Li J, Liu Y, Hurd MS, Schuller AG, Long B, Hirsch HA, Feldman I, Benita Y, Toniatti C, Zawel L, Fawell SE, Gilliland DG and Shumway SD. Preclinical evaluation of the WEE1 inhibitor MK-1775 as single-agent anticancer therapy. Molecular cancer therapeutics. 2013; 12:1442-1452.

43. Hamilton DH, Huang B, Fernando RI, Tsang KY and Palena C. WEE1 inhibition alleviates resistance to immune attack of tumor cells undergoing epithelial-mesenchymal transition. Cancer research. 2014; 74:2510-2519.

44. Harris PS, Venkataraman S, Alimova I, Birks DK, 
Balakrishnan I, Cristiano B, Donson AM, Dubuc AM, Taylor MD, Foreman NK, Reigan $\mathrm{P}$ and Vibhakar R. Integrated genomic analysis identifies the mitotic checkpoint kinase WEE1 as a novel therapeutic target in medulloblastoma. Molecular cancer. 2014; 13:72.

45. Mak JP, Man WY, Ma HT and Poon RY. Pharmacological targeting the ATR-CHK1-WEE1 axis involves balancing cell growth stimulation and apoptosis. Oncotarget. 2014; 5:10546-10557.

46. Wang G, Niu X, Zhang W, Caldwell JT, Edwards H, Chen W, Taub JW, Zhao L and Ge Y. Synergistic antitumor interactions between MK-1775 and panobinostat in preclinical models of pancreatic cancer. Cancer letters. 2015; 356:656-668.

47. Weisberg E, Nonami A, Chen Z, Liu F, Zhang J, Sattler M, Nelson E, Cowens K, Christie AL, Mitsiades C, Wong KK, Liu Q, Gray N and Griffin JD. Identification of Wee1 as a novel therapeutic target for mutant RAS-driven acute leukemia and other malignancies. Leukemia. 2015; 29:2737.

48. Tokalov SV and Abolmaali ND. Protection of p53 wild type cells from taxol by nutlin-3 in the combined lung cancer treatment. BMC cancer. 2010; 10:57.

49. Sachweh MC, Drummond CJ, Higgins M, Campbell J and Lain S. Incompatible effects of p53 and HDAC inhibition on $\mathrm{p} 21$ expression and cell cycle progression. Cell death \& disease. 2013; 4:e533.

50. Zajkowicz A, Krzesniak M, Matuszczyk I, GlowalaKosinska M, Butkiewicz D and Rusin M. Nutlin-3a, an MDM2 antagonist and p53 activator, helps to preserve the replicative potential of cancer cells treated with a genotoxic dose of resveratrol. Molecular biology reports. 2013; 40:5013-5026.

51. Blagosklonny MV. Wt p53 impairs response to chemotherapy: make lemonade to spare normal cells. Oncotarget. 2012; 3:601-607.

52. Kranz D and Dobbelstein M. A killer promoting survival: p53 as a selective means to avoid side effects of chemotherapy. Cell cycle. 2012; 11:2053-2054.

53. Kranz D, Dohmesen C and Dobbelstein M. BRCA1 and Tip60 determine the cellular response to ultraviolet irradiation through distinct pathways. The Journal of cell biology. 2008; 182:197-213.

54. Rao B, Lain S and Thompson AM. p53-Based cyclotherapy: exploiting the 'guardian of the genome' to protect normal cells from cytotoxic therapy. British journal of cancer. 2013; 109:2954-2958.

55. van Leeuwen IM and Lain S. Pharmacological manipulation of the cell cycle and metabolism to protect normal tissues against conventional anticancer drugs. Oncotarget. 2011; 2:274-276.

56. van Leeuwen IM, Rao B, Sachweh $\mathrm{MC}$ and Lain S. An evaluation of small-molecule p53 activators as chemoprotectants ameliorating adverse effects of anticancer drugs in normal cells. Cell cycle. 2012; 11:1851-1861.

57. Garrett MD and Collins I. Anticancer therapy with checkpoint inhibitors: what, where and when? Trends in pharmacological sciences. 2011; 32:308-316.

58. Hall AB, Newsome D, Wang Y, Boucher DM, Eustace B, Gu Y, Hare B, Johnson MA, Milton S, Murphy CE, Takemoto D, Tolman C, Wood M, Charlton P, Charrier JD, Furey B, et al. Potentiation of tumor responses to DNA damaging therapy by the selective ATR inhibitor VX-970. Oncotarget. 2014; 5:5674-5685.

59. Morandell S, Reinhardt HC, Cannell IG, Kim JS, Ruf DM, Mitra T, Couvillon AD, Jacks T and Yaffe MB. A reversible gene-targeting strategy identifies synthetic lethal interactions between MK2 and p53 in the DNA damage response in vivo. Cell reports. 2013; 5:868-877.

60. Manchado E, Guillamot M and Malumbres M. Killing cells by targeting mitosis. Cell death and differentiation. 2012; 19:369-377.

61. Zhang Y, Xiong S, Li Q, Hu S, Tashakori M, Van Pelt C, You MJ, Pageon L and Lozano G. Tissue-specific and age-dependent effects of global Mdm2 loss. The Journal of pathology. 2014; 233:380-391.

62. Xia M, Knezevic D, Tovar C, Huang B, Heimbrook DC and Vassilev LT. Elevated MDM2 boosts the apoptotic activity of p53-MDM2 binding inhibitors by facilitating MDMX degradation. Cell cycle. 2008; 7:1604-1612.

63. Xia M, Knezevic D and Vassilev LT. p21 does not protect cancer cells from apoptosis induced by nongenotoxic p53 activation. Oncogene. 2011; 30:346-355.

64. Rigatti MJ, Verma R, Belinsky GS, Rosenberg DW and Giardina C. Pharmacological inhibition of Mdm2 triggers growth arrest and promotes DNA breakage in mouse colon tumors and human colon cancer cells. Molecular carcinogenesis. 2012; 51:363-378.

65. Shen H, Moran DM and Maki CG. Transient nutlin-3a treatment promotes endoreduplication and the generation of therapy-resistant tetraploid cells. Cancer research. 2008; 68:8260-8268. 


\section{Mdm2 inhibition confers protection of p53-proficient cells from the cytotoxic effects of Wee1 inhibitors}

\section{Supplementary Material}
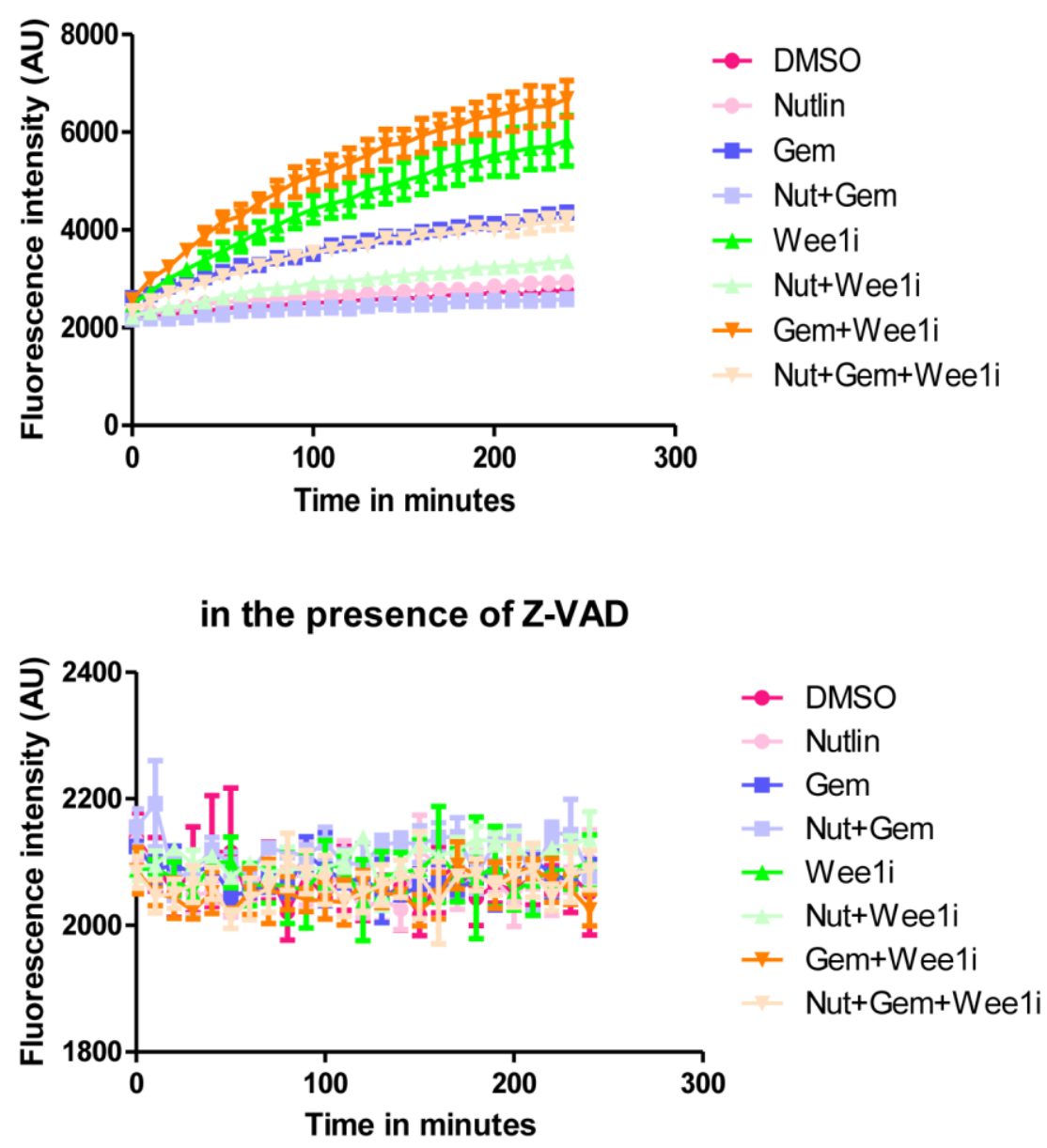

\section{Supplemental Figure 1. Caspase activity upon drug treatment}

U2OS cells were treated with $8 \mu \mathrm{M}$ Nutlin for $24 \mathrm{hrs}$, followed by treatment with $1 \mu \mathrm{M}$ Wee1

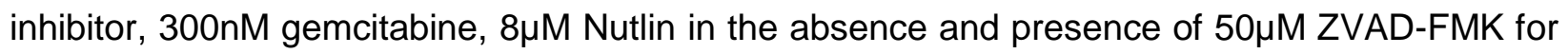
another 24 hrs. Cells were harvested and lysed for caspase activity assay. Fluorescence intensity measurements were obtained for each treatment. 


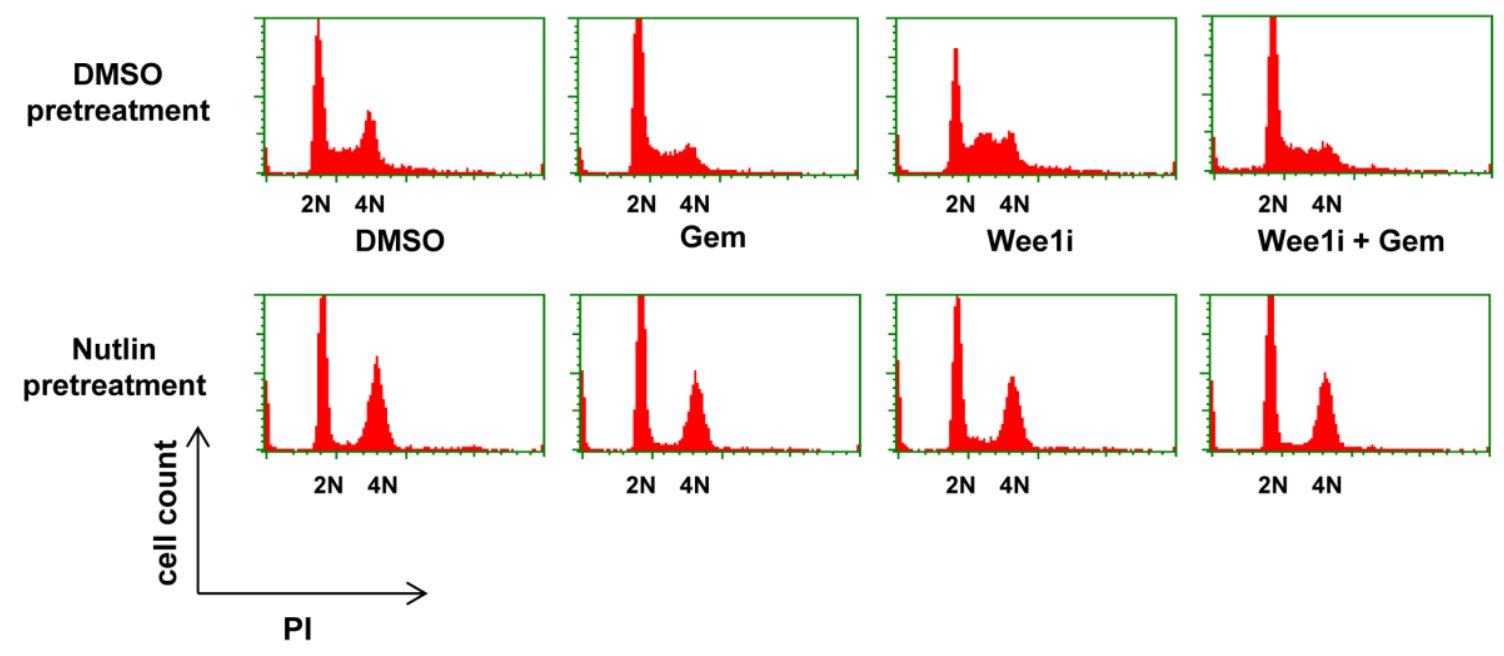

\section{Supplemental Figure 2. Nutlin reduces the amount of cells in S phase}

U2OS cells were treated as in Fig. 4A, followed by flow cytometry analysis of the DNA content. Histograms depict the relative number of cells found within a small window of DNA content, as determined by propidium iodide stain. The relative amount of cells with a DNA content between the G1 and G2/M peaks is reduced by Nutlin pretreatment, regardless of subsequent treatment with gemcitabine and/or Wee1i, arguing that Nutlin pretreatment largely precludes entry into $\mathrm{S}$ phase. 


\subsection{Publication II}

\section{Cooperation of Nutlin-3a and a WIP1 inhibitor to induce p53 activity}

\section{Citation}

Anusha Sriraman 1, Marija Radovanovic ${ }^{1}$, Magdalena Wienken 1, Zeynab Najafova 2, Yizhu Li 1,4, and Matthias Dobbelstein ${ }^{1,4}$

Cooperation of Nutlin-3a and a WIP1 inhibitor to induce p53 activity

Oncotarget. 2016 May 31;7(22):31623-38. doi: 10.18632/oncotarget.9302.

http://www.oncotarget.com/index.php?journal=oncotarget\&page=article\&op=view\&path[]=9302\&pubmed$\underline{\text { linkout }=1}$

Supplemental tables are available in the online version of the publication.

Own contribution: Carried out experiments and data analyses for Figures 1B, 2B (ii),3A (ii), 3B, 4-6, Supplemental figure 1-4, figure arrangement and contributions in writing and revising the manuscript. 


\title{
Cooperation of Nutlin-3a and a Wip1 inhibitor to induce p53 activity
}

\author{
Anusha Sriraman ${ }^{1}$, Marija Radovanovic ${ }^{1}$, Magdalena Wienken ${ }^{1}$, Zeynab Najafova ${ }^{2}$, \\ Yizhu Li ${ }^{1}$ and Matthias Dobbelstein ${ }^{1}$ \\ ${ }^{1}$ Institute of Molecular Oncology, Göttingen Center of Molecular Biosciences (GZMB), University Medical Center Göttingen, \\ Göttingen, Germany \\ ${ }^{2}$ Department of General, Visceral, and Pediatric Surgery, University Medical Center Göttingen, Göttingen, Germany \\ Correspondence to: Yizhu Li, email: yizhu.li@zentr.uni-goettingen.de \\ Matthias Dobbelstein, email: mdobbel@uni-goettingen.de
}

Keywords: Mdm2, Wip1/PPM1D, p53, kinase, phosphatase

Received: February 13,2016 Accepted: April 26, $2016 \quad$ Published: May 11, 2016

\section{ABSTRACT}

Targeting the Mdm2 oncoprotein by drugs has the potential of re-establishing p53 function and tumor suppression. However, Mdm2-antagonizing drug candidates, e. g. Nutlin-3a, often fail to abolish cancer cell growth sustainably. To overcome these limitations, we inhibited Mdm2 and simultaneously a second negative regulator of p53, the phosphatase Wip1/PPM1D. When combining Nutlin-3a with the Wip1 inhibitor GSK2830371 in the treatment of p53-proficient but not p53-deficient cells, we observed enhanced phosphorylation (Ser 15) and acetylation (Lys 382) of p53, increased expression of p53 target gene products, and synergistic inhibition of cell proliferation. Surprisingly, when testing the two compounds individually, largely distinct sets of genes were induced, as revealed by deep sequencing analysis of RNA. In contrast, the combination of both drugs led to an expression signature that largely comprised that of Nutlin-3a alone. Moreover, the combination of drugs, or the combination of Nutlin-3a with Wip1-depletion by siRNA, activated p53-responsive genes to a greater extent than either of the compounds alone. Simultaneous inhibition of Mdm2 and Wip1 enhanced cell senescence and G2/M accumulation. Taken together, the inhibition of Wip1 might fortify p53-mediated tumor suppression by Mdm2 antagonists.

\section{INTRODUCTION}

The tumor suppressor p53 is mutant in roughly $50 \%$ of all human malignancies, making it the most frequently mutated gene in human cancers. However, this notion also implies that another $50 \%$ of cancers still carry wild type p53 and nonetheless become malignant. In these cases, the tumor-suppressive activity of p53 is attenuated by regulatory mechanisms [1].

The best-characterized activity of p53 consists in transcriptional activation, through binding to its cognate promoter elements and recruiting transcription initiation factors as well as chromatin modifiers. This activity can be induced by cell stress signaling events, through a cascade of phosphorylations and acetylations. The p53-responsive genes and their products induce cell cycle arrest and/ or senescence (e. g. p21/CDKN1A), or apoptosis (e. g. Puma/BBC3). A third set of p53-inducible genes provides negative feedback on $\mathrm{p} 53$ activity, thereby attenuating the initial p53 response.

The expression of the negative regulator $\mathrm{Mdm} 2$ can be induced by $\mathrm{p} 53$. The $\mathrm{Mdm} 2$ protein binds to $\mathrm{p} 53$ and interferes with transactivation. Moreover, $\mathrm{Mdm} 2$ is an E3 ubiquitin ligase, leading to p53 ubiquitination and proteasomal destabilization. The Mdm2 gene is amplified in a considerable proportion of malignant tumors, most 
notably in sarcomas. In these cases, excessive amounts of Mdm2 largely abolish the tumor suppressive activity of p53. However, even when the copy number of the Mdm2 gene is normal, the p53-antagonizing activity of Mdm2 can still be exaggerated in tumors. The most wellestablished mechanisms for this consist in the silencing of a negative regulator of Mdm2, p14ARF (the second product of the gene CDKN2A), or in the enhanced expression of the heterodimerization partner of $\mathrm{Mdm} 2$, MdmX/Mdm4 [2].

Given the frequent silencing of p53 by the Mdm2 oncoprotein in tumors, it is conceivable that disrupting the interaction between the two proteins might re-establish p53-mediated tumor suppression [3]. Furthermore, the p53-binding structure on Mdm2, a hydrophobic pocket domain, can be occupied by a small molecule, making this one of the earliest example of "drugging" a protein-protein interaction. The most established compound to achieve this is Nutlin-3a [4], shortly referred to as Nutlin from here on. Nutlin binds to Mdm2, competing with p53. As a consequence, p53 becomes more active as a transcription factor and accumulates as a relatively stable protein, due to the lack of ubiquitination by Mdm2 [4].

Most cells respond to Nutlin largely by a reversible cell cycle arrest. Only a few cell lines - the majority of which containing heavily amplified Mdm2 - respond with apoptosis, thus rendering the drug efficient in cell killing. In the meantime, a number of drug candidates with similar structure and/or activity as Nutlin have been developed and have entered clinical trials $[3,5]$. A major concern in these trials consists in a possible lack of efficacy. Since many tumor cells merely arrest in response to Nutlin but resume proliferation once the drug is taken off, the clinical response might be transient at best. One way to get around this problem is to select tumors with a high frequency of Mdm2 amplifications, e. g. dedifferentiated liposarcomas $[6,7]$. Another way to fortify the efficacy of Nutlin and related drugs would be to combine them with additional compounds. This require targets that, when inactivated along with $\mathrm{Mdm} 2$, trigger an additive or even synergistic response.

Besides Mdm2, at least one additional p53responsive gene product antagonizes p53 activity. The gene Wip1/PPM1D, originally named after its plant homologue "wound-induced protein" [8], is induced by p53 [9]. Its product is a phosphatase that dephosphorylates a variety of proteins that are substrates to the kinase Ataxia Telangiectasia Mutated (ATM) [10] or related DNA damage-responsive pathways, e. g. ATM itself, Chk2 [11, 12], Chk1 [13] Histone 2AX [14-16], Mdm2 $[17]$ and p53 [13, 18]. The phosphorylation of p53 near its aminoterminus (e. g. at the residues Ser15 and Ser20) facilitates the association of acetyl transferases (e. g. p300 and CBP) with p53 and the subsequent acetylation of carboxyterminal residues [19]. This in turn enhances the binding of p53 to its target promoters. The phosphorylated aminoterminal domain then activates transcription, rendering p53 phosphorylation an essential trigger for gene activation [19]. When p53 induces Wip1, the dephosphorylation of p53 counteracts this activity and thus provides a negative feedback, similar to Mdm2. Like Mdm2, Wip1 can drive malignancy. This can happen through gene amplification, but also by truncating mutations near the 3' end of the coding region, destroying a negative regulatory domain of the Wip1 protein [20-24]. Most Wip1/PPM1D-amplified tumors harbor wild type p53, further arguing that the inactivation of p53 is at least one of the major activities of Wip1 [25].

Specific Wip1 inhibitors have recently been designed. In particular, the drug candidate GSK2830371 was shown to efficiently and specifically interfere with the phosphatase activity of Wip 1 through allosteric inhibition [26]. These inhibitors increase the phosphorylation of Wip1 substrates, including p53, and lead to a moderate increase in the expression of some p53 target genes. However, the cytotoxic efficacy of the inhibitors seemed moderate [26].

Of note, Mdm2 and Wip1 are acting by largely independent mechanisms. While Mdm2 triggers the degradation of p53 through the proteasome, Wip1 dephosphorylates its transactivating domain. This argues that inhibiting just one of these antagonists may be insufficient for full p53 activation. Instead, it would be more plausible to boost p53 activity by targeting both of these major antagonists simultaneously. In fact, the depletion or inactivation of both Wip1 and Mdm2 yields strong p53 activity [27-30].

Here we show that the combined inhibition of Mdm2 and Wip1 indeed abolishes cell proliferation in a synergistic and sustainable fashion. When applied together, Nutlin-3a and GSK2830371 induce strong accumulation of phosphorylated and acetylated p53. They also induce the accumulation of p53 target gene products in a p53-dependent fashion. Importantly, the genes induced by $\mathrm{Mdm} 2$ inhibition vs. Wip1 inhibition alone were largely distinct. Combining both drugs, however, primarily activated a large set of p53-responsive genes. Many of these genes were induced to a greater extent by the combination, rather than by Nutlin alone. Taken together, inhibiting Mdm2 and Wip1 simultaneously may represent a viable strategy to achieve strong p53 activation and permanent growth arrest, thereby diminishing or even preventing tumor progression. 
Wip1/PPM1D inhibitor GSK2830371 (Wip1i), and their combination. The cell lines chosen for this study were

Combined inhibition of Mdm2 and Wip1 synergistically diminishes cell proliferation

We tested the efficacy and sustainability of treatment with the Mdm2 inhibitor Nutlin-3a (Nutlin), the
MCF-7 (breast carcinoma) and U2OS (osteosarcoma), based on their known amplification (MCF-7) [23] or activating truncation (U2OS) [20] of Wip1. After treating the cells for 48 or $72 \mathrm{~h}$, the drugs were removed and the cells were continuously incubated for ten days. Cell proliferation was followed by automated translucent
MCF-7
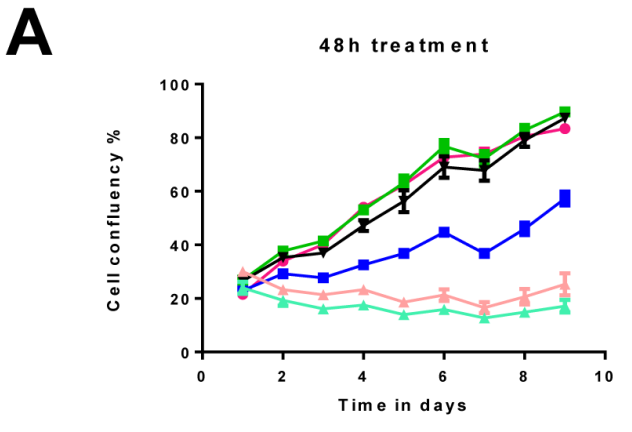

$72 \mathrm{~h}$ treatment

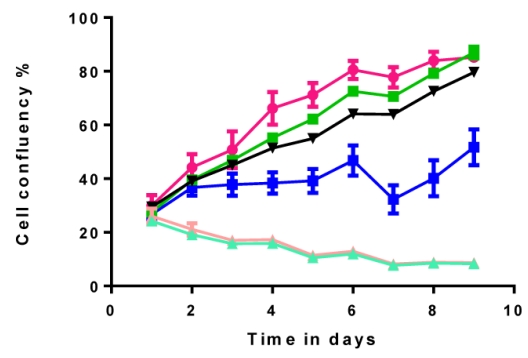

B

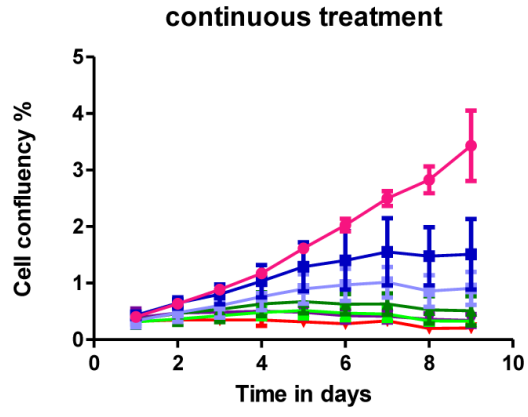

$\mathrm{Cl}-\mathbf{0 . 5 9}$ (synergistic) at day 6
U2OS
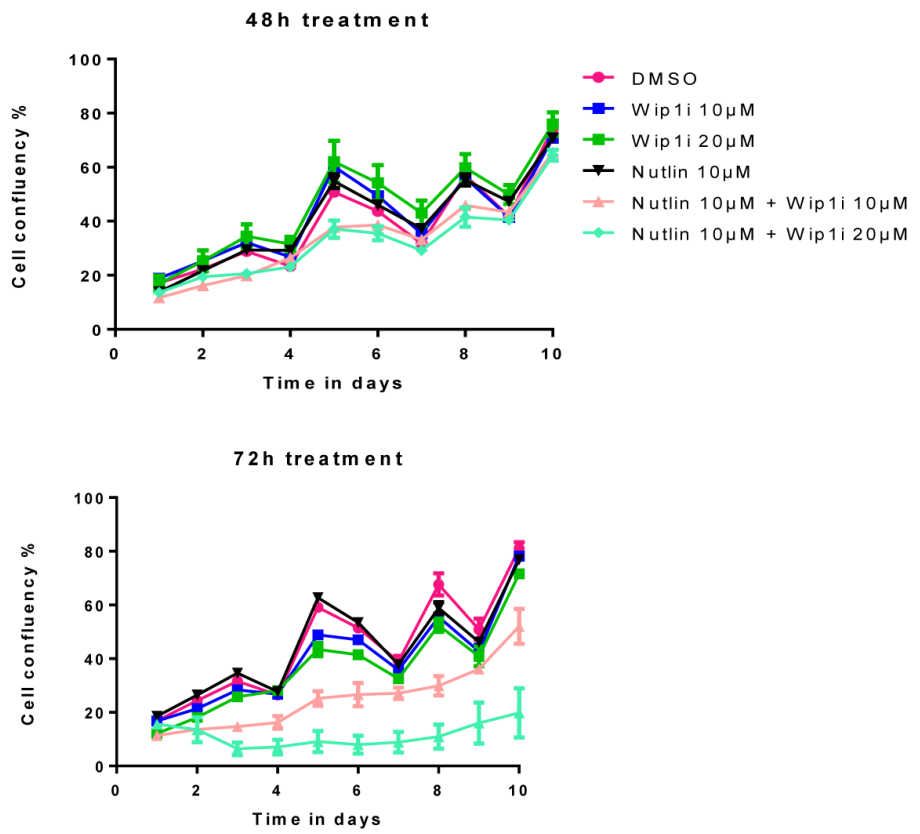

continuous treatment

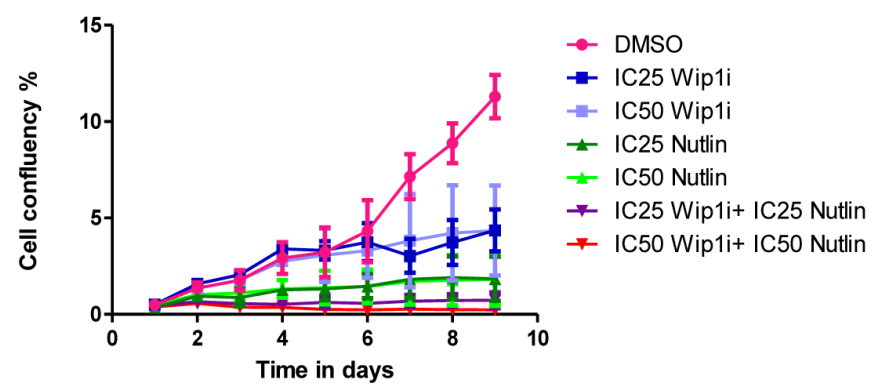

$\mathrm{Cl}-0.39$ (synergistic) at day 6

Figure 1: Synergistic impairment of cell proliferation by inhibition of Mdm2 and Wip1. A. Co-treatment by Wipli and Nutlin impedes cell growth in MCF-7 and U2OS cells. MCF-7 and U2OS cells were treated with Nutlin-3a (Nutlin), GSK2830371 (Wip1i) and its combinations at the indicated concentrations. After $48 \mathrm{~h}$ and $72 \mathrm{~h}$ of treatment, the drugs were removed and fresh medium was added. Cell confluency was measured every day using bright field microscopy (Celigo cell cytometer). Media was changed every 2-3 days, explaining the fluctuations in cell proliferation. B. Synergistic activity of Nutlin and Wip1i on MCF-7 and U2OS cells. MCF-7 and U2OS cells were treated with Nutlin and Wip1i at their IC25 and IC50, respectively, with continuous incubation. The cell confluency was measured daily as in A. Using the Chou-Talalay method [31], the combination index (CI) was calculated. At day 6, strong synergism was reflected by CI values way below 1 . 
microscopy. Both drugs were used at concentrations known to increase p53 levels or to enhance the phosphorylation of ATM substrates (Supplemental Figure S1). Nonetheless, neither of the drugs prevented cell proliferation over this period of time, although Wip1 inhibition did slow down the growth rate to some extent. In contrast, the combination of both inhibitors profoundly compromised the outgrowth of both cell lines and prevented confluency over the entire duration of the experiment, with the MCF-7 cells being particularly responsive (Figure 1A). Thus, the two drugs did cooperate to abolish cancer cell proliferation in a sustainable fashion.

To determine whether the two compounds act in a formally synergistic way, we first determined the drug concentrations that reduce cell proliferation by $25 \%$ or $50 \%$ (IC25 and IC50, respectively). The drugs were then continuously applied to the cells at IC25 and IC50, alone or in combination, and the reduction in proliferation was determined six days after drug removal (Figure 1B). This allowed us to calculate the combination index (CI) according to the algorithm by Chou and Talalay [31]. As a result, CIs far below 1 were obtained, indicating strong synergism between the drugs. Thus, inhibition of Mdm2 and Wip1 not only add up to impair cell growth, but they truly synergize to provide permanent growth arrest.
MCF-7

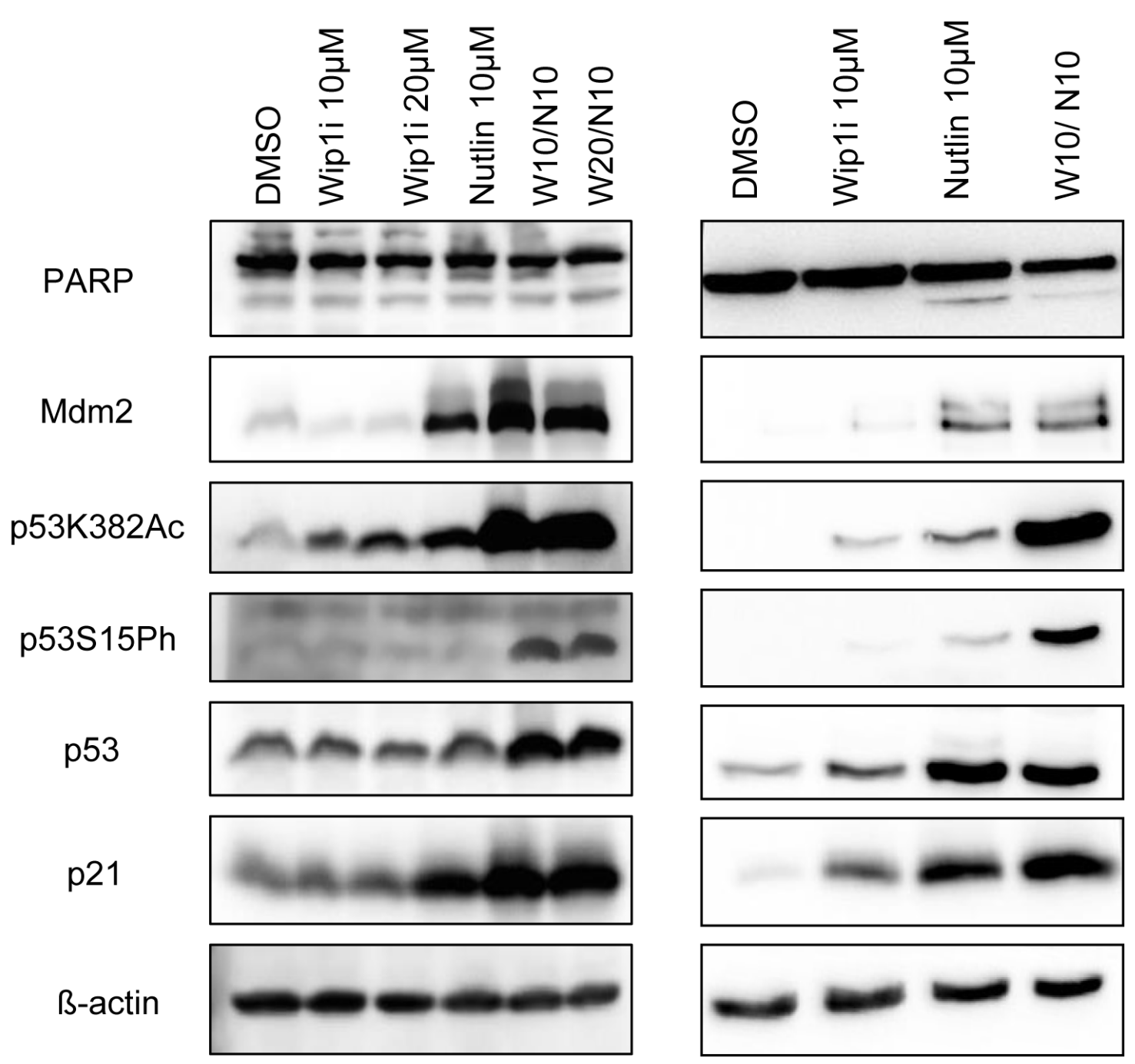

Figure 2: Accumulation of phosphorylated and acetylated p53 upon combined treatment. A. MCF-7 and U2OS cells were treated with Nutlin and Wip1i as indicated. After $24 \mathrm{~h}$, immunoblot analysis was performed to assay for the activation of p53 - phospho p53 and acetylated p53 - and its target gene products p21 and Mdm2. Actin staining served as the loading control. 
HCT116 p53-/- HCT116 p53+/+

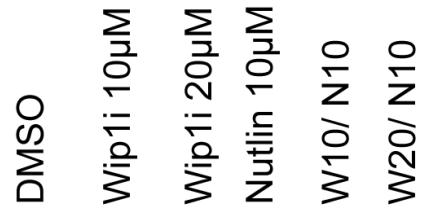
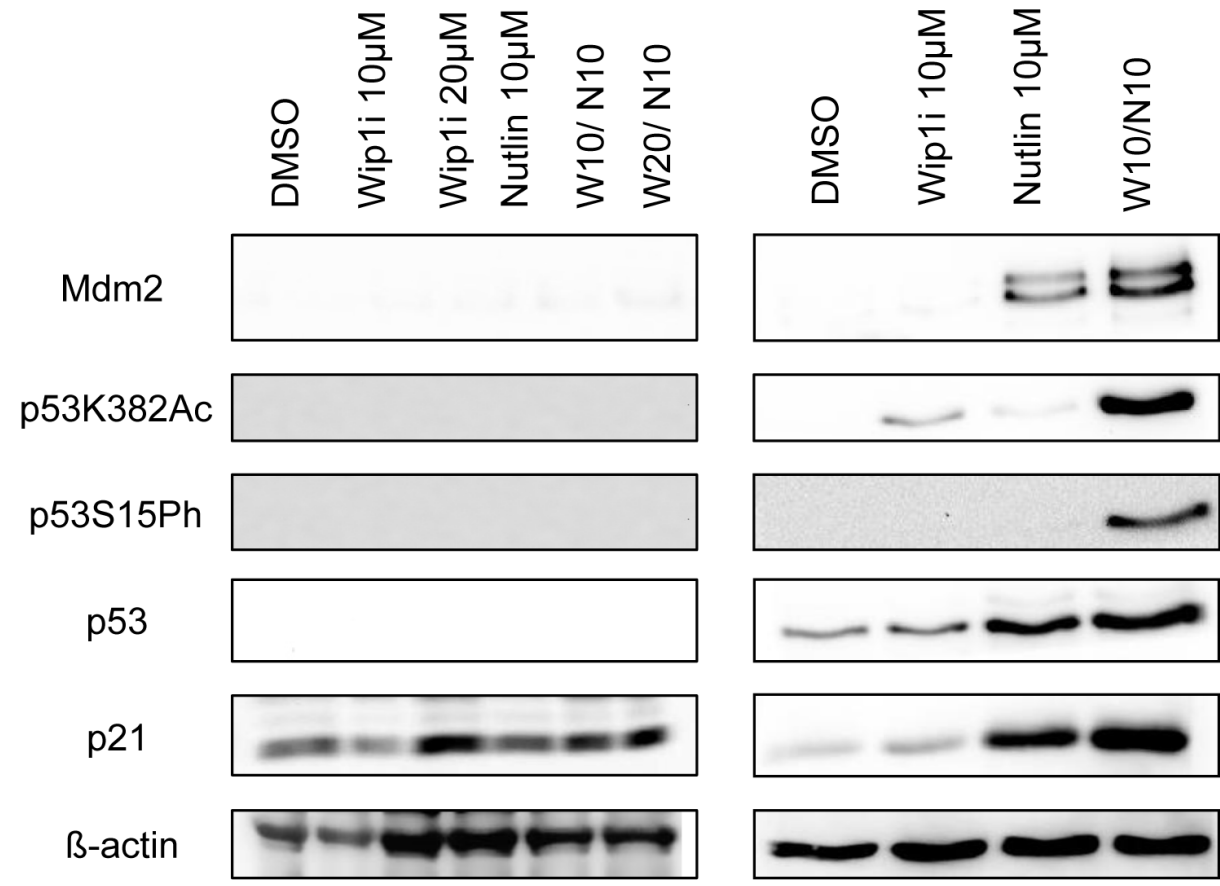

B
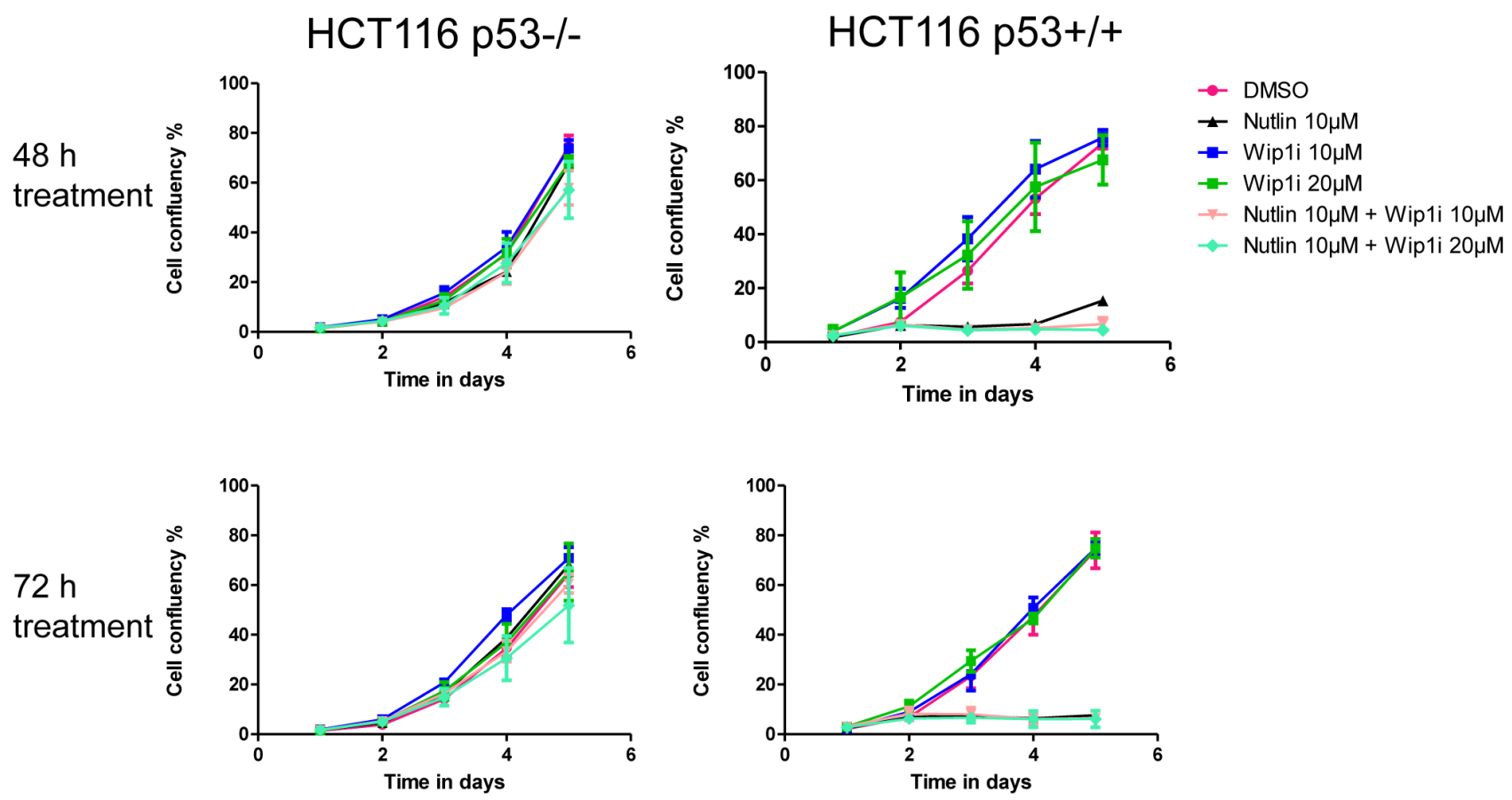

Figure 3: Activation of the p53 response upon co-treatment with Nutlin and Wip1i, dependent on p53 A. Co-treatment with Nutlin and Wipli leads to a p53 dependent response. An isogenic pair of HCT116 cells with and without a targeted deletion of TP53 [32] was treated with Nutlin and Wipli as indicated. After $24 \mathrm{~h}$, the cells were harvested to prepare protein lysates. Immunoblot analysis was performed to determine the amounts of p53 and its target gene products. B. Decrease in cell proliferation upon combined treatment is dependent on p53. Confluency for HCT116 cells with or without 533 was monitored for 5 days. The cells were treated with DMSO, Nutlin, Wipli and their combination for $48 \mathrm{~h}$ and $72 \mathrm{~h}$. Then, fresh medium was added and cell proliferation was monitored. 


\section{In combination with Wip1 inhibitor, Nutlin induces the accumulation of phosphorylated and acetylated p53}

To elucidate the mechanism of action underlying this drug synergism, we assessed the levels and modifications of p53, as well as the levels of p53inducible gene products by immunoblot analysis. Wip1 inhibition alone did not detectably affect the activity of p53 in MCF-7 cells, whereas in U2OS cells, it mildly increased the phosphorylation of p53 at serine 15 and its acetylation at lysine 382 . Correspondingly, the p53 target gene products $\mathrm{p} 21$ and $\mathrm{Mdm} 2$ were somewhat increased by Wip1 inhibition in U2OS cells but not in MCF-7 cells. Nutlin alone increased the levels of p53 and its target gene products in both cell lines. Importantly, when both drugs were combined, modified p53 strongly accumulated, along with p21 and Mdm2 (Figure 2). Of note, none of the treatments led to substantial increases in the cleavage of poly-(ADP ribose) polymerase (PARP), strongly suggesting that the observed reduction in cell numbers (cf. Figure 1) was not primarily a result of apoptosis. Along the same line, caspase activity was not induced by Nutlin or the combination of Nutlin with Wip1i (Supplemental Figure S2). In conclusion, Wip1 inhibition and Mdm2 inhibition cooperate to increase the phosphorylation and acetylation of $\mathrm{p} 53$, and to enhance the expression of the p53 target genes p21 and Mdm2.

\section{The induction of p21 and Mdm2, and growth inhibition by the drug combination, depend on p53}

Next, we tested whether the inhibitors of Wip1 and $\mathrm{Mdm} 2$ are increasing p21 and Mdm2 levels in a p53dependent fashion. To this end, we treated HCT116 cells (colon carcinoma, wild type $\mathrm{p} 53$, activating truncation of Wip1 [20]) or an HCT116-derived cell line with targeted disruption of the p53-encoding genes [32] with the same drugs, alone or in combination. As expected, we observed the accumulation of p21 and Mdm2 only in the p53proficient cells (Figure 3A), thereby largely excluding off-target effects or any other p53-independent effects of Wip1 and Mdm2 inhibition. In HCT116 cells again, the two drugs cooperated to induce the accumulation of phosphorylated and acetylated p53, and to enhance the expression of p53 target genes. Furthermore, we monitored the proliferation of HCT116 cells with and without p53 in response to the drugs. Reduced cell proliferation upon treatment with Nutlin and/or Wip1i was only observed in p53-proficient cells but not when p53 was deleted (Figure 3B). Thus, the efficacy of Nutlin and Wip1i strictly depends on p53. Finally, we tested whether the knockdown of Wip1 could mimic Wip1 inhibition.
We first depleted Wip1 from U2OS cells by siRNA and then monitored the expression of p53-responsive genes by quantitative RT-PCR, in the presence or absence of Nutlin. Indeed, the depletion of Wip1 increased the ability of Nutlin to augment the expression levels of p21, PUMA and PIG3 (Supplemental Figure S3A-S3D). Furthermore, when depleting Wip1 by siRNA, Nutlin compromised cell proliferation to a greater extent than upon control transfection (Supplemental Figure S3E). Thus, Wip1 depletion largely phenocopies Wip1 inhibition when combined with an Mdm2 inhibitor, strongly suggesting that the cooperation of Nutlin and Wipli actually depends on targeting Wip1.

\section{While each compound induces distinct gene sets, the combination largely enhances the gene signature of Nutlin}

To obtain a comprehensive overview on the genes induced by each of the drugs and their combination, we performed deep sequencing analysis of the RNA obtained from MCF-7 cells after treatment. As expected, large numbers of genes were found significantly regulated by each treatment (Figure 4A, Supplemental Table 1). Surprisingly, the overlap between genes that were upregulated by Nutlin and by Wip 1 inhibition was very limited, comprising only $7 \%$ of the Nutlin-responsive genes. Thus, Wip1 inhibition alone induces only a small subset of p53-responsive genes, including CDKN1A, FAS, and $\mathrm{Mdm} 2$. Otherwise, it appears to regulate genes by other means, e. g. through the phosphorylation of signaling factors that ultimately affect gene expression. Even more strikingly, however, the combination of Nutlin and Wipli elicited a gene expression signature that was far closer to Nutlin alone than to Wipli alone. The combination largely recapitulated the genes induced by Nutlin alone but enhanced their number by inducing additional genes, most of which had not been found inducible by either drug alone. In addition, gene induction was enhanced for a lot of Nutlin-responsive genes in the additional presence of Wip1i. Similarly, while Wip1i did not suppress the expression levels of any gene to a significant extent, Nutlin induced downregulation of p53-repressed genes. The mechanism of $\mathrm{p} 53$-mediated gene repression involves the CDK inhibitor p21 and a repressive complex, as described previously [33-36]. Importantly, co-treatment with Wip1i and Nutlin led to the repression of a broader set of genes which included virtually all the genes that had been repressed by Nutlin alone, again supporting the view that the combination broadens and intensifies p 53 activity (Figure 4B), and in agreement with previous studies on the role of Wip1 in the G2-M checkpoint [37].

Next, we sought to determine the induction of p53responsive genes in a more quantitative fashion, after 

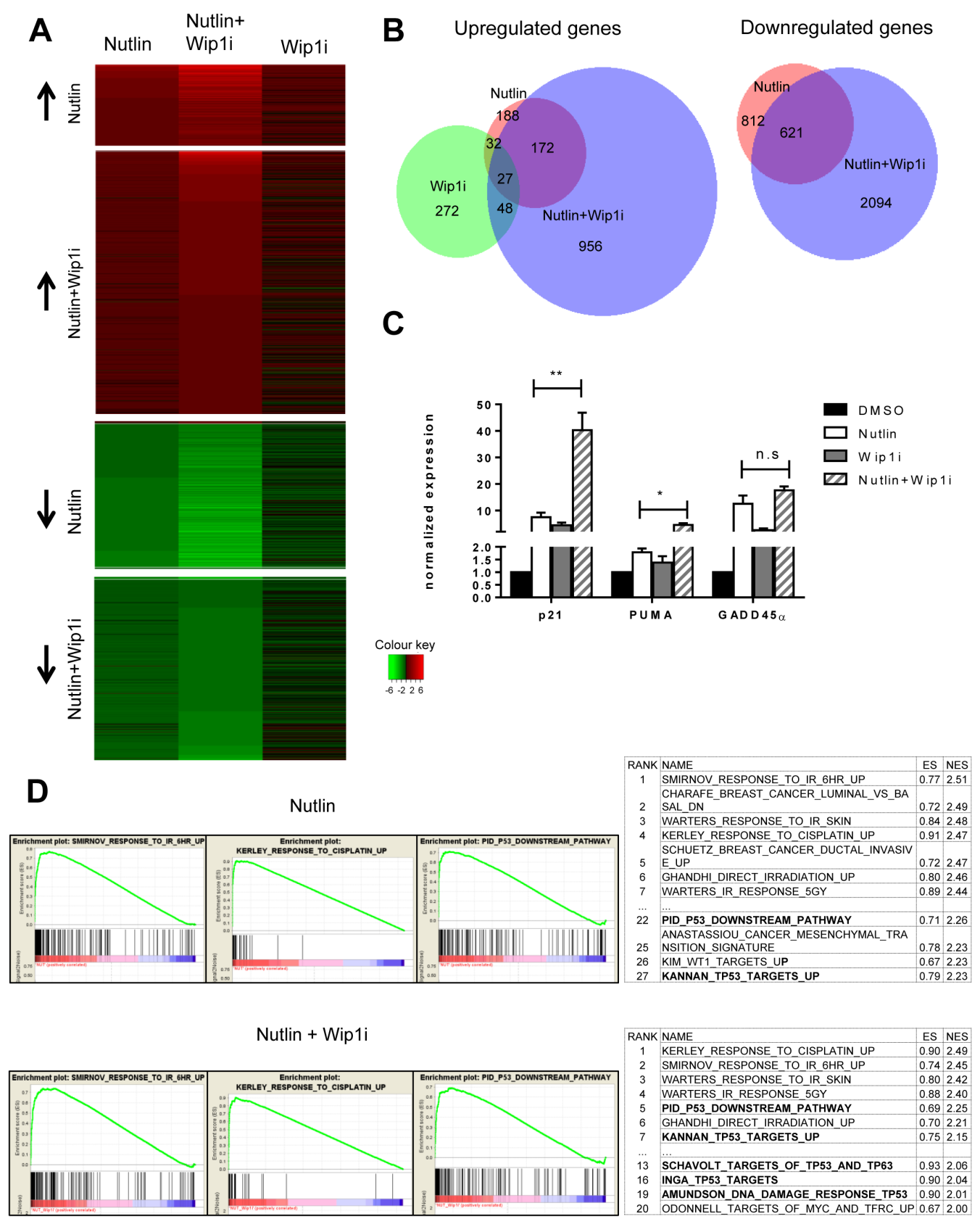

Figure 4: Broadened p53 response upon combined drug treatment, but not upon Wip1 inhibition alone. A. Heat map with $\log 2$-fold changes, resulting from mRNA-sequencing analysis of MCF-7 cells. Nutlin denotes cells treated with Nutlin at $10 \mu \mathrm{M}$ for $16 \mathrm{~h}$ compared to DMSO treatment. Wipli denotes cells treated with GSK 2830371 at $10 \mu \mathrm{M}$, and Nutlin+Wipli denotes cells treated with both drugs at the same concentrations, all for $16 \mathrm{~h}$ and compared to DMSO treatment. Only genes with Iog2-fold change $\geq 0.85$ and an adjusted p-value $\leq 0.05$ were included into the heat map. The number of differentially regulated genes under each condition were Nutlin - 474, Wip1i - 272, Nutlin+Wip1i - 1853. For single genes, cf. Supplemental Table 1. B. Venn diagrams depicting the significantly downregulated and upregulated genes in MCF-7 cells. Using the Bio-Venn software (www.cmbi.ru.nl/cdd/biovenn/index.php), the significantly upregulated and downregulated genes were plotted under each condition - Nutlin, Wip1i and Nutlin+Wip1i, each $v s$. DMSO. The Iog2-fold change was $\geq 0.85$ for Nutlin, Wip1i, and Nutlin+Wipli, and the adjusted p-value was $\leq 0.05$ for each sector. the corresponding numbers of genes are indicated. C. Enhancement of p53-induced mRNA synthesis by combined inhibition of Wip1 and Mdm2. MCF-7 cells were treated with the indicated combinations of inhibitors, followed by RNA preparation after $16 \mathrm{~h}$. Gene expression was quantified by real-time RT-PCR (mean \pm SEM, $n=3$ ). D. GO term analysis and functional annotation. Gene set enrichment analysis (GSEA) from C2 curated gene sets (provided by the Molecular Signatures Database (MSigDB) v5.0 [60,61]) was performed using variance stabilized RNA-Seq reads from Nutlin and Nutlin+Wipli treated samples. Selected enrichment plots from gene sets induced by Nutlin and Nutlin+Wipli are provided as examples. Ranking tables for induced gene sets are provided to demonstrate increased appearance of p53-responsive gene sets in the combination treatment compared to single treatment with Nutlin. 
treatment of MCF-7 cells with each or both of the two drugs. When analyzing mRNA levels by quantitative RT-PCR, we found that the combination of both drugs can induce p53-responsive genes up to 50-fold, whereas single drugs never exceeded 10-fold (Figure 4C). Thus, combining both drugs leads to a far more efficient induction of p53 activity than either compound alone. Of note, this degree of induction was greater than what was expected based on the immunoblot analysis shown in Figures 2 and 3. This may be due to the destabilization of p21 [38] and Mdm2 [39] proteins (but not mRNA) through the DNA damage response signaling elicited by Wip1 inhibition. Nonetheless, based on the plethora of additional p53-responsive genes induced, we propose that the boost in p53 activity through the combination of both inhibitors provides an explanation for the sustained growth arrest of p53-proficient cells observed in Figure 1.

Finally, gene set enrichment analysis (GSEA) revealed that "irradiation" and "p53 signaling pathway" were by far the most significantly enriched terms associated with the genes induced by the simultaneous treatment with Nutlin and Wip1i (Figure 4D; Supplemental Table 2). Remarkably, gene sets regarding p53 signaling pathways were induced to a much greater extent in the combination treatment in comparison to the Nutlin treatment alone, as indicated by GSEA term ranking.

\section{Nutlin and the combination of Nutlin with Wip1i preferentially induce genes with promoters that physically bind p53}

When comparing the induced genes with a database of promoters that had been found to associate with p53 in response to Nutlin ([40] Gene Omnibus database ID GSE47043), it turned out that the set of Nutlin-plusWip1i-inducible genes was highly enriched for promoter occupation by $\mathrm{p} 53$. This enrichment was found to a lesser degree with both of the single drugs, but not in the control-treated cells (Figure 5A and 5B). We furthermore identified p53 promoter binding on genes which were even more responsive towards the combination treatment than single Nutlin treatment. Indeed, we identified an especially strong p53 promoter correlation on these genes, indicating that their transactivation depended on both p53 activity and stability. In comparison, genes that were repressed by Nutlin and further repressed by the combinatorial treatment did not show comparable p53 binding, perhaps reflecting indirect regulation (Figure $5 \mathrm{C}$ and $5 \mathrm{D}$ ).

Taken together, these results indicate that the combination of the two inhibitors induces genes that have p53 associated with their promoters. This notion strongly suggests that the differential gene regulation by the two inhibitors is a direct consequence of the observed p53 activation.

\section{Combined inhibition of Wip1 and Mdm2 induces cell senescence}

Finally, we assessed possible mechanisms of how the combination of Nutlin and Wip1i abolishes cell proliferation. Of note, we had not observed enhanced caspase activity or PARP cleavage (Figure 2 and Supplemental Figure 2), and apoptosis-related pathways were not among the top hits of our GSEA analysis (Figure 4D), arguing against the idea that apoptosis makes a major contribution to drug efficacy in this case. On the other hand, the cells no longer proliferated after combined treatment, even when the drugs had been removed for more than a week (Figure 1A). We therefore suspected that senescence and/or permanent cell cycle arrest was induced upon drug treatment. Senescence was initially described as a mechanism of normal cell aging, due to loss of telomeres. More recently, however, acute senescence was shown to confer the efficacy of chemotherapeutics in many cases [41]. Nutlin induces senescence in a variety of tumor cell lines, albeit to different degrees [42]. We tested whether Wip1i further enhances the amount of senescent cells upon Nutlin treatment. p53-proficient HCT116 cells display senescence-associated beta-galactosidase activity upon treatment with chemotherapeutics [43] and were now assessed as to their senescence response upon treatment with Nutlin and Wip1. Indeed, Nutlin induced senescence in a fraction of cells, but this was further enhanced by Wip1i, whereas the inhibition of Wip1 alone did not lead to a detectable senescence response. Actually, the drug combination exceeded the efficacy of the gemcitabine control in senescence induction (Figure 6A and 6B). Next, we analyzed the cell cycle profile upon treatment with Nutlin, Wip1i and their combination in U2OS cells as well as p53-proficient or p53-deficient HCT116 cells, using propidium iodide (PI) staining and flow cytometry. In p53-proficient cells, Nutlin alone or together with Wip1i reduced the number of cells in S-phase, corresponding to the capability of p53 to induce cell cycle arrest. However, the combination of Wipli and Nutlin also increased the amount of cells with a $4 \mathrm{n}$ DNA content, corresponding to G2 or M (Figure 6C). This accumulation was dependent on p53 and was still observed at $48 \mathrm{~h}$ after removing the drugs (Supplemental Figure S4A- S4D). In conclusion, the combined inhibition of $\mathrm{Mdm} 2$ and Wip1 not only induces senescence in a fraction of p53-proficient cells, but also induces a sustainable arrest in G2/M.

\section{DISCUSSION}

Reviving the tumor suppressive activity of p53 has long been attempted for cancer treatment. With the development of $\mathrm{Mdm} 2$ inhibitors, this strategy appeared feasible but with limited efficacy. Here we show that the simultaneous inhibition of an additional p53-antagonist, 


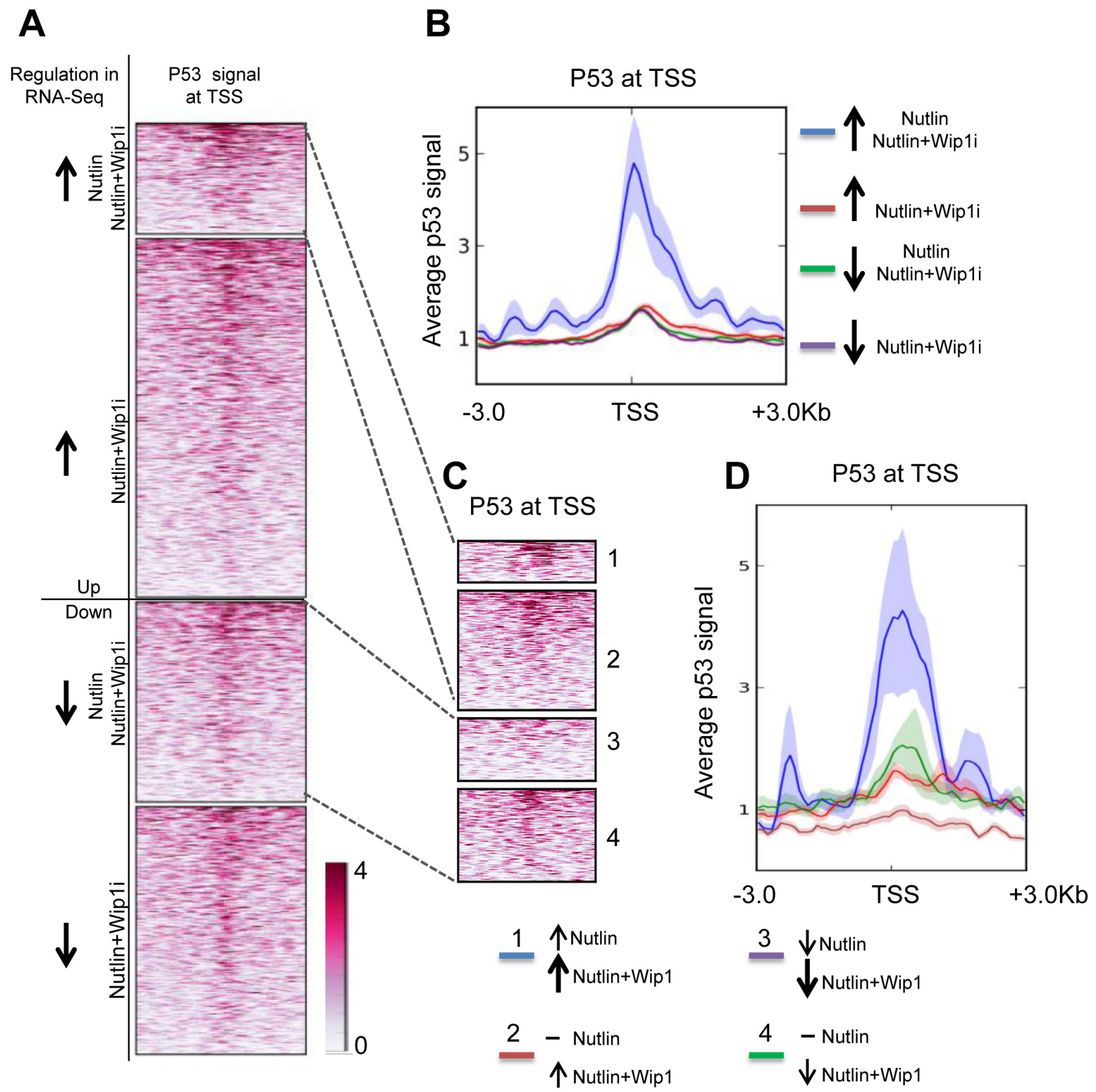

Figure 5: Induction of p53-bound genes by Nutlin and Wip1 inhibition. A. Heat map of p53 at transcription start sites of genes in MCF-7 cells after Nutlin treatment. Chip data on p53-promoter-associations are displayed, red color reflecting the degree of association with p53. Group 1 indicates those genes which were significantly upregulated by both Nutlin alone and Nutlin+Wip1i treatment in the RNA-Seq analysis from Figure 4. Group 2 indicates those genes that were significantly upregulated upon Nutlin+Wipli treatment but not by Nutlin alone. Group 3 indicates those genes that were significantly downregulated upon Nutlin and Nutlin+Wipli and group 4 indicates those genes that were downregulated upon Nutlin+Wipli treatment but not by Nutlin alone. B. Profiler image of p53 occupancy at transcription start sites of genes in MCF-7cells after Nutlin treatment. The profiler image (right) provides the average p53 signal obtained \pm $3 \mathrm{~kb}$ from the transcriptional start site for the genes at each of the above-mentioned conditions. ChIP-seq track data for Nutlin-3a-stimulated MCF-7 cells was obtained from p53 ChIP-Sequencing [40] and downloaded from the Gene Omibus database (ID GSE47043). C. Heat map of p53 on the TSSs of genes dependent on both p53 activity and stability. For better evaluation, we distinguished the genes from A, group 1 and 3, into two classifications, i. e. genes that were induced/downregulated further by the combined treatment in comparison to Nutlin alone (1 and 3), and the ones which were already induced/repressed by Nutlin alone to the maximum extent (2 and 4). D. Profiler image of p53 occupancy at TSSs of genes dependent on p53 activity and stability. The p53 promoter signal was aggregated along the TSSs of these genes as described in $\mathrm{C}$, and a profiler image is displayed on the right. 
HCT116 p53+/+
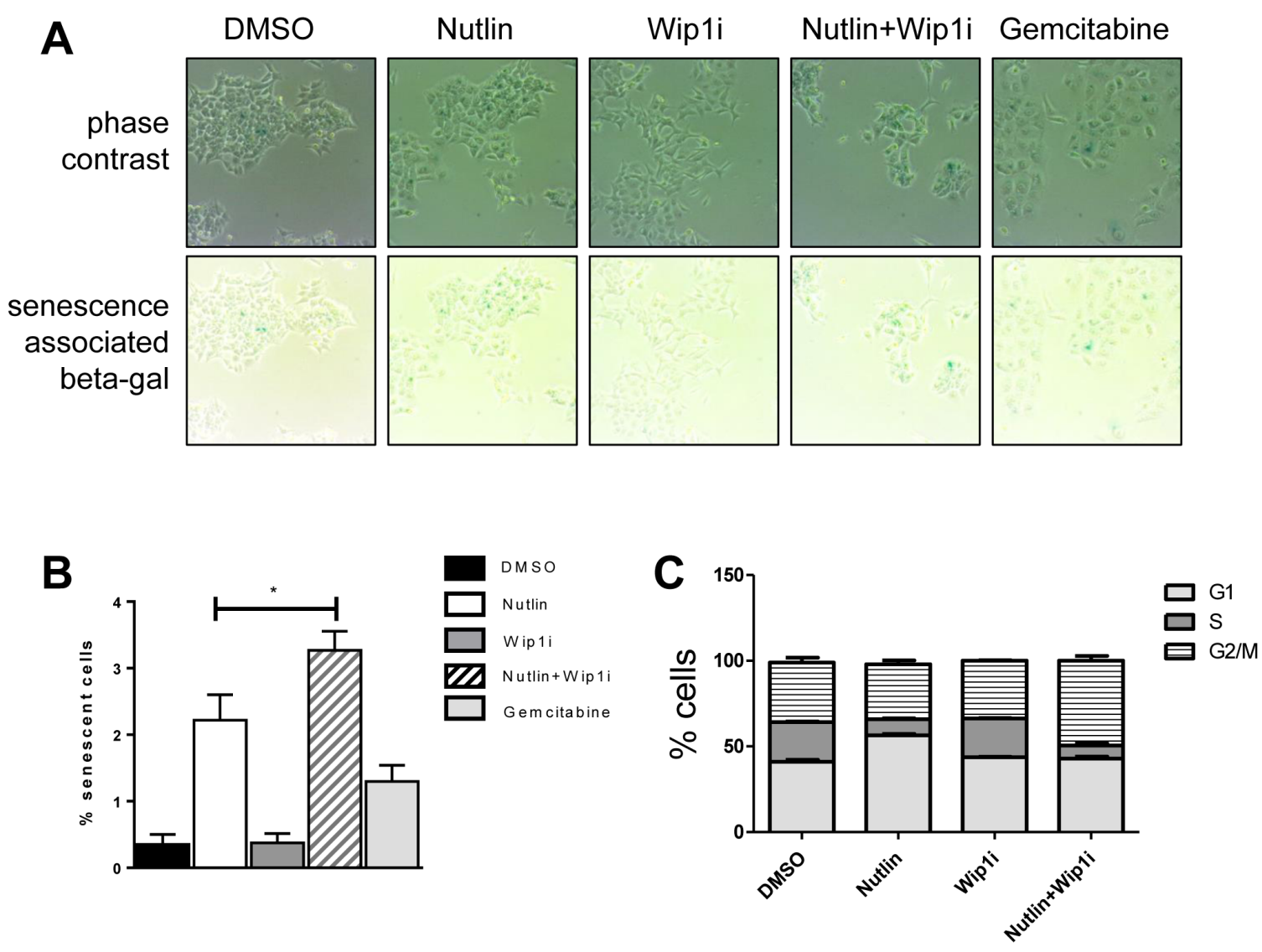

D

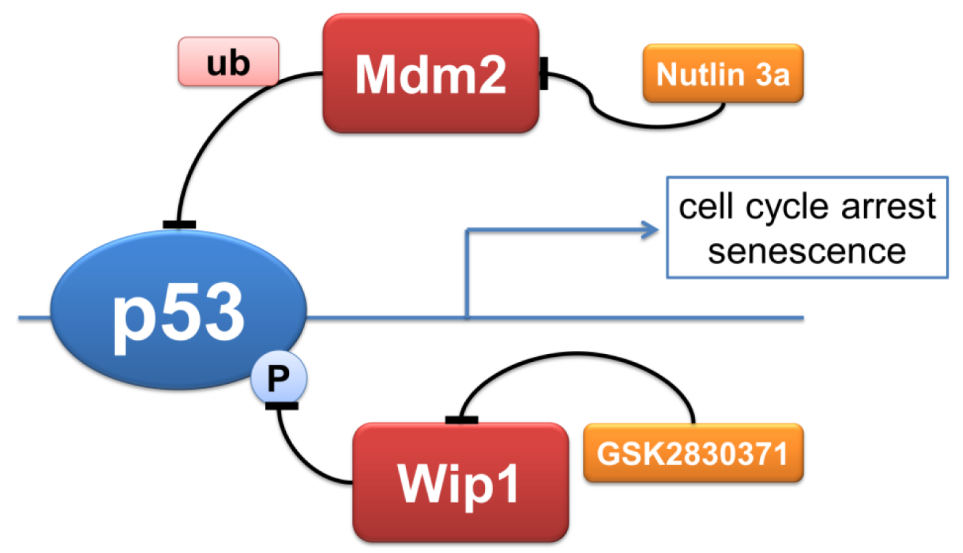

Figure 6: Enhanced cell senescence and G2/M accumulation by the drug combination. A. Senescence-associated betagalactosidase (SAB) induced by inhibition of Mdm2 and Wip1. HCT116 p53+/+ were seeded and treated with the indicated combinations of Nutlin and Wipli $(10 \mu \mathrm{M})$, or with $30 \mathrm{nM}$ Gemcitabine (positive control), for $48 \mathrm{~h}$. Senescent cells were stained using a senescenceassociated beta-galactosidase cell staining protocol (Cell Signaling \#9860). In the upper row, phase contrast images are provided to visualize all cells, whereas in the lower row, the same areas are shown without contrast, allowing the detection of the blue stain. B. Quantification of SAB accumulation. Using bright field microscopy, 10 images under each condition (A) were taken. Quantitative analysis was carried out using ImageJ, and the percentage of senescent cells to total cells was calculated. (mean $\pm \mathrm{SEM})$. C. Flow cytometry to determine the percentage of cells in each phase of the cell cycle. P53-proficient HCT116 cells were treated with the drugs for $24 \mathrm{~h}$ and harvested immediately for cell cycle analysis. Using propidium iodide, the percentage of cells in each phase was determined. For the full set of data, cf. Supplemental Figure S4. D. Cooperation of Nutlin and Wip1i to enhance p53 activity and cell growth arrest. p53 receives negative feedback upon induction of Mdm2 and Wip1. When both feedback regulators are targeted by drugs simultaneously, p53 activity is enhanced to a greater extent than with each drug alone. As a result, the cells undergo sustainable cell cycle arrest and/or senescence. 
Wip1/PPM1D, further enhances the activity of p53. Combined inhibitors have a considerably greater activity in conferring cell growth arrest, p53 accumulation, and the induction of p53-responsive genes. Thus, the combination of such drugs may provide a stronger anti-cancer treatment efficacy than the sole use of Mdm2-inhibitors (Figure 6D).

Our results agree with and expand recent reports on the use of the Mdm2 inhibitors RG7388 or Nutlin-3 and the Wip1 inhibitor GSK2830371 [29, 30]. In addition to their observations, we provide evidence that inhibition of both targets prevents the outgrowth of cells for ten days (Figure 1), the accumulation of acetylated p53 (Figures 2 and 3 ), and the finding that each of the inhibitors induces a largely distinct set of genes, whereas the combination of both promotes the enhanced expression of a gene set highly enriched of p53-induced and physically p53associated genes (Figures 4 and 5).

What makes tumor cells susceptible to the combined treatment? Firstly, a wildtype status for p53 is needed (Figure 3). Furthermore, hyperactive or amplified Wip1 might well render cells more responsive towards Wip1 inhibition. Indeed, MCF-7, U2OS and HCT116 cells all either overexpress Wip1 by means of gene amplification, or carry an activating truncation that removes a regulatory domain from the carboxyterminal portion of the protein $[20,29]$.

Of note, p53 activation may not always lead to tumor cell killing or permanent cell cycle arrest. On the contrary, we and others have previously observed that p53 activation by Nutlin can have a protective function on cells. Nutlin protects p53-proficient cells against nucleoside analogues and other inducers of replicative stress $[44,45]$, by temporarily preventing the entry to $S$ phase but perhaps also by regulation of BRCA1 expression [46]. This is generally true with regard to p53-inducing agents [47-50]. Moreover, we have recently shown that Nutlin also provides resistance of cells towards inhibitors of Wee1, a kinase that prevents premature mitosis [51]. Thus, when taking Nutlin and Wip1i to the clinics, care must be taken not to schedule their administration with drugs that require cell cycle progression for their efficacy. Besides nucleoside analogues, antagonists of the mitotic spindle, e. g. taxanes, were shown to be impaired in their efficacy by Nutlin [52], and we anticipate that the same will happen when trying to combine Wip1i, Nutlin, and spindle poisons.

On the other hand, some treatments might further synergize with inhibitors of Wip1 and Mdm2. In particular, it is conceivable that the induction of DNA damage, e. g. through ionizing irradiation, will trigger an ATM-driven response which is no longer counterbalanced by Wip1. This might then further augment the activity of p53 and other pro-apoptotic factors, thereby inducing cell death rather than the mere arrest of cell proliferation. A similar cooperative effect might also be achieved using BH3 mimetics [53] to increase the pro-apoptotic signal at the mitochondria, thereby tipping the balance towards apoptotic cell death.

Finally, interfering with the interaction of p53 and Mdm2 may not provide a block of all Mdm2-induced oncogenic activities. We have recently shown that $\mathrm{Mdm} 2$ associates with the polycomb repressor complex 2 (PRC2) and enhances its activities in suppressing gene expression, thus enabling a stem cell phenotype [54]. This activity of Mdm2 is not conferred by its p53-binding region and is thus not detectably affected by Nutlin. To achieve a broader inhibition of Mdm2 in most of its actions, inhibition of the RING finger domain and its E3 ubiquitin ligase activity might be more toxic to cancer cells. Such RING finger inhibitors were described [55], and their combination with Wip1i might yield a more thorough response of tumor cells. Finally, inhibitors that simultaneously interfere with the action of Mdm2 and its heterodimerization partner MdmX/Mdm4 were recently published [56], again showing a broader activity against tumor cells, and may thus warrant combination strategies with Wip1i.

Interfering with $\mathrm{Mdm} 2$ to restore the tumor suppressive activity of p53 appears like an attractive but insufficient strategy in most cases. However, the simultaneous interference with additional negative regulators and feedback loops raises the perspective of further boosting p53 and its ability to accumulate, activate transcription, abolish proliferation, and suppress tumor progression.

\section{MATERIALS AND METHODS}

\section{Cell culture and treatment}

U2OS (Osteosarcoma, p53 wild type) and MCF7 (breast carcinoma, p53 wild type) were maintained in Dulbecco's modified Eagle's medium (DMEM). HCT116 cells (colon cancer, p53 wild type or with a targeted deletion of $\mathrm{p} 53$ [32]) were maintained in McCoys 5A medium (1x). Cell culture media were supplemented with $10 \%$ fetal bovine serum (FBS) and antibiotics. Cells were maintained at $37^{\circ} \mathrm{C}$ in a humidified atmosphere with $5 \%$ $\mathrm{CO}_{2}$. For treatment of cells, Nutlin-3a (Sigma N6287), GSK2830371 (Active Biochem,CAS\#:1404456-53-6) stock solutions were prepared in DMSO and then diluted in pre-warmed medium and added to the cells for the indicated periods of time.

\section{Cell proliferation assay}

Cells were seeded in 24-well plates and the treatment was carried out as mentioned. The confluency of the cells was measured using a Celigo cell cytometer (Nexcelom; labeled as Day 0). After 24/48/72 h, the 
medium was replaced with fresh media; the confluency was determined again (Day 1); subsequent measurements were made every $24 \mathrm{~h}$ and media was changed every $48 \mathrm{~h}$.

\section{Transfection of human cells}

Transient transfection of U2OS cells with siRNAs to knock down PPM1D (Ambion silencer select s16288 and s16289, Thermo Fisher), and a corresponding control siRNA was carried out using Lipofectamine 2000. Lipofectamine and siRNA were dissolved separately in DMEM(without FCS, Glutamine, and antibiotics) and incubated at room temperature for $5 \mathrm{~min}$. Later, they were combined and incubated for another $20 \mathrm{~min}$. In one well of a 6-well plate, around 250,000 cells were seeded in $1.5 \mathrm{~mL}$ DMEM with supplements, and $500 \mu \mathrm{l}$ of the Lipofectamine-siRNA mix were added drop-wise. The cells were harvested $48 \mathrm{~h}$ after transfection.

\section{Immunoblot analysis}

Cells were harvested in protein lysis buffer (20 mM TRIS-HCl pH 7.5, $150 \mathrm{mM} \mathrm{NaCl}, 1$ mM Na EDTA, $1 \mathrm{mM}$ EGTA, $1 \mathrm{mM}$ beta-glycerophosphate, $2 \mathrm{M}$ Urea, Protease inhibitor Cocktail, Roche). After 10 min lysis on ice, samples were briefly sonicated to disrupt DNA-protein complexes. Total protein concentration was measured using a Pierce BCA Protein assay kit (Thermo Scientific Fisher). After boiling the samples in Laemmli buffer at $95^{\circ} \mathrm{C}$ for $5 \mathrm{~min}$, equal amounts of protein samples were separated by SDS-PAGE, transferred onto nitrocellulose, and visualized with the following antibodies: PARP1 (9542, Cell Signalling), $\gamma \mathrm{H} 2 \mathrm{AX}$ (S139) (9718, Cell Signalling), $\beta$-Actin (ab8227 abcam), p21 (2947, Cell signalling), Mdm2 (OP 46, Calbiochem), p53K382Ac (252S, Cell Signalling), p53phosphoS15 (9287S, Cell Signalling), p53 (DO-1 sc-126, Santa Cruz), phospho Chk2 (C13C1 2197, Cell Signalling).

\section{Determination of drug synergism}

Synergism between Nutlin and Wipli was determined in MCF-7 and U2OS cells. 2000 cells of MCF-7 and U2OS were seeded on 24-well plates. After $24 \mathrm{~h}$, they were treated with the IC 25 and IC 50 concentrations of the individual drugs, Nutlin-3a (Nutlin) and GSK2830371 (Wip1i). For MCF-7 cells, IC 25 and IC 50 for Nutlin was found to be $10 \mu \mathrm{M}$ and $20 \mu \mathrm{M}$, and IC 25 and IC 50 for Wipli was $20 \mu \mathrm{M}$ and $40 \mu \mathrm{M}$. For U2OS cells, IC 25 and IC 50 for Nutlin was found to be $10 \mu \mathrm{M}$ and $20 \mu \mathrm{M}$, and for Wip1i, IC 25 and IC 50 was $30 \mu \mathrm{M}$ and $40 \mu \mathrm{M}$. The confluency of cells was measured using a Celigo cell cytometer (Nexcelom). The cell confluency obtained for each drug concentration was normalized to treatment with the DMSO solvent alone. Using the software CompuSyn (www.combosyn.com), the combination index (CI) for the drug combinations was calculated (Chou, Talalay 2010). CI values $>1$ describe antagonistic or non-synergistic effects of two drugs, $\mathrm{CI}=$ 1 indicates additive effects and CI values $<1$ correspond to synergistic effects of combined drug treatment.

\section{RNA extraction, reverse transcription, and real time quantitative PCR}

Total RNA was extracted from cells using TRIzol ${ }^{\circledR}$ (Invitrogen). mRNA was reverse-transcribed using oligo$\mathrm{dT}$ and random hexameric primers, followed by qRT-PCR analysis using SYBR Green (Invitrogen). Gene expression levels were normalized to the mRNA encoding HPRT1, and the analysis was conducted using the $\Delta \Delta \mathrm{Ct}$ method. qRT-PCR primer sets were chosen as follows:

Primer sequences for gene expression studies in human cells

\begin{tabular}{|l|l|}
\hline Gene name & Primer sequence \\
\hline HPRT1 & $\begin{array}{l}\text { For- ATG CTG AGG ATT TGG AAA GG } \\
\text { Rev- TCA TCA CAT CTC GAG CAA } \\
\text { GAC }\end{array}$ \\
\hline P21 & $\begin{array}{l}\text { For- CCT GGC ACC TCA CCT GCT } \\
\text { CTG CTG } \\
\text { Rev- GCA GAA GAT GTA GAG CGG }\end{array}$ \\
\hline PUMA & $\begin{array}{l}\text { For- GCC AGA TTT GTG AGA CAA } \\
\text { GAG G } \\
\text { Rev- CAG GCA CCT AAT TGG GCT C }\end{array}$ \\
\hline GADD45 $\alpha$ & $\begin{array}{l}\text { For- TCA GCG CAC GAT CAC TGT C } \\
\text { Rev- CCA GCA GGC ACA ACA CCA C }\end{array}$ \\
\hline Wip1 & $\begin{array}{l}\text { For- CTG AAC CTG ACT GAC AGC CC } \\
\text { Rev- CTT GGC CAT GGA TCC TCC TC }\end{array}$ \\
\hline PIG3 & $\begin{array}{l}\text { For- GCT TCA AAT GGC AGA AAA GC } \\
\text { Rev- GTT CTT GTT GGC CTC CAT GT }\end{array}$ \\
\hline
\end{tabular}

RNA sequencing

For RNA-sequencing, the quality of total RNA was determined using the Bioanalyzer 2100 from Agilent. All samples analyzed exhibited a RNA Integrity Number $>$ 8. Library preparation was conducted using the TruSeq RNA LT SamplePrep Kit (Illumina), starting from 1000 ng of total RNA. Barcodes for sample preparation were used according to the indications given by the protocol. Accurate quantitation of cDNA libraries was performed with the QuantiFluor ${ }^{\mathrm{TM}} \mathrm{dsDNA}$ System (Promega). The size range of final cDNA libraries was determined applying the DNA 1000 chip on the Bioanalyzer 2100, (Agilent; 290-310 bp). cDNA libraries were amplified and sequenced via cBot and HiSeq 2000 (Illumina; SR, $1 \times 50 \mathrm{bp}, 6 \mathrm{~Gb} /$ sample ca. 30 million reads per sample). Sequence images were transformed with Illumina software BaseCaller to bcl files, which were demultiplexed to 
fastq files with CASAVA (version 1.8.2). Quality check was performed via FastQC (version 0.10.1, Babraham Bioinformatics). Fastq files were mapped to the human reference transcriptome (UCSC hg19) using Tophat (Galaxy Version 0.9) [57] Read counts for each sample and each gene were aggregated using a htseq-count [58]. DESeq2 (version 1.10.1) was used for measuring differential expression[59]. RNA library preparation and sequencing was done by the Transcriptome Analysis Laboratory (TAL, Göttingen).

Gene set enrichment analysis (GSEA) from C2 curated gene sets (provided by the Molecular Signatures Database (MSigDB) v5.0) was performed using variance stabilized normalized read counts. $[60,61]$. The threshold of significant enrichment $(\mathrm{q} \leq 0.25)$ was implied according to the GSEA standards (http://www.broadinstitute.org/ gsea/doc/GSEAUserGuideFrame.html).

\section{Correlation of RNA-Seq and p53 ChIP-seq data}

Raw data for p53 ChIP-Sequencing [40] were downloaded from the Gene Omibus database (ID GSE47043). The reads were mapped to the human reference genome (UCSC hg19) using Bowtie (version 1.0.0) [62]. Peak calling was done by Model-based Analysis of ChIP-Seq (version 1.4.2 [63]. Coverage was determined by normalizing the total number of mapped reads per hundred million. p53 enrichment was analyzed on the transcriptional start sites (TSSs) of genes that were upregulated in MCF-7 cells after Nutlin and after Nutlin+Wip1i treatment using deeptools functions [64] based on the Galaxy framework [65].

\section{Caspase activity assay}

Cells were seeded in 6-well plates and treated with drugs, At $24 \mathrm{~h}$ post-treatment, cells were harvested (inclusive of medium) and centrifuged at 1500xg for $5 \mathrm{~min}$ at $4^{\circ} \mathrm{C}$. The pelleted cells were resuspended in $250 \mu \mathrm{l}$ caspase lysis buffer $(1 \mathrm{M}$ Tris- $\mathrm{HCl}, 2 \mathrm{mM} \mathrm{MgCl}$, $150 \mathrm{mM} \mathrm{NaCl}, 10 \mathrm{mM}$ DTT, protease-inhibitor (Roche complete mini)). They were shock-frozen thrice in liquid nitrogen and centrifuged at $15,000 \mathrm{xg}$ for $15 \mathrm{~min}$ at $4^{\circ} \mathrm{C} .40 \mu \mathrm{l}$ of lysate was pipetted per well in a 96 -well plate in triplicates. $10 \mu \mathrm{l}$ of Ac-DEVD-AMC substrate (working concentration $25 \mu \mathrm{M}$ ) (ALX-260-031 Enzo) was added to each sample. Caspase activity was measured using a fluorometer (Synergy MX 267137) at excitation wavelength $380 \mathrm{~nm}$ and emission wavelength $460 \mathrm{~nm}$ every $10 \mathrm{~min}$ for $4 \mathrm{~h}$ at $37^{\circ} \mathrm{C}$.

\section{Cell cycle analysis by flow cytometry}

Cells were seeded in 6-well plates and treated with DMSO, Nutlin, Wip1i, and Nutlin+Wip1i. After fixation in ethanol, the cells were washed with $0.05 \%$ Triton-X in PBS. Subsequently, the cells were resuspended in $1 \mathrm{mg} / \mathrm{ml} \mathrm{RNAse}$ A solution in PBS and incubated for $30 \mathrm{~min}$ at $37^{\circ} \mathrm{C}$, and then with propidium iodide (final concentration: $30 \mu \mathrm{g} / \mathrm{ml}$ ). Flow cytometry was performed using the Guava PCA-96 Base System (Millipore), and the percentage of cells in each phase of the cell cycle was determined using the Guava Express Pro software.

\section{ACKNOWLEDGMENTS}

RNA seq data can be found using the GEO accession number - GSE80716. We thank the transcriptome analysis laboratory (TAL) of GZMB for performing RNA seq analyses. This work was supported by the Else Kröner Fresenius Stiftung, the Wilhelm Sander Stiftung, the Deutsche José Carreras Leukämie Stiftung, the Studienstiftung des deutschen Volkes and the Deutsche Krebshilfe.

\section{CONFLICTS OF INTEREST}

The authors declare no conflict of interest.

\section{Author contributions}

YL and MD conceived the project and designed experiments. MD drafted the manuscript. All authors revised the manuscript. AS, MR, and YL conducted the experiments. MW and ZN helped with the RNA Seq and further ChIP-Seq data analysis. YL and MD supervised the experiments. All authors read and approved the manuscript.

\section{REFERENCES}

1. Bieging KT, Mello SS and Attardi LD. Unravelling mechanisms of p53-mediated tumour suppression. Nature reviews Cancer. 2014; 14:359-370.

2. Wade M, Li YC and Wahl GM. MDM2, MDMX and p53 in oncogenesis and cancer therapy. Nature reviews Cancer. 2013; 13:83-96.

3. Khoo KH, Verma CS and Lane DP. Drugging the p53 pathway: understanding the route to clinical efficacy. Nature reviews Drug discovery. 2014; 13:217-236.

4. Vassilev LT, Vu BT, Graves B, Carvajal D, Podlaski F, Filipovic Z, Kong N, Kammlott U, Lukacs C, Klein $\mathrm{C}$, Fotouhi $\mathrm{N}$ and Liu EA. In vivo activation of the p53 pathway by small-molecule antagonists of MDM2. Science. 2004; 303:844-848. 
5. Patnaik A, Tolcher A, Beeram M, Nemunaitis J, Weiss GJ, Bhalla K, Agrawal M, Nichols G, Middleton S, Beryozkina A, Sarapa N, Peck R and Zhi J. Clinical pharmacology characterization of RG7112, an MDM2 antagonist, in patients with advanced solid tumors. Cancer Chemother Pharmacol. 2015; 76:587-595.

6. Bill KL, Garnett J, Meaux I, Ma X, Creighton CJ, Bolshakov S, Barriere C, Debussche L, Lazar AJ, Prudner BC, Casadei L, Braggio D, Lopez G, Zewdu A, Bid H, Lev D, et al. SAR405838: A Novel and Potent Inhibitor of the MDM2:p53 Axis for the Treatment of Dedifferentiated Liposarcoma. Clinical cancer research. 2015.

7. Kanojia D, Nagata Y, Garg M, Lee DH, Sato A, Yoshida K, Sato Y, Sanada M, Mayakonda A, Bartenhagen C, Klein HU, Doan NB, Said JW, Mohith S, Gunasekar S, Shiraishi $\mathrm{Y}$, et al. Genomic landscape of liposarcoma. Oncotarget. 2015; 6:42429-42444. doi: 10.18632/oncotarget.6464.

8. Rohrmeier T and Lehle L. WIP1, a wound-inducible gene from maize with homology to Bowman-Birk proteinase inhibitors. Plant Mol Biol. 1993; 22:783-792.

9. Fiscella M, Zhang H, Fan S, Sakaguchi K, Shen S, Mercer WE, Vande Woude GF, O'Connor PM and Appella E. Wip1, a novel human protein phosphatase that is induced in response to ionizing radiation in a p53-dependent manner. Proceedings of the National Academy of Sciences of the United States of America. 1997; 94:6048-6053.

10. Shreeram S, Demidov ON, Hee WK, Yamaguchi H, Onishi N, Kek C, Timofeev ON, Dudgeon C, Fornace AJ, Anderson CW, Minami Y, Appella E and Bulavin DV. Wip1 phosphatase modulates ATM-dependent signaling pathways. Mol Cell. 2006; 23:757-764.

11. Fujimoto H, Onishi N, Kato N, Takekawa M, Xu XZ, Kosugi A, Kondo T, Imamura M, Oishi I, Yoda A and Minami Y. Regulation of the antioncogenic Chk2 kinase by the oncogenic Wip1 phosphatase. Cell death and differentiation. 2006; 13:1170-1180.

12. Oliva-Trastoy M, Berthonaud V, Chevalier A, Ducrot C, Marsolier-Kergoat MC, Mann C and Leteurtre F. The Wip1 phosphatase (PPM1D) antagonizes activation of the Chk2 tumour suppressor kinase. Oncogene. 2007; 26:1449-1458.

13. Lu X, Nannenga $B$ and Donehower LA. PPM1D dephosphorylates Chk1 and p53 and abrogates cell cycle checkpoints. Genes Dev. 2005; 19:1162-1174.

14. Cha H, Lowe JM, Li H, Lee JS, Belova GI, Bulavin DV and Fornace AJ, Jr. Wip1 directly dephosphorylates gamma$\mathrm{H} 2 \mathrm{AX}$ and attenuates the DNA damage response. Cancer research. 2010; 70:4112-4122.

15. Macurek L, Lindqvist A, Voets O, Kool J, Vos HR and Medema RH. Wip1 phosphatase is associated with chromatin and dephosphorylates gammaH2AX to promote checkpoint inhibition. Oncogene. 2010; 29:2281-2291.

16. Moon SH, Nguyen TA, Darlington Y, Lu X and Donehower LA. Dephosphorylation of gamma-H2AX by WIP1: an important homeostatic regulatory event in DNA repair and cell cycle control. Cell cycle. 2010; 9:2092-2096.

17. Lu X, Ma O, Nguyen TA, Jones SN, Oren M and Donehower LA. The Wip1 Phosphatase acts as a gatekeeper in the p53-Mdm2 autoregulatory loop. Cancer Cell. 2007; 12:342-354.

18. Takekawa M, Adachi M, Nakahata A, Nakayama I, Itoh F, Tsukuda H, Taya Y and Imai K. p53-inducible wip1 phosphatase mediates a negative feedback regulation of p38 MAPK-p53 signaling in response to UV radiation. The EMBO journal. 2000; 19:6517-6526.

19. Bode AM and Dong Z. Post-translational modification of p53 in tumorigenesis. Nature reviews Cancer. 2004; 4:793805.

20. Kleiblova P, Shaltiel IA, Benada J, Sevcik J, Pechackova S, Pohlreich P, Voest EE, Dundr P, Bartek J, Kleibl Z, Medema RH and Macurek L. Gain-of-function mutations of PPM1D/Wip1 impair the p53-dependent G1 checkpoint. The Journal of cell biology. 2013; 201:511-521.

21. Ruark E, Snape K, Humburg P, Loveday C, Bajrami I, Brough R, Rodrigues DN, Renwick A, Seal S, Ramsay E, Duarte Sdel V, Rivas MA, Warren-Perry M, et al. Mosaic PPM1D mutations are associated with predisposition to breast and ovarian cancer. Nature. 2013; 493:406-410.

22. Lambros MB, Natrajan R, Geyer FC, Lopez-Garcia MA, Dedes KJ, Savage K, Lacroix-Triki M, Jones RL, Lord CJ, Linardopoulos S, Ashworth A and Reis-Filho JS. PPM1D gene amplification and overexpression in breast cancer: a qRT-PCR and chromogenic in situ hybridization study. Mod Pathol. 2010; 23:1334-1345.

23. Li J, Yang Y, Peng Y, Austin RJ, van Eyndhoven WG, Nguyen KC, Gabriele T, McCurrach ME, Marks JR, Hoey T, Lowe SW and Powers S. Oncogenic properties of PPM1D located within a breast cancer amplification epicenter at 17q23. Nat Genet. 2002; 31:133-134.

24. Zhang L, Chen LH, Wan H, Yang R, Wang Z, Feng J, Yang S, Jones S, Wang S, Zhou W, Zhu H, Killela PJ, Zhang J, Wu Z, Li G, Hao S, et al. Exome sequencing identifies somatic gain-of-function PPM1D mutations in brainstem gliomas. Nat Genet. 2014; 46:726-730.

25. Bulavin DV, Demidov ON, Saito S, Kauraniemi P, Phillips C, Amundson SA, Ambrosino C, Sauter G, Nebreda AR, Anderson CW, Kallioniemi A, Fornace AJ, Jr. and Appella E. Amplification of PPM1D in human tumors abrogates p53 tumor-suppressor activity. Nat Genet. 2002; 31:210-215.

26. Gilmartin AG, Faitg TH, Richter M, Groy A, Seefeld MA, Darcy MG, Peng X, Federowicz K, Yang J, Zhang SY, Minthorn E, Jaworski JP, Schaber M, et al. Allosteric Wip1 phosphatase inhibition through flap-subdomain interaction. Nat Chem Biol. 2014; 10:181-187.

27. Buss MC, Read TA, Schniederjan MJ, Gandhi K and Castellino RC. HDM2 promotes WIP1-mediated medulloblastoma growth. Neuro-oncology. 2012; 14:440458.

28. Choi M, Shi J, Jung SH, Chen X and Cho KH. Attractor 
landscape analysis reveals feedback loops in the p53 network that control the cellular response to DNA damage. Sci Signal. 2012; 5:ra83.

29. Esfandiari A, Hawthorne TA, Nakjang S and Lunec J. Chemical inhibition of wild-type p53 induced phosphatase 1 (WIP1/PPM1D) by GSK2830371 potentiates the sensitivity to MDM2 inhibitors in a p53-dependent manner. Molecular cancer therapeutics. 2016; 15:379-91.

30. Pechackova S, Burdova K, Benada J, Kleiblova P, Jenikova $\mathrm{G}$ and Macurek L. Inhibition of WIP1 phosphatase sensitizes breast cancer cells to genotoxic stress and to MDM2 antagonist nutlin-3. Oncotarget. 2016; doi: 10.18632/oncotarget.7363.

31. Chou TC. Drug combination studies and their synergy quantification using the Chou-Talalay method. Cancer research. 2010; 70:440-446.

32. Bunz F, Dutriaux A, Lengauer C, Waldman T, Zhou S, Brown JP, Sedivy JM, Kinzler KW and Vogelstein B. Requirement for p53 and p21 to sustain G2 arrest after DNA damage. Science. 1998; 282:1497-1501.

33. Dobbelstein M. Interchanging heads: p53 re-composes the DREAM/MMB complex to repress transcription. Cell cycle. $2013 ; 12: 11$.

34. Imbriano C, Gurtner A, Cocchiarella F, Di Agostino S, Basile V, Gostissa M, Dobbelstein M, Del Sal G, Piaggio G and Mantovani R. Direct p53 transcriptional repression: in vivo analysis of CCAAT-containing G2/M promoters. Molecular and cellular biology. 2005; 25:3737-3751.

35. Lohr K, Moritz C, Contente A and Dobbelstein M. p21/ CDKN1A mediates negative regulation of transcription by p53. The Journal of biological chemistry. 2003; 278:3250732516.

36. Quaas M, Muller GA and Engeland K. p53 can repress transcription of cell cycle genes through a p21(WAF1/ CIP1)-dependent switch from MMB to DREAM protein complex binding at CHR promoter elements. Cell cycle. 2012; 11:4661-4672.

37. Lindqvist A, de Bruijn M, Macurek L, Bras A, Mensinga A, Bruinsma W, Voets O, Kranenburg O and Medema RH. Wip1 confers G2 checkpoint recovery competence by counteracting $\mathrm{p} 53$-dependent transcriptional repression. The EMBO journal. 2009; 28:3196-3206.

38. Buscemi G, Ricci C, Zannini L, Fontanella E, Plevani P and Delia D. Bimodal regulation of p21(wafl) protein as function of DNA damage levels. Cell cycle. 2014; 13:29012912.

39. Malonia SK, Dutta P, Santra MK and Green MR. F-box protein FBXO31 directs degradation of MDM2 to facilitate p53-mediated growth arrest following genotoxic stress. Proceedings of the National Academy of Sciences of the United States of America. 2015; 112:8632-8637.

40. Verfaillie A, Imrichová H, Van de Sande B, Standaert L, Christiaens V, Hulselmans G, Herten K, Sanchez MN, Potier D and Svetlichnyy D. iRegulon: from a gene list to a gene regulatory network using large motif and track collections. PLoS Comput Biol. 2014; 10:e1003731.

41. Perez-Mancera PA, Young AR and Narita M. Inside and out: the activities of senescence in cancer. Nature reviews Cancer. 2014; 14:547-558.

42. Shen $\mathrm{H}$ and Maki CG. Persistent p21 expression after Nutlin-3a removal is associated with senescence-like arrest in $4 \mathrm{~N}$ cells. The Journal of biological chemistry. 2010; 285:23105-23114.

43. Braun CJ, Zhang X, Savelyeva I, Wolff S, Moll UM, Schepeler T, Orntoft TF, Andersen CL and Dobbelstein M. p53-Responsive micrornas 192 and 215 are capable of inducing cell cycle arrest. Cancer research. 2008; 68:1009410104.

44. Kranz D and Dobbelstein M. Nongenotoxic p53 activation protects cells against S-phase-specific chemotherapy. Cancer research. 2006; 66:10274-10280.

45. Apontes $\mathrm{P}$, Leontieva OV, Demidenko $\mathrm{ZN}, \mathrm{Li} \mathrm{F}$ and Blagosklonny MV. Exploring long-term protection of normal human fibroblasts and epithelial cells from chemotherapy in cell culture. Oncotarget. 2011; 2:222-233. doi: 10.18632/oncotarget.248.

46. Kranz D, Dohmesen C and Dobbelstein M. BRCA1 and Tip60 determine the cellular response to ultraviolet irradiation through distinct pathways. The Journal of cell biology. 2008; 182:197-213.

47. Blagosklonny MV. Wt p53 impairs response to chemotherapy: make lemonade to spare normal cells. Oncotarget. 2012; 3:601-607. doi: 10.18632/oncotarget.548.

48. Blagosklonny MV and Darzynkiewicz Z. Cyclotherapy: protection of normal cells and unshielding of cancer cells. Cell cycle. 2002; 1:375-382.

49. Blagosklonny MV and Pardee AB. Exploiting cancer cell cycling for selective protection of normal cells. Cancer research. 2001; 61:4301-4305.

50. Blagosklonny MV, Robey R, Bates $\mathrm{S}$ and Fojo $\mathrm{T}$. Pretreatment with DNA-damaging agents permits selective killing of checkpoint-deficient cells by microtubule-active drugs. The Journal of clinical investigation. 2000; 105:533539.

51. Li Y, Saini P, Sriraman A and Dobbelstein M. Mdm2 inhibition confers protection of $\mathrm{p} 53$-proficient cells from the cytotoxic effects of Wee1 inhibitors. Oncotarget. 2015; 6:32339-32352. doi: 10.18632/oncotarget.5891.

52. Carvajal D, Tovar C, Yang H, Vu BT, Heimbrook DC and Vassilev LT. Activation of p53 by MDM2 antagonists can protect proliferating cells from mitotic inhibitors. Cancer research. 2005; 65:1918-1924.

53. Delbridge AR and Strasser A. The BCL-2 protein family, BH3-mimetics and cancer therapy. Cell death and differentiation. 2015; 22:1071-1080.

54. Wienken M, Dickmanns A, Nemajerova A, Kramer D, Najafova Z, Weiss M, Karpiuk O, Kassem M, Zhang Y, Lozano G, Johnsen SA, Moll UM, Zhang X and 
Dobbelstein M. MDM2 Associates with Polycomb Repressor Complex 2 and Enhances Stemness-Promoting Chromatin Modifications Independent of p53. Mol Cell. 2016; 61:68-83.

55. Herman AG, Hayano M, Poyurovsky MV, Shimada K, Skouta R, Prives C and Stockwell BR. Discovery of Mdm2MdmX E3 ligase inhibitors using a cell-based ubiquitination assay. Cancer discovery. 2011; 1:312-325.

56. Chang YS, Graves B, Guerlavais V, Tovar C, Packman K, To KH, Olson KA, Kesavan K, Gangurde P, Mukherjee A, Baker T, Darlak K, Elkin C, et al. Stapled alpha-helical peptide drug development: a potent dual inhibitor of MDM2 and MDMX for p53-dependent cancer therapy. Proceedings of the National Academy of Sciences of the United States of America. 2013; 110:E3445-3454.

57. Kim D, Pertea G, Trapnell C, Pimentel H, Kelley R and Salzberg SL. TopHat2: accurate alignment of transcriptomes in the presence of insertions, deletions and gene fusions. Genome Biol. 2013; 14:R36.

58. Anders S, Pyl PT and Huber W. HTSeq-a Python framework to work with high-throughput sequencing data. Bioinformatics. 2014:btu638.

59. Love MI, Huber W and Anders S. Moderated estimation of fold change and dispersion for RNA-seq data with DESeq2. Genome Biol. 2014; 15:1-21.

60. Mootha VK, Lindgren CM, Eriksson K-F, Subramanian A, Sihag S, Lehar J, Puigserver P, Carlsson E, Ridderstråle $\mathrm{M}$ and Laurila E. PGC-1-responsive genes involved in oxidative phosphorylation are coordinately downregulated in human diabetes. Nature genetics. 2003; 34:267-273.
61. Subramanian A, Tamayo P, Mootha VK, Mukherjee S, Ebert BL, Gillette MA, Paulovich A, Pomeroy SL, Golub TR and Lander ES. Gene set enrichment analysis: a knowledge-based approach for interpreting genome-wide expression profiles. Proceedings of the National Academy of Sciences of the United States of America. 2005; 102:15545-15550.

62. Langmead B, Trapnell C, Pop M and Salzberg SL. Ultrafast and memory-efficient alignment of short DNA sequences to the human genome. Genome Biol. 2009; 10:R25.

63. Zhang Y, Liu T, Meyer CA, Eeckhoute J, Johnson DS, Bernstein BE, Nusbaum C, Myers RM, Brown M and Li W. Model-based analysis of ChIP-Seq (MACS). Genome Biol. 2008; 9:R137.

64. Ramírez F, Dündar F, Diehl S, Grüning BA and Manke T. deepTools: a flexible platform for exploring deep-sequencing data. Nucleic acids research. 2014; 42:W187-W191.

65. Blankenberg D, Von Kuster G, Coraor N, Ananda G, Lazarus R, Mangan M, Nekrutenko A and Taylor J. Galaxy: a web-based genome analysis tool for experimentalists. Current protocols in molecular biology / edited by Frederick M Ausubel [et al]. 2010; Chapter 19:Unit 1910 11-21.

66. Janicke RU, Sprengart ML, Wati MR and Porter AG. Caspase-3 is required for DNA fragmentation and morphological changes associated with apoptosis. The Journal of biological chemistry. 1998; 273:9357-9360. 


\section{Cooperation of Nutlin-3a and a Wip1 inhibitor to induce p53 activity}

\section{Supplementary Material}

\section{U2OS}

Wip1i

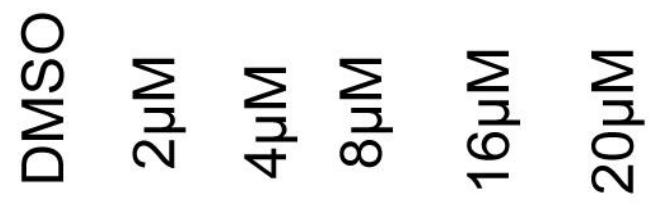

PARP

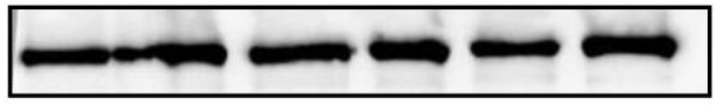

p53S15Ph

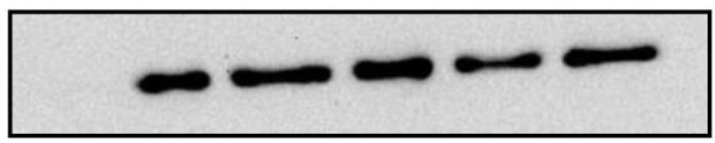

p53

Y-H2AX
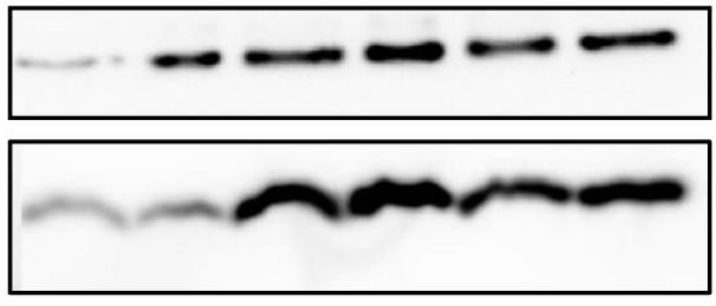

pChk2

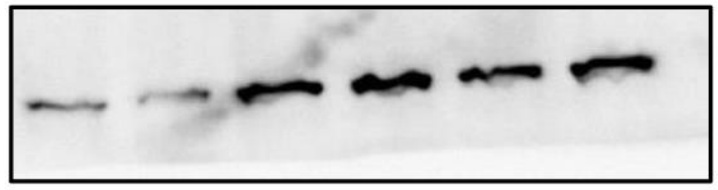

ß-actin

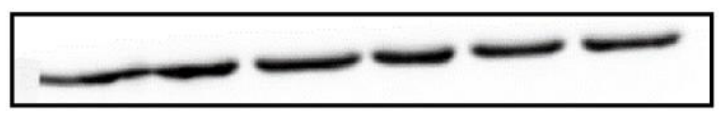

SUPPLEMENTAL FIGURE 1. Wip1i titration in U2OS cells. Related to Fig. 1

U2OS cells were treated with varying concentrations of Wip1i GSK 2830371. After $24 \mathrm{~h}$, the cells were harvested, followed by immunoblot analysis to detect the DNA damage-induced kinase substrates p53, phospho-p53, phospho-H2AX, and phospho-Chk2. PARP staining revealed the absence of detectable caspase activity. Actin staining served as the loading control. 


\section{U2OS}

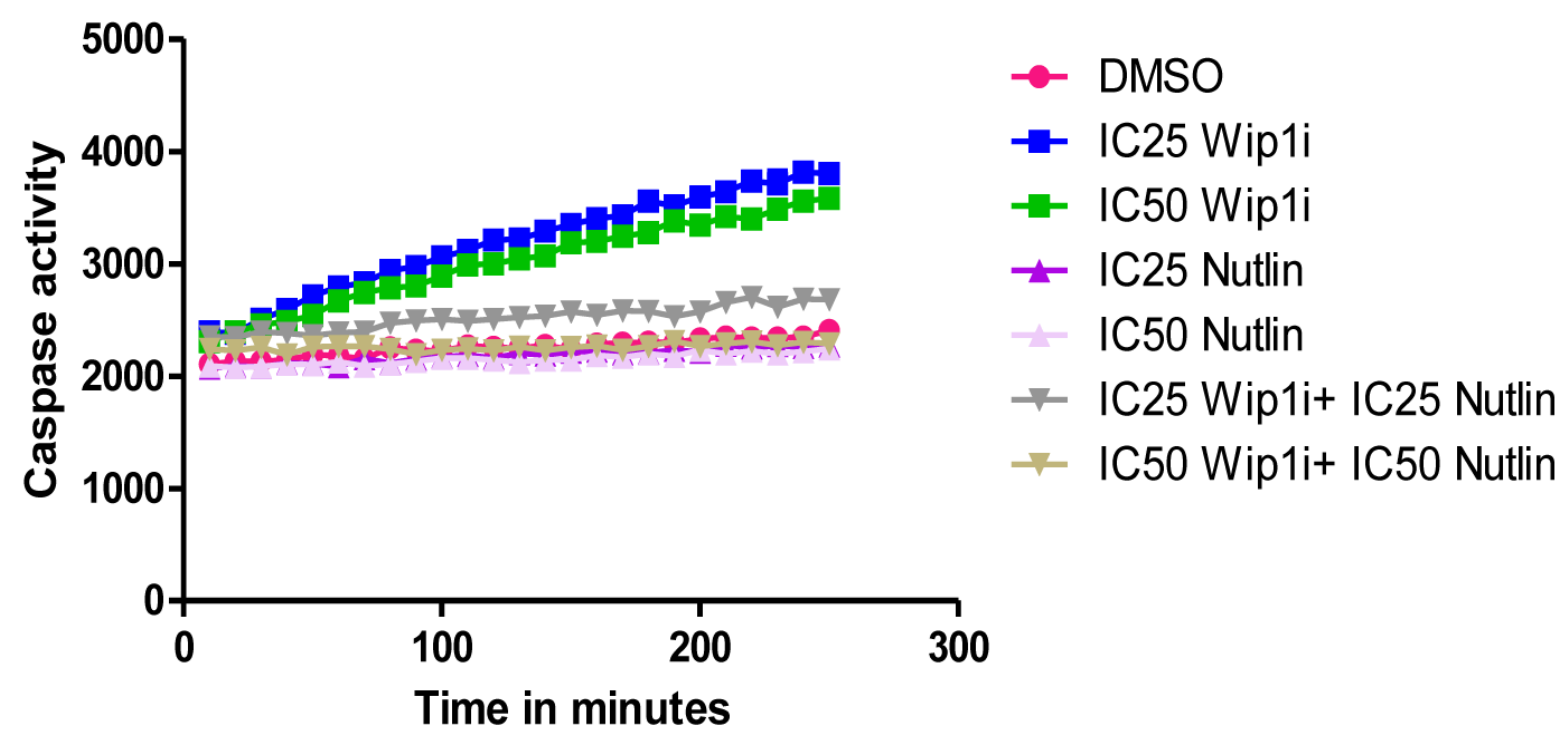

SUPPLEMENTAL FIGURE 2. Lack of caspase activation upon simultaneous inhibition of Mdm2 and Wip1. Related to Fig. 2

U2OS cells were treated as indicated, followed by harvest and lysis to assay for Caspase 3 activity after $24 \mathrm{~h}$. A fluorescent substrate to Caspase 3 was added to the lysates, and cleavage was followed over time. Note that Wip1i alone induces a moderate degree of Caspase activation, but Nutlin or the combinations do not. This assay could only be carried out in U2OS cells but not in MCF-7 cells, since the latter are lacking functional caspase 3 [66]. 


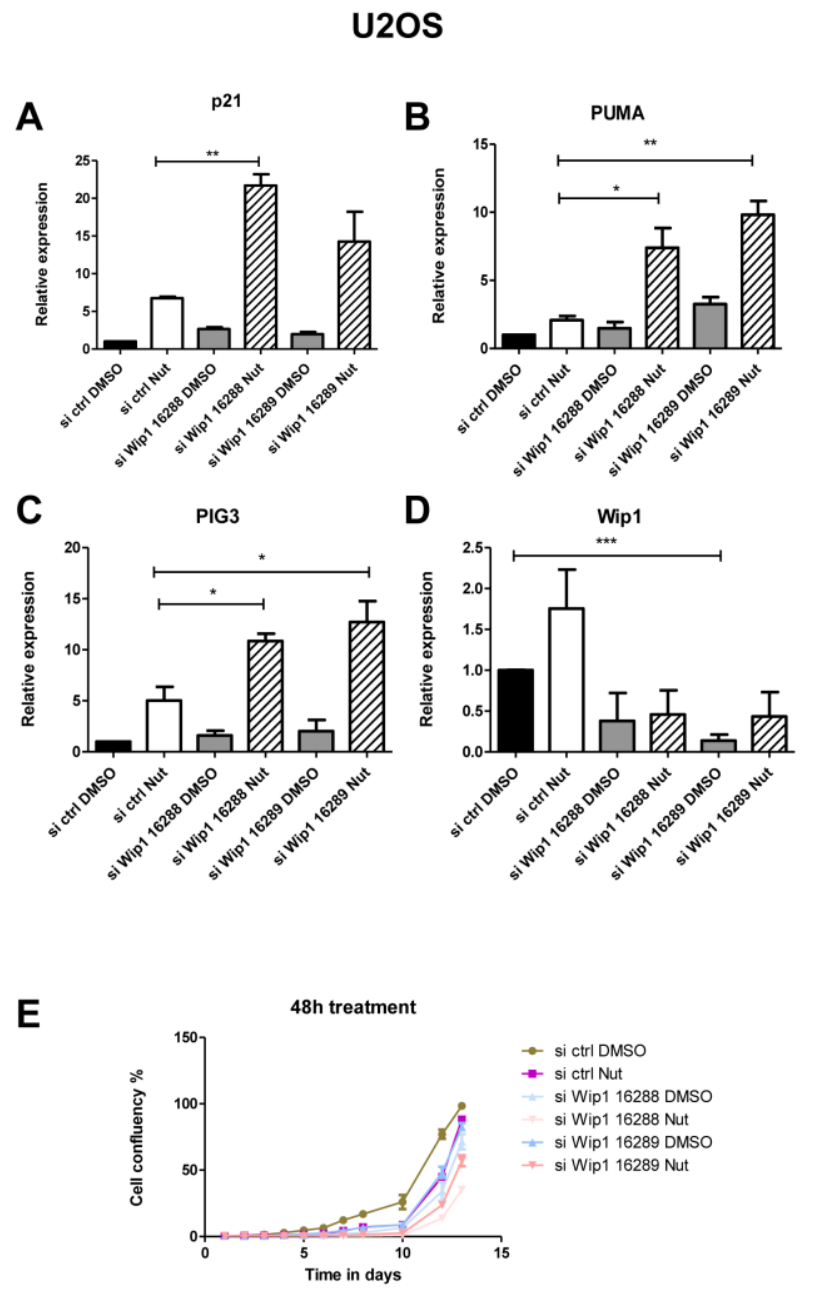

SUPPLEMENTAL FIGURE 3. Wip1 knockdown along with Nutlin treatment leads to the activation of p53 target genes and cooperatively reduces cell proliferation. Related to Fig. 3

siRNA knockdown of Wip1 was combined with Nutlin treatment in U2OS cells. Two sets of siRNA against Wip1 were used.

A-D Quantitative RT-PCR was carried out to quantify the expression of p53 target genes, namely p21, PUMA and PIG3.

E the proliferation of U2OS cells was monitored after Wip1 knockdown and/or Nutlin treatment for $48 \mathrm{~h}$. 
A

U2OS 24h treatment

$0 \mathrm{hr}$
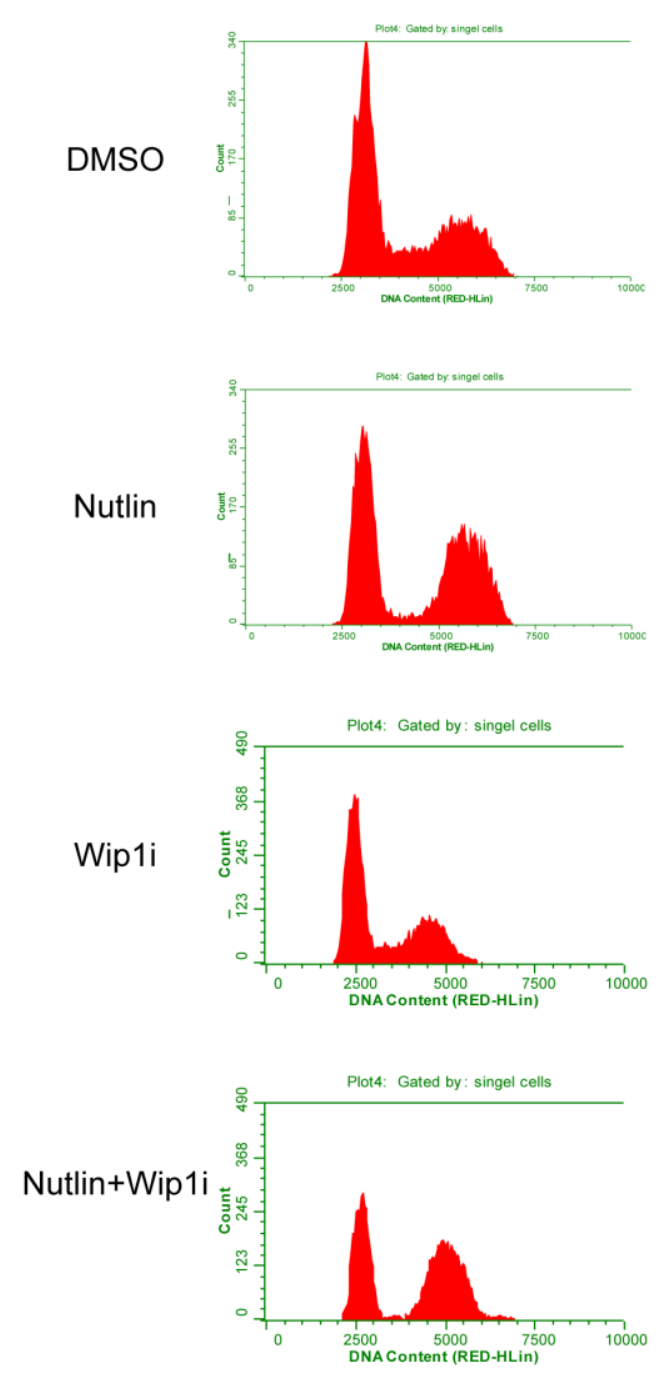

$48 \mathrm{hr}$
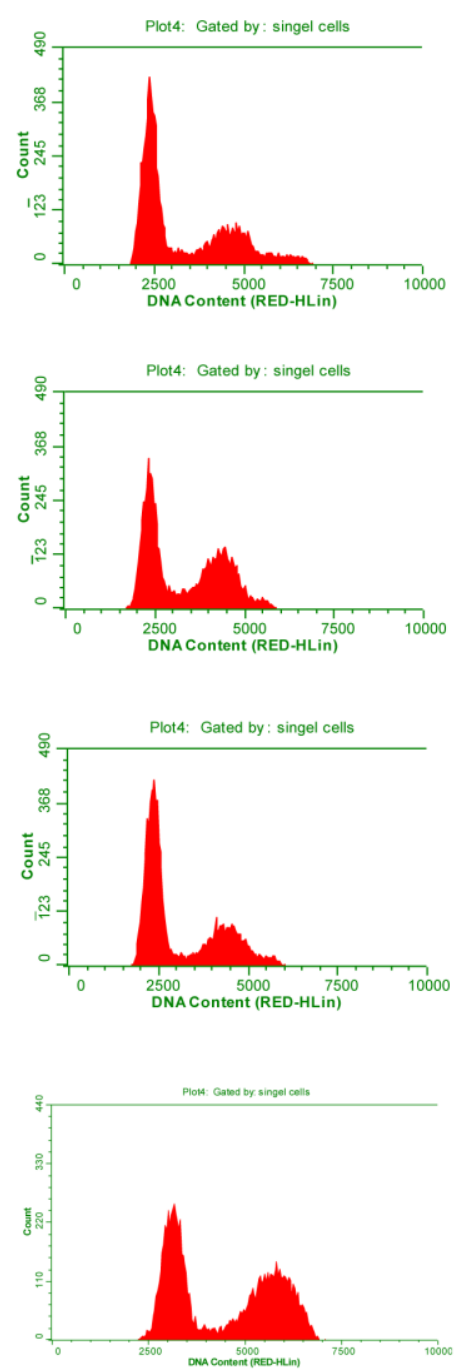

$96 \mathrm{hr}$
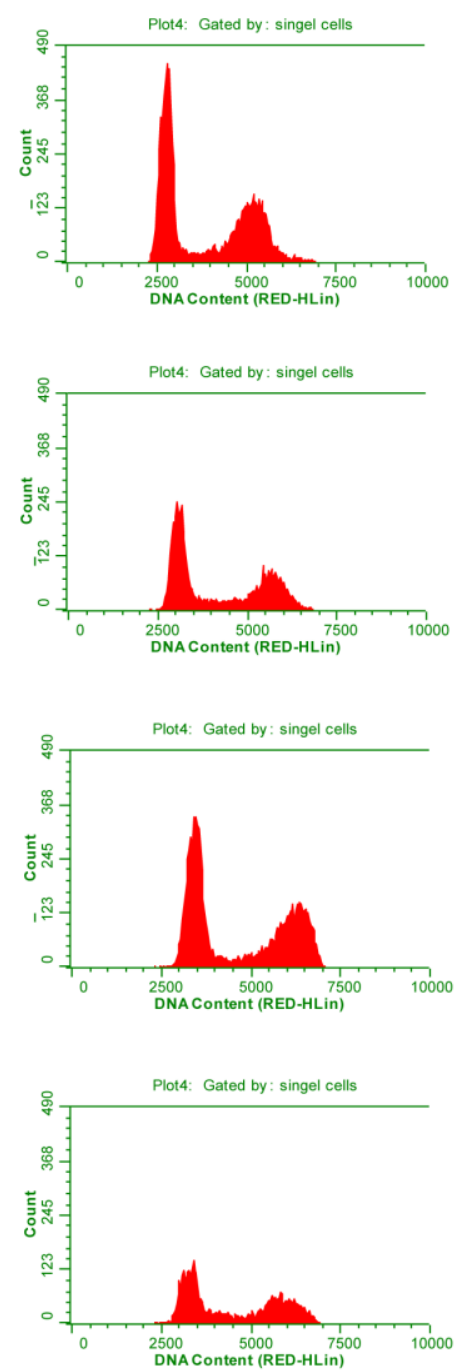
B

HCT116 p53+/+ 24h treatment

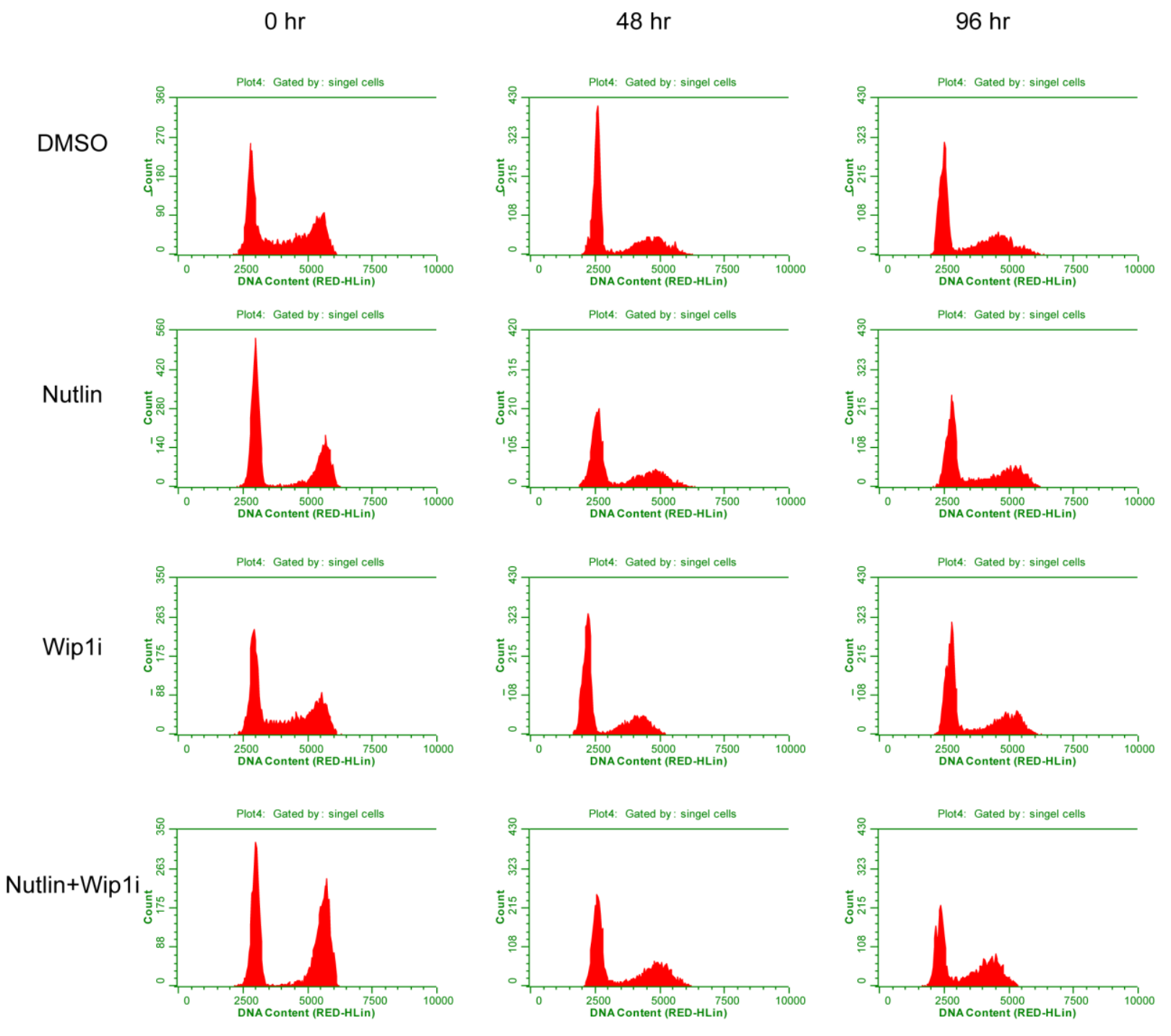


C

HCT116 p53-/- 24h treatment

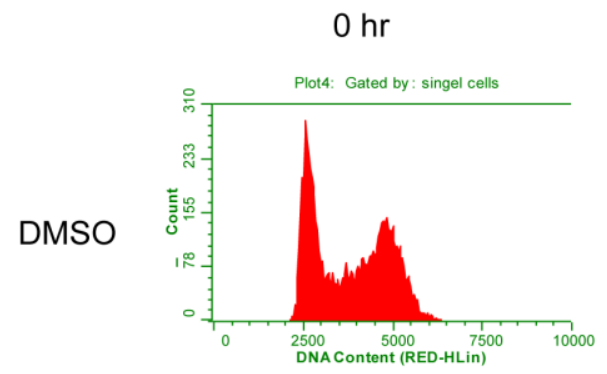

$48 \mathrm{hr}$

$96 \mathrm{hr}$
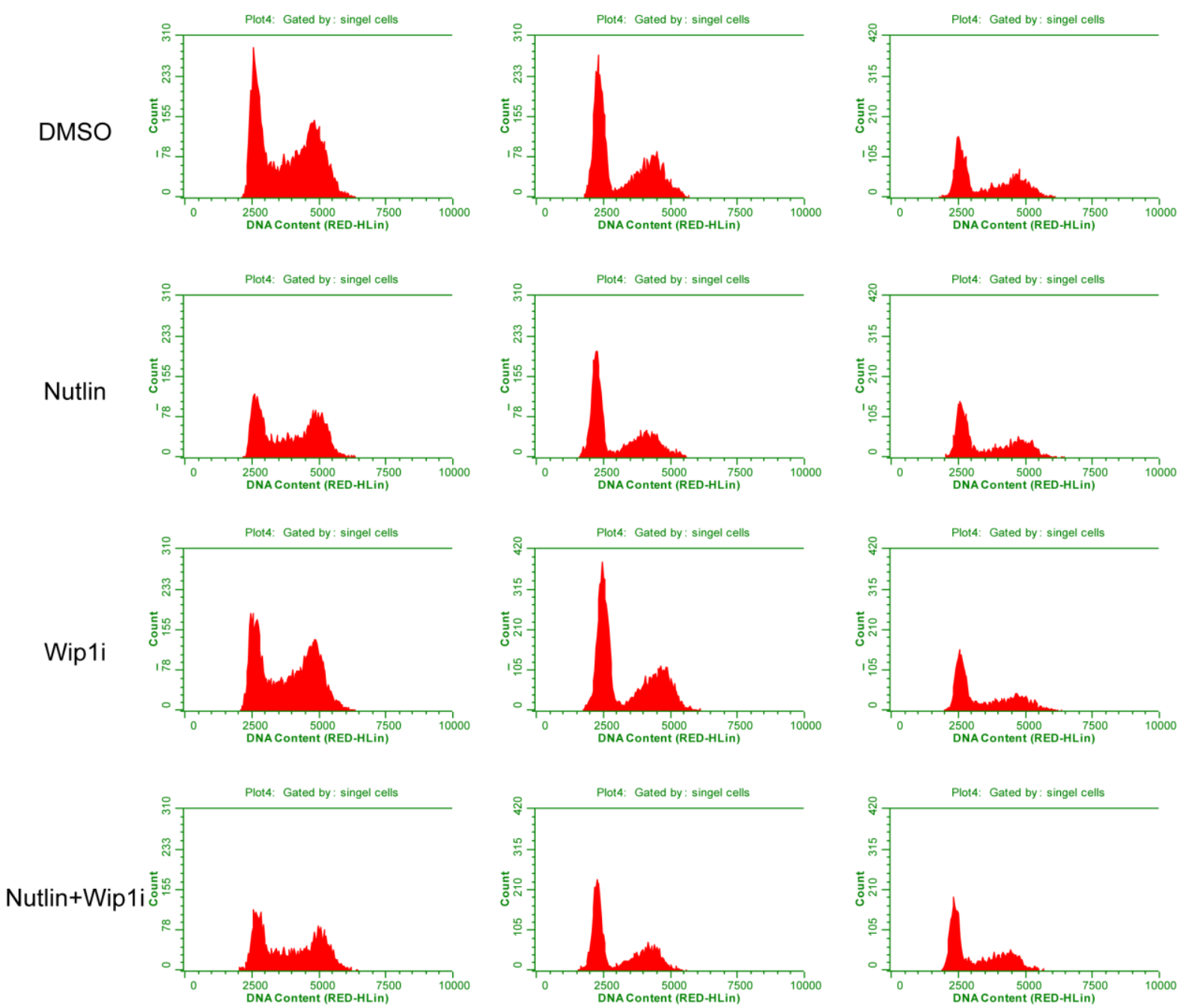
D

U2OS
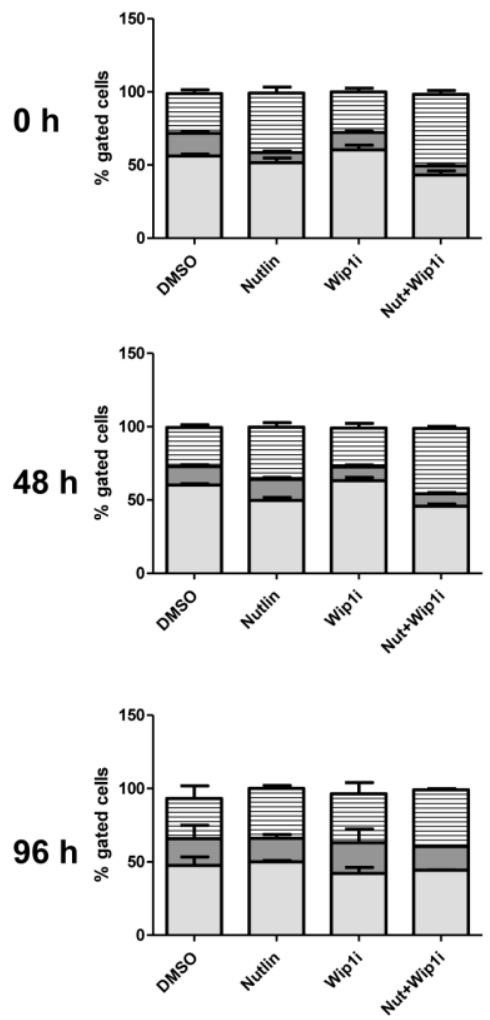

HCT116 p53+/+
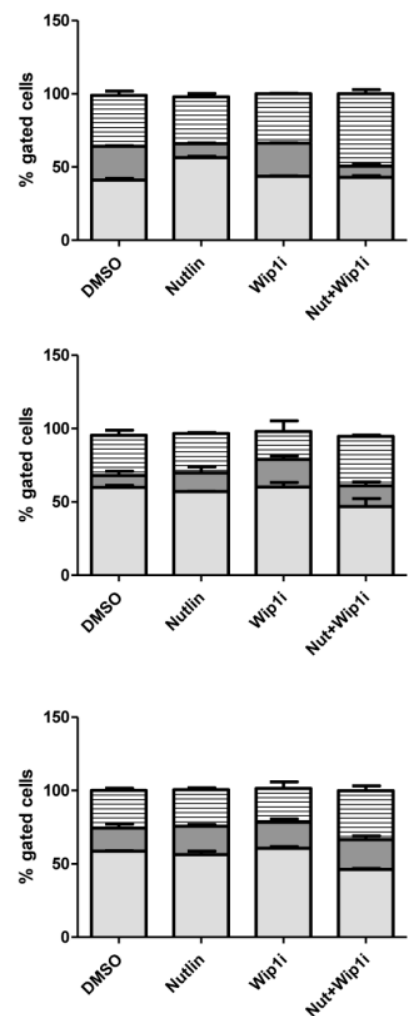

HCT116 p53-/-
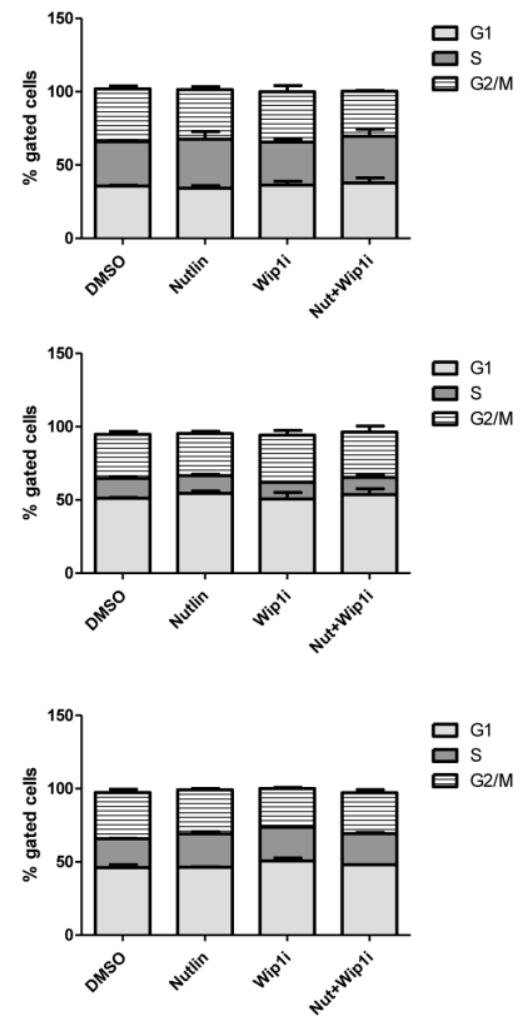

SUPPLEMENTAL FIGURE 4. Cell cycle analysis upon treatment with Nutlin, Wip1i and their combination, at different time points after treatment. Related to Fig. 6

U2OS and HCT116 (p53 proficient or deficient) cells were treated with Nutlin, Wip1i and their combination for $24 \mathrm{~h}$. The cells were then harvested for cell cycle analysis immediately (referred to as $0 \mathrm{~h}$ ), or incubated with fresh medium and harvested after $48 \mathrm{~h}$ or $96 \mathrm{~h}$. The cell cycle profiles were determined by propidium iodide staining and flow cytometry, and are provided along with the percentage of cells in each phase. 
Table S1_MCF-7_RNASeq, related to Fig. 4

MCF-7 cells were treated with $10 \mu \mathrm{M}$ of Nutlin, $10 \mu \mathrm{M}$ of GSK 2830371 or a combination of both drugs for $16 \mathrm{~h}$. Global gene expression was analyzed via next generation RNA-sequencing (cf. Fig. 4A). Normalized RNA-seq reads were analyzed by DESeq2. Base mean values, log2 fold change (FC) and adjusted $p$ values are shown for each gene.

\section{Table S2_MCF-7_C2GSEA, related to Fig. 4}

Gene set enrichment analysis (GSEA) from C2 curated gene sets (provided by the Molecular Signatures Database (MSigDB) v5.0) was performed using variance stabilized MCF-7 RNA-Seq data from Nutlin and Nutlin+Wip1i treated samples [60, 61]. The threshold of significant enrichment ( $(\mathbf{q} 0.25)$ was implied according to the GSEA standards (http://www.broadinstitute.org/gsea/doc/GSEAUserGuideFrame.html).

\section{Table S3_MCF-7_p53TSS_intensity, related to Fig. 5}

p53 enrichment was analyzed on the transcriptional start sites (TSSs) of genes that were regulated in MCF-7 cells after Nutlin and after Nutlin+Wip1i treatment. Tabs 1-4 denote the following regulation patterns: 1) genes upregulated after Nutlin and Nutlin+Wip1i treatment; 2) genes only upregulated after Nutlin+Wip1i treatment; 3) genes downregulated after Nutlin and Nutlin+Wip1i treatment; 4) genes downregulated only upon Nutlin+Wip1i treatment. Raw data for p53 ChIP-Sequencing [39] were downloaded from the Gene Omnibus database (ID GSE47043). 


\subsection{Publication III}

\section{CDK4 inhibition diminishes p53 activation by MDM2-antagonists}

Anusha Sriraman ${ }^{1}$, Antje Dickmanns ${ }^{1}$, Zeynab Najafova ${ }^{2}$, Steven A. Johnsen ${ }^{2}$, and Matthias Dobbelstein $^{1,3}$

This manuscript has been peer-reviewed and is currently under consideration at Cell Death and Disease.

\section{Own contribution:}

Experiments and data analyses for all figures except Fig5. Involved in the conception, figure arrangement and contributions in writing and revising the manuscript. 


\section{ABSTRACT}

The genes encoding MDM2 and CDK4 are frequently co-amplified in sarcomas, and inhibitors to both targets are approved or clinically tested for therapy. However, we show that inhibitors of MDM2 and CDK4 antagonize each other in their cytotoxicity towards sarcoma cells. CDK4 inhibition attenuates the induction of p53-responsive genes upon MDM2 inhibition, and p53 was also attenuated when co-depleting MDM2 and CDK4 with siRNA, compared to MDM2 single knockdown. The complexes of p53 and MDM2, as well as CDK4 and Cyclin D1, physically associated with each other, suggesting direct regulation of p53 by CDK4. Interestingly, CDK4 inhibition did not reduce $\mathrm{p} 53$ binding or histone acetylation at promoters, but rather attenuated the subsequent recruitment of RNA Polymerase II. Taken together, our results suggest that caution must be used when considering combined CDK4 and MDM2 inhibition for patient treatment. Moreover, we uncover a hitherto unknown role for CDK4 and Cyclin D1 in sustaining p53 activity.

\section{HIGHLIGHTS}

- Inhibitors of MDM2 and CDK4 antagonize one another's cytotoxicity

- CDK4 inhibition attenuates p53 activity

- The CDK4/CyclinD1 complex physically associates with p53

- CDK4 inhibition diminishes RNA Polymerase II recruitment to p53-responsive promoters

\section{SIGNIFICANCE}

CDK4 and MDM2 are subject to co-amplification in a number of malignancies, supporting the idea of combining inhibitors to both for cancer therapy. Indeed, such combinations are currently undergoing clinical testing. However, our results raise strong reservations against such an approach, since the drugs work in an antagonistic fashion. CDK4 inhibitors interfere with p53 activity and block the recruitment of RNA Polymerase II to p53-inducible promoters. Our results establish a previously unknown function of CDK4 in supporting the activity of the most frequently mutated tumor suppressor p53. 


\section{INTRODUCTION}

Cyclin-dependent kinase 4 (CDK4) is a key promoter of cell proliferation. It enables the transition through the $\mathrm{G} 1$ phase of the cell cycle, a prerequisite for subsequent entry to $\mathrm{S}$ phase and cell division. Tumor cells often activate CDK4 to ensure proliferation, either by silencing genes that encode CDK4 antagonists or by enhancing CDK4 expression, e. g. through gene amplification. Pharmacological inhibitors of CDK4 have proven to be effective in cancer treatment, leading to FDA approval of Palbociclib (PD0332991), Ribociclib (LEE011) and Abemaciclib (LY2835319) [181].

The MDM2 oncoprotein has also been extensively evaluated as a drug target. MDM2 antagonizes the tumor suppressor p53 by physical interaction and subsequent ubiquitination of p53. The interaction of p53 and MDM2 is amenable to targeting by small compounds, with Nutlin-3a (referred to here as "Nutlin") representing the prototype [166] and many similar and further refined compounds being developed ever since [182, 183]. Pre-clinical analysis of MDM2 inhibitors have raised high expectations, especially when treating sarcoma [184] or glioblastoma [185] which contain MDM2 gene amplifications. Clinical studies using MDM2-targeting drugs [186], however, have currently not met the initial expectations [165], at least not when used as single drugs. This has spurred the search for optimized combinations of MDM2 inhibitors with other cancer drugs.

Certain sarcomas, specifically liposarcomas, represent particularly promising cancer entities for treatment with MDM2 antagonists. These tumors contain amplifications of the MDM2 gene in more than $90 \%$ of all cases [187], and liposarcoma-derived cell lines undergo apoptosis when treated with MDM2antagonizing drugs $[184,188]$. As expected, the response requires a wild-type p53 status and MDM2 overexpression [189]. However, attempts to treat liposarcoma patients with MDM2 antagonists have so far not yielded the expected clinical success $[167,190]$.

The amplification of the MDM2 gene in sarcomas is often associated with CDK4 amplifications [187]. Other examples of tumors containing both amplifications include melanomas [191] and periosteal osteosarcomas [192]. Both genes are located close to each other on chromosome 12q13-15, but nonetheless, the amplifications appear independent in most cases [187, 193]. The co-amplification of both genes might constitute tumor cell addiction to the simultaneous activity of both gene products. This argued that targeting both MDM2 and CDK4 should yield synergistic tumor cell killing. And indeed, a recent report argued that this synergism might be achievable [194]. Moreover, a combination of MDM2 and CDK4 inhibitors is currently being evaluated in a Phase I clinical study (NCT02343172). However, in the previous report [194], the impact of drug combinations on tumor growth was only marginally increased when compared to CDK4 inhibitor alone. Thus, potential synergies between CDK4 and MDM2 inhibitors remain to be investigated.

Here we show that the inhibition of CDK4 attenuates MDM2 inhibitor-induced activity of p53, leading to decreased rather than synergistic cytotoxicity. In parallel, the complexes of MDM2 and p53, as well as CDK4 and Cyclin D1, physically associate with each other. CDK4 inhibition still allows efficient binding 
of p53 to its target genes. In contrast, combined inhibition of CDK4/6 and MDM2 led to diminished RNA Polymerase II recruitment and thus decreased transcription of p53 target genes.

\section{RESULTS}

\section{Inhibitors of CDK4 and MDM2 lack synergistic cytotoxicity towards sarcoma cells}

Given the co-amplification of the MDM2 and CDK4 genes in sarcoma, we sought to test whether the combined inhibition of both gene products might synergistically eliminate cancer cells. We treated SJSA cells (osteosarcoma cells with amplifications of MDM2 and CKD4, cf. www.cbioportal.org and Figure 3.3.S1 A) with combinations of the CDK4 inhibitor Palbociclib (PD0332991) and the MDM2 antagonist Nutlin. As expected, Nutlin induced p53 accumulation and its target genes p21 and MDM2 (Figure 3.3.S1B), and PD0332991 abolished the phosphorylation of the CDK4 substrate pRb at Serine 807/811 [195] (Figure 3.3.S1 C). Nutlin also reduced pRb phosphorylation, likely due to the induction of the CDK4 inhibitor p21. Importantly, Nutlin profoundly decreased the viability of SJSA cells, as reported previously [196], and Palbociclib also reduced their viability (Figure 3.3.1A,B).

A

SJSA

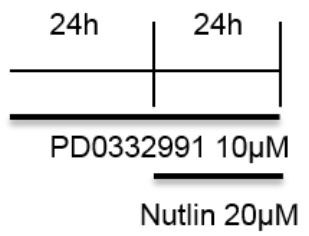

B

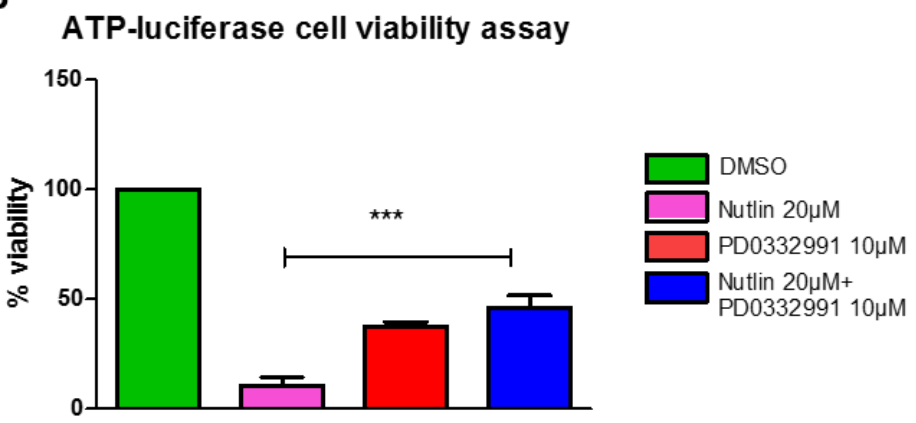

Figure 3.3 1 CDK4 inhibitors and MDM2 antagonists fail to synergize with regard to cytotoxicity towards sarcoma cells

A. SJSA cells were treated with DMSO, Nutlin, PD0332991 and its combination at the indicated concentrations adhering to the depicted schedule.

B. Cell viability was measured by quantification of the ATP content. The combination of PD0332991 and Nutlin showed a protective effect in comparison to Nutlin alone.

Strikingly, however, Palbociclib completely failed to enhance the cytotoxic effects elicited by Nutlin. Instead, Nutlin-treated cells even survived to a significantly greater extent when co-treated with Palbociclib. Similarly, the long-term survival of SJSA cells was strongly decreased by Nutlin alone, but was rescued by co-treatment with Palbociclib (Figure 3.3.1C,D). 
C

SJSA

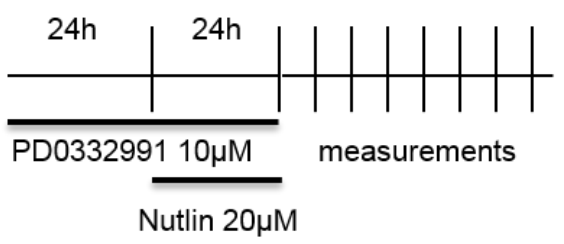

D

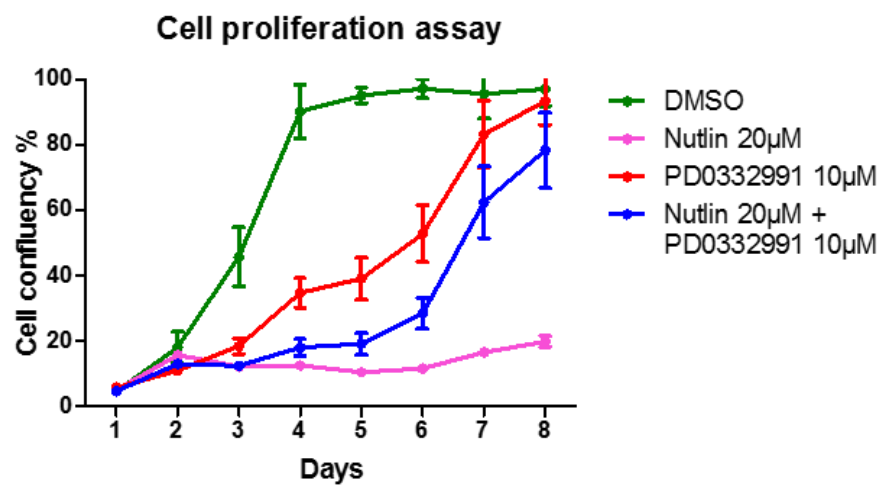

Figure 3.3 1 CDK4 inhibitors and MDM2 antagonists fail to synergize with regard to cytotoxicity towards sarcoma cells.

C. Schedule to determine cell proliferation upon drug treatment.

D. Cell proliferation was assessed by daily measuring the confluency of cells using a Celigo cell cytometer. The medium was changed every 24 hours. Nutlin treatment reduced cellular proliferation. However, pretreatment with PD0332991 in combination with Nutlin led to increased cell numbers, indicating that the two drugs do not synergize but rather act in an antagonistic fashion. Mean of three biological replicates.

Moreover, SJSA cells treated with Nutlin alone displayed morphology with round and shrunk cells, corresponding to apoptosis [197] (Figure 3.3.1E).

SJSA

DMSO

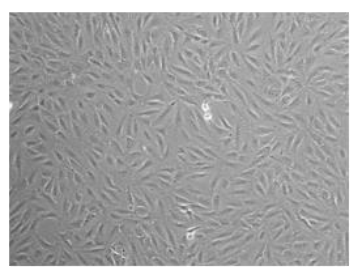

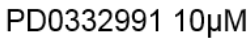

$24 \mathrm{~h}$

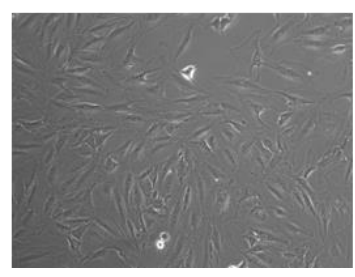

Nutlin $20 \mu \mathrm{M}$

$24 \mathrm{~h}$

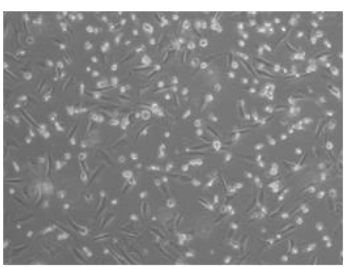

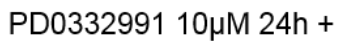

Nutlin $20 \mu \mathrm{M} 24 \mathrm{~h}$

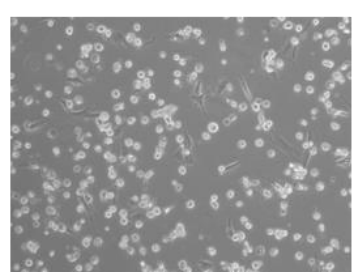

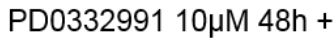

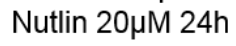

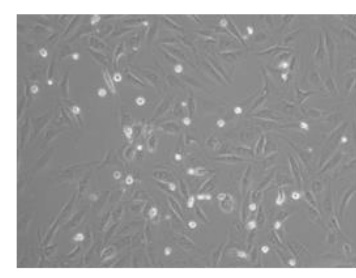


Figure 3.3 1 CDK4 inhibitors and MDM2 antagonists fail to synergize with regard to cytotoxicity towards sarcoma cells.

E. Morphology of SJSA cells, observed by bright field microscopy. Upon treatment with Nutlin, the cells shrank and detached. Pretreatment with PD0332991 protected the cells from this cytotoxic effect of Nutlin. However, removal of PD0332991 followed by Nutlin treatment did not result in cell protection.

Again, this occurred to a lesser extent when the cells had first been treated with Palbociclib. Combination of the drugs at different concentrations in various MDM2-amplified sarcoma cell lines also revealed no synergism with regard to viability of cells (Figure 3.3.S1 D-G; note that here, viability was assayed immediately after a 72 hrs treatment, without allowing the cells to further proliferate). Taken together, these results strongly suggest that at least under some circumstances Palbociclib is capable of antagonizing the cytotoxic activity of Nutlin.

\section{CDK4 is required for p53-induced gene expression}

To further investigate why CDK4 inhibition attenuates Nutlin-induced cell death, we asked whether CDK4 inhibitors might interfere with p53-induced gene expression. A panel of sarcoma cells, including SJSA cells, as well as CRL3043 [198], CRL3044 [198] and GOT-3 [199] (the latter three derived from welldifferentiated liposarcoma and with amplifications of CDK4 and MDM2 genes), was treated with Palbociclib to inhibit CDK4, as well as Nutlin to block the MDM2-p53-interaction. The levels of p53responsive gene products were then assessed by immunoblot analysis. Nutlin alone increased the levels of p53 and its target gene products MDM2 and CDKN1A/p21, as expected. In contrast, Palbociclib alone had little effect on them, while it did reduce the amount of phosphorylated pRb. Importantly, however, in combination with Nutlin, Palbociclib markedly decreased the protein levels of the p53 target gene products MDM2 and p21 (Fig. 3.3.2A).

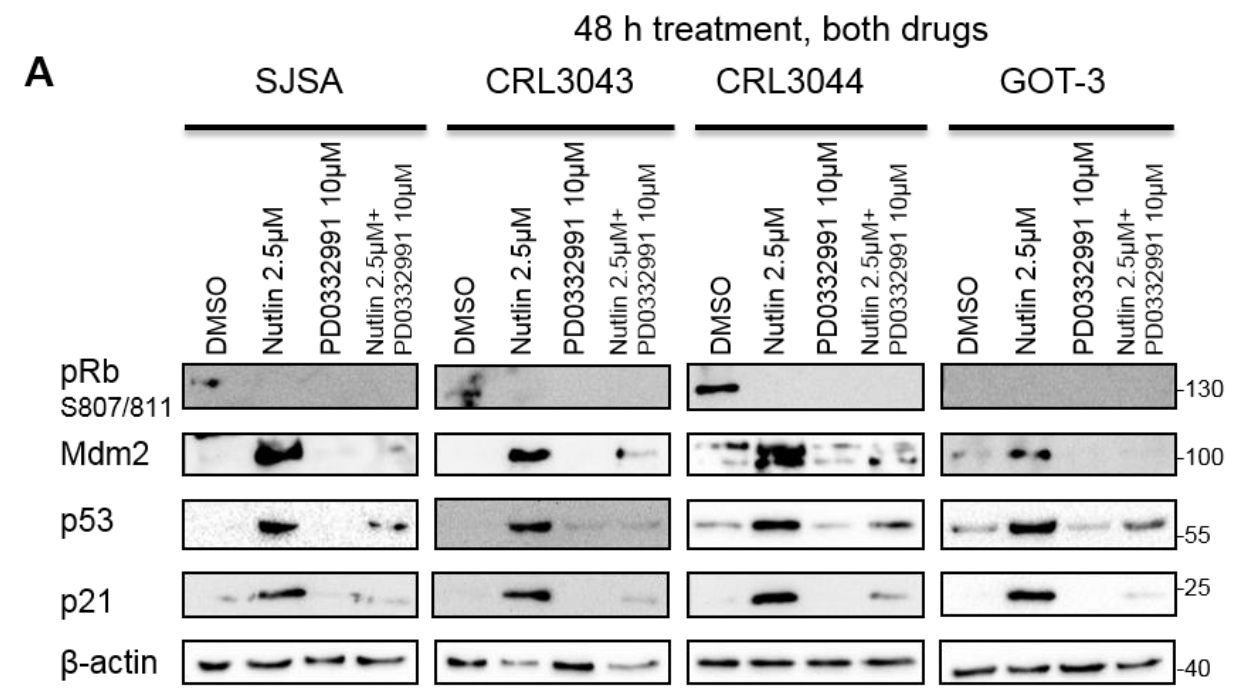

Figure 3.32 CDK4 is required for p53-induced gene expression. 
A. SJSA (osteosarcoma), CRL3043, CRL3044 and GOT-3 (all liposarcoma) cells were treated with DMSO, Nutlin, PD0332991 or the drug combination for 48 hours. Cells were harvested for immunoblot analysis to detect p53 and its target gene products p21 and MDM2. Upon Nutlin treatment, increased levels of MDM2, p53 and p21 were observed. However, the combination of Nutlin with PD0332991 decreased p53-induced target gene expression. pRb phosphorylated at $807 / 811$ was detected as a positive control for the activities of Nutlin and PD0332991. B-actin served as loading control.

To determine whether the impairment of p53 target gene expression was specific to Palbociclib or whether it was due to inhibition of CDK4/6 kinase activity, in general, we treated SJSA cells with alternate, FDA approved CDK4/6 inhibitors, namely LEE011 (Ribociclib) and LY2835219 (Abemaciclib), alone or in combination with Nutlin. Again, p53 target gene expression was decreased when Nutlin was combined with the CDK4/6 inhibitors, at the protein (Figure 3.3.2B) and mRNA (Figure 3.3.2C) levels. Thus, CDK4 inhibition generally reduces p53 activity. The failure of Nutlin to fully induce pre-apoptotic genes in the presence of CDK4 inhibitor may also explain why CDK4 inhibition attenuates the cytotoxic effects of Nutlin (Figure 3.3.1).
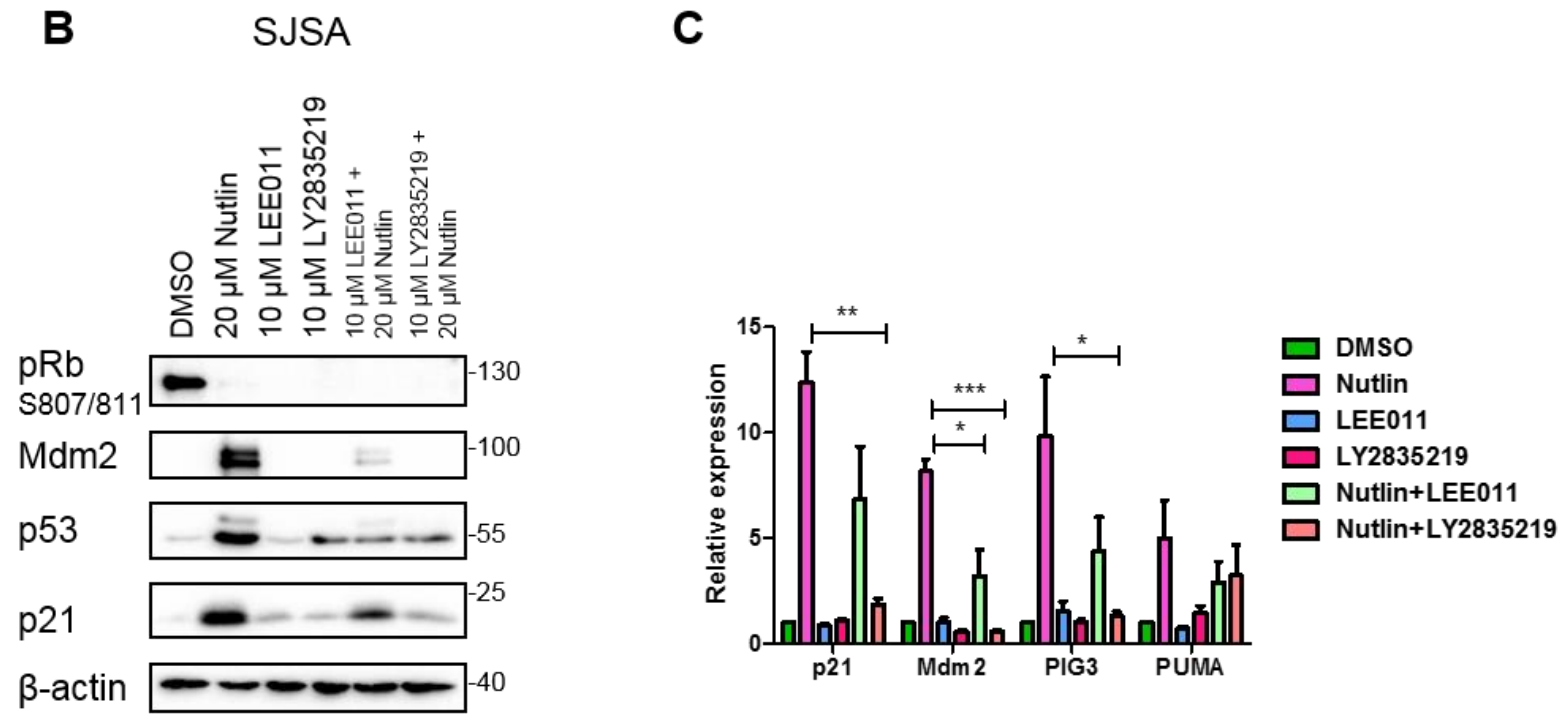

Figure 3.32 CDK4 is required for p53-induced gene expression.

A. SJSA cells were treated with the alternate CDK4/6 inhibitors Ribociclib (LEE011) and Abemaciclib

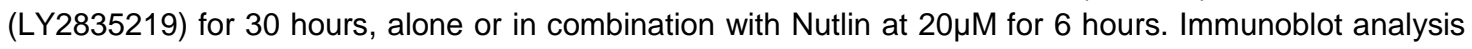
was performed as in A. Like Palbociclib (PD0332991), the combination of Nutlin with alternate CDK4/6 inhibitors diminished p53 target gene expression when compared to Nutlin treatment alone.

B. SJSA cells were treated with the alternate CDK4/6 inhibitors Ribociclib (LEE011) and Abemaciclib (LY2835219), alone and in combination with Nutlin as in B. mRNA levels corresponding to p53 target gene expression were assessed by quantitative Real Time-PCR. RPLPO was used as a reference gene. Again, p53-induced gene expression was found diminished by CDK4/6 inhibitors. Mean of three biological replicates. 
We next increased p53 activity by inducing a DNA damage response, which enhances p53 phosphorylation through the kinases ATM and Chk2 [200]. To this end we used neocarzinostatin (NCS), a radiomimetic compound that induces double strand DNA breaks in a manner similar to ionizing radiation [201]. Strikingly, Palbociclib pretreatment strongly decreased the accumulation of p53 and its target gene product p21 in response to NCS (Figure 3.3.2D).

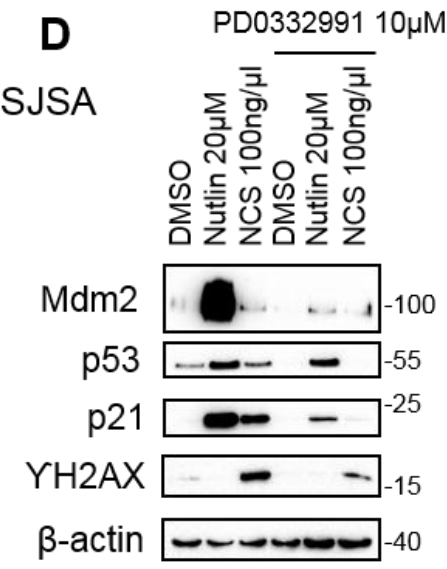

Figure 3.32 CDK4 is required for p53-induced gene expression.

C. SJSA cells were treated with DMSO, Nutlin, Neocarzinostatin (NCS), PD0332991 and their combinations at the indicated concentrations for 6 hours. Lysates were subjected to immunoblot analysis. Upon NCS treatment, increased levels of p21 and YH2AX were found, indicative of a DNA damage response. As in the case of Nutlin, NCS-induced p53 activity, revealed by p21 accumulation, was found reduced by the CDK4/6 inhibitor.

Hence, CDK4 inhibition can also interfere with the p53-inducing ability of DNA damaging drugs, giving rise to caution when combining CDK4 inhibitors with conventional chemotherapy in cancer treatment. Furthermore, we performed analogous investigations replacing pharmacological inhibitors with siRNAs targeting MDM2 and CDK4. This revealed corresponding changes in p53-induced p21, i.e. induction by MDM2 knockdown alone, but far less induction by the simultaneous depletion of MDM2 and CDK4 (Figure 3.3.2E). 

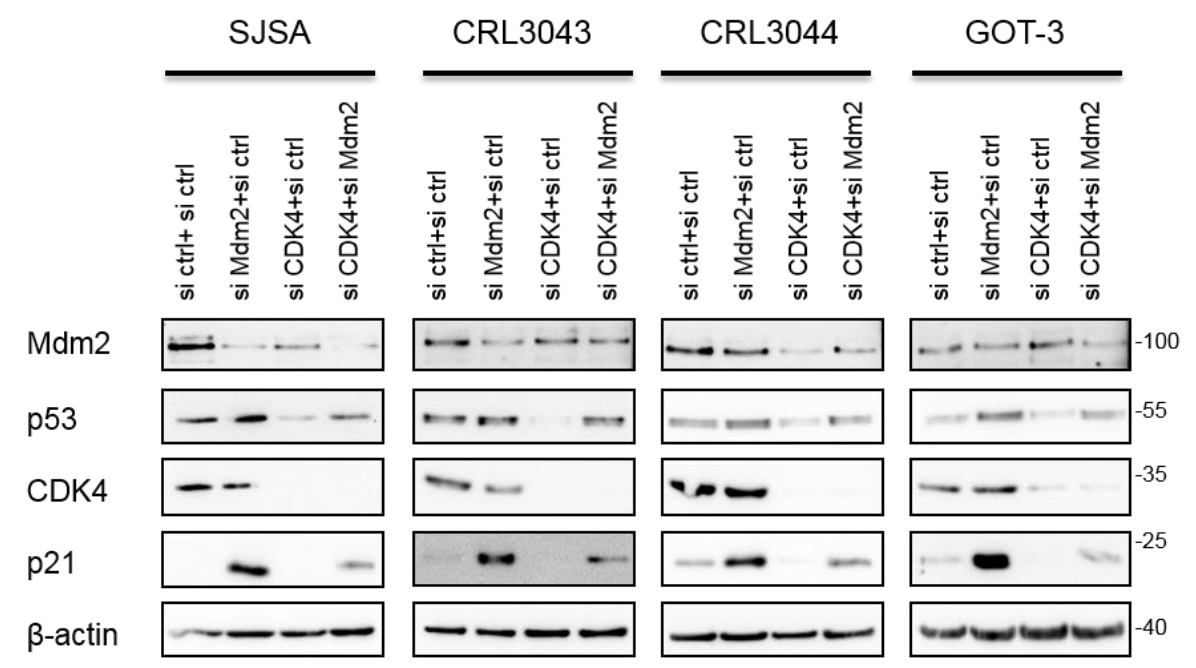

GOT-3

Figure 3.32 CDK4 is required for p53-induced gene expression.

D. SJSA, CRL3043, CRL3044 and GOT-3 cells were depleted of endogenous MDM2, CDK4 by siRNA transfection, in comparison to control (ctrl) siRNA. Cells were harvested for immunoblot after 48 hours to detect p53 and its target gene product p21. Upon depletion of MDM2, the expected increase in p21 and p53 levels was observed. In contrast, the co-depletion of CDK4 along with MDM2 induced p21 and p53 levels only to a lesser extent.

Similarly, impaired p53 target gene expression upon CDK4 inhibition was also observed when using different time schedules, drug concentrations, or the alternate MDM2 antagonist RG7388 (Figure 3.3.S2, A-E). Moreover, to exclude any role of the p53 kinase HIPK2 $[100,101]$ in this context, we performed a parallel experiment replacing CDK4 inhibitors with the HIPK2 inhibitor A64 (PubChem Substance ID 329826044) but did not observe any detectable change in p53 activity (Figure 3.3.S2F,G). Taken together, these results strongly suggest that CDK4 inhibition or depletion severely diminishes the transcriptional activity of $p 53$ in response to MDM2 antagonists. This raises an important potential caveat regarding the combination of CDK4 inhibitors with p53-activating drugs for cancer therapy.

\section{Short-term reactivation of CDK4 is sufficient to rescue p53 activity}

To test whether the impact of CDK4 inhibition on p53 activity is mediated by cell cycle arrest, we analyzed the timing needed for the two drugs to interact. One possibility for the observed effects is that a cell cycle arrest induced by CDK4 inhibition might impair p53 activity. As expected, flow cytometric analysis verified that Palbociclib arrested the majority of treated cells in G1 (Figure 3.3.3A,B). 


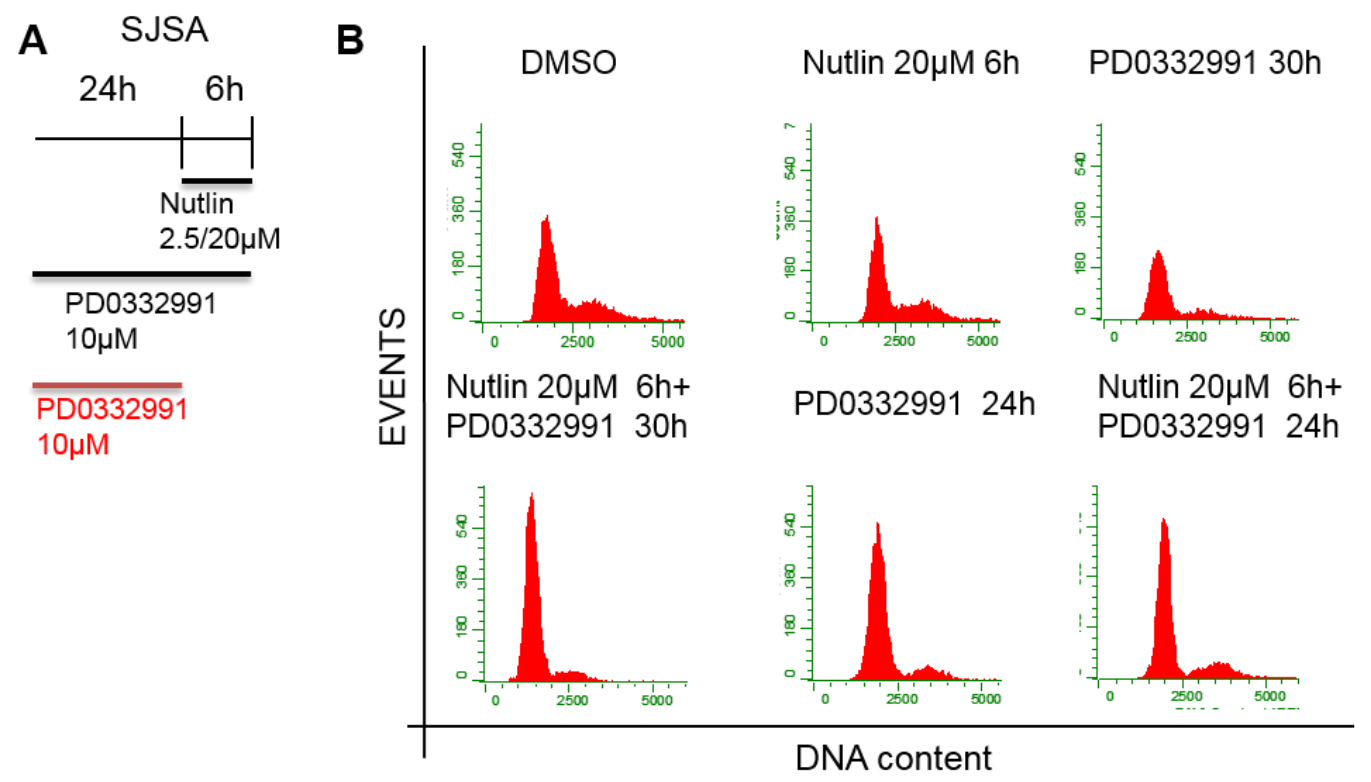

Figure 3.33 Short-term reactivation of CDK4 is sufficient to rescue p53 activity which is independent of cell cycle.

A. SJSA cells were treated as indicated in the schedule.

B. Flow cytometry analysis of the DNA content. PD0332991 induced cell cycle arrest in G1, irrespective of the (short-term) Nutlin treatment.

Although cell cycle arrest is not generally considered as a way to inhibit p53 activity, we still investigated whether this arrest in G1 represents the reason for the attenuated p53 response. To this end, we removed the CDK4/6 inhibitor from the cells for 6 hours (compared to continued CDK4 inhibition) and then immediately treated with Nutlin. This brief removal of the CDK4 inhibitor was not enough to resume the cell cycle, at least as far as can be judged by propidium iodide staining (Figure 3.3.3B). However, p53 activity was still markedly increased upon Palbociclib removal, when compared to cells that were treated with Nutlin in the continued presence of Palbociclib, as determined by immunoblot analysis of p53 target gene products (Figure 3.3.3C) and also by assessing the mRNA levels corresponding to such genes (Figure 3.3.3D). Thus, we conclude that CDK4 activity is required for maximal p53-induced gene expression, regardless of cell cycle progression. 


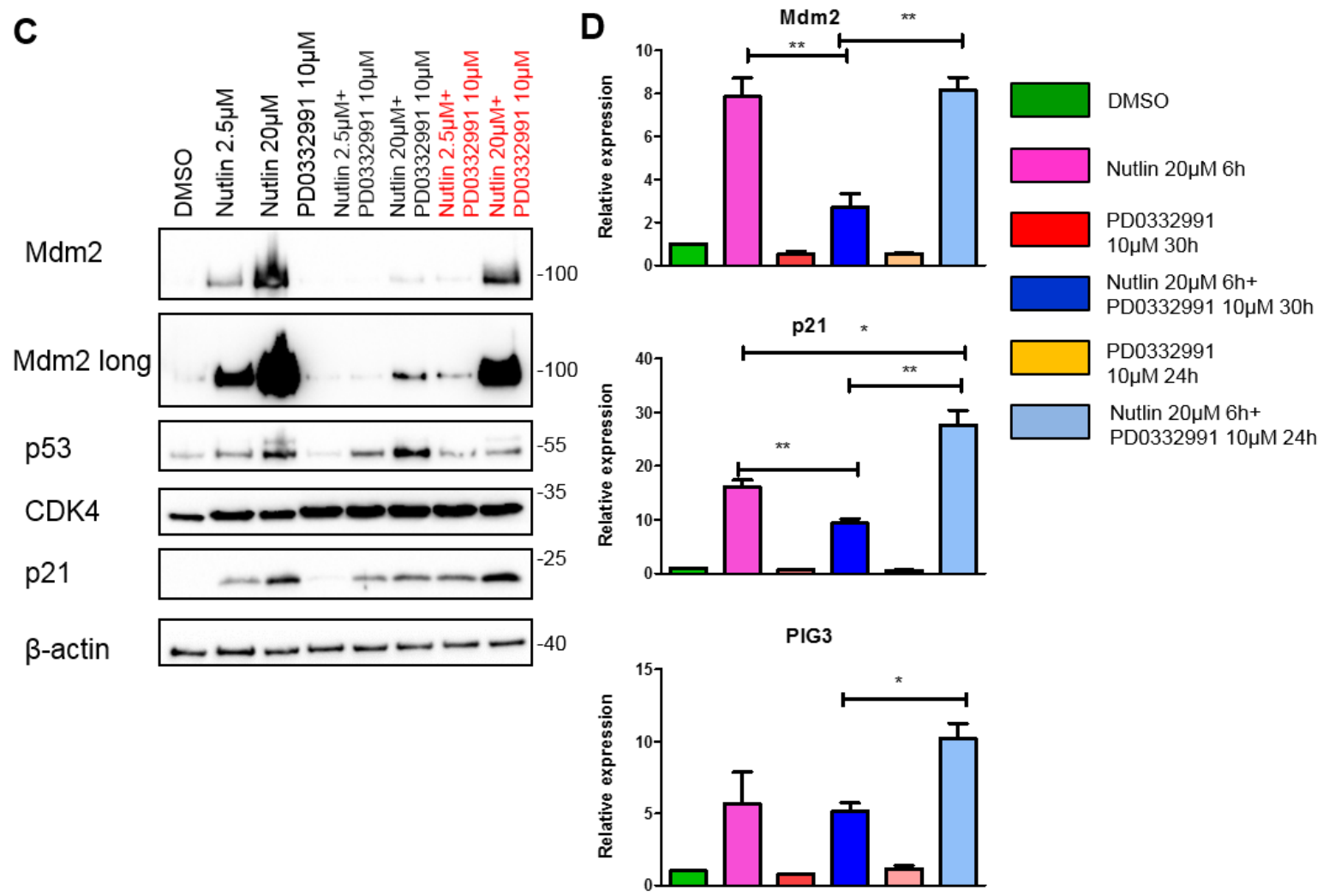

Figure 3.33 Short-term reactivation of CDK4 is sufficient to rescue p53 activity which is independent of cell cycle.

C. To investigate the p53 target gene expression, immunoblot analysis was carried out. Nutlin treatment led to the accumulation of p53, p21 and MDM2, which was diminished by PD0332991. Upon removal of PD0332991 during Nutlin treatment, however, p21 and MDM2 levels were restored.

D. Cells were treated as in A-C, followed by quantitative RT-PCR to quantify the expression of the p53 target genes MDM2, p21 and PIG3, in comparison to the reference gene RPLPO. Nutlin induced these genes while PD0332991 significantly decreased their expression levels. Removal of PD0332991 during Nutlin addition reactivated p53 target gene expression. Mean of three biological replicates.

\section{CDK4 inhibition attenuates the expression of a broad range of p53-induced genes}

Next, we assessed the extent to which the induction of genes by p53 is affected by CDK4 inhibition, and whether CDK4 inhibition might display a similarly broad impact on unrelated gene sets. We treated SJSA cells with Palbociclib and/or Nutlin (Figure 3.3.4A), followed by next generation RNA sequencing analysis (RNA-Seq). This approach revealed that most p53-responsive genes, as identified through their induction by Nutlin, were expressed to a lesser degree when cells were pretreated with the CDK4/6 inhibitor (Figure 3.3.4B). 
A SJSA

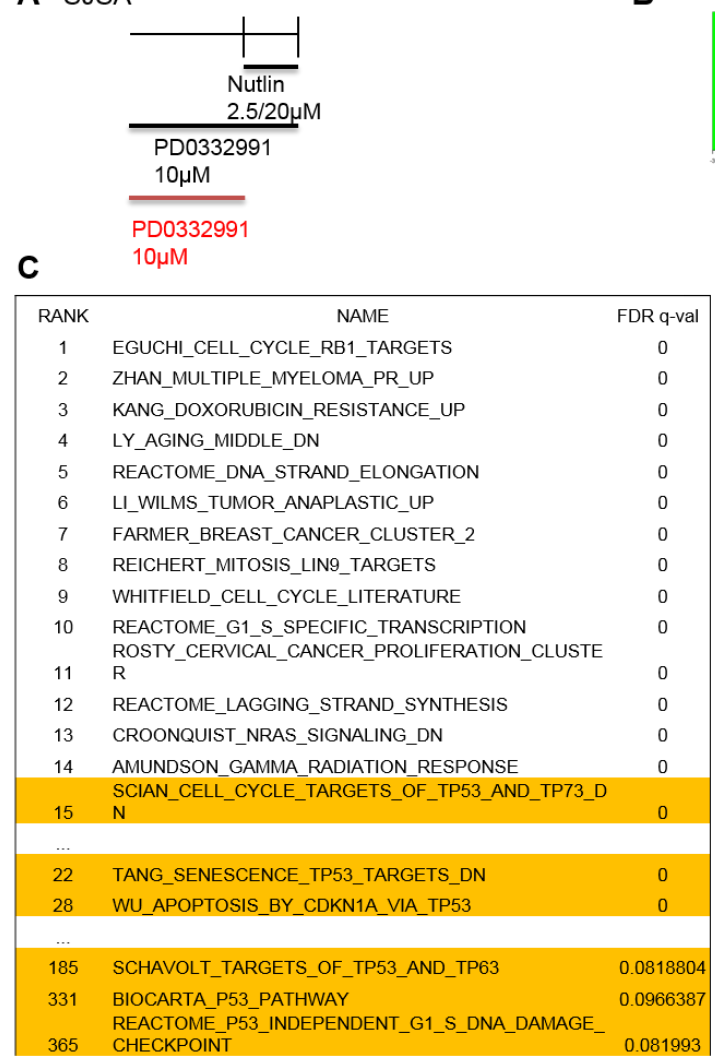

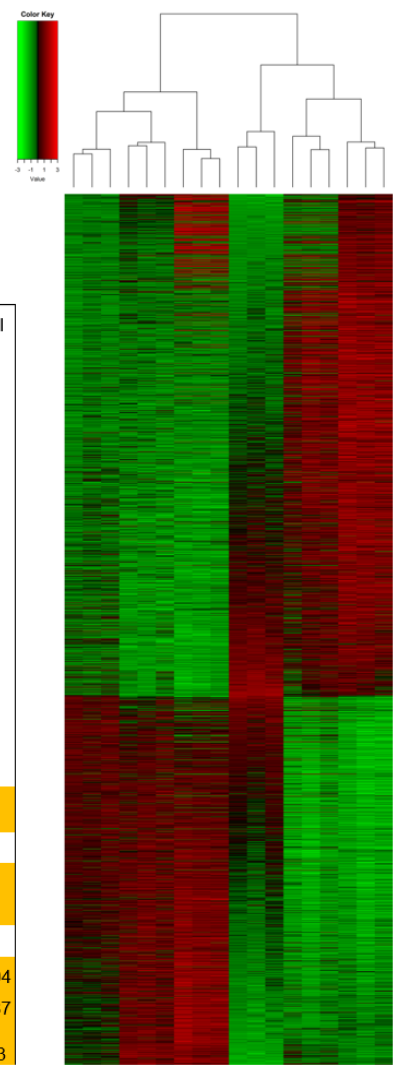

$\overline{\mathrm{PD} 30} \overline{\mathrm{PD} 24} \overline{\mathrm{DMSO}} \overline{\mathrm{N}+\mathrm{PD}} \overline{\mathrm{N}+\mathrm{PD}} \overline{\mathrm{N}}$

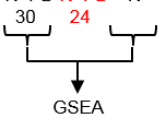

Figure 3.3 4 CDK4 inhibition attenuates the expression of a broad range of p53-responsive genes.

A. SJSA cells were treated with DMSO, Nutlin, PD0332991 and its combination as indicated in the schedule, followed by RNA deep sequencing analysis.

B. Heatmap depicting differentially regulated genes sorted according to the z-scores of Nutlin vs DMSO. Only genes with basemean $>20, \log 2$ fold $>1, \log 2$ fold $<-1$, and adjusted $p$ value $<0.05$ were taken into consideration. 6 samples with biological triplicates are represented. $\mathrm{N}$ refers to Nutlin, PD refers to PD0332991, and DMSO as control. RNA-Sequencing data of this study were submitted to the GEO, GSE113369.

C. Gene Set Enrichment Analysis (GSEA) from C2 curated gene sets, provided by the Molecular Signatures Database (MSigDB) v5.0 [202], was performed using variance stabilized RNA-Seq reads from Nutlin (represented as N) and Nutlin+PD0332991 (represented as N+PD30) treated samples. The table was generated by selecting False Discovery Rate (FDR) $<25 \%$ and Enrichment Score (ES) in the descending order.

Comparing Nutlin-treated cells with or without CDK4 inhibitor, it was still the p53-responsive genes that were differentially expressed to the greatest extent after the cell cycle targets, as determined by gene set enrichment analysis (GSEA) (Figure 3.3.4C-D). 
D
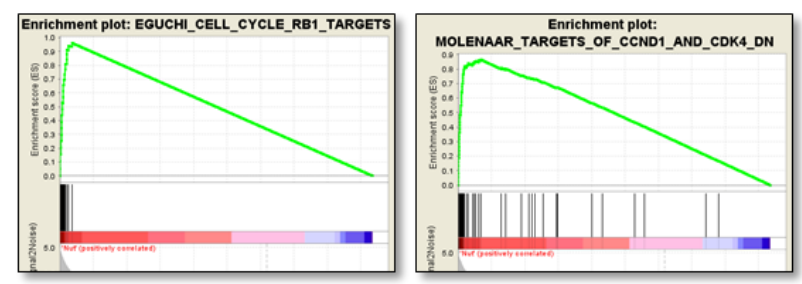

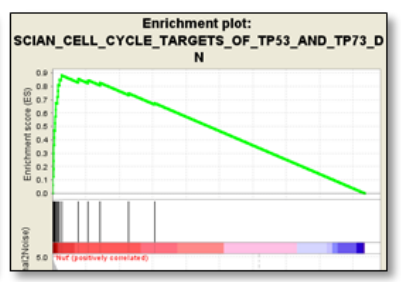

Figure 3.3 4 CDK4 inhibition attenuates the expression of a broad range of p53-responsive genes.

D. Selected enrichment plots from gene sets induced by Nutlin vs Nutlin+PD0332991 are provided as examples.

We conclude that, when cells are subjected to MDM2 inhibition, a CDK4/6 inhibitor specifically attenuates the expression of p53-responsive genes more than any other distinguishable group of genes except the cell cycle regulators. This suggests that CDK4 activity has a direct and specific impact on p53-induced transcription.

\section{p53 physically interacts with CDK4 and Cyclin D1}

To elucidate how CDK4 might affect the activity of p53, we tested whether the two molecules might physically associate with each other. We tested this in cell lysates from SJSA cells treated with a proteasome inhibitor. Indeed, co-immunoprecipitation analysis revealed the interaction of the MDM2-p53 and the CDK4-Cyclin D complexes (Figure 3.3.5A). 


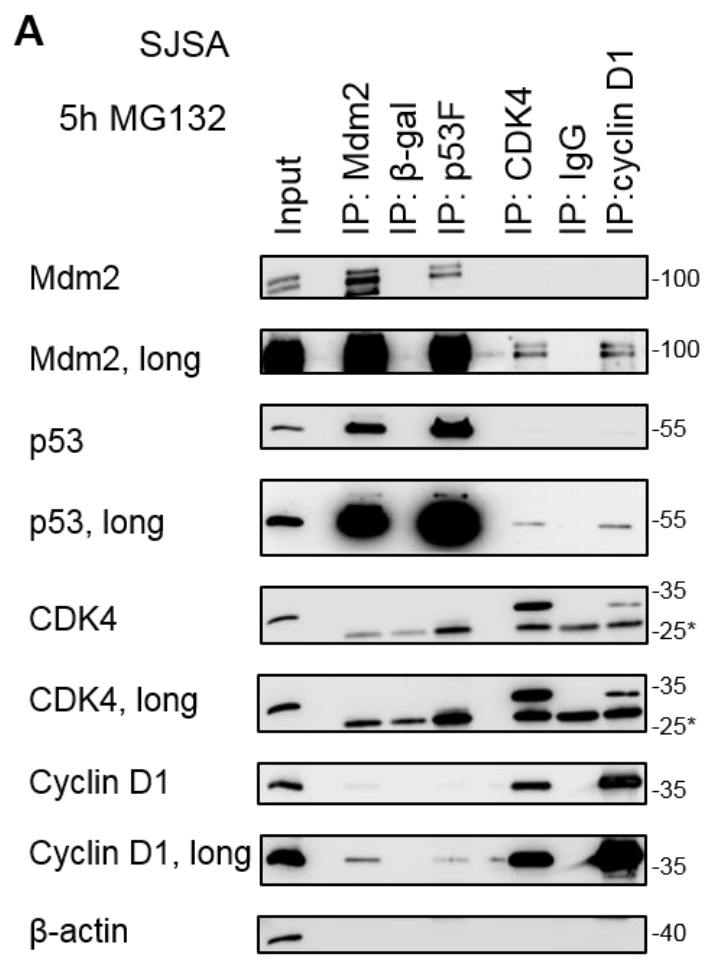

Figure 3.3 5 Association of the CDK4/Cyclin D1 complex and the p53/MDM2 complex.

A. Co-immunoprecipitation of endogenous proteins was carried out with lysates of SJSA cells treated with the proteasome inhibitor 20 $\mu \mathrm{M}$ MG132 for 5 hours. Antibodies to precipitate MDM2 or p53 were compared with an anti-beta-galactosidase antibody as a control, and antibodies to CDK4 and cyclin D1 were compared to pre-immune IgG. Immunoblot analysis of the precipitated material showed association of the CDK4/cyclin D1 complex with the MDM2/p53 complex. Representative figure of three biological repeats. * indicates the immunoglobulin heavy chain IgL

The interaction of p53 and CDK4 was also found in Nutlin-treated cells (Figure 3.3.5B,C). 
B SJSA
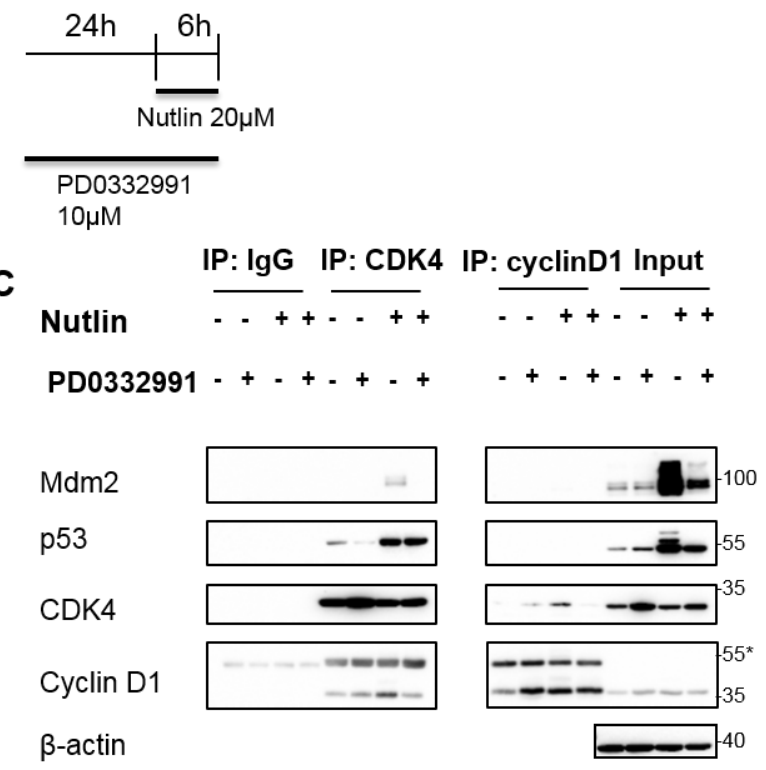

Figure 3.3 5 Association of the CDK4/Cyclin D1 complex and the p53/MDM2 complex.

B. Scheme used for the treatment of SJSA cells with Nutlin and PD0332991.

C. Co-immunoprecipitation was performed from lysates of SJSA cells as described in B. Upon Immunoprecipitation (IP) with antibodies to CDK4, p53 accumulation was observed. When directly precipitating CDK4 or cyclin D1 complex formation between the two decreased upon combined treatment, in comparison to single treatment with Nutlin alone (quantification of the bands in Fig. S3). ${ }^{*}$ indicates the immunoglobulin heavy chain $\mathrm{lgH}$.

Combined inhibition of MDM2 and CDK4 led to decreased association of Cyclin D1 and CDK4 when compared to Nutlin alone (Figure 3.3.5C with quantification in Figure 3.3.S3). Possibly, the CDK4 inhibitor diminishes the proper folding of CDK4 [203] and thus reduces Cyclin D1 binding. The interaction of p53 with Cyclin D1 was further confirmed by plasmid-based overexpression of both components (Figure 3.3.5D). 


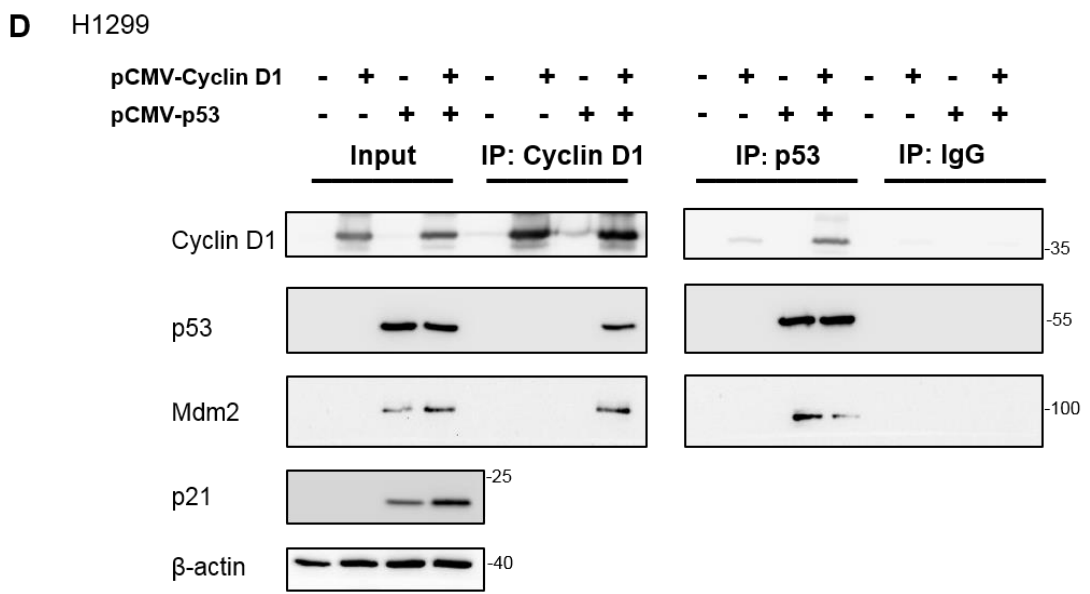

Figure 3.3 5 Association of the CDK4/Cyclin D1 complex and the p53/MDM2 complex.

D. Co-immunoprecipitation was carried out from lysates of $\mathrm{H} 1299$ cells upon plasmid-based overexpression of p53 or cyclinD1, revealing the association of the two, and also the association of MDM2 with p53 in this context.

In sum, p53 physically associates with CDK4 and CyclinD1, suggesting a mechanism by which CDK4 might directly regulate the activity of $\mathrm{p} 53$ as a transcription factor.

\section{CDK4 inhibition does not interfere with p53 binding to its cognate promoter elements but diminishes the recruitment of RNA Polymerase II}

To mechanistically understand how CDK4/6 inhibition reduces the activity of p53 as a transcription factor, we performed immunoblot analysis to detect the acetylation of p53 on Lys 382, an activating modification of p53 [204, 205]. Surprisingly, we observed that the acetylation of p53 was even stronger when PD0332991 was combined with Nutlin than Nutlin alone (Figure 3.3.6A,B). In contrast, we still observed decreased expression of p53 target genes, at the protein (Figure 3.3.6B) and mRNA (Figure 3.3.6C) level, when adding the CDK4/6 inhibitor to Nutlin. 
A SJSA

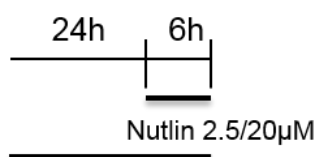

PD0332991

$10 \mu \mathrm{M}$

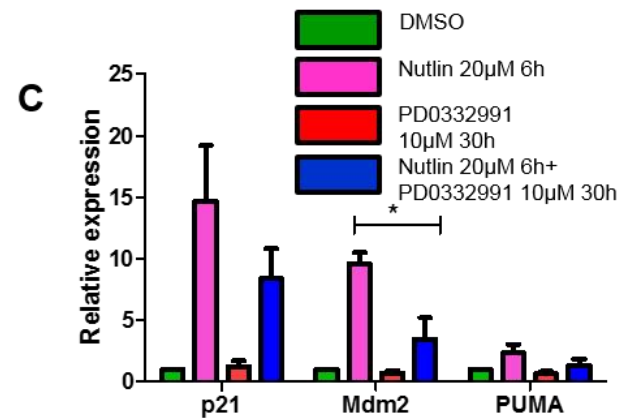

B

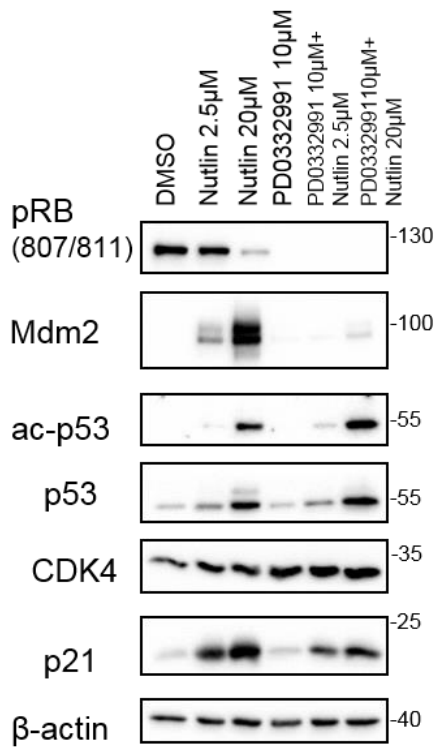

Figure 3.3 6 CDK4 inhibition does not interfere with p53 binding to its cognate promoter elements.

A. Scheme indicating the treatment regimen used for the individual and combinatorial treatment of SJSA cells with Nutlin and/or PD0332991.

B. Immunoblot analysis following treatment as in A. Apart from p53 and its target gene products p21 and MDM2, the acetylation of p53 at Lys 382 was detected. This acetylation was increased, rather than attenuated, upon the combined treatment of Nutlin with PD0332991 in comparison to Nutlin treatment alone.

C. Quantitative Real-Time PCR was carried out to quantify mRNA expression levels of $p 53$ target genes upon treatment as in A, with similar results as in Fig. 2C. Mean of four independent experiments.

To determine differences in nascent pre-mRNA levels, we designed PCR primers spanning exon-intron boundaries of p53-responsive transcripts, namely p21 and MDM2. We observed that CDK4/6 inhibition mostly reduced pre-mRNA levels proximal to the promoters of p53-responsive genes in response to Nutlin (Figure 3.3.6D), arguing that the initiation of transcription depends most strongly on cyclindependent kinases. 

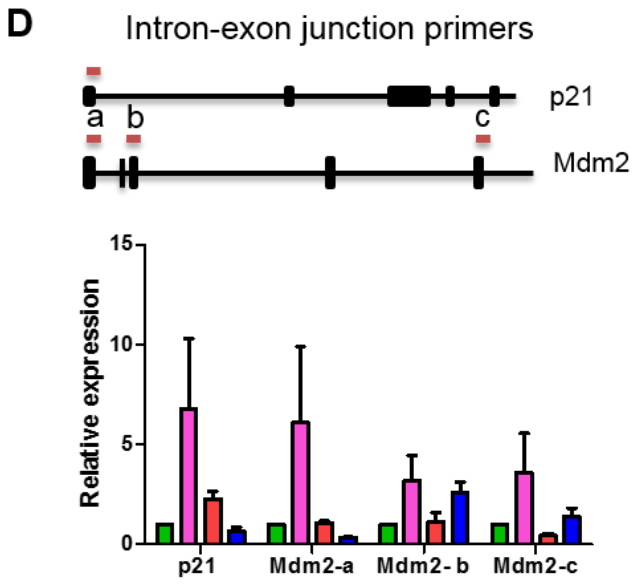

Figure 3.3 6 CDK4 inhibition does not interfere with p53 binding to its cognate promoter elements.

D. Primers spanning Intron-exon junctions were used to quantify pre-mRNA for p21 and MDM2. The origin of the PCR products, with respect of the gene structures, are indicated in the figure. Upon treatment as in A, SJSA cells were subjected to mRNA analysis. It was observed that nascent RNA of p21 and MDM2 was increased upon Nutlin treatment. With the combination, the relative gene expression was reduced, in particular at sites proximal to the promoter.

Finally, we performed chromatin immunoprecipitation (ChIP) analysis to examine p53 occupancy on target gene promoters. Nutlin increased the amount of p53 associated with its cognate promoter elements, as expected. Interestingly, however, CDK4/6 inhibition did not decrease, but even further increased the extent of p53 occupancy on target gene promoters (Figure 3.3.6E), despite the decreased expression of the respective target genes. Similarly, the acetylation of histone H3 at lysine 27 (H3K27ac) at p53-responsive promoters was not impaired, but rather increased (Figure 3.3.6F), still in line with the notion that p53 binding and the subsequent recruitment of histone acetyltransferases [204] is still intact on these promoters. On the other hand, however, the association of RNA Polymerase II with p53responsive genes was decreased in response to CDK4/6 inhibition (Figure 3.3.6G), suggesting that CDK4/6 activity is required for p53-mediated recruitment of RNA Polymerase II. 
$\mathbf{E}$

p53

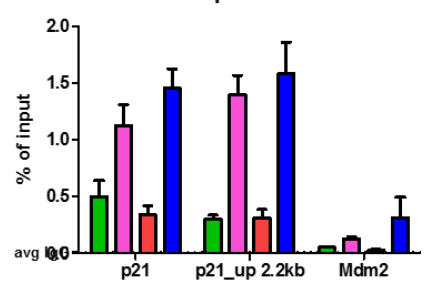

$\mathbf{F}$

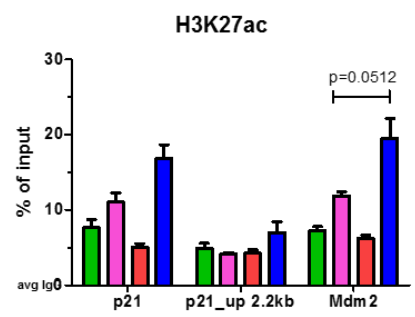

$\mathbf{G}$

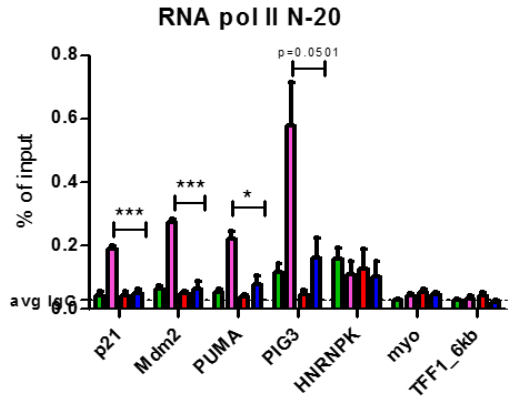

Figure 3.3 6 CDK4 inhibition does not interfere with p53 binding to its cognate promoter elements.

E. Chromatin Immunoprecipitation of p53 was carried out upon treatment with Nutlin and PD0332991 as in A. The occupancy of promoters by p53 remained similar with Nutlin treatment, compared with the combination of Nutlin with PD0332991, at the transcriptional start site (TSS) of p53 target genes p21 and MDM2, and at an enhancer site on p21 which contains another p53 responsive element. IgG was used as a negative control. Mean of four independent experiments.

F. Upon Chromatin immunoprecipitation of histone $\mathrm{H} 3$ with acetylation at $\mathrm{K} 27$, followed by quantitative realtime PCR at the TSSs of p21 and MDM2 as well as the enhancer site of p21, we observed that the enrichment of H3K27ac was increased upon the combination of Nutlin and PD0332991 when compared to Nutlin treatment alone. IgG is used as a negative control. Mean of three biological replicates.

G. Immunoprecipitation for the enzyme pivotal for transcription, RNA Polymerase II. We observed that at the TSSs of the p53 target genes p21, MDM2, PUMA, and PIG3, RNA Polymerase II was enriched with Nutlin treatment. Upon combining this with the CDK4/6 inhibitor, we observed decreased occupancy at the p53 TSS sites. HNRNPK, TFF1_6kb (6kb downstream region of TFF1 gene), and myoD served as negative controls, not associating with the RNA Polymerase II. IgG is used as a negative control as well. Mean of three biological repeats.

This was confirmed by two different antibodies to RNA Polymerase II (Figure 3.3.S4, A-C). In conclusion, CDK4/6 inhibition interferes with the recruitment of RNA Polymerase II by p53, thereby diminishing the initiation of transcription at p53 target genes.

\section{DISCUSSION}

Despite the co-amplification of genes encoding CDK4 and MDM2 in human malignancies, our results indicate that targeting both simultaneously may be counterproductive for cancer therapy. CDK4 inhibition attenuates the p53-response to MDM2-targeted drugs, resulting in decreased cytotoxicity. The CDK4Cyclin D1 complex associates with p53, and its activity is required for gene induction and RNA 
Polymerase II recruitment by p53. Moreover, cell proliferation is diminished in response to Nutlin alone but partially re-established by co-treatment with Nutlin and a CDK4 inhibitor.

We show that the complexes of CDK4 and Cyclin D1 on the one hand, and MDM2 and p53 on the other hand, associate with each other. These findings are in line with a previous report that MDMX, another close binding partner of MDM2 and p53, is phosphorylated by CDK4 [206]. According to this report, CDK4-mediated phosphorylation of MDMX stabilizes its interaction with MDM2 to antagonize p53. This type of regulation would be different from the support of p53 activity by CDK4 reported here. However, since CDK4 needs to associate with MDMX at least temporarily to phosphorylate it, it is conceivable that this interaction further enables the association between the two complexes. This notion is further strengthened by a recent report on a physical association between Cyclin D1 and MDM2 [207]. p53 is a phosphoprotein that undergoes numerous posttranslational modifications. Given that we observed an interaction of CDK4-Cyclin D1 with p53, one might ask whether p53 might be a substrate for the kinase CDK4. However, in vitro kinase assays did not reveal any such direct phosphorylation [208].

How could CDK4 and/or Cyclin D1 assist in the recruitment of RNA Polymerase II to p53-responsive promoters? Previous studies revealed that Cyclin D1 [209, 210] as well as the CDK4-related kinase CDK6 [211] can associate with target genes and function as transcriptional regulators. Interestingly, one of the genome-wide ChIP analysis revealed that Cyclin D1 binds with some preference to sites that also associate with p53, among other transcription factors [212]. These observations further support the idea that CDK4/CyclinD1 might act as a co-factor for p53 in target gene activation.

A previous report is in seeming contradiction with our findings, claiming that Palbociclib cooperates with the MDM2 antagonist Idasanutlin (RG-7388) in killing liposarcoma cells [194]. Despite testing numerous conditions, we were unable to observe a similar synergy. One of the differences might be that in their study, the authors mostly used Idasanutlin concentrations that had little effect on tumor cell growth on their own, thus precluding any possible antagonism to begin with. While we can never rule out that the drugs might cooperate more favorably under clinical conditions, our results do give rise to caution when treating patients. We suggest that the combination of inhibitors to CDK4 and MDM2 in the clinic should be either avoided entirely or otherwise should be used only after carefully balancing the potential benefits with the antagonism reported here.

Another previous report argued that the cytotoxic effects of Nutlin on p53-proficient cells might depend on the ability of MDM2 to degrade the retinoblastoma protein $\mathrm{Rb}$ [197]. In cells where accumulated MDM2 leads to the degradation of hypophosphorylated $\mathrm{Rb}$, apoptosis can be induced, but when $\mathrm{Rb}$ remains, the cells merely arrest. Such a scenario would argue against the efficacy of combining CDK4 inhibition with Nutlin, in line with our observations. CDK4 inhibition can be expected to increase the accumulation of hypophosphorylated Rb, since CDK4 is a key driver of Rb phosphorylation [195]. This, perhaps in addition to the diminished p53 activity reported here, would then result in decreased apoptosis [197]. 
Some conventional chemotherapeutic drugs depend on the activation of p53 for their efficacy. Comparing p53-proficient and -deficient HCT116 cells, such a dependency was found in particular for 5-fluorouracil (5-FU) [213]. On the other hand, the same report showed that p53-deficient cells displayed a higher sensitivity towards topoisomerase inhibitors such as Adriamycin. In analogy, the combination of CDK4 inhibitors and the resulting p53 attenuation would be counterproductive for 5 -FU but might be beneficial for Adriamycin. However, given the additional cell cycle regulatory functions of CDK4 inhibition, each combination remains to be tested individually.

What combinations could be more promising for cancer treatment? Our previous work suggests that the phosphatase PPM1D/Wip1 might represent a suitable target of drugs that synergize with MDM2 antagonists [177, 214]. Wip1 dephosphorylates p53, thereby compromising its activity as a transcription factor. Interfering with this dephosphorylation enhances activating p53 modifications. When this is combined with MDM2 inhibitors, both stability and activity of p53 are increased, leading to pronounced cell death. The DNA-damaging drug Trabectedin, currently used in second line for treating soft tissue sarcoma, was also reported to synergize with the MDM2 inhibitor RG7112 [215], perhaps as a result of p53 accumulation (through MDM2 inhibition) and activating p53 modifications (through DNA damage response). In pre-clinical investigations and cell culture, MDM2 antagonists also cooperated efficiently with MEK or PI3K inhibitors, BH3 mimetics, BCR-ABL antagonists, and HDAC inhibitors [216]. For neuroblastoma cells, MDM2 inhibitors cooperate with ALK inhibition [217]. In the case of CDK4 inhibitors, combination partners are less obvious. In breast cancer treatment, the CDK4/6 inhibitor Palbociclib is often combined with Letrozole, an inhibitor of aromatase in estrogen production, but this only makes sense when treating estrogen-dependent tumors [181]. CDK4 inhibition was reported to antagonize drugs that require entry into mitosis for their efficacy, such as taxanes [218], probably due to inhibited cell cycle progression. On the other hand, CDK4 inhibitors were found to cooperate with inhibitors of signaling kinases, such as MEK and PI3K [218, 219]. The underlying mechanisms for this cooperation, however, remain to be elucidated.

On the other hand, the drug antagonism reported here may be used in a beneficial way. In cases where p53 is mutated in a tumor, p53-activation by chemotherapeutics can be considered irrelevant. In such a scenario, it may be beneficial for normal cells if p53-induced cell death is attenuated. Thus, combining CDK4 inhibitors with DNA damaging chemotherapy might turn out to protect non-cancerous tissue in a patient, giving rise to a potential strategy for avoiding undesired general toxicities.

Under physiological conditions, the CDK4-Cyclin D1 complex is active in cycling cells and stem cells, whereas it is inactive in post-mitotic and terminally differentiated cells [220]. The positive impact of the CDK4-Cyclin D1 complex on p53 activity may thus constitute a greater sensitivity of cycling cells towards p53 activation, as compared to resting cells. Physiologically, this would make sense, since cycling cells are at higher risk for giving rise to cancer, whereas post-mitotic cells are unlikely to resume proliferation anyway, and preserving them despite genotoxic stress might help the organism to survive. Thus, the 
dependence of p53 activity on CDK4 appears as a mechanism to channel tumor suppression on cancerprone, proliferating cells, while sparing differentiated cells despite DNA damage.

\section{MATERIALS AND METHODS}

\section{Cell culture, treatment, siRNA and plasmid transfections}

Human CRL-3043 (93T449) and CRL-3044 (94T778) cell lines were purchased from ATCC. GOT-3 cells were a gift from Pierre Åman, University of Gothenburg, Sweden. SJSA and H1299 cells were obtained from the German Collection of Cell lines (DSMZ, Braunschweig) and maintained in Dulbecco's modified Eagle's medium (DMEM). CRL-3043, CRL-3044, GOT-3 cells were maintained in RPMI medium. Cell culture media was supplemented with $10 \%$ fetal bovine serum (FBS) and antibiotics including penicillin/streptomycin and ciprofloxacin. Cells were maintained at $37^{\circ} \mathrm{C}$ with $5 \% \mathrm{CO}_{2}$. For treatment of cells, Neocarzinostatin (NCS, $0.5 \mathrm{mg} / \mathrm{mL}$, Sigma-Aldrich), Nutlin-3a (Sigma N6287 and BOC life sciences 675576-98-4), Palbociclib (PD0332991 isethionate, Sigma PZ0199), Ribociclib (LEE011, Selleckchem S7440), Abemaciclib (LY2835219, Selleckchem S7158), MG-132 (Calbiochem 474791) were diluted in pre-warmed medium and added to the cells for the indicated periods of time. For siRNA mediated transfection using Lipofectamine 2000 (Life Technologies), cells were reverse transfected with 10nM siRNA to MDM2 (Ambion; custom made, AAGCCAUUGCUUUUGAAGUUAtt (sense), UAACUUCAAAAGCAAUGGCUUtt (antisense)); CDK4 (s2822, Ambion) and a negative control siRNA. Medium was changed after 24 hours and cells were harvested 24 hours later. For plasmid overexpression, $2 \mu \mathrm{g}$ of plasmid was transfected along with Lipofectamine 2000 by forward transfection. Medium was changed after 4 hours and the cells were harvested 24 hours later.

$\begin{array}{ll}\text { Plasmid } & \text { Origin } \\ \text { pCMV6XL5 } & \text { Origene } \\ \text { pCMV-MDM2 } & \text { B. Vogelstein [221] } \\ \text { pCMV-cyclinD1 } & \text { Addgene 19927 }\end{array}$

\section{Quantitative mRNA analysis by qRT-PCR.}

Total RNA was isolated using TRIzol (Invitrogen), followed by cDNA synthesis using Moloney Murine Leukemia Virus reverse transcriptase and random hexamer primers (Thermo Scientific). Home-made SYBR green master mix was used for quantification using real-time PCR. Gene expression levels were normalized to the mRNA from the RPLPO gene and the analysis was conducted using the $\triangle \triangle \mathrm{Ct}$ method. qRT-PCR primer sets were chosen as follows:

\section{Table 1 List of primers used for gene expression studies}

$\begin{array}{ll}\text { RPLPO for } & \text { GATTGGCTACCCAACTGTTG } \\ \text { RPLP0 rev } & \text { CAGGGGCAGCAGCCACAAA }\end{array}$




\begin{tabular}{ll} 
p21/CDKN1A for & TAGGCGGTTGAATGAGAGG \\
p21/CDKN1A rev & AAGTGGGGAGGAGGAAGTAG \\
MDM2 for & TCAGGATTCAGTTTCAGATCAG \\
MDM2 rev & CATTTCCAATAGTCAGCTAAGG \\
PUMA/BBC3 for & GCCAGATTTGTGAGACAAGAGG \\
PUMA/BBC3 rev & CAGGCACCTAATTGGGCTC \\
PIG3/TP53I3 for & GCTTCAAATGGCAGAAAAGC \\
PIG3/TP53I3 rev & GTTCTTGTTGGCCTCCATGT \\
p21/CDKN1A ie for & GTGGCTATTTTGTCCTTGGGC \\
p21/CDKN1A ie rev & TGGCAGATCACATACCCTGTTC \\
MDM2 ie for - a & CGGAGAGTGGAATGATCCCC \\
MDM2 ie rev - a & GCTGGGAACCAGCGATAGAG \\
MDM2 ie for - b & CCACAGATGTTTCATGATTTCCAG \\
MDM2 ie rev - b & AGGTGGTTACAGCACCATCAG \\
MDM2 ie for - c & AGGAGATTTGTTTGGCGTGC \\
MDM2 ie rev - c & GGTGAACTGAAATGTTAGCCCAG \\
\hline
\end{tabular}

\section{Immunoblot analysis}

Cells were harvested in protein lysis buffer (1\% Triton-X 100, $1 \%$ Sodium-deoxycholate, $0.1 \%$ SDS, $150 \mathrm{mM} \mathrm{NaCl}, 10 \mathrm{MM}$ EDTA, $20 \mathrm{mM}$ Tris- $\mathrm{Hcl}$ pH 7.5, 2M Urea). After scraping the cells with the lysis buffer, samples were briefly sonicated for $10 \mathrm{mins}$ at high speed to disrupt DNA-protein complexes. The total amount of protein present in each sample was measured by Pierce BCA Protein assay kit (Thermo Scientific Fisher). Prior to loading the samples on the SDS gels, the samples were boiled with $6 \mathrm{x}$-laemmli at $95^{\circ} \mathrm{C}$ for $5 \mathrm{~min}$. Equal amounts of protein samples were separated by SDS-PAGE. This was followed by wet-transfer using nitrocellulose membrane and finally visualized with the following antibodies- $\gamma \mathrm{H} 2 \mathrm{AX}$ (S139) (9718, Cell Signalling), $\beta$-Actin (ab8227, Abcam), p21 (2947, Cell Signalling), pRb (S807/811) (9308, Cell Signalling), Rb (9309, Cell Signalling), MDM2 (OP 46, Calbiochem), p53 (DO-1, sc-126, Santa Cruz), p53-HRP (DO-1, sc-126, Santa Cruz), CDK4 (ab68266 abcam; DCS-35, sc-23896, Santa Cruz), p53 K382ac ( 2525, Cell Signalling), cyclin D1 (ab134175, Abcam).

\section{Cell proliferation assay (Celigo)}

To determine the proliferation of cells under different treatment conditions, cells were seeded at a density $6^{*} 10^{3}$ cells/well in 24-well plates. They were treated with Palbociclib and or Nutlin with DMSO as the control at the indicated concentrations. Their proliferation capacity was measured using the CeligoTM 
Cytometer (Nexcelom, software version 2.0). Cell confluence of triplicate samples was measured every 24 hours for up to 8 days for each time point.

\section{Cell viability assay}

In order to measure cell viability after drug treatment, the cells were seeded at a density of $2{ }^{*} 10^{4}$ cells/well in 96-well plates having white walls and bottom. These cells were treated with Nutlin and or Palbociclib at the indicated concentrations with the highest concentration of DMSO as a control. To the wells without any treatment, PBS or empty DMEM was added to account for background luminescence. The drugs were incubated for 48 or 72 hours as indicated. Following this, luminescence was measured using the CellTiter-Glo® Luminescence Cell Viability Assay (Promega). The CellTiter-Glo® Reagents were mixed and added in a 1:1 ratio to each well. The solutions were incubated in an orbital shaker for 10 mins to facilitate lysis of the cells. Subsequently, the luciferase signal was measured on a LuminometerDLReady ${ }^{\mathrm{TM}}$ Centro LB 960 reader and the measurements for each condition was processed.

\section{Cell cycle analysis}

To analyse the cell-cycle profile under different treatment conditions, the cells were trypsinized and centrifuged to obtain the cell pellets. To each cell pellet, $100 \%$ ethanol was used for fixation overnight. Following fixation, the cells were washed to allow for rehydration. Finally, for analysing cell cycle analysis, propidium iodide was added and the profiles were obtained using Guava system (Millipore). Three biological replicates were processed for each condition using the same gate settings.

\section{Protein co-immunoprecipitation}

To carry out endogenous co-immunoprecipitation in SJSA cells, individual $15 \mathrm{~cm}$ dishes were used for precipitation with one antibody. The cells were harvested in Co-IP buffer 50mM Tris- $\mathrm{HCl} \mathrm{pH} \mathrm{7.5,300mM}$ $\mathrm{NaCl}, 1 \% \mathrm{NP}-40$ and $0.1 \%$ Sodium deoxycholate) along with protease inhibitors (Roche). The homogenized cell lysates were pre-cleared with Protein G sepharose beads (GE Healthcare). Equal amounts of cell lysates were used for overnight precipitation along with $3 \mu \mathrm{g}$ of each antibody. The following day, the cell lysates were incubated with Protein $G$ sepharose beads for two hours. Subsequently, the samples were washed using the Co-IP buffer and the beads were re-suspended in $6 x$ Laemmli Buffer. These samples were subjected to SDS-PAGE followed by immunoblot analysis. For exogenous Co-IP, cells were transfected in 6 well plates with plasmids 24 hours prior to harvesting. The IP procedure was done as described above, using one well per antibody precipitation.

\section{Chromatin Immunoprecipitation (ChIP)}

Chromatin immunoprecipitation (ChIP) was done according to the protocol published by Denissov and colleagues [222]. Briefly, the cells were fixed in $1.1 \%$ formaldehyde (PFA) for $20 \mathrm{~min}$, and quenched with $0.125 \mathrm{M}$ glycine and lysed in a lysis buffer containing $0.1 \%$ SDS. Sonication was done using a 
Bioruptor Pico device (Diagenode) in Pico Microtubes for 15 cycles. The samples were subjected to incubation with antibody $(2 \mu \mathrm{g})$ and Protein A/G PLUS-Agarose (Santa Cruz) beads overnight. Bead interaction was released via 20 min rotation incubation in $1 \%$ SDS and $0.1 \mathrm{M} \mathrm{NaHCO}_{3}$, and the DNAprotein crosslink was reversed by addition of $0.2 \mathrm{M} \mathrm{NaCl}$ and shaking at $65^{\circ} \mathrm{C}$. DNA was purified by the MinElute PCR Purification Kit (Qiagen) and used for targeted PCR. For IP, the following antibodies were used: p53 (DO-1, sc-126, Santa Cruz), IgG (ab46540, Abcam), H3K27ac (C15410196, Diagenode), RNA Polymerase II (MABI0601, MBL Life Sciences; sc-17798, Santa Cruz; sc-899, Santa Cruz). The following primers were used for targeted ChIP:

\section{Table 2 List of primers used for Chromatin Immunoprecipitation}

For transcription start site amplification

$\begin{array}{ll}\text { P21/CDKN1A for } & \text { CTTTCTGGCCGTCAGGAACA } \\ \text { P21/CDKN1A rev } & \text { CTTCTATGCCAGAGCTCAACATGT } \\ \text { MDM2 for } & \text { TTCAGTGGGCAGGTTGACTC } \\ \text { MDM2 rev } & \text { CCAGCTGGAGACAAGTCAGG } \\ \text { PUMA/BBC3 for } & \text { CCCTGCTCTGGTTTGGTGAG } \\ \text { PUMA/BBC3 rev } & \text { AGTCACTCTGGTGAGGCGAT } \\ \text { PIG3/TP53I3 for } & \text { CCCTGGGTACCTGCATTAAG } \\ \text { PIG3/TP53I3 rev } & \text { TAGCCGTGCACTTTGACAAG } \\ \text { myo for } & \text { CTCATGATGCCCCTTCTTCT } \\ \text { myo rev } & \text { GAAGGCGTCTGAGGACTTAAA } \\ \text { P21/CDKN1A TR for } & \text { CCAGGGCCTTCCTTGTATCTCT } \\ \text { p21/CDKN1A TR rev } & \text { ACATCCCCAGCCGGTTCT } \\ \text { TFF1 6kb for } & \text { CAGGCTTCTCCCTTGATGAAT } \\ \text { TFF1 6kb rev } & \text { ACACCCACCTTCCACAACAC } \\ \text { HNRNPK for } & \text { ATCCGCCCCTGAACGCCCAT } \\ \text { HNRNPK rev } & \text { ACATACCGCTCGGGGCCACT }\end{array}$

For H3K27ac ChIP

$\begin{array}{ll}\text { p21 TSS(K27ac) for } & \text { TCAGGTGAGGAAGGGGATGG } \\ \text { p21 TSS(K27ac) rev } & \text { TGTCGCAAGGATCTGCTGG } \\ \text { MDM2 TSS(K27ac) for } & \text { AGATGGAGCAAGAAGCCGAG } \\ \text { MDM2 TSS(K27ac) rev } & \text { GTACGCACTAATCCGGGGAG } \\ \text { p21_u2.2kb_for } & \text { AGCAGGCTGTGGCTCTGATT } \\ \text { p21_u2.2kb_rev } & \text { CAAAATAGCCACCAGCCTCTTCT }\end{array}$




\section{RNA sequencing (same as [177])}

For RNA-sequencing, the quality of total RNA was determined using the Bioanalyzer 2100 from Agilent Genomics. All samples that were analyzed exhibited an RNA Integrity Number $>8$. Library preparation was carried out using the TruSeq RNA LT SamplePrep Kit (Illumina), starting from $1 \mu \mathrm{g}$ of total RNA. Barcodes for sample preparation were used according to the indications given by the manufacturer. Accurate quantitation of CDNA libraries was performed with the QuantiFluor ${ }^{\mathrm{TM}} \mathrm{dsDNA}$ System (Promega). The size range of final cDNA libraries was determined applying the DNA 1000 chip on the Bioanalyzer 2100. cDNA libraries were amplified and sequenced via cBot and HiSeq 4000 (Illumina; SR, $1 \times 50$ bp, 6 $\mathrm{Gb} / \mathrm{sample}$ ca. 40-50 million reads per sample). Sequence images were transformed using the Illumina software from BaseCaller to bcl files. They were demultiplexed to fastq files with CASAVA (version 1.8.2). The quality check was performed using FastQC (version 0.10.1 Babraham Bioinformatics). Fastq files were mapped to the human reference transcriptome (UCSC hg19) using TopHat gapped-read mapper with very sensitive Bowtie2 settings on Galaxy Platform (Version 0.9). Bowtie 2 (version 2.1.0) (Langmead and Salzberg, 2012). The read counting was performed via HTSeq (38) (version 0.6.0) with the following parameters: -f bam -r pos -s reverse -a 10 -t exon -m union. The count files were subsequently subjected for differential analysis using the DESeq2 Package (39) on R (Bioconductor version 3.2.2). Heatmap was generated using z-score analysis. RNA library preparation and sequencing was done by the Transcriptome Analysis Laboratory (TAL, Göttingen).

\section{Statistical testing (same as [177])}

The statistical testing was performed using Graph Pad Prism 6 software. An unpaired t-test with 95\% confidence was performed with an assumed significance for $p$-values $\leq 5 \%$. Asterisks represent significance. ${ }^{* * *}, p \leq 0.001 ;{ }^{* *}, p \leq 0.01 ;{ }^{*}, p \leq 0.05$.

\section{CONFLICT OF INTEREST}

The authors declare no conflict of interest.

\section{ACKNOWLEDGEMENTS}

RNA-Sequencing data of this study were submitted to the GEO, GSE113369. We thank Pierre Åman, University of Gothenburg, Sweden, for GOT3 cells and the Transcriptome Analysis Laboratory (TAL) of the University Medical Center Göttingen for performing RNA sequencing. Our work was supported by the Else Kröner-Fresenius-Stiftung, the Deutsche Krebshilfe, the Wilhelm Sander-Stiftung, the Deutsche José Carreras Stiftung, and the Deutsche Forschungsgemeinschaft.

\section{AUTHOR CONTRIBUTIONS}

AS and MD conceived the project and designed experiments. ZN and AS carried out the analysis of RNA-sequencing data with supervision by SAJ. AD did the co-immunoprecipitations. AS conducted all other experiments. MD drafted the manuscript. AS and SAJ revised the manuscript. All authors read and approved the manuscript 


\subsubsection{Supplemental figures}

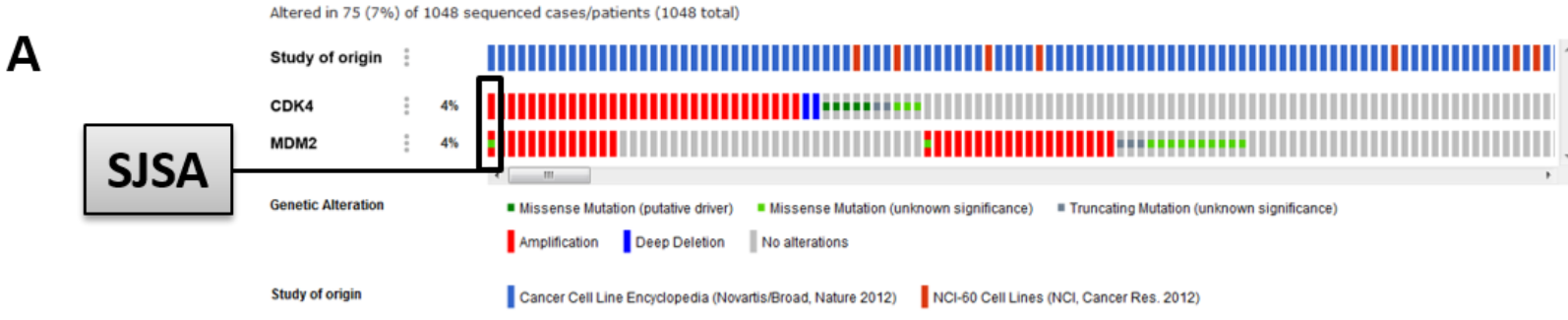

Figure 3.3 S 1 Combined treatment of Nutlin with PD0332991 revealed antagonistic effects with regard to cell viability.

A. Snapshot of the cBioportal dataset from the cancer cell line collections CCLE and $\mathrm{NCl}-60$, with the cell line SJSA containing amplifications of CDK4 and MDM2, according to www.cbioportal.org.

B

$24 \mathrm{~h}$ treatment

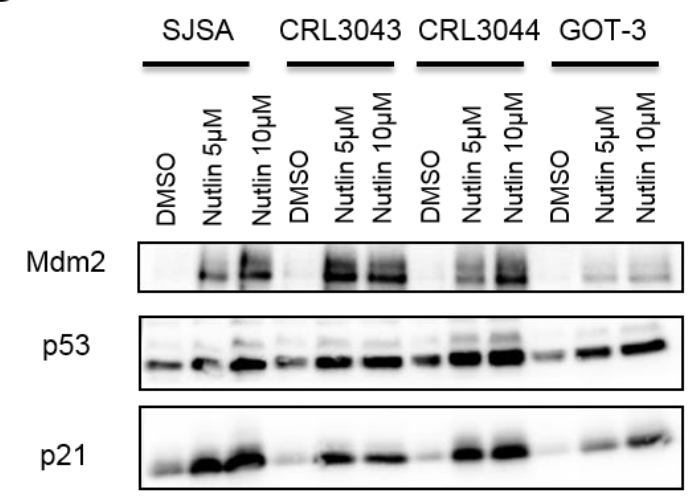

Mdm2

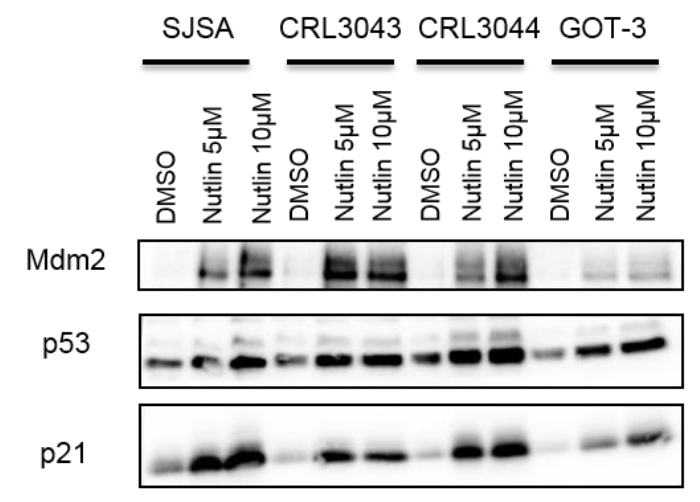

C

$\beta$-actin
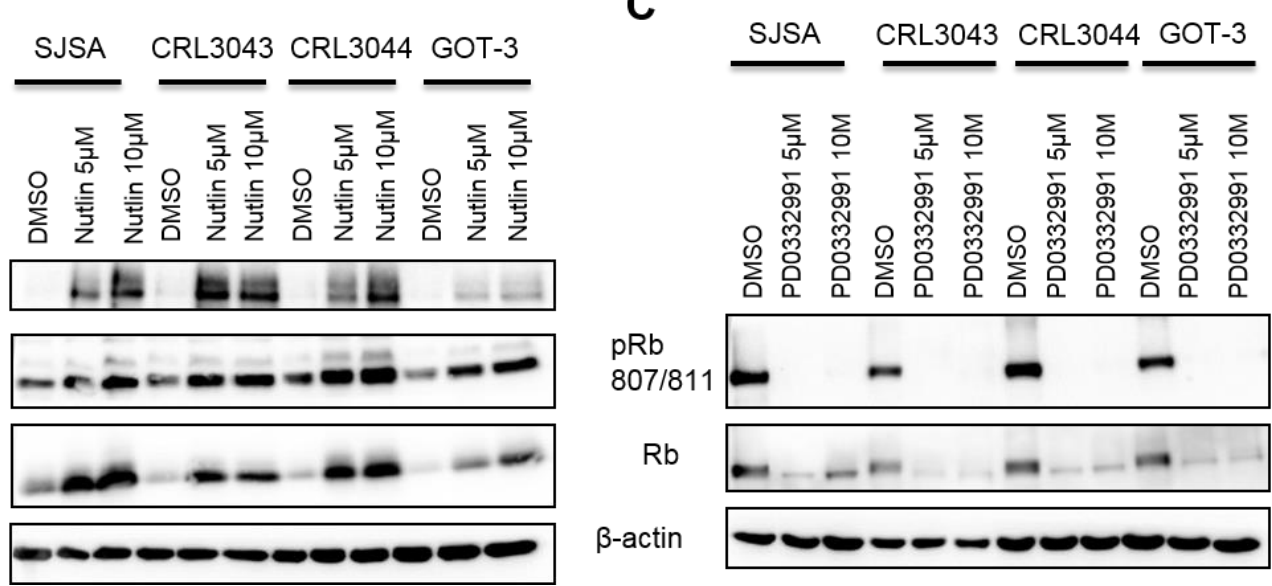

Figure 3.3.S 1 Combined treatment of Nutlin with PD0332991 revealed antagonistic effects with regard to cell viability.

B. SJSA, CRL3043, CRL3044 and GOT-3 cells treated with Nutlin at the indicated concentrations for 24 hours. Lysates were harvested for immunoblot analysis to check for specificity of inhibitor towards its target. Nutlin induced p53 and its target genes - p21 and MDM2.

C. To check for specificity of PD0332991 towards its target - pRb (807/811), SJSA, CRL3043, CRL3044 and GOT-3 cells were treated with PD0332991 at the indicated concentrations for 24 hours. Lysates were harvested and it was noted that PD0332991 reduced phosphorylation on Rb at S807/811. 

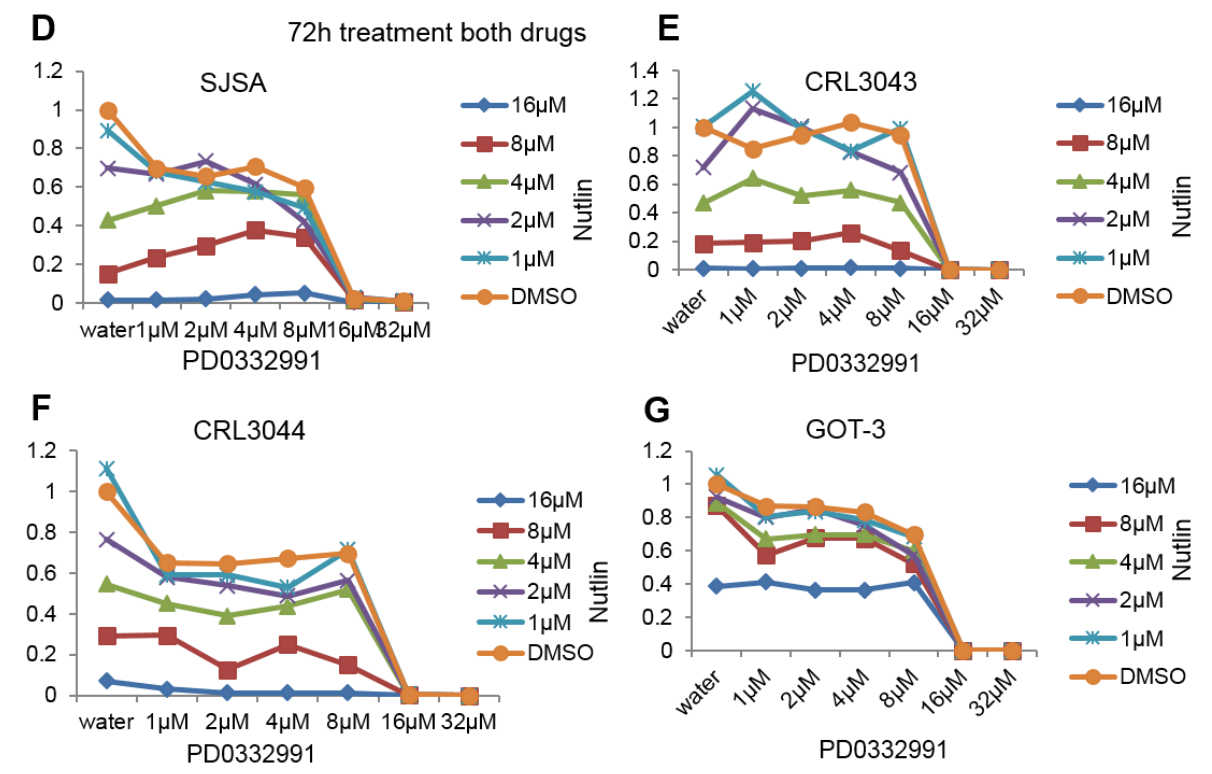

Figure 3.3.S 1 Combined treatment of Nutlin with PD0332991 revealed antagonistic effects with regard to cell viability.

D. Combinatorial treatment with Nutlin and PD0332991 in SJSA cells with increasing concentrations of the two drugs revealed antagonism with respect to cell viability, by luciferase assay to determine ATP levels 72 hours post-treatment.

E. Varying drug concentrations were used to treat CRL3043 cells. Cell viability assays (ATP-luciferase) were performed after 72 hours to reveal antagonistic cytotoxicity of inhibitors to Mdm2 and CDK4/6.

F. CRL3044 cells were treated with increasing concentrations of Nutlin and PD0332991, alone or in combination, followed by viability assays as in $\mathrm{E}$.

G. Viability analysis as in E and F, using another liposarcoma cell line, GOT-3, with similar results. 
A

B

CRL3043

C

CRL3044
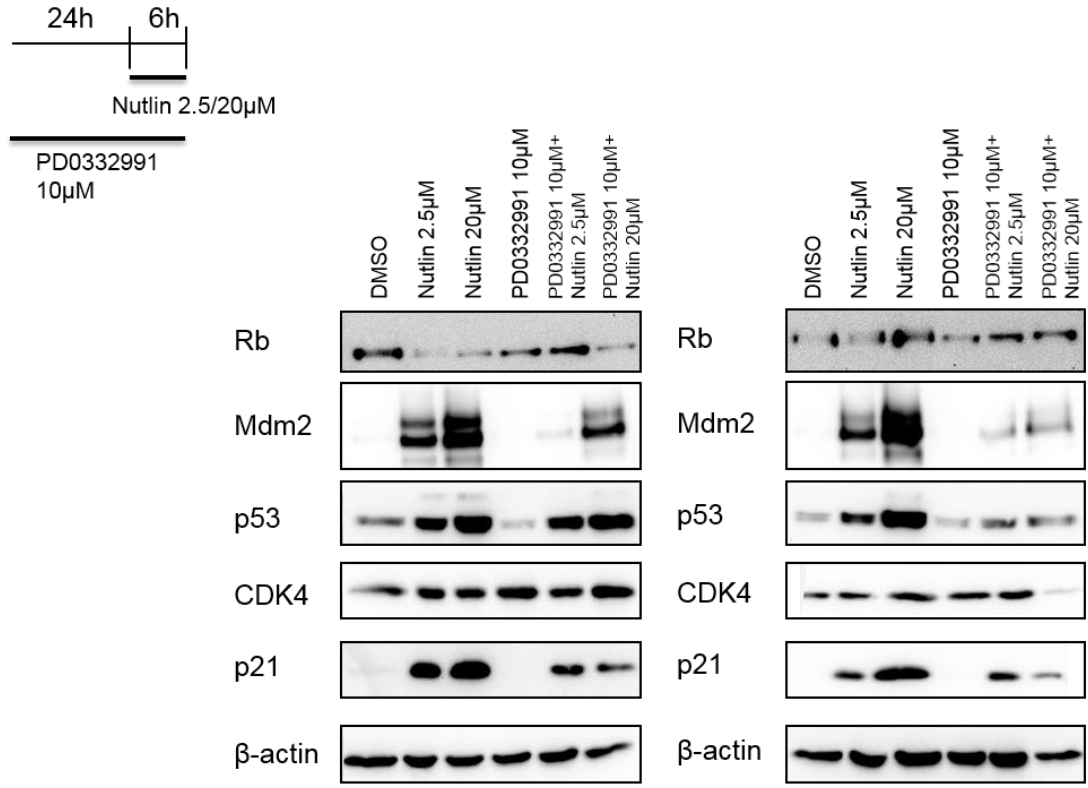

Figure 3.3 S 2. Alternate CDK4/6 inhibitors and MDM2 antagonists decrease p53 target gene expression in various sarcoma cell lines.

A. Treatment scheme is indicated with timings and concentrations of the drugs used.

B. CRL3043 cells (93 T449) were treated with the different drugs and harvested for immunoblot analysis. Upon Nutlin treatment, there was an induction of p53 target genes - p21 and MDM2. This was reduced upon co-treatment with PD0332991. B-actin serves as loading control.

C. CRL3044 cells (94 T778) were treated with Nutlin and PD0332991 at the indicated concentrations and time points. Nutlin indices p53 and its target gene expression namely p21 and MDM2. However, the combination of Nutlin with PD0332991 decreased the expression of its targets.

D SJSA 6 treatment both drugs

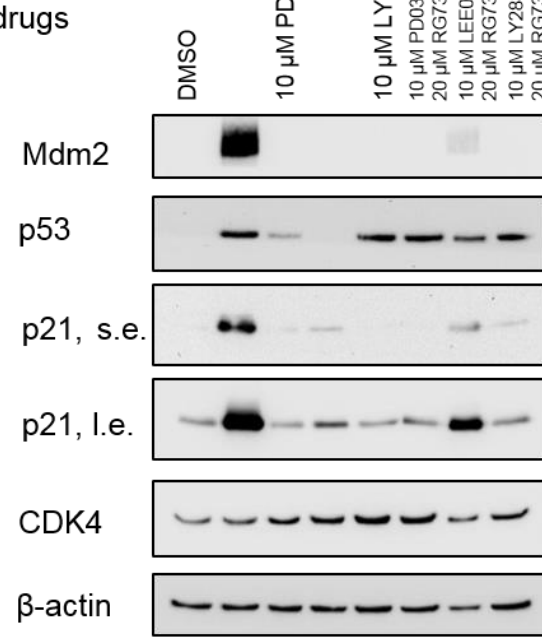

E SJSA

$24 \mathrm{~h}$ treatment

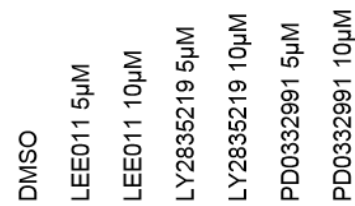

pRb (807/811)

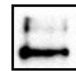

CDK4

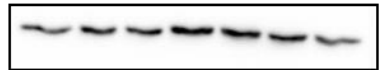

$\beta$-actin

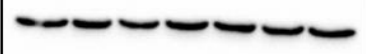


Figure 3.3 S 2 Alternate CDK4/6 inhibitors and MDM2 antagonists decrease p53 target gene expression in various sarcoma cell lines.

D. SJSA cells were treated with RG7388; another MDM2 antagonist alone and in combination with various CDK4/6 inhibitors that are currently FDA approved - LEE011 (Ribociclib) and LY2835219 (Abemaciclib) and PD0332991 (Palbociclib). We again observed that RG7388 treatment accumulates p53 and its target genes while treatment with CDK4/6 inhibitors decreases the activation.

E. SJSA cells treated with various CDK4/6 inhibitors for 24 hours to show that they reduce pRb (807/811), its primary target.

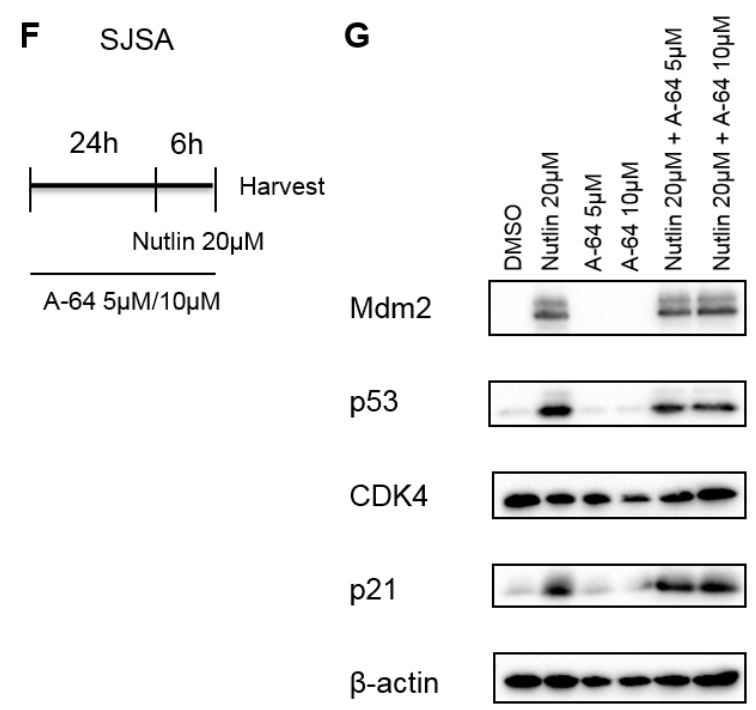

Figure 3.3 S 2 Alternate CDK4/6 inhibitors and MDM2 antagonists decrease p53 target gene expression in various sarcoma cell lines.

F. SJSA cells were treated with Nutlin and the HIPK2 inhibitor A-64 as indicated.

G. SJSA cells were harvested for immunoblot analysis after treatment as in F. Upon Nutlin treatment, p53 as well as MDM2 and p21 proteins accumulated. Their levels remained similar upon co-treatment with the HIPK2 inhibitor A-64.

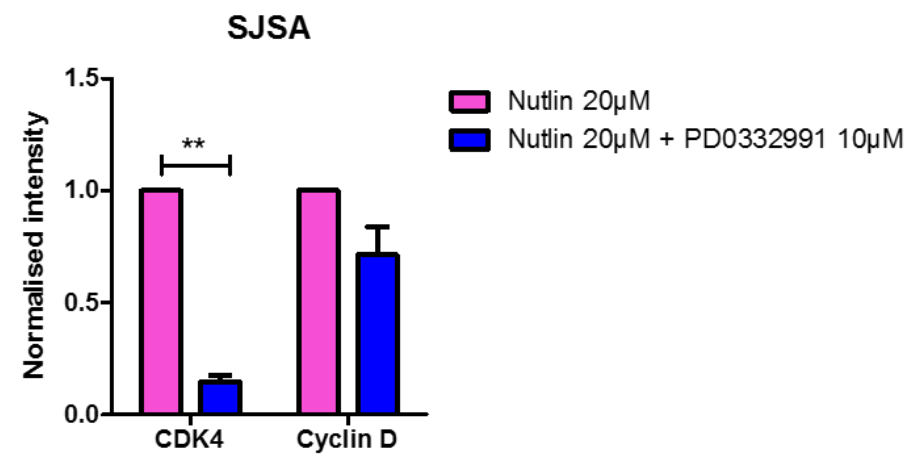


Figure 3.3 S 3. Impaired complex formation of CDK4 with cyclin D1 upon combined inhibition of MDM2 and CDK4/6.

A. Normalized band intensities from Fig. 3.3.5C, corresponding to CDK4 and cyclin D1, upon treatment with Nutlin alone or Nutlin with the CDK4 inhibitor and precipitation with antibodies to Cyclin D1 and CDK4, respectively. The reduction shows diminished interaction upon CDK4 treatment. Mean of three biological experiments.

A SJSA

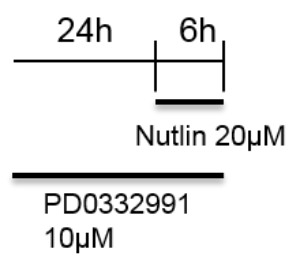

B

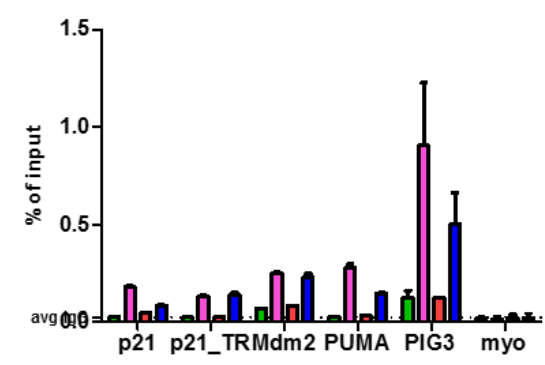

C

RNA pol II A-10

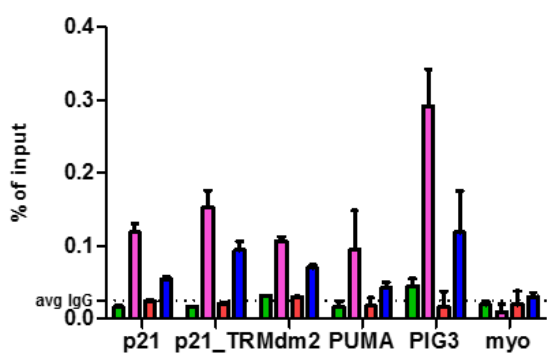

DMSO

Nutlin $20 \mu \mathrm{M} 6 \mathrm{~h}$

PD0332991

$10 \mu \mathrm{M} 30 \mathrm{~h}$

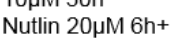

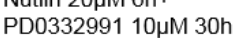

Figure $3.3 \mathrm{~S}$ 4. Alternate RNA polymerase II antibodies indicate decreased occupancy upon combined treatment.

A. SJSA cells were treated with Nutlin and PD0332991 as indicated in the scheme.

B. RNA polymerase enzyme from MBL life sciences was used for the chromatin immunoprecipitation (ChIP). We observed that with Nutlin treatment, there is an enhanced occupancy of RNA polymerase II at the TSS of p53 target genes which is decreased when the cells are co-treated with PD0332991. MyoD serves as a negative site. IgG is a negative control. Mean of two independent experiments.

C. RNA polymerase enzyme from Santa Cruz A-10 was utilized for checking the occupancy with Nutlin and Nutlin and PD0332991 treatments. As observed previously, there was decreased occupancy of RNA pol II with combined treatment suggesting that the decreased p53 target gene expression is due to defect in recruitment of RNA polymerase II enzyme. 
Supplemental tables and RNA-sequencing data have been deposited to the GEO database. The accession number is GSE113369.

References for this section is included along with the introduction and discussion at the end of the thesis. 


\section{DISCUSSION}

In this thesis, we analyzed different strategies for therapeutically targeting p53, a widely studied tumor suppressor, referred to as the 'cellular gatekeeper of growth and division' [223] or 'guardian angel of our genome' [224]. Here, we demonstrate the various outcomes of p53 activation upon combining MDM2 inhibitors with other drugs (Figure 41 )

1) Notably, prior activation of p53 led to the protection of p53-proficient cells against the cytotoxic effects of WEE 1 inhibition and gemcitabine. This protective effect was achieved by p53-mediated cell-cycle arrest carried out by p21.

2) Moreover, pharmacological inhibition of two negative regulators of p53, WIP1 and MDM2 led to increased stability and transactivation of p53 target genes. This enabled the cells to attain a state of pronounced cell-cycle arrest with mild senescent phenotype.

3) Finally, we observed an antagonistic effect on cellular cytotoxicity and p53 activity upon targeting CDK4 and MDM2 in sarcoma cell lines harboring amplifications of the two genes. This study led to the identification of a novel interaction partner of p53, namely the CDK4-cyclin D1 complex. Additionally, the combined inhibition led to the decreased recruitment of RNA polymerase II to the promoters of p53 target genes, thereby elucidating a supportive role of CDK4 in regulating p53 activity.

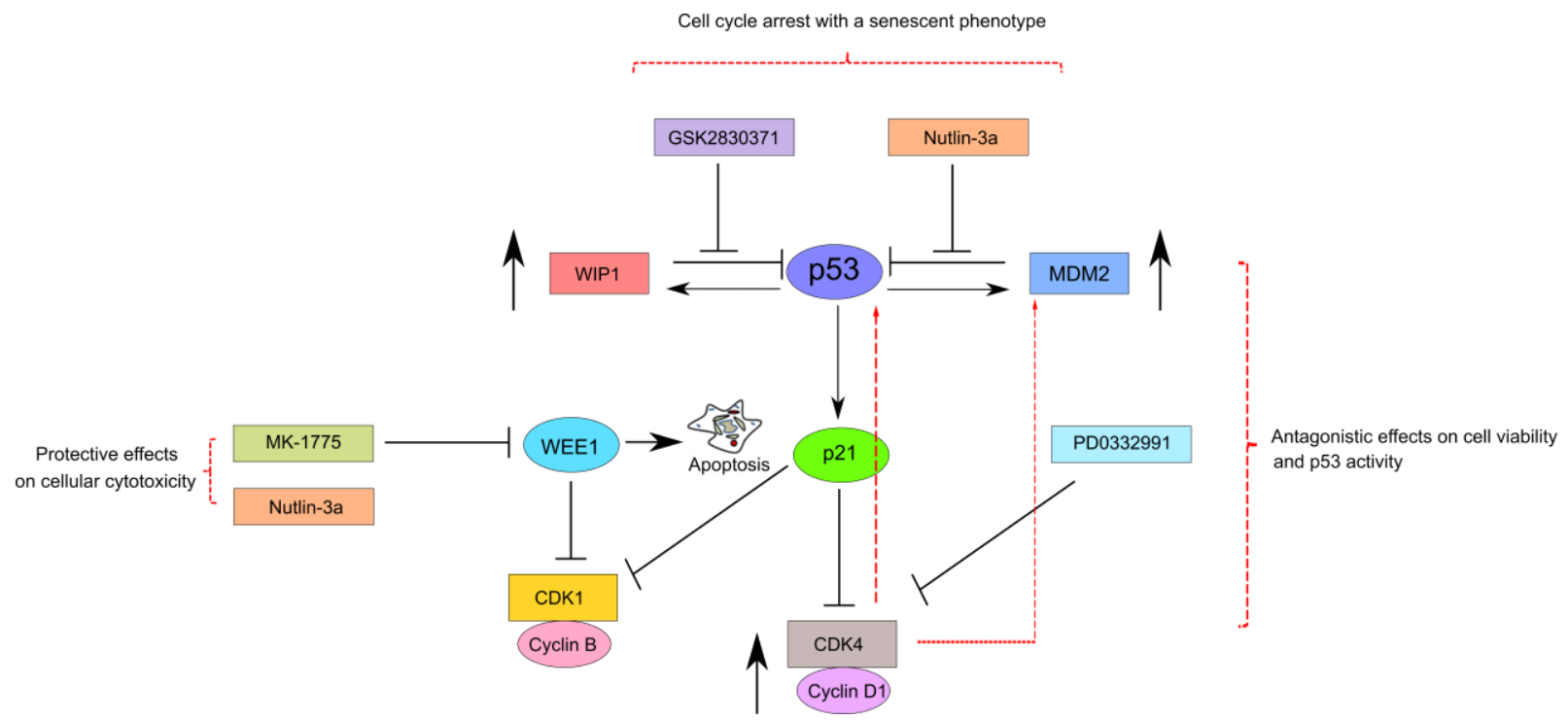

Figure 41 Scheme employed for fortifying p53 activity and the resulting outcomes generated in our study.

Upon combined inhibition of MDM2 and WIP1, we observed a synergistic decrease in cell viability with increased activation of p53 which led to cell-cycle arrest with a mild senescent phenotype. Chemoprotection with MDM2 antagonist, Nutlin was achieved in p53-proficient cells against cytotoxic compounds such as WEE1 inhibition and gemcitabine. Moreover, combined inhibition of MDM2 and CDK4 displayed antagonism with regard to cell viability and p53 transcriptional response. Finally, we also demonstrate a novel interaction partner for the p53-MDM2 complex, namely the CDK4-cyclin D1. Red dotted lines indicate the results generated in our study. 


\section{1 $\quad$ Targeting the tumor suppressor p53}

TP53 gene is inactivated in $50 \%$ of human cancers usually by mutations in its DNA binding domain, or abrogation of its signaling response either by overexpression of its negative regulators - MDM2 and WIP1, downregulation of p14ARF by methylation of the CDKN2A locus, or by degradation of p53 via Human Papillomavirus E6 (HPV-E6) [4, 5, 46, 47, 119, 120, 225, 226]. As mentioned in the introduction, targeting the interaction between p53 and its negative regulator MDM2 using derivatives that bind to MDM2, mimicking three key residues of p53 i.e. Phe19, Trp23 and Leu26 have been studied extensively [166]. A few MDM2 inhibitors were tested in clinics but their efficacies were limited. Moreover, these attempts have not been very successful so far $[167,168]$. Hence, identifying ways to manipulate p53 in a targeted manner for improving its therapeutic potential and efficacy in patients is still of great importance. In this thesis, we suggest three different strategies of using MDM2 inhibitors.

\subsection{Strategies of MDM2 inhibition}

\subsection{1 p53 accumulation protects cells against toxic effects of WEE1 inhibition}

In this study, we demonstrate that pre-accumulated p53 safeguarded p53-proficient cells from the toxic effects that are generally observed upon combined WEE1 inhibition and gemcitabine treatment (Publication 3.1 Figure 2A-D). WEE1 is a Ser/Thr kinase that phosphorylates its substrate CDK1 at Thr14 and Tyr15. The phosphorylated CDK1 prevents the entry of cells into M phase [227]. Exposure to WEE1 inhibitors results in pre-mature mitosis due to lack of phosphorylated CDK1 (active), which allows cells to bypass the G2-M checkpoint of the cell-cycle even with unreplicated DNA, ultimately leading to apoptosis [228]. Due to its cytotoxic effects that lead to myelosuppression, WEE1 inhibitors have not achieved clinical approval so far [229]. On the other hand, gemcitabine is a nucleoside analog that causes replicative stress in cells during S-phase [230]. Here, we demonstrate that by pre-treating cells with MDM2 antagonists, the toxicity of WEE1 inhibitors and gemcitabine can be reduced due to cellcycle arrest caused by p21 via p53 accumulation. This approach of chemoprotection selectively arrests p53-proficient cells at G1-S and G2-M phases of the cell-cycle and reduces the toxicity of these inhibitors.

In agreement with our work, the protective effect of MDM2 inhibition was demonstrated when p53proficient cells were exposed to nucleoside analogues such as gemcitabine and Ara-C but not chemotherapeutic agents like doxorubicin and cisplatin [231]. In this study, accumulation of p53 preserved the cells from replicative stress in S-phase by arresting them at G1 and G2 phases of the cellcycle. This was determined using clonogenic colony formation assays and flow cytometry analysis. Analogous studies using drugs affecting the mitotic spindle, for instance paclitaxel, epothilones $A$ and $B$, and vincristine achieved protection upon doxorubicin pre-treatment. This was possible by induction of G2- arrest that was dependent on p53 and p21 levels [232]. Moreover, normal cells could be protected from the toxic effects of mitotic inhibitors such as paclitaxel by prior treatment with Nutlin [233, 234]. Pre- 
treatment with Nutlin helped the p53-proficient cells to achieve cell-cycle arrest while the p53-deficient cells underwent apoptosis. All these studies indicate a strong dependency on wild-type functional p53 to confer protective effects.

Our study raises concern regarding the schedule for administration of Nutlin, WEE1 inhibitor and gemcitabine. Addition of WEE1 inhibitor and gemcitabine must precede p53 activation to achieve cytotoxicity in tumor cells. The toxic effects of WEE1 inhibition and gemcitabine might be compromised if p53 is already activated. Therefore, this strategy can only be exploited in the case of tumors devoid of normal p53; where MDM2 inhibition would not be able to confer protective effects due to lack of cellcycle arrest mediated by p21 (Publication 3.1 Figure 3A-C). Meanwhile, chemoprotection would be accomplished in normal p53-wild-type cells by using low, non-genotoxic doses of p53 activators (such as MDM2 antagonists). This ensures p53-dependent cell-cycle arrest that protects the normal cells from the toxic effects of chemotherapeutic drugs. This treatment protocol, referred to as cyclotherapy, allows p53-deficient tumors to remain susceptible towards chemotherapy [235].

An important caveat of MDM2 antagonists is adverse side-effects including neutropenia and thrombocytopenia [165]. These side-effects were observed in liposarcoma patients treated with RG7388, an MDM2 antagonist [167]. In vivo studies using xenografts of HCT116 cells indicated that pre-treatment with Nutlin reduced neutropenia when combined with BI-2536, a PLK inhibitor [236]. This justifies great optimism for such studies that utilize chemoprotection to enter clinical trials. Nevertheless, using concentrations of MDM2 antagonists that cause cytostatic rather than cytotoxic effects would be pivotal to eliminate adverse side-effects.

\subsubsection{MDM2 and WIP1 inhibitors in combination are synergistic in activating p53}

Apart from MDM2, WIP1 is another negative regulator of p53. Induced by p53 upon its activation, WIP1 dephosphorylates p53 primarily at Ser15, rendering it less active. Interfering with MDM2 and WIP1 in a panel of cell lines harboring amplified or truncated WIP1 led to a synergistic decrease in cellular proliferation with increased accumulation of phosphorylated (p53PS15) and acetylated p53 (p53K382ac). Both drugs synergistically increased the expression of p53 target genes leading to cellcycle arrest at the G2 phase of the cell-cycle with mild senescence in cells (Publication 3.2 Figure 6).

Two other groups also indicated similar results upon combining MDM2 and WIP1 inhibitors [237, 238]. Our results agree with their reports, including a genome-wide mRNA sequencing indicating augmented expression of p53 target genes (Publication 3.2 Figure 4) upon combined inhibition. We also demonstrated enhanced promoter occupancy of p53 (Publication 3.2 Figure 5) that in part, might be contributed by increased acetylation of p53 at Lys382 (Publication 3.2 Figure 2). Although Pechackova et al observed increased caspase activity indicative of apoptosis which we did not, this could be attributed to differences in the duration and concentration of the drug treatments. In our cell system, we did not 
observe morphological differences in cells that would indicate apoptosis or cleavage of PARP upon immunoblot analysis when treated with WIP1 and MDM2 inhibitors.

Therapeutic benefits of WIP1 inhibition can be maximized by choosing p53-proficient tumors having WIP1 hyper-activation via amplification or truncation of the gene. This would render the combination of the two drugs most sensitive. Although we only observed a mild senescent phenotype with a strong cellcycle arrest, elimination of these tumor cells could be fortified by additional inhibitors which accentuate apoptosis including BH3-mimetics, PI3K and AKT inhibitors. Apart from MDM2 and WIP1 inhibition, treatment with ionizing radiation would enhance ATM-mediated p53 activation at Ser15 which would no longer be counterbalanced by the phosphatase activity of WIP1. This might be a combination that could enhance apoptosis in tumor cells.

\subsubsection{Antagonism of MDM2 and CDK4/6 inhibitors in sarcoma cells}

Clinical trials on liposarcoma patients with MDM2 inhibitors indicated insufficient therapeutic efficiency. The genetic landscape of well-differentiated and de-differentiated liposarcomas is characterized by an amplification of chromosome 12q13-15 region within which resides the gene encoding MDM2, and also CDK4 [239]. An ongoing clinical trial on well-differentiated and de-differentiated liposarcoma patients (NCT02343172) using the MDM2 antagonist HDM-201 and the CDK4/6 inhibitor Ribociclib prompted us to test this combination in a panel of sarcoma cell lines. We noted that the combined inhibition of MDM2 and CDK4/6 led to antagonistic effects on cellular cytotoxicity and p53 transcriptional activity. Interestingly, we were able to demonstrate the interaction of p53-MDM2 and CDK4-cyclin D1 complexes. Furthermore, the combination treatment diminished the interaction of CDK4-cyclin D1 and p53 complexes with decreased recruitment of RNA polymerase II to the target genes of p53. Our study uncovers a novel role of the CDK4-cyclin D1 complex in maintaining p53 activity. This, in turn, provides evidence that the combination of MDM2 and CDK4/6 inhibition is unwarranted in liposarcoma patients due to the lack of synergism with viability and p53 transcriptional activity.

A recent report published by Laroche-Clary and co-workers is contradictory to the effects we have demonstrated [194]. They detect synergism in cell viability and p53 activity upon combining Idasanutlin, an MDM2 antagonist and PD0332991 in one cell line - IB115 derived from a patient sample. Xenograft tumors generated from this cell line indicated reduced tumor burden with increased survival rates upon MDM2 and CDK4/6 inhibition. However, the concentration of MDM2 antagonists used in this study is low, which suggests that their results are mostly due to CDK4/6 inhibition. We tested this combination in not just liposarcoma cell lines, but also in an osteosarcoma cell line (U-2 OS) and non-transformed breast epithelial cells (MCF10A) that indicated antagonism in the p53 transcriptional activity (Figure 5.A1.A-C). This is also suggestive that the attenuated p53 activity is not completely dependent on the amplified MDM2 and CDK4 genes upon their inhibition. Moreover, varying time points and concentrations of CDK4/6 inhibitors in combination with MDM2 inhibitors were tested (Figure 5.A1.D-E). This resulted in 
decreased expression of p53 target genes; even upon combined inhibition for 6 hours. Furthermore, we consistently observed the reduced transcriptional activity of p53 with different MDM2 and CDK4/6 inhibitors (Figure 3.3 2B-C).

In our study, we show that CDK4, a kinase and p53, a protein that undergoes extensive phosphorylations interact with each other (Figure 3.35 ). The immediate question that arises is whether p53 is phosphorylated by CDK4. Our experiments revealed no differences in the phosphorylation of p53 at Ser20 and Ser46 residues upon single treatment with drugs accumulating p53 and in combination with CDK4/6 inhibitor (Figure 5.A2.A-D). This agrees with a study carried out in the lab of Carol Prives using in vitro kinase assays which revealed that the phosphorylation of p53 is mediated by $S$ and G2/M CDKs but not CDK4 or CDK6 [208]. Since CDK2 has been implicated in phosphorylating p53 [240], we depleted the cells of CDK2 (Figure 5.A3.F-G). This did not rescue the downregulated transcriptional response of p53 target genes upon MDM2 and CDK4/6 inhibition. Besides, arresting the cells at the G1 phase of the cell-cycle by CDK4/6 inhibitors excludes the G2/M CDKs. However, we cannot eliminate the off-target effects contributed by CDK4/6 kinase inhibitors utilized in our experiments as they inhibit the ATP-kinase domain [241-243]. Is it possible that CDK4/6 inhibitors are indirectly affecting other kinases that phosphorylate p53? Performing a phosphoproteomic screen of cells treated with MDM2 inhibition alone and in combination with CDK4/6 inhibitors might suggest differences in post-translational modification (PTM) of proteins, in particular of p53 that might explain the transcriptional attenuation.

We observe consistently that the protein levels of MDM2 are more diminished upon combined inhibition of CDK4/6 and MDM2 in various cell lines as compared to the mRNA expression of MDM2. This difference in MDM2 protein and mRNA stability has also been previously described [244]. They suggest that the decreased MDM2 protein levels are post-translationally regulated in part by HAUSP, a deubiquitinating enzyme. In our studies, we observed that upon inhibiting the proteasome using MG132, the MDM2 protein levels were not completely rescued (Figure 5.A3 A-B). Therefore, we hypothesized that another factor regulating the stability of MDM2 is affected upon combined inhibition of MDM2 and CDK4/6. In order to address this, several candidates such as HIPK2, EZH2, p73, and MDMX were tested (Figure 5.A3.C-E; $\mathrm{H}-\mathrm{I}$ ). These proteins were chosen as candidates since they have been described to interact with and regulate the stability of MDM2 [164, 245-247]. However, none of these proteins were found to affect the stability of MDM2 upon their depletion or inhibition.

Contrary to our observations, two groups demonstrated synergistic activation of p53 target genes using pan-CDK inhibitor like roscovitine and a CDK9 inhibitor known as DRB (5,6-dichloro-1- $\beta$-Dribofuranosylbenzimidazole) that can inhibit other kinases including casein kinase II [248, 249]. These drugs were found to synergize with DNA damaging agents like camptothecin and MDM2 antagonists in the induction of p53 activity and stability. This also led to the abrogation of MDM2 levels which they propose could be a result of CDK9 and CDK7 inhibition that in turn affects RNA polymerase II activity. The similarities between these studies and ours are the downregulation of MDM2 protein levels. A follow- 
up experiment that could provide more useful information would be to use a more specific CDK9 inhibitor such as NVP-2 [250] in combination with Nutlin. This would provide further insight into MDM2 stability and p53 activity with CDK9 inhibition in our settings.

Decreased recruitment of RNA polymerase II to p53 target genes upon combined inhibition could suggest a role for $C D K 4 / 6$ to phosphorylate this enzyme or other key transcriptional regulators. RNA polymerase II seemed to be a good candidate since it undergoes extensive phosphorylations at its $\mathrm{C}$-terminal domain (CTD) (reviewed in [251]). Moreover, since CDK9, CDK12, and CDK7 are known kinases that phosphorylate RNA polymerase II [252], it seemed plausible that CDK4/6 kinase might be implicated as well. To test this hypothesis, we looked at total Ser2 and Ser5 phosphorylation sites of RNA polymerase II at specific p53 target gene promoters via chromatin immunoprecipitation (Figure 5.A4 A-C). Although the signals from these experiments upon treatment were not very high, our data does not indicate a role of the kinase activity of CDK4/6 in phosphorylating the CTD of RNA polymerase II at p53 target genes. Are there any other candidates involved in the transcriptional regulation? Transcription factor IIA (TFIIA), a member of the general transcriptional factors (GTF) has been described to be phosphorylated by TBPassociated factor 250 (TAF250) [253]. It will be interesting to check for the occupancy of TFIIA, TAFII250 and Tata-box binding protein (TBP) of the TFIID complex using chromatin immunoprecipitation with the single and double treatments of MDM2 and CDK4/6 inhibitors. This will enable us to have a better understanding of the transcriptional machinery and determine if these general transcription factors are sufficiently recruited to the promoters of p53 target genes.

We displayed the interaction of CDK4-cyclin D complex with p53-MDM2 (Figure 3.3.5A). A study by Yang and colleagues exhibited an interaction between MDM2 and cyclin D1 proteins, and also suggest that the increased cyclin D1 expression following Nutlin treatment is due to increased MDM2 expression [207]. This further supports our data regarding the interaction between these complexes. Furthermore, a recent investigation proposed a novel phosphorylation site at Ser314 on MDMX by CDK4 [206]. They demonstrated that RTK Human Epidermal Receptor growth factor 4 (Her4) inhibits the transcriptional activity of p53 by phosphorylation of MDMX by CDK4 which stabilizes the MDM2/MDMX complex. Although we did not observe increased accumulation of MDM2 in our studies with the combination of MDM2 and CDK4/6 inhibitors, it is conceivable that MDMX and CDK4 probably interact with each other and subsequently phosphorylate MDMX. This agrees with our observation that MDM2 and CDK4 complexes interact with one another. The decreased interaction of CDK4 and cyclin D1 proteins upon addition of CDK4/6 inhibitors could be attributed to differences in protein stability by its displacement from the Cdc37-Hsp90 chaperone complex [203].

As most transcription factors would require co-factor binding, the question arises concerning the possibility of CDK4 or cyclin D1 as a co-factor essential for efficient transcription of p53 target genes. There is currently no literature supporting the role of CDK4 as a transcription factor, although CDK6 has been characterized [254, 255]. Conversely, cyclin D1 is shown to act as a transcriptional regulator [256, 
257], which is suggestive of a putative transcriptional regulatory role in accentuating p53 activity in our study. However, endogenous Chromatin-immunoprecipitation (ChIP) of CDK4 and cyclin D1 did not reveal efficient binding to target genes of p53 which could be due to non-availability of ChIP-grade antibodies, weak binding to the chromatin or less efficiency of ChIP (Figure 5.A5 A-D). Tagged cyclin D1 or CDK4 might help dissect the role of these proteins, if any, in the transcriptional activation of p53 target genes. Interestingly, ChIP sequencing of cyclin D1 in mice showed significant enrichment towards p53 binding sites [257]. Thus, it is possible that cyclin D1 might be an essential regulator of p53 target gene expression. Disrupting the CDK4-cyclin D1 complex with CDK4/6 inhibitors might impair the regulation of these genes. Thus, investigating the possibility of cyclin D1 on the chromatin would provide us with a mechanistic insight in elucidating the antagonistic effects of MDM2 and CDK4/6 inhibition in the p53 transcriptional response. In this regard, using tagged cyclin D1 for pull-down in chromatin immunoprecipitation experiments would provide useful information.

\subsection{Concerns regarding therapeutic inhibition of the MDM2-p53 interaction}

In addition to using MDM2 inhibitors as standalone drugs for the treatment of tumor cells, or xenograft models and patients, identifying potent drug(s) for combination studies is pivotal. Along the same lines, identifying the characteristics of tumors that respond to the combination is principal. In this thesis, we combined MDM2 and WIP1 inhibitors in cells to combat two negative regulators of p53. Two important factors taken into consideration were p53 and WIP1 status of the tumor cells. We used cell lines having wild-type p53 and truncated/amplified WIP1 to maximize the effects of the inhibitors. This led to a strong p53 activation followed by senescence in cells treated with the combination of MDM2 and WIP1 inhibitors [177]. On the other hand, combining MDM2 and CDK4/6 inhibitors in liposarcoma cells that have an amplification of both these genes resulted in drug antagonism with respect to cell viability and p53 transcriptional activity. Consequently, having a clear understanding of the tumor type and drug combination is essential.

Chemotherapy is a commonly used treatment procedure where agents causing DNA damage and impairment of mitosis such as alkylating agents, microtubule and topoisomerase inhibitors, antimetabolites, antifolates and cytotoxic antibiotics [258] are administered to cancer patients. A fascinating study by Bunz and colleagues studied the response of p53 towards different chemotherapeutics namely adriamycin, radiation and 5-fluorouracil (5-FU) using an isogenic pair of colorectal cell lines - HCT116 p53 +/+ and HCT116 p53/- [213]. While DNA damaging agents such as adriamycin and ionizing radiation induced cell death in p53 null cells, these cells were resistant to apoptosis upon 5-FU treatment. Moreover, the tumor responses observed using xenograft models upon treatment with these agents were diminished in comparison to the cellular responses in vitro. Thus, having a clear understanding of the tumor status (p53 wild-type, p53 mutant or p53-null) and type of the treatment is very critical. 
Comprehending the circumstances by which p53 mediates cell death and cell survival in p53-proficient tumors is vital. In cases where p53 remains in its normal state, apart from MDM2 inhibition, induction of apoptosis can be fortified using BH3-mimetics, as reviewed in [214], and death ligands such as tumor necrosis factor-related apoptosis-inducing ligand (TRAIL) [259]. In cases where p53 is non-functional, which occurs in nearly $50 \%$ of human cancers, taking advantage of the situation is paramount. Exploiting the pro-survival functions of p53 in normal cells can be performed by MDM2 inhibition in combination with chemotherapeutics such as gemcitabine, WEE1 inhibitors, cisplatin, actinomycin-D and etoposide [176, 260, 261]. This concept known as 'cyclotherapy' [235] is based on the induction of cell-cycle arrest by CDKN1A expression in normal cells by MDM2 inhibition which helps to repair the damaged DNA that is induced by cytotoxic compounds. In the tumor, however, due to the absence of p53, cells remain cycling. Further addition of chemotherapeutics and genotoxic compounds cause replicative stress that leads to mitotic arrest and accumulation of damage that ultimately results in apoptosis. Applicability of cyclotherapy in clinics still requires further studies. Moreover, the impact of pre-activated p53 in combination with chemotherapeutics on non-proliferating cells such as cardiomyocytes or neurons needs additional testing.

Clinical trials and in vivo studies using MDM2 inhibitors indicated the emergence of p53 mutations in patients [165, 262]. There is a significant tendency of mutual exclusivity in overexpression of MDM2/MDMX and mutations in p53. Cancers where these mutual exclusivities are often observed include liposarcoma, glioblastoma multiforme, melanoma, prostate, Estrogen Receptor -positive (ER) breast cancer and bladder cancer. Therefore, treating such patients with MDM2 antagonists might be a good starting point as a precaution for the emergence of p53 mutations.

Of prime concern were hematological and gastrointestinal toxicities that patients administered with MDM2 antagonists faced which were dose- limiting [167, 263]. The major question that needs to be addressed is how p53 can be targeted in a focused fashion to avoid these adverse effects. Remarkably, in xenograft models upon pre-treatment with Nutlin followed by BI-2536, an inhibitor of PLK1, reduced neutropenia was observed [236]. Currently, different MDM2 inhibitors are in clinical trials. Few of them include, AMG-232 [264] for patients diagnosed with glioblastoma and soft tissue sarcoma, DS-3032b for hematological malignancies [265], and HDM201 in the case of solid tumors [266]. Some others include R06839921; a pegylated form of Idasanutlin which is a prodrug formulated for intravenous administration for treating Acute Myeloid Leukemia (AML) patients (NCT02098967), and APG-115; an analogue of SAR405838 that is used in patients diagnosed with advanced solid tumors and lymphomas. The outcome of these studies might unravel drugs having lower toxicity and reduced adverse effects with increased efficacy for treatment of patients.

\subsection{Alternatives to the classical MDM2-p53 inhibitors}

Having mentioned the concerns regarding the usage of MDM2 inhibitors to activate p53, the question emerges relating to alternatives. Similar to MDM2 inhibition leading to cell-cycle arrest via p21 due to 
p53 activation, CDK4/6 inhibitors such as Palbociclib, Ribociclib and Abemaciclib also cause cell-cycle arrest. This is carried out by inhibiting the phosphorylation of Rb by CDK4/6 which prevents E2F1 to transcribe its target genes that allows cells to progress into the S-phase of the cell-cycle. Therefore, the addition of these drugs would arrest both, the normal cells and tumor cells. Furthermore, combining them with cytotoxic compounds would not be targeted towards a selected population of cells. Hence, it might not represent a viable strategy for treating p53-proficient cancers.

Nonetheless, CDK4/6 inhibitors might be beneficial for the treatment of $\mathrm{Rb}$ negative or Human Papillomavirus E7 (HPV-E7) positive tumors in combination with S or M-phase poisons. Since Rbmediated cell-cycle arrest would be circumvented in the tumor cells upon addition of CDK4/6 inhibitors, they would be most sensitive towards the toxic effects of DNA damaging agents and chemotherapeutics. On the other hand, normal cells that retain functional $R B$ gene will undergo cell-cycle arrest, thus protecting them from the cytotoxic impact of these poisons.

Apart from the plethora of drugs that inhibit the binding of p53 and MDM2 like Nutlins, few compounds are available in the market which are directed towards the C-terminus RING domain of MDM2. These drugs JNJ-26854165 [267], HLI98 [170], MPD [268], MEL 22/23 [169] and others inhibit the ubiquitin ligase activity of MDM2. Treatment of p53-proficient cells with these inhibitors resulted in decreased degradation of p53 by MDM2, along with cell-cycle arrest or apoptosis-related gene expression. Our group showed that the interaction of MDM2 with Polycomb group (PcG) member PRC2 complex leads to the enhancement of repressive marks via H3K27me3 and H2AK119ub on Hox genes that are involved in maintaining stemness [164]. The resulting fate of cells upon targeting both, the N-terminal p53-binding domain and C-terminal RING-finger domain of MDM2 using these drugs might be of interest. Combination of these drugs with WIP1 inhibitors, ionizing radiation, DNA damaging agents, and chemotherapeutic drugs causing replicative stress would be extremely interesting to study.

Thus, simultaneously targeting the ubiquitin ligase function of MDM2 along with activation of p53 will be of utmost interest to clinics. It would also be appealing to study the differentially regulated genes when MDM2 is inhibited at both $\mathrm{N}$ and $\mathrm{C}$-terminal domains in a p53 proficient background. This might provide further insight into the role of MDM2 - is its purpose primarily p53-dependent?, is there a balance between its chromatin-bound RING-finger domain effects and p53-dependent functions? or would the scale shift towards its E3 ubiquitin ligase functions?

\subsection{Harnessing mutant p53}

$50 \%$ of tumors have a mutated p53 protein [4, 5]. An appealing approach enabling p53 to carry out its tumor suppressive functions is to identify therapeutic agents that allow mutant p53 to regain its wild-type p53 functions. PRIMA-1 (p53-reactivation and induction of massive apoptosis) was recently demonstrated to re-activate p53-dependent apoptosis via NOXA in esophageal squamous cell carcinoma cells having missense mutations in TP53 [269]. It acts upon conversion to methylene 
quinuclidinone which binds covalently to cysteine residues in p53 that is sufficient to re-activate the protein [270]. In-silico predictions have suggested multiple peptides that enable reactivation of mutant p53, including proof-of-principle strategies [271-273]. APR-246, a p53-mutant-reactivator and methylated form of PRIMA-1 [173] showed a moderate response in p53-mutated patients diagnosed with Acute Myeloid Leukemia (AML) and Chronic Lymphoid Leukemia (CLL) [274] that is under clinical trials. A fraction of mutant p53 has been described to form aggregates [275] which when targeted restores wildtype p53 functions in a xenograft model using small peptide inhibitor ReACp53 [276]. The therapeutic effects of these inhibitors need to be studied in the clinics.

Apart from reactivating mutant p53, inhibiting it seems to depict an alternative route since tumors often get addicted to mutant p53 [277]. Destabilizing mutant p53 has been extensively exhibited using indirect inhibitors of HSP90, HDAC and SIRT1 [278]. Thus, targeting mutant p53 is a strategy that needs to be extensively examined. This might enable us to treat a wider set of patients that could translate to a better prognosis.

\subsection{Concluding remarks and future perspectives}

In this thesis, we describe strategies of targeting the MDM2-p53 interaction using small-molecule inhibitors that antagonize MDM2, an E3 ubiquitin ligase which causes proteasomal degradation of p53.

Using precise combinations of drugs for the treatment of individual tumors is crucial. Understanding the cancer cell-type, its genetic landscape, and sensitivity of the tumors to each drug is indicative of the response and efficacy of treatment. We observed that MDM2 inhibition alone was not sufficient to increase p53 activation to eliminate tumors. Thus, we performed combination studies by choosing cell lines that would be most sensitive towards these drugs.

Interestingly, we found that inhibiting the function of proteins that are oncogenic in tumor cell lines by its amplification using drugs is not a determinant of its sensitivity. On the one hand, we observed synergistic effects of decreased cell survival and increased p53 activation upon combined MDM2 and WIP1 inhibition in WIP1 amplified/truncated tumors. On the other hand, we observed antagonistic effects on cell viability and p53 response in tumors with amplified $M D M 2$ and $C D K 4$ in response to inhibitors of both these genes. Thus, the combination of inhibitors needs prior testing in cell lines to ensure that drugs which indicate synergism proceed to clinical trials.

Although we observed an unexpected effect upon combining MDM2 and CDK4/6 inhibitors, this led us to uncover a novel role of CDK4 in regulating p53 transcriptional activity. Despite almost four decades of work on the tumor suppressor TP53, we are still deciphering its numerous functions. We could demonstrate a new interacting partner for the MDM2-p53 complex, namely CDK4-cyclin D1. The interaction between CDK4 and cyclin D1 was weakened upon CDK4/6 and MDM2 inhibition; which might indicate a necessity of the complex for proper p53 activation. 
Consequently, having a clear understanding of the pathways regulating p53 transcription is critical for therapeutic benefits. To put this study in perspective, actively dividing cells require CDK4-cyclin D1. In such cases, p53 is required to mediate repair or arrest of the cells in case of damage. However, in postmitotic, senescent or differentiated cells, activation of p53 is not critical since these cells would not accumulate mutations. Therefore, this might be a strategy that cells exploit to keep p53 active in proliferating cells.

p53 plays a key role in myocardial infarction (MI) by inducing apoptosis at the infarcted myocardial tissue [279]. Targeted deletion of p53 in mice with ligated coronary artery to mimic Ml was found to improve their survival rates. Since we observe decreased transcriptional activation of p53 upon CDK4/6 inhibition, it is possible that upon treatment of mice with these inhibitors, apoptosis at the site of the infarcted tissue could be reduced. This would also be helpful in the case of reducing hypoxia-induced endothelial cell death during stroke since it is a p53-dependent process [280]. Moreover, cells lacking p53 have longer survival rates during hypoxia indicating that targeting p53 under such conditions would be beneficial [281]. Thus, it makes it conceivable that CDK4/6 inhibitors can be utilized as p53 inhibitors, which would help to extend the survival rates of patients diagnosed with such conditions.

Finally, we demonstrate a scenario where p53-deficient tumors can be eliminated by cytotoxic drugs such as WEE1 inhibitors and nucleoside analogs like gemcitabine while conferring a protective effect on the p53-proficient cells by prior activation of p53 using MDM2 antagonists. Exploiting cyclotherapy might be beneficial for the treatment of tumor patients having p53-null or mutant tumors. Moreover, CDK4/6 inhibitors might be better drugs for achieving chemoprotection in normal cells due to the reduced adverse effects of myelosuppression observed in patients [282]. This is due to reversible cell-cycle arrest but not apoptosis of the bone marrow cells. Protecting these cells from chemotherapeutics and damaging agents is also possible if the tumors are deficient for $R B$. Thus, exploring this avenue further would be interesting. 


\section{APPENDIX}

A

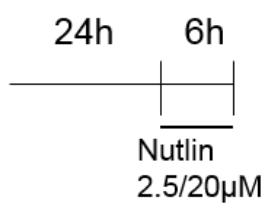

PD0332991

$10 \mu \mathrm{M}$
B

U2OS

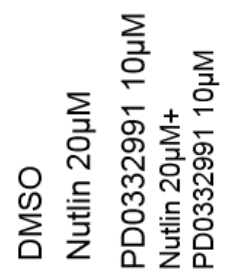

MDM2

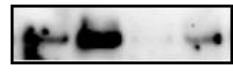

p53

p21
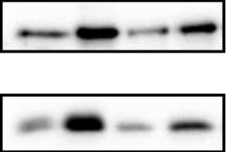

$\beta$-actin

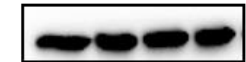

C

MCF10A

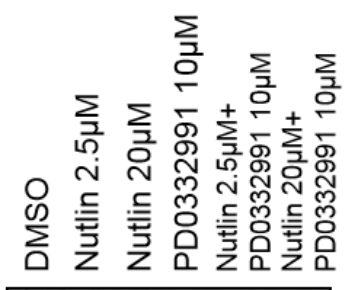

MDM2

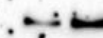

p53

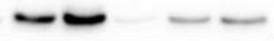

p21

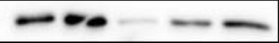

$\beta$-actin

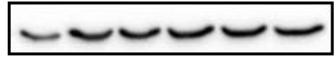

Figure 5 A 1 Alternate cell lines also show diminished activation of p53 when combined with CDK4/6 inhibitor.

A. Scheme denoting the treatment scheme.

B. U-2 OS (osteosarcoma) cells were exposed to DMSO, Nutlin, PD0332991 and the combination treatment. Cells were harvested for immunoblot analysis to check for the expression levels of MDM2, p53 and p21. In both cell lines, treatment of Nutlin with PD0332991 reduced the protein levels of p53 and its target genes p21 and MDM2 in comparison to Nutlin treatment alone. $\beta$ actin serves as loading control.

C. MCF10A (non-tumorigenic breast epithelial) cells were treated with DMSO, Nutlin and PD0332991 at different concentrations. The cells were harvested for immunoblot analysis to check for the expression of p53 target genes such as p21 and MDM2. $\beta$-actin serves as loading control. 


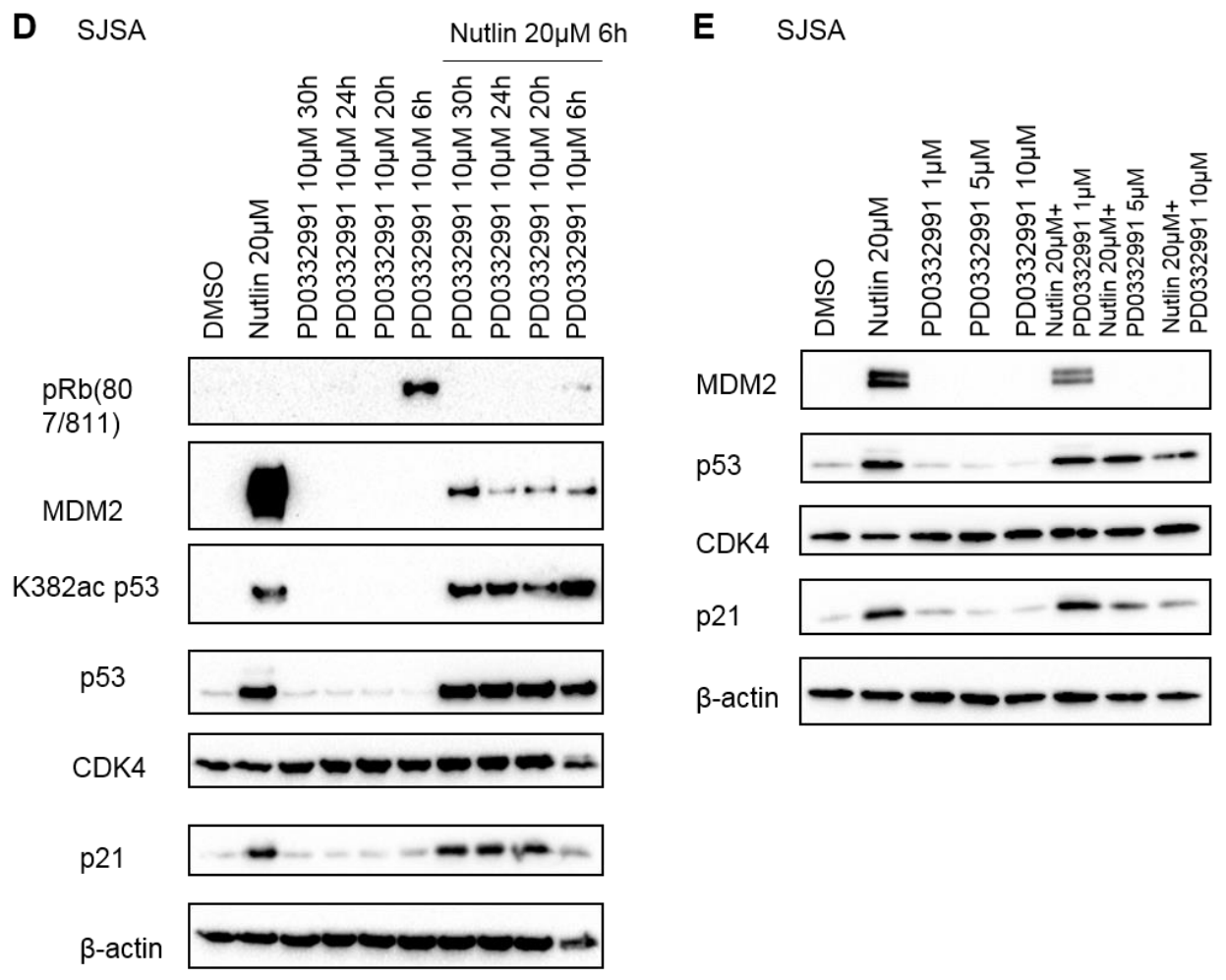

Figure 5 A 1 Various concentrations and time points of cells treated with PD0332991.

D. SJSA cells were treated with PD0332991 at varying time points as mentioned. Immunoblot analysis revealed diminished MDM2 expression even upon 6h combined treatment with Nutlin.

E. SJSA cells were treated with different concentrations of CDK4/6 inhibitor and the cell lysates were assessed for p53 target genes- MDM2 and p21 using immunoblot analysis. We noted decreased MDM2 levels even with $1 \mu$ M PD0332991 in combination with Nutlin. $\beta$-actin serves as loading control. 
A CRL3044

$6 \mathrm{hr}$
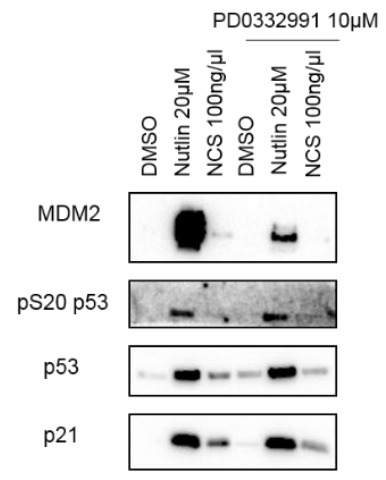

YH2AX

$\beta$-actin

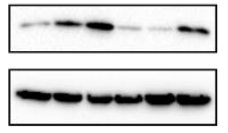

C

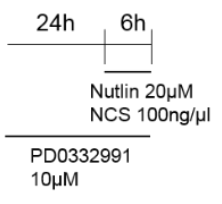

B SJSA

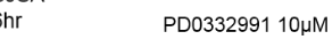

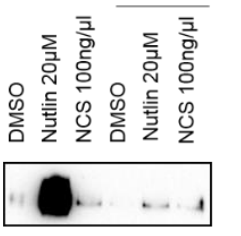

pS20 p53 $\quad \ldots \ldots \ldots$

p53

p21

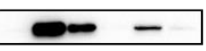

YH2AX

$\beta$-actin

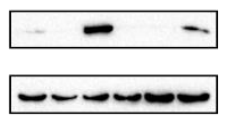

D

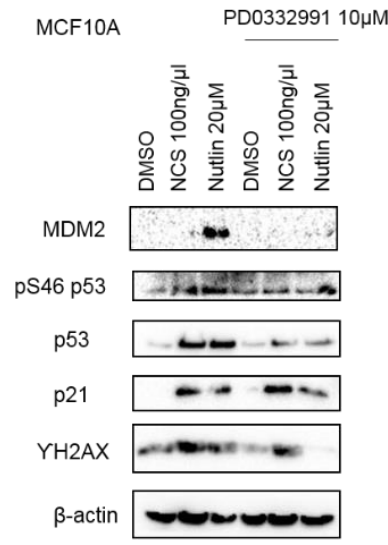

Figure 5 A 2 Phosphorylation of p53 at S20 and S46 upon p53 accumulation and CDK4/6 inhibition.

A. CRL3044 cells were treated with DMSO, Nutlin, NCS alone and in combination with PD0332991 for 6 hours. Immunoblots depicting p53 and its target gene expression shows attenuated response upon combined Nutlin/NCS treatment with PD0332991. pS20 p53, a site phosphorylated upon p53 activation remained unchanged upon Nutlin treatment alone or in the combination.

B. SJSA cells were treated with DMSO, NCS, Nutlin, PD0332991 with the combinations for 6 hours and the cell lysates were harvested for immunoblot analysis to check for p53 activation and stabilization. Again, there were no differences observed in the phosphorylation of p53 at S20 with Nutlin treatment alone or in combination with PD0332991 treatment.

C. Treatment scheme for MCF10A cells.

D. To investigate changes, if any in the pS46 p53 upon Nutlin/NCS treatment alone or in combination with PD0332991, cells were harvested for immunoblot analysis. Probing for pS46 p53 did not indicate strong differences, although we still noted the decreased activation of p53 response. $\beta$-actin serves as loading control. 
A SJSA

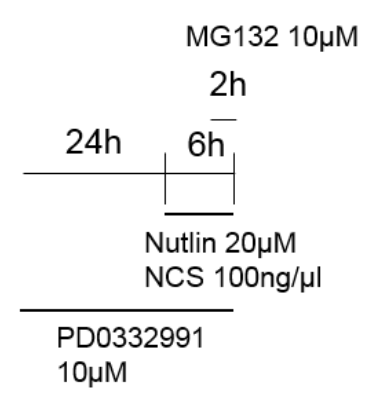

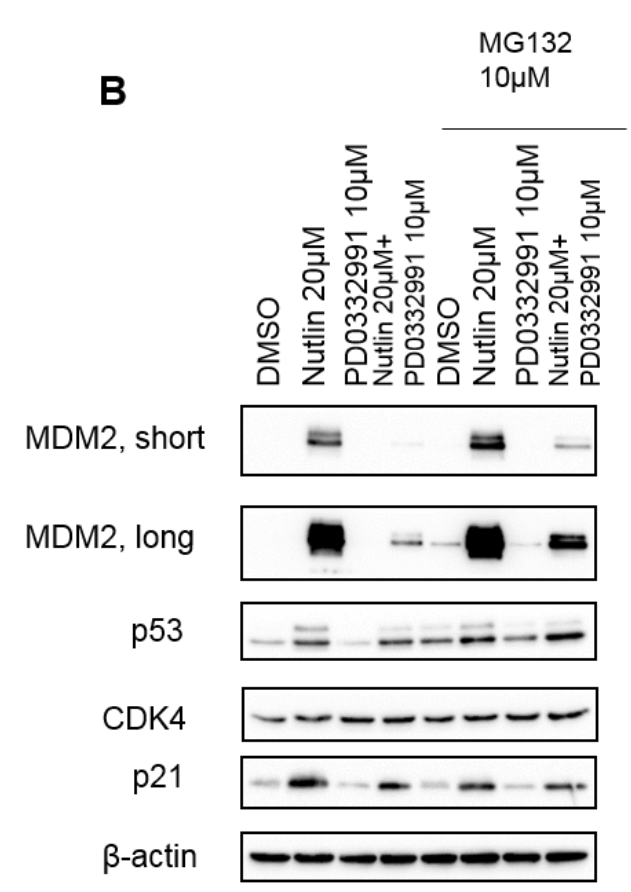

Figure 5 A 3 Treatment with proteasome inhibitor partly rescued MDM2 protein expression.

A. Scheme used for treatment protocol.

B. SJSA cells were probed for p53 and its target genes upon addition of MG132, an inhibitor of the proteasome to check whether the stability of the protein is affected. Immunoblot analysis revealed only partial rescue of MDM2 protein levels upon combined addition of Nutlin and PD0332991 with MG132 in comparison to Nutlin and PD0332991 treatment alone. $\beta$-actin serves as loading control. 
C SJSA

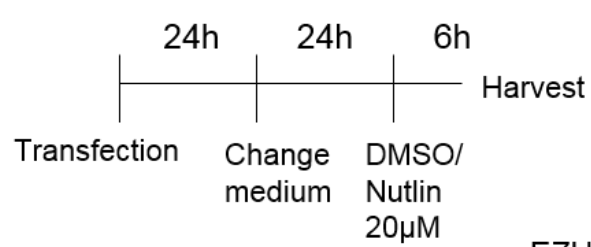

D

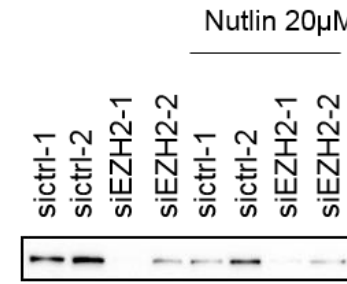

MDM2

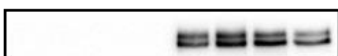

p53

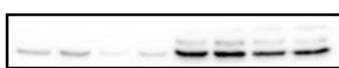

CDK4

p21

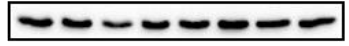

$\beta$-actin
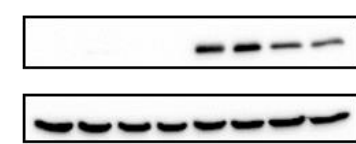

E

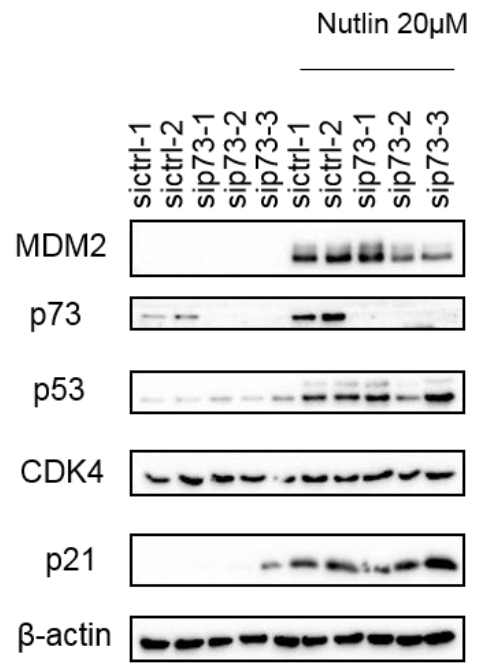

Figure 5 A 3 MDM2 stability and expression remains largely unaffected upon depletion of EZH2 or p73.

C. Treatment protocol.

D. SJSA cells were depleted of EZH2 using two different siRNAs. We observe very slight decrease in protein stability of MDM2 with siEZH2-2 but not siEZH2-1 upon Nutlin treatment.

E. SJSA cells were transfected with three siRNAs towards p73. Two siRNAs - sip73-2 and sip733 decreased the expression of MDM2 in combination with Nutlin treatment. However, the p53 and p21 levels rather increased with sip73-3. $\beta$-actin serves as loading control. 


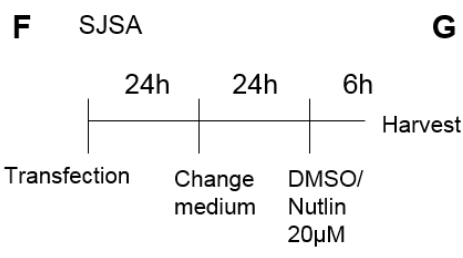

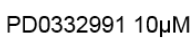

G

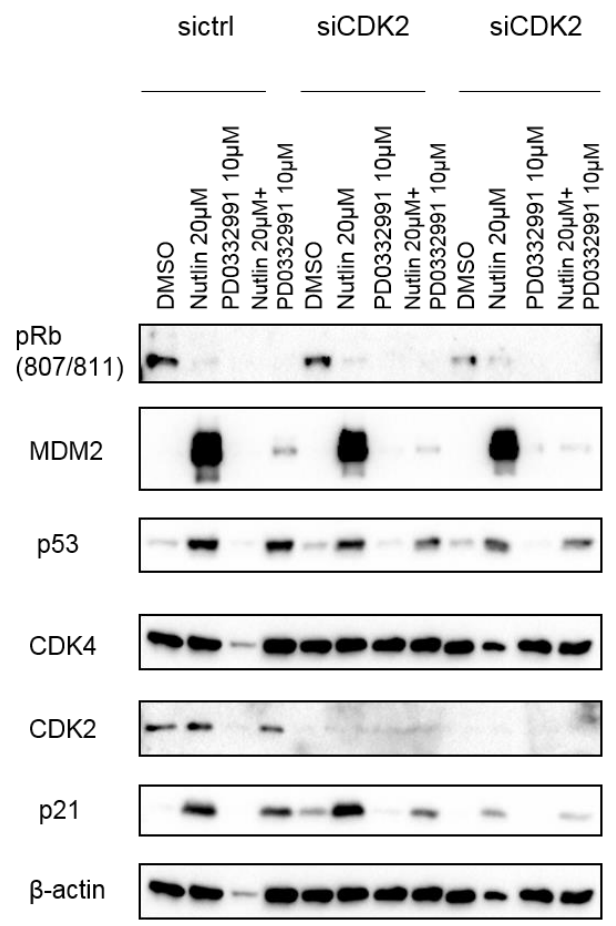

Figure 5 A 3 MDM2 stability and expression remains largely unaffected upon depletion of CDK2.

F. Scheme exhibiting the treatment protocol.

G. Exhausting the cells of CDK2 utilizing two siRNAs in combination with Nutlin and PD0332991 treatment did not rescue MDM2 levels. $\beta$-actin serves as loading control.

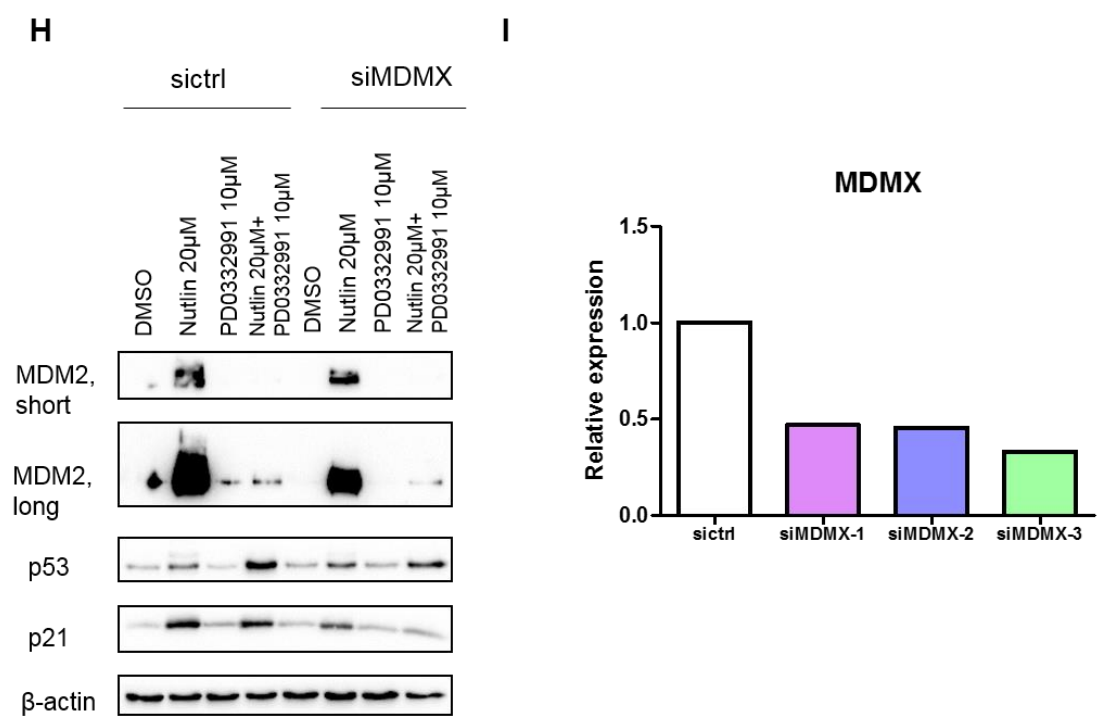

Figure 5 A 3 MDM2 stability and expression remains largely unaffected upon depletion of MDMX, its dimerization partner. 
H. Removal of MDMX from SJSA cells with Nutlin and PD0332991 treatment did not rescue the MDM2 protein levels as observed with control knockdown and the combination treatment. $\beta$ actin serves as loading control.

I. Three different siRNAs to MDMX showed decreased expression upon its depletion using quantitative RT-PCRs. The siRNA to MDMX used in $\mathrm{H}$ was a pool of the three siRNAs shown in I. RPLPO is used as housekeeping gene.

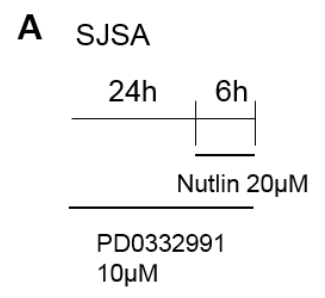

B

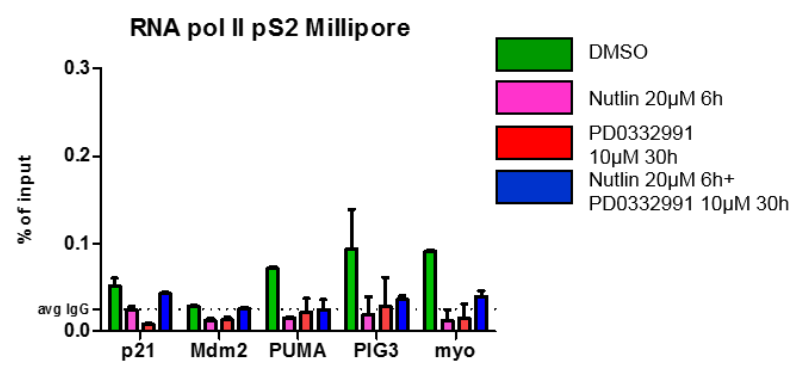

C

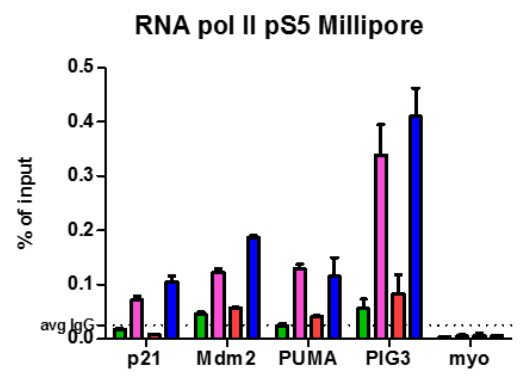

Figure 5 A 4 Combination of MDM2 antagonist with CDK4/6 inhibitor does not largely affect pS2 and pS5 RNA polymerase II at p53 target genes.

A. Protocol for treatment of SJSA cells with MDM2 antagonist and CDK4/6 inhibitor.

B. Chromatin immunoprecipitation carried out for pS2 RNA polymerase II with IgG as negative control. Targeted qPCRs were performed at the promoters of p53 target genes. The enrichment of pS2 RNA polymerase II (an indicator of transcriptional elongation) upon combined treatment of MDM2 and CDK4/6 inhibition in comparison to Nutlin alone was similar.

C. Chromatin immunoprecipitation conducted for pS5 RNA polymerase II with IgG as negative control. Targeted ChIP-qPCRs were performed at the promoters of p53 target genes. The enrichment of pS5 RNA polymerase II (represents initiation of transcription) upon combined inhibition of MDM2 and CDK4/6 in comparison to MDM2 inhibition alone was similar. 
SJSA

6h treatment

A

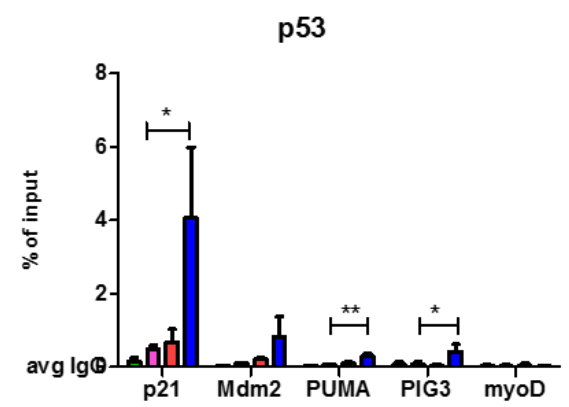

C

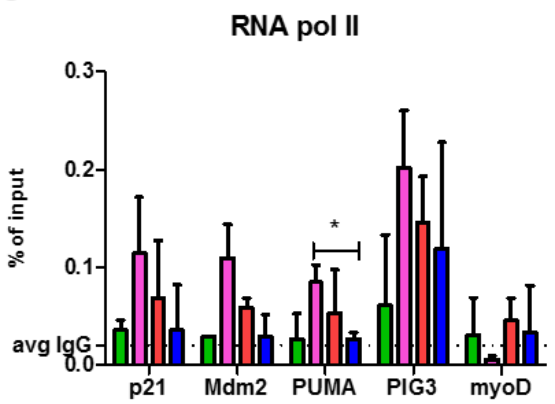

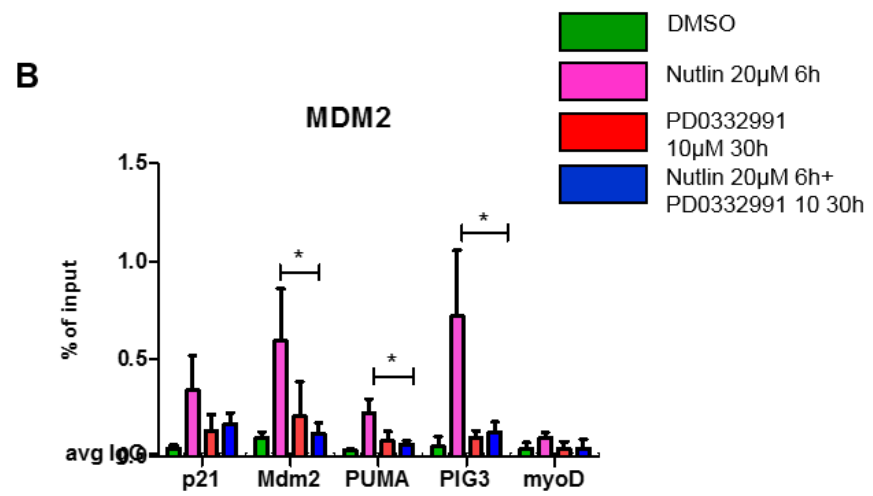

D

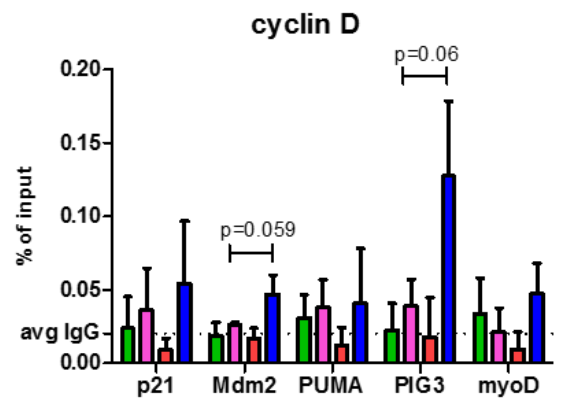

Figure 5 A 5. CDK4 and MDM2 inhibition leads to slightly elevated enrichment of cyclin D1 at the promoters of p53 target genes.

A-D. Upon performing chromatin immunoprecipitation in SJSA cells after 6 hours treatment with MDM2 and CDK4/6 inhibitors, we observed increased occupancy of p53 at its target genes upon combined MDM2 and CDK4/6 inhibition in comparison to single treatment alone. Moreover, the combined MDM2 and CDK4/6 inhibition resulted in reduced MDM2 enrichment and RNA polymerase II occupancy at these sites. Precipitation with cyclin D1 led to signals with enrichment just above the negative control IgG. In case of MDM2 and PIG3 promoters, the enrichment was increased in comparison to Nutlin treatment alone. Cyclin D1 has been suggested to act as a transcriptional regulator. In this case, it is tempting to suggest the role of cyclin D1 as a transcriptional repressor of p53 target genes. 


\section{LIST OF PUBLICATIONS}

The following publications are a part of the cumulative dissertation ,'Targeting MDM2, the antagonist of the tumor suppressor p53".

1) Anusha Sriraman, Antje Dickmanns, Zeynab Najafova, Steven Johnsen, Matthias Dobbelstein. CDK4 inhibition diminishes p53-activation by MDM2 antagonists. Manuscript under consideration at Cell Death and Disease.

2) Anusha Sriraman, Yizhu Li, Matthias Dobbelstein. Fortifying p53 - beyond Mdm2 inhibitors. Aging (Albany, NY) (2016). 2016 Sep 29. doi: 10.18632/aging.101073.

3) Anusha Sriraman, Marija Radovanovic, Magdalena Wienken, Zeynab Najafova, Yizhu $\mathrm{Li}^{*}$, and Matthias Dobbelstein*. Cooperation of Nutlin-3a with a Wip1 inhibitor to induce p53 activity. Oncotarget (2016). 10.18632/oncotarget.9302. *equal corresponding authors

4) Yizhu Li*, Priyanka Saini*, Anusha Sriraman*, Matthias Dobbelstein. Mdm2 inhibition confers protection of p53-proficient cells from the cytotoxic effects of Wee1 inhibitors. Oncotarget (2015). 10.18632/oncotarget.5891. *equal first authors 


\section{ACKNOWLEDGEMENTS}

I would like to thank my supervisor Prof. Dr. Matthias Dobbelstein for giving me this opportunity to pursue my Ph.D. in his lab. I am very grateful for his constant support, guidance, encouragement and for all the vivid and exciting scientific discussions that we have had.

I take this opportunity to thank my thesis advisory committee members Prof. Dr. Steven Johnsen and Prof. Dr. Heidi Hahn for their valuable and critical inputs and discussions.

Many thanks to Dr. Roland Dosch, PD Dr. Halyna Shcherbata, and Dr Nuno Raimundo for agreeing to be members of my extended thesis advisory committee.

I am grateful to the Göttingen Graduate School for Neurosciences, Biophysics, and Molecular Biosciences (GGNB) and the Ph.D. program Molecular Medicine for accepting me into their doctoral school.

Gratitude to Dr. Priyanka Saini, Yizhu Li and Marija Radovanovic for their contributions before I started working on these projects. I would like to thank everyone who were instrumental in bringing these stories and projects together. I express my gratitude to Dr. Zeynab Najafova and Dr. Magdalena Wienken for their help in teaching me RNA-sequencing data analysis and Antje Dickmanns for her work in carrying out co-immunoprecipitations. I learnt a lot from the supervision of my lab rotation students and I thank Nandhini Muniasamy and Dilantha Perera for their contributions towards the projects.

Heartfelt thanks to all the past and present members of the third floor of GZMB. The emotional support, laughter and all the fun times we have shared was an integral part of this journey. I would like to thank the past and present office mates - Josephine Choo, Ina Klusmann, Dr. Florian Wegwitz, Dr. Vijayalakshmi Kari, Dr. Simon Baumgart, Dr. Robyn Kosinsky, Feda Hamdan and Dr. Sankari Nagarajan for their amazing company.

I thank Bharat, Veena, Ina, Josephine, Deepa, Raghu, Ankit, Ritu, Merit and Jayaram Athimber for proofreading this thesis.

Finally, I extend my heartfelt gratitude to my family especially my mother, grandmother and brother for being my pillar of support and for their constant encouragement and belief in me. I thank them, my friends and my fiancé for hearing me out and for their support over the last years. 


\section{ABBREVIATIONS}

\begin{tabular}{|c|c|}
\hline$\%$ & percentage \\
\hline$\mu g$ & microgram \\
\hline$\mu l$ & microliter \\
\hline$\mu \mathrm{M}$ & micromolar \\
\hline $5-\mathrm{FU}$ & 5-fluorouracil \\
\hline ACC & Adrenocortical carcinoma \\
\hline AML & Acute Myeloid Leukemia \\
\hline APAF-1 & Apoptosis-peptidase activating factor- 1 \\
\hline ARF & Alternate reading frame \\
\hline ATM & Ataxia Telangiectasia Mutated \\
\hline ATR & Ataxia Telangiectasia and Rad-3 related \\
\hline bam & Binary version of SAM files \\
\hline BAX & Bcl-2-Associated $X$ protein \\
\hline $\mathrm{BCA}$ & Bicinchoninic acid \\
\hline $\mathrm{Bcl}-2$ & B-cell lymphoma 2 \\
\hline BTG2 & BTG anti-proliferation factor 2 \\
\hline Caspases & Cysteine aspartic proteases \\
\hline CBP & cAMP-response element -binding protein \\
\hline CCND1 & Cyclin D1 \\
\hline CDK & Cell-cycle dependent kinase \\
\hline ChIP & Chromatin Immunoprecipitation \\
\hline $\mathrm{Cl}$ & combination index \\
\hline CK2 & Casein Kinase 2 \\
\hline CLL & Chronic Lymphoid Leukemia \\
\hline $\mathrm{CO}_{2}$ & Carbon dioxide \\
\hline Co-IP & Co-immunoprecipitation \\
\hline COP1 & COnstitutively Photomorphogenic 1 \\
\hline CTD & C-terminal domain \\
\hline DBD & DNA binding domain \\
\hline DDB2 & DNA damage binding protein 2 \\
\hline DDR & DNA damage response \\
\hline DMEM & Dulbecco's modified Eagle's medium \\
\hline DMSO & Dimethyl sulfoxide \\
\hline DNA-PK & DNA-dependent protein kinase \\
\hline DRB & 5,6-dichloro-1- $\beta$-D-ribofuranosylbenzimidazole \\
\hline
\end{tabular}




\begin{tabular}{|c|c|}
\hline DREAM & MuvB-p107/p130-DP-E2F4 \\
\hline DSB & DNA-Double-Strand Breaks \\
\hline DUBs & Deubiquitinating enzymes \\
\hline EdU & 5-ethynyl-2'-deoxyuridine \\
\hline ER & Estrogen Receptor \\
\hline ES & Enrichment score \\
\hline Fas ligand & Fas-L \\
\hline Fbx11 & F-box protein 11 \\
\hline FDA & Food and Drug Administration \\
\hline FDR & False discovery rate \\
\hline GADD45a & Growth- arrest and DNA-damage-inducible $45 \alpha$ \\
\hline Gemcitabine & 2', 2'-difluorodeoxycytidine \\
\hline GRO-Seq & Global-Run on sequencing \\
\hline GSEA & Gene set enrichment analysis \\
\hline H3 & Histone3 \\
\hline H3K27ac & Acetylation of histone $\mathrm{H} 3$ at lysine 27 \\
\hline HATs & Histone acetyltransferases \\
\hline HAUSP & Herpes-Specific Ubiquitin Specific Protease \\
\hline HDACs & Histone deacetylases \\
\hline HER & Human Epidermal receptor \\
\hline hg19 & Human Genome project version 19 \\
\hline HIPK2 & Homeodomain interacting protein kinase 2 \\
\hline HPV-E6 & Human Papillomavirus E6 \\
\hline IC & Inhibitory concentration \\
\hline ICAD & Inhibitor of the caspase-activated DNAse \\
\hline $\lg G$ & Immunoglobulin G \\
\hline JNK & Jun $\mathrm{NH}_{2}$-terminal kinase \\
\hline $\mathrm{kb}$ & Kilo base pairs \\
\hline $\mathrm{kg}$ & Kilo gram \\
\hline M & Molar \\
\hline $\mathrm{M} / \mathrm{R} / \mathrm{N}$ & Mre11/Rad50/Nbs1 \\
\hline MDM2 & Murine double-minute 2 \\
\hline $\mathrm{mg}$ & Milli gram \\
\hline $\mathrm{MI}$ & Myocardial infarction \\
\hline MK-1775 & Wee1 inhibitor \\
\hline $\mathrm{ml}$ & Milli liter \\
\hline $\mathrm{mM}$ & Milli molar \\
\hline
\end{tabular}




$\begin{array}{ll}\text { MSigDB } & \text { Molecular Signatures Database } \\ \text { mTOR } & \text { Mammalian target of rapamycin } \\ \text { nM } & \text { Nano molar } \\ \text { n.s. } & \text { not significant } \\ \text { NCS } & \text { Neocarzinostatin } \\ \text { NER } & \text { Nucleotide excision repair } \\ \text { NES } & \text { Nuclear export signal } \\ \text { NF-kB } & \text { Nuclear factor 'kappa-light-chain-enhancer' of activated B-cells } \\ \text { Nutlin } & \text { Nutlin-3a } \\ \text { p-value } & \text { Probability value of rejectign a null hypothesis } \\ \text { PACT } & \text { p53-associated cellular protein-testes derived } \\ \text { PAI 1 } & \text { Plasminogen Activator Inhibitor-1 } \\ \text { PARP } & \text { Poly- ADP ribose polymerase } \\ \text { PcG } & \text { Polycomb group } \\ \text { PCNA } & \text { Proliferating cell nuclear antigen } \\ \text { PI } & \text { Propidium iodide } \\ \text { PML } & \text { Promyelocytic Leukemia protein } \\ \text { POLH } & \text { Pol eta } \\ \text { PP } & \text { Proline rich domain } \\ \text { PRC } & \text { Polycomb repressive complex } \\ \text { PRIMA 1 } & \text { p53-reactivation and induction of massive apoptosis 1 } \\ \text { PTM } & \text { Post-translational modifications } \\ \text { PUMA } & \text { p53 Upregulated Modulator of Apoptosis } \\ \text { qPCR } & \text { Quantitative real-time PCR } \\ \text { RAD21 } & \text { Double-strand break repair protein } \\ \text { Rb } & \text { Retinoblastoma } \\ \text { RECQL4 } & \text { ATP-dependent DNA helicase Q4 } \\ \text { RG-7388 } & \text { Idasanutlin } \\ \text { RING } & \text { Really Interesting New Gene } \\ \text { RNA pol II } & \text { RNA polymerase II } \\ \text { RNA-Seq } & \text { RNA sequencing analysis } \\ \text { RT } & \text { Room temperature } \\ \text { SAB } & \text { Senescence-associated beta-galactosidase } \\ \text { siRNA } & \text { Small interfering RNA } \\ \text { SIRT1 } & \text { Sirtuin1 } \\ \text { TAD } & \text { Transactivation domains } \\ \text { TAFII250 } & \text { TATA-box binding protein associated factor 250kDa } \\ & \end{array}$




$\begin{array}{ll}\text { TBP } & \text { TATA-box binding protein } \\ \text { TFIIH } & \text { Transcription factor II H } \\ \text { TLR } & \text { Toll-like receptor } \\ \text { TLS } & \text { Translesion synthesis } \\ \text { TNFS6 } & \text { Tumor necrosis factor S6 } \\ \text { TNF- } \alpha & \text { Tumor-necrosis factor- } \alpha \\ \text { TRAIL } & \text { Tumor necrosis factor-related apoptosis-inducing ligand } \\ \text { TRRAP } & \text { Transformation/transcription domain-associated protein } \\ \text { TSS } & \text { Transcription start site } \\ \text { UTR } & \text { Untranslated region } \\ \text { UV } & \text { Ultraviolet } \\ \text { WIP1 } & \text { Wild-type p53 induced phosphatase } \\ \text { WT } & \text { Wildtype } \\ \text { XPC2 } & \text { Xeroderma pigmentosum complementation group C } \\ \text { YH2AX } & \text { Phosphorylation of Histone2AX }\end{array}$




\section{REFERENCES}

1. Kress, M., et al., Simian virus 40-transformed cells express new species of proteins precipitable by anti-simian virus 40 tumor serum. J Virol, 1979. 31(2): p. 472-83.

2. Lane, D.P. and L.V. Crawford, T antigen is bound to a host protein in SV40-transformed cells. Nature, 1979. 278(5701): p. 261-3.

3. Linzer, D.I. and A.J. Levine, Characterization of a 54K dalton cellular SV40 tumor antigen present in SV40-transformed cells and uninfected embryonal carcinoma cells. Cell, 1979. 17(1): p. 43-52.

4. Levine, A.J., J. Momand, and C.A. Finlay, The p53 tumour suppressor gene. Nature, 1991. 351(6326): p. 453-6.

5. Hollstein, M., et al., p53 mutations in human cancers. Science, 1991. 253(5015): p. 49-53.

6. $\quad \mathrm{Li}$, F.P. and J.F. Fraumeni, Jr., Soft-tissue sarcomas, breast cancer, and other neoplasms. A familial syndrome? Ann Intern Med, 1969. 71(4): p. 747-52.

7. Li, F.P. and J.F. Fraumeni, Jr., Prospective study of a family cancer syndrome. Jama, 1982. 247(19): p. 2692-4.

8. Li, F.P., et al., A cancer family syndrome in twenty-four kindreds. Cancer Res, 1988. 48(18): $p$. 5358-62.

9. Garber, J.E., et al., Follow-up study of twenty-four families with Li-Fraumeni syndrome. Cancer Res, 1991. 51(22): p. 6094-7.

10. Donehower, L.A., et al., Mice deficient for p53 are developmentally normal but susceptible to spontaneous tumours. Nature, 1992. 356(6366): p. 215-21.

11. Kaghad, M., et al., Monoallelically expressed gene related to p53 at 1p36, a region frequently deleted in neuroblastoma and other human cancers. Cell, 1997. 90(4): p. 809-19.

12. Yang, A., et al., p63, a p53 homolog at 3q27-29, encodes multiple products with transactivating, death-inducing, and dominant-negative activities. Mol Cell, 1998. 2(3): p. 305-16.

13. Gong, J.G., et al., The tyrosine kinase $c-A b l$ regulates $p 73$ in apoptotic response to cisplatininduced DNA damage. Nature, 1999. 399(6738): p. 806-9.

14. Nemajerova, A., et al., TAp73 is a central transcriptional regulator of airway multiciliogenesis. Genes Dev, 2016. 30(11): p. 1300-12.

15. Yang, A., et al., p73-deficient mice have neurological, pheromonal and inflammatory defects but lack spontaneous tumours. Nature, 2000. 404: p. 99.

16. Talos, F., et al., p73 suppresses polyploidy and aneuploidy in the absence of functional p53. Mol Cell, 2007. 27(4): p. 647-59.

17. Mills, A.A., et al., p63 is a p53 homologue required for limb and epidermal morphogenesis. Nature, 1999. 398(6729): p. 708-13.

18. Yang, A., et al., p63 is essential for regenerative proliferation in limb, craniofacial and epithelial development. Nature, 1999. 398(6729): p. 714-8.

19. el-Deiry, W.S., et al., Definition of a consensus binding site for p53. Nat Genet, 1992. 1(1): p. 45-9.

20. Funk, W.D., et al., A transcriptionally active DNA-binding site for human $p 53$ protein complexes. Mol Cell Biol, 1992. 12(6): p. 2866-71.

21. Contente, A., et al., A polymorphic microsatellite that mediates induction of PIG3 by p53. Nat Genet, 2002. 30(3): p. 315-20.

22. Farmer, G., et al., Wild-type p53 activates transcription in vitro. Nature, 1992. 358: p. 83.

23. Shaw, P., et al., Induction of apoptosis by wild-type p53 in a human colon tumor-derived cell line. Proc Natl Acad Sci U S A, 1992. 89(10): p. 4495-9.

24. Kortlever, R.M., P.J. Higgins, and R. Bernards, Plasminogen activator inhibitor-1 is a critical downstream target of $p 53$ in the induction of replicative senescence. Nat Cell Biol, 2006. 8(8): p. 877-84.

25. Brown, J.P., W. Wei, and J.M. Sedivy, Bypass of senescence after disruption of p21CIP1/WAF1 gene in normal diploid human fibroblasts. Science, 1997. 277(5327): p. 831-4.

26. Dai, C. and W. Gu, p53 post-translational modification: deregulated in tumorigenesis. Trends Mol Med, 2010. 16(11): p. 528-36. 
27. Chang, J., et al., Transactivation ability of p53 transcriptional activation domain is directly related to the binding affinity to TATA-binding protein. J Biol Chem, 1995. 270(42): p. 25014-9.

28. Dawson, R., et al., The N-terminal domain of p53 is natively unfolded. J Mol Biol, 2003. 332(5): p. $1131-41$.

29. Ferreon, J.C., et al., Cooperative regulation of p53 by modulation of ternary complex formation with CBP/p300 and HDM2. Proc Natl Acad Sci U S A, 2009. 106(16): p. 6591-6.

30. Appella, E. and C.W. Anderson, Post-translational modifications and activation of p53 by genotoxic stresses. Eur J Biochem, 2001. 268(10): p. 2764-72.

31. Meek, D.W. and C.W. Anderson, Posttranslational modification of p53: cooperative integrators of function. Cold Spring Harb Perspect Biol, 2009. 1(6): p. a000950.

32. Léveillard, T., et al., Functional interactions between p53 and the TFIIH complex are affected by tumour-associated mutations. Embo j, 1996. 15(7): p. 1615-24.

33. Walker, K.K. and A.J. Levine, Identification of a novel p53 functional domain that is necessary for efficient growth suppression. Proc Natl Acad Sci U S A, 1996. 93(26): p. 15335-40.

34. Venot, C., et al., The requirement for the p53 proline-rich functional domain for mediation of apoptosis is correlated with specific PIG3 gene transactivation and with transcriptional repression. Embo j, 1998. 17(16): p. 4668-79.

35. Sakamuro, D., et al., The polyproline region of p53 is required to activate apoptosis but not growth arrest. Oncogene, 1997. 15(8): p. 887-98.

36. Pavletich, N.P., K.A. Chambers, and C.O. Pabo, The DNA-binding domain of p53 contains the four conserved regions and the major mutation hot spots. Genes Dev, 1993. 7(12b): p. 2556-64.

37. Kraiss, S., et al., Oligomerization of oncoprotein p53. J Virol, 1988. 62(12): p. 4737-44.

38. Stommel, J.M., et al., A leucine-rich nuclear export signal in the p53 tetramerization domain: regulation of subcellular localization and p53 activity by NES masking. Embo j, 1999. 18(6): p. 1660-72.

39. Kubbutat, M.H., et al., Regulation of Mdm2-directed degradation by the $C$ terminus of $p 53$. Mol Cell Biol, 1998. 18(10): p. 5690-8.

40. Haupt, Y., et al., Mdm2 promotes the rapid degradation of p53. Nature, 1997. 387(6630): p. 2969.

41. Honda, R., H. Tanaka, and H. Yasuda, Oncoprotein MDM2 is a ubiquitin ligase E3 for tumor suppressor p53. FEBS Lett, 1997. 420(1): p. 25-7.

42. Barak, Y., et al., mdm2 expression is induced by wild type p53 activity. Embo j, 1993. 12(2): p. 461-8.

43. $\mathrm{Li}$, L., et al., PACT is a negative regulator of $p 53$ and essential for cell growth and embryonic development. Proc Natl Acad Sci U S A, 2007. 104(19): p. 7951-6.

44. Leng, R.P., et al., Pirh2, a p53-induced ubiquitin-protein ligase, promotes p53 degradation. Cell, 2003. 112(6): p. 779-91.

45. Dornan, D., et al., The ubiquitin ligase COP1 is a critical negative regulator of p53. Nature, 2004. 429(6987): p. 86-92.

46. Castellino, R.C., et al., Medulloblastomas overexpress the p53-inactivating oncogene WIP1/PPM1D. J Neurooncol, 2008. 86(3): p. 245-56.

47. Takekawa, M., et al., p53-inducible Wip1 phosphatase mediates a negative feedback regulation of p38 MAPK-p53 signaling in response to UV radiation. Embo j, 2000. 19(23): p. 6517-26.

48. Chène, P., Inhibiting the p53-MDM2 interaction: an important target for cancer therapy. Nature Reviews Cancer, 2003. 3: p. 102.

49. Allen, M.A., et al., Global analysis of p53-regulated transcription identifies its direct targets and unexpected regulatory mechanisms. Elife, 2014. 3: p. e02200.

50. Fischer, M., Census and evaluation of p53 target genes. Oncogene, 2017. 36(28): p. 3943-56.

51. Jacobs, T., Control of the cell cycle. Dev Biol, 1992. 153(1): p. 1-15.

52. Deng, C., et al., Mice lacking p21CIP1/WAF1 undergo normal development, but are defective in G1 checkpoint control. Cell, 1995. 82(4): p. 675-84.

53. el-Deiry, W.S., et al., WAF1, a potential mediator of p53 tumor suppression. Cell, 1993. 75(4): p. 817-25.

54. Brugarolas, J., et al., Radiation-induced cell cycle arrest compromised by p21 deficiency. Nature, 1995. 377(6549): p. 552-7. 
55. Galbiati, F., et al., Caveolin-1 expression negatively regulates cell cycle progression by inducing $G(0) / G(1)$ arrest via a p53/p21(WAF1/Cip1)-dependent mechanism. Mol Biol Cell, 2001. 12(8): p. 2229-44.

56. Rouault, J.P., et al., Identification of BTG2, an antiproliferative p53-dependent component of the DNA damage cellular response pathway. Nat Genet, 1996. 14(4): p. 482-6.

57. Wang, X.W., et al., GADD45 induction of a G2/M cell cycle checkpoint. Proc Natl Acad Sci U S A, 1999. 96(7): p. 3706-11.

58. Chan, T.A., et al., 14-3-3Sigma is required to prevent mitotic catastrophe after DNA damage. Nature, 1999. 401(6753): p. 616-20.

59. Tan, T. and G. Chu, p53 Binds and activates the xeroderma pigmentosum DDB2 gene in humans but not mice. Mol Cell Biol, 2002. 22(10): p. 3247-54.

60. Adimoolam, S. and J.M. Ford, p53 and DNA damage-inducible expression of the xeroderma pigmentosum group C gene. Proc Natl Acad Sci U S A, 2002. 99(20): p. 12985-90.

61. Moldovan, G.L., B. Pfander, and S. Jentsch, PCNA, the maestro of the replication fork. Cell, 2007. 129(4): p. 665-79.

62. Shivakumar, C.V., et al., Wild-type human p53 transactivates the human proliferating cell nuclear antigen promoter. Mol Cell Biol, 1995. 15(12): p. 6785-93.

63. Essers, J., et al., Nuclear dynamics of PCNA in DNA replication and repair. Mol Cell Biol, 2005. 25(21): p. 9350-9.

64. Lerner, L.K., et al., Predominant role of DNA polymerase eta and p53-dependent translesion synthesis in the survival of ultraviolet-irradiated human cells. Nucleic Acids Res, 2017. 45(3): p. 1270-1280.

65. Liu, G. and X. Chen, DNA polymerase eta, the product of the xeroderma pigmentosum variant gene and a target of p53, modulates the DNA damage checkpoint and p53 activation. Mol Cell Biol, 2006. 26(4): p. 1398-413.

66. Schulze-Osthoff, K., et al., Apoptosis signaling by death receptors. Eur J Biochem, 1998. 254(3): p. 439-59.

67. Tsujimoto, Y., Cell death regulation by the Bcl-2 protein family in the mitochondria. J Cell Physiol, 2003. 195(2): p. 158-67.

68. Muller, M., et al., p53 activates the CD95 (APO-1/Fas) gene in response to DNA damage by anticancer drugs. J Exp Med, 1998. 188(11): p. 2033-45.

69. Munsch, D., et al., Human and mouse Fas (APO-1/CD95) death receptor genes each contain a p53-responsive element that is activated by p53 mutants unable to induce apoptosis. $\mathrm{J}$ Biol Chem, 2000. 275(6): p. 3867-72.

70. Schilling, T., et al., Active transcription of the human FAS/CD95/TNFRSF6 gene involves the p53 family. Biochem Biophys Res Commun, 2009. 387(2): p. 399-404.

71. Takimoto, R. and W.S. El-Deiry, Wild-type p53 transactivates the KILLER/DR5 gene through an intronic sequence-specific DNA-binding site. Oncogene, 2000. 19(14): p. 1735-43.

72. Liu, X., et al., p53 upregulates death receptor 4 expression through an intronic $p 53$ binding site. Cancer Res, 2004. 64(15): p. 5078-83.

73. Miyashita, T. and J.C. Reed, Tumor suppressor p53 is a direct transcriptional activator of the human bax gene. Cell, 1995. 80(2): p. 293-9.

74. Nakano, K. and K.H. Vousden, PUMA, a novel proapoptotic gene, is induced by p53. Mol Cell, 2001. 7(3): p. 683-94.

75. Shibue, T., et al., Integral role of Noxa in p53-mediated apoptotic response. Genes Dev, 2003. 17(18): p. 2233-8.

76. Fortin, A., APAF1 is a key transcriptional target for $p 53$ in the regulation of neuronal cell death. 2001. 155(2): p. 207-16.

77. Robles, A.I., et al., APAF-1 is a transcriptional target of p53 in DNA damage-induced apoptosis. Cancer Res, 2001. 61(18): p. 6660-4.

78. Pearson, M., et al., PML regulates p53 acetylation and premature senescence induced by oncogenic Ras. Nature, 2000. 406(6792): p. 207-10.

79. Aksoy, O., et al., The atypical E2F family member E2F7 couples the p53 and RB pathways during cellular senescence. Genes Dev, 2012. 26(14): p. 1546-57.

80. Brookes, S., et al., INK4a-deficient human diploid fibroblasts are resistant to RAS-induced senescence. 2002. 21(12): p. 2936-45. 
81. Roninson, I.B., Oncogenic functions of tumour suppressor p21(Waf1/Cip1/Sdi1): association with cell senescence and tumour-promoting activities of stromal fibroblasts. Cancer Lett, 2002. 179(1): p. 1-14.

82. Lin, A.W., et al., Premature senescence involving p53 and p16 is activated in response to constitutive MEK/MAPK mitogenic signaling. Genes Dev, 1998. 12(19): p. 3008-19.

83. Sun, P., et al., PRAK is essential for ras-induced senescence and tumor suppression. Cell, 2007. 128(2): p. 295-308.

84. Xu, Y., et al., Emerging roles of the p38 MAPK and PI3K/AKT/mTOR pathways in oncogeneinduced senescence. Trends Biochem Sci, 2014. 39(6): p. 268-76.

85. Khanna, K.K., et al., ATM associates with and phosphorylates p53: mapping the region of interaction. Nature Genetics, 1998. 20: p. 398.

86. Canman, C.E., et al., Activation of the ATM kinase by ionizing radiation and phosphorylation of p53. Science, 1998. 281(5383): p. 1677-9.

87. Banin, S., et al., Enhanced phosphorylation of p53 by ATM in response to DNA damage. Science, 1998. 281(5383): p. 1674-7.

88. Tibbetts, R.S., et al., A role for ATR in the DNA damage-induced phosphorylation of $p 53$. Genes Dev, 1999. 13(2): p. 152-7.

89. Lees-Miller, S.P., et al., Human DNA-activated protein kinase phosphorylates serines 15 and 37 in the amino-terminal transactivation domain of human p53. Mol Cell Biol, 1992. 12(11): p. 50419.

90. Shieh, S.Y., et al., DNA damage-induced phosphorylation of p53 alleviates inhibition by MDM2. Cell, 1997. 91(3): p. 325-34.

91. Shieh, S.Y., et al., The human homologs of checkpoint kinases Chk1 and Cds1 (Chk2) phosphorylate p53 at multiple DNA damage-inducible sites. Genes Dev, 2000. 14(3): p. 289300.

92. Milne, D.M., et al., p53 is phosphorylated in vitro and in vivo by an ultraviolet radiation-induced protein kinase characteristic of the c-Jun kinase, JNK1. J Biol Chem, 1995. 270(10): p. 5511-8.

93. Adler, V., et al., Conformation-dependent phosphorylation of p53. Proc Natl Acad Sci U S A, 1997. 94(5): p. 1686-91.

94. Hu, M.C., W.R. Qiu, and Y.P. Wang, JNK1, JNK2 and JNK3 are p53 N-terminal serine 34 kinases. Oncogene, 1997. 15(19): p. 2277-87.

95. Bulavin, D.V., et al., Phosphorylation of human p53 by p38 kinase coordinates $N$-terminal phosphorylation and apoptosis in response to UV radiation. Embo j, 1999. 18(23): p. 6845-54.

96. Saito, S., et al., ATM mediates phosphorylation at multiple p53 sites, including Ser(46), in response to ionizing radiation. J Biol Chem, 2002. 277(15): p. 12491-4.

97. Sakaguchi, K., et al., Damage-mediated phosphorylation of human p53 threonine 18 through a cascade mediated by a casein 1-like kinase. Effect on Mdm2 binding. J Biol Chem, 2000. 275(13): p. 9278-83.

98. Buschmann, T., et al., Jun NH(2)-Terminal Kinase Phosphorylation of p53 on Thr-81 Is Important for p53 Stabilization and Transcriptional Activities in Response to Stress. Mol Cell Biol, 2001. 21(8): p. 2743-54.

99. Huang, C., et al., p38 kinase mediates UV-induced phosphorylation of p53 protein at serine 389. J Biol Chem, 1999. 274(18): p. 12229-35.

100. D'Orazi, G., et al., Homeodomain-interacting protein kinase-2 phosphorylates $p 53$ at Ser 46 and mediates apoptosis. Nat Cell Biol, 2002. 4(1): p. 11-9.

101. Hofmann, T.G., et al., Regulation of $p 53$ activity by its interaction with homeodomain-interacting protein kinase-2. Nat Cell Biol, 2002. 4(1): p. 1-10.

102. Gu, W. and R.G. Roeder, Activation of $p 53$ sequence-specific DNA binding by acetylation of the p53 C-terminal domain. Cell, 1997. 90(4): p. 595-606.

103. Sykes, S.M., et al., Acetylation of the p53 DNA-binding domain regulates apoptosis induction. Mol Cell, 2006. 24(6): p. 841-51.

104. Tang, Y., et al., Tip60-dependent acetylation of p53 modulates the decision between cell-cycle arrest and apoptosis. Mol Cell, 2006. 24(6): p. 827-39.

105. Abida, W.M., et al., FBXO11 promotes the Neddylation of p53 and inhibits its transcriptional activity. J Biol Chem, 2007. 282(3): p. 1797-804. 
106. Xirodimas, D.P., et al., Mdm2-mediated NEDD8 conjugation of p53 inhibits its transcriptional activity. Cell, 2004. 118(1): p. 83-97.

107. Rodriguez, M.S., et al., SUMO-1 modification activates the transcriptional response of p53. Embo j, 1999. 18(22): p. 6455-61.

108. Fiscella, M., et al., Wip1, a novel human protein phosphatase that is induced in response to ionizing radiation in a p53-dependent manner. Proc Natl Acad Sci U S A, 1997. 94(12): p. 604853.

109. Ofek, P., et al., Cell cycle regulation and $p 53$ activation by protein phosphatase $2 \mathrm{C}$ alpha. J Biol Chem, 2003. 278(16): p. 14299-305.

110. Vaziri, H., et al., hSIR2(SIRT1) functions as an NAD-dependent p53 deacetylase. Cell, 2001. 107(2): p. 149-59.

111. Luo, J., et al., Negative control of p53 by Sir2alpha promotes cell survival under stress. Cell, 2001. 107(2): p. 137-48.

112. Chuikov, S., et al., Regulation of p53 activity through lysine methylation. Nature, 2004. 432(7015): p. 353-60.

113. Rodriguez, M.S., et al., Multiple C-Terminal Lysine Residues Target p53 for UbiquitinProteasome-Mediated Degradation. Mol Cell Biol, 2000. 20(22): p. 8458-67.

114. Lohrum, M.A., et al., C-terminal ubiquitination of p53 contributes to nuclear export. Mol Cell Biol, 2001. 21(24): p. 8521-32.

115. Li, M., et al., Mono- versus polyubiquitination: differential control of p53 fate by Mdm2. Science, 2003. 302(5652): p. 1972-5.

116. Chen, D., et al., ARF-BP1/Mule is a critical mediator of the ARF tumor suppressor. Cell, 2005. 121(7): p. 1071-83.

117. Li, M., et al., Deubiquitination of p53 by HAUSP is an important pathway for p53 stabilization. Nature, 2002. 416(6881): p. 648-53.

118. Cahilly-Snyder, L., et al., Molecular analysis and chromosomal mapping of amplified genes isolated from a transformed mouse 3 T3 cell line. Somat Cell Mol Genet, 1987. 13(3): p. 235-44.

119. Momand, J., et al., The $m d m-2$ oncogene product forms a complex with the p53 protein and inhibits p53-mediated transactivation. Cell, 1992. 69(7): p. 1237-45.

120. Oliner, J.D., et al., Amplification of a gene encoding a p53-associated protein in human sarcomas. Nature, 1992. 358(6381): p. 80-3.

121. Jones, S.N., et al., Rescue of embryonic lethality in Mdm2-deficient mice by absence of p53. Nature, 1995. 378(6553): p. 206-8.

122. Montes de Oca Luna, R., D.S. Wagner, and G. Lozano, Rescue of early embryonic lethality in mdm2-deficient mice by deletion of p53. Nature, 1995. 378(6553): p. 203-6.

123. Watanabe, T., et al., Overexpression of the MDM2 oncogene in leukemia and lymphoma. Leuk Lymphoma, 1996. 21(5-6): p. 391-7, color plates XVI following 5.

124. Kussie, P.H., et al., Structure of the MDM2 oncoprotein bound to the p53 tumor suppressor transactivation domain. Science, 1996. 274(5289): p. 948-53.

125. Wade, M., Y.V. Wang, and G.M. Wahl, The p53 orchestra: Mdm2 and Mdmx set the tone. Trends Cell Biol, 2010. 20(5): p. 299-309.

126. Sdek, P., et al., The central acidic domain of MDM2 is critical in inhibition of retinoblastomamediated suppression of E2F and cell growth. J Biol Chem, 2004. 279(51): p. 53317-22.

127. Pomerantz, J., et al., The Ink4a tumor suppressor gene product, p19Arf, interacts with MDM2 and neutralizes MDM2's inhibition of p53. Cell, 1998. 92(6): p. 713-23.

128. Zhang, Y., Y. Xiong, and W.G. Yarbrough, ARF promotes MDM2 degradation and stabilizes p53: ARF-INK4a locus deletion impairs both the Rb and p53 tumor suppression pathways. Cell, 1998. 92(6): p. 725-34.

129. Weber, J.D., et al., Nucleolar Arf sequesters Mdm2 and activates p53. Nat Cell Biol, 1999. 1(1): p. 20-6.

130. Grossman, S.R., et al., p300/MDM2 complexes participate in MDM2-mediated p53 degradation. Mol Cell, 1998. 2(4): p. 405-15.

131. Zhang, Y., et al., Ribosomal protein $L 11$ negatively regulates oncoprotein MDM2 and mediates a p53-dependent ribosomal-stress checkpoint pathway. Mol Cell Biol, 2003. 23(23): p. 8902-12. 
132. Lindstrom, M.S., et al., Cancer-associated mutations in the MDM2 zinc finger domain disrupt ribosomal protein interaction and attenuate MDM2-induced p53 degradation. Mol Cell Biol, 2007. 27(3): p. 1056-68.

133. Chen, J., V. Marechal, and A.J. Levine, Mapping of the $p 53$ and mdm-2 interaction domains. Mol Cell Biol, 1993. 13(7): p. 4107-14.

134. Bottger, A., et al., Molecular characterization of the hdm2-p53 interaction. J Mol Biol, 1997. 269(5): p. 744-56.

135. Freedman, D.A., et al., A genetic approach to mapping the p53 binding site in the MDM2 protein. Mol Med, 1997. 3(4): p. 248-59.

136. Schon, O., et al., Molecular mechanism of the interaction between MDM2 and p53. J Mol Biol, 2002. 323(3): p. 491-501.

137. Honda, $\mathrm{R}$. and $\mathrm{H}$. Yasuda, Activity of MDM2, a ubiquitin ligase, toward $p 53$ or itself is dependent on the RING finger domain of the ligase. Oncogene, 2000. 19(11): p. 1473-6.

138. Fang, S., et al., Mdm2 is a RING finger-dependent ubiquitin protein ligase for itself and $p 53 . \mathrm{J}$ Biol Chem, 2000. 275(12): p. 8945-51.

139. Lai, Z., et al., Human mdm2 mediates multiple mono-ubiquitination of p53 by a mechanism requiring enzyme isomerization. J Biol Chem, 2001. 276(33): p. 31357-67.

140. Grossman, S.R., et al., Polyubiquitination of p53 by a ubiquitin ligase activity of p300. Science, 2003. 300(5617): p. 342-4.

141. Itahana, K., et al., Targeted inactivation of Mdm2 RING finger E3 ubiquitin ligase activity in the mouse reveals mechanistic insights into p53 regulation. Cancer Cell, 2007. 12(4): p. 355-66.

142. Wang, X., et al., Inhibition of p53 degradation by Mdm2 acetylation. FEBS Lett, 2004. 561(1-3): p. 195-201.

143. Roth, J., et al., Nucleo-cytoplasmic shuttling of the hdm2 oncoprotein regulates the levels of the p53 protein via a pathway used by the human immunodeficiency virus rev protein. Embo j, 1998. 17(2): p. 554-64.

144. Tanimura, S., et al., MDM2 interacts with MDMX through their RING finger domains. FEBS Lett, 1999. 447(1): p. 5-9.

145. Sharp, D.A., et al., Stabilization of the MDM2 oncoprotein by interaction with the structurally related MDMX protein. J Biol Chem, 1999. 274(53): p. 38189-96.

146. Shvarts, A., et al., MDMX: a novel p53-binding protein with some functional properties of MDM2. Embo j, 1996. 15(19): p. 5349-57.

147. Francoz, S., et al., Mdm4 and Mdm2 cooperate to inhibit $p 53$ activity in proliferating and quiescent cells in vivo. Proc Natl Acad Sci U S A, 2006. 103(9): p. 3232-7.

148. Barak, Y., et al., Regulation of $\mathrm{mdm2}$ expression by $\mathrm{p53}$ : alternative promoters produce transcripts with nonidentical translation potential. Genes Dev, 1994. 8(15): p. 1739-49.

149. Zauberman, A., et al., A functional p53-responsive intronic promoter is contained within the human mdm2 gene. Nucleic Acids Res, 1995. 23(14): p. 2584-92.

150. Cheng, T.H. and S.N. Cohen, Human MDM2 isoforms translated differentially on constitutive versus p53-regulated transcripts have distinct functions in the p53/MDM2 and TSG101/MDM2 feedback control loops. Mol Cell Biol, 2007. 27(1): p. 111-9.

151. Brown, C.Y., et al., Role of two upstream open reading frames in the translational control of oncogene mdm2. Oncogene, 1999. 18: p. 5631.

152. Wade, M., Y.C. Li, and G.M. Wahl, MDM2, MDMX and p53 in oncogenesis and cancer therapy. Nat Rev Cancer, 2013. 13(2): p. 83-96.

153. Maya, R., et al., ATM-dependent phosphorylation of Mdm2 on serine 395: role in p53 activation by DNA damage. Genes Dev, 2001. 15(9): p. 1067-77.

154. Lu, X., et al., The Wip1 Phosphatase acts as a gatekeeper in the p53-Mdm2 autoregulatory loop. Cancer Cell, 2007. 12(4): p. 342-54.

155. Mayo, L.D. and D.B. Donner, A phosphatidylinositol 3-kinase/Akt pathway promotes. Proc Natl Acad Sci U S A, 2001. 98(20): p. 11598-603.

156. Mayo, L.D., J.J. Turchi, and S.J. Berberich, Mdm-2 phosphorylation by DNA-dependent protein kinase prevents interaction with p53. Cancer Res, 1997. 57(22): p. 5013-6.

157. Lai, K.P., et al., S6K1 is a multifaceted regulator of Mdm2 that connects nutrient status and DNA damage response. Embo j, 2010. 29(17): p. 2994-3006. 
158. Zhang, $\mathrm{T}$. and C. Prives, Cyclin a-CDK phosphorylation regulates MDM2 protein interactions. J Biol Chem, 2001. 276(32): p. 29702-10.

159. Goldberg, Z., et al., Tyrosine phosphorylation of Mdm2 by c-Abl: implications for p53 regulation. 2002. 21(14): p. 3715-27.

160. Xirodimas, D.P., et al., P14ARF promotes accumulation of SUMO-1 conjugated (H)Mdm2. FEBS Lett, 2002. 528(1-3): p. 207-11.

161. Bouska, A. and C.M. Eischen, Mdm2 affects genome stability independent of p53. Cancer Res, 2009. 69(5): p. 1697-701.

162. Mulay, S.R., et al., MDM2 (murine double minute-2) links inflammation and tubular cell healing during acute kidney injury in mice. Kidney Int, 2012. 81(12): p. 1199-211.

163. Riscal, R., et al., Chromatin-Bound MDM2 Regulates Serine Metabolism and Redox Homeostasis Independently of p53. Mol Cell, 2016. 62(6): p. 890-902.

164. Wienken, M., et al., MDM2 Associates with Polycomb Repressor Complex 2 and Enhances Stemness-Promoting Chromatin Modifications Independent of p53. Mol Cell, 2016. 61(1): p. 6883.

165. Wang, S., et al., Targeting the MDM2-p53 Protein-Protein Interaction for New Cancer Therapy: Progress and Challenges. Cold Spring Harb Perspect Med, 2017. 7(5).

166. Vassilev, L.T., et al., In vivo activation of the p53 pathway by small-molecule antagonists of MDM2. Science, 2004. 303(5659): p. 844-8.

167. Ray-Coquard, I., et al., Effect of the MDM2 antagonist RG7112 on the P53 pathway in patients with MDM2-amplified, well-differentiated or dedifferentiated liposarcoma: an exploratory proofof-mechanism study. Lancet Oncol, 2012. 13(11): p. 1133-40.

168. Siu, L.L., et al., Phase 1 dose escalation, food effect, and biomarker study of RG7388, a more potent second-generation MDM2 antagonist, in patients (pts) with solid tumors. Journal of Clinical Oncology, 2014. 32(15_suppl): p. 2535-2535.

169. Herman, A.G., et al., Discovery of Mdm2-MdmX E3 Ligase Inhibitors Using a Cell-Based Ubiquitination Assay. Cancer Discov, 2011. 1(4): p. 312-25.

170. Yang, Y., et al., Small molecule inhibitors of HDM2 ubiquitin ligase activity stabilize and activate p53 in cells. Cancer Cell, 2005. 7(6): p. 547-59.

171. Graves, B., et al., Activation of the p53 pathway by small-molecule-induced MDM2 and MDMX dimerization. Proc Natl Acad Sci U S A, 2012. 109(29): p. 11788-93.

172. Chang, Y.S., et al., Stapled alpha-helical peptide drug development: a potent dual inhibitor of MDM2 and MDMX for p53-dependent cancer therapy. Proc Natl Acad Sci U S A, 2013. 110(36): p. E3445-54.

173. Lehmann, S., et al., Targeting p53 in vivo: a first-in-human study with p53-targeting compound APR-246 in refractory hematologic malignancies and prostate cancer. J Clin Oncol, 2012. 30(29): p. 3633-9.

174. Bykov, V.J., et al., PRIMA-1(MET) synergizes with cisplatin to induce tumor cell apoptosis. Oncogene, 2005. 24(21): p. 3484-91.

175. Nahi, H., et al., Effects of PRIMA-1 on chronic lymphocytic leukaemia cells with and without hemizygous p53 deletion. Br J Haematol, 2004. 127(3): p. 285-91.

176. Li, Y., et al., Mdm2 inhibition confers protection of p53-proficient cells from the cytotoxic effects of Wee1 inhibitors. Oncotarget, 2015. 6(32): p. 32339-52.

177. Sriraman, A., et al., Cooperation of Nutlin-3a and a Wip1 inhibitor to induce p53 activity. Oncotarget, 2016. 7(22): p. 31623-38.

178. Pan, W., et al., Defining the minimal portion of the retinoblastoma protein that serves as an efficient substrate for cdk4 kinase/cyclin D1 complex. Carcinogenesis, 1998. 19(5): p. 765-9.

179. Thalmeier, K., et al., Nuclear factor E2F mediates basic transcription and trans-activation by E1a of the human MYC promoter. Genes Dev, 1989. 3(4): p. 527-36.

180. Blake, M.C. and J.C. Azizkhan, Transcription factor E2F is required for efficient expression of the hamster dihydrofolate reductase gene in vitro and in vivo. Mol Cell Biol, 1989. 9(11): p. 49945002.

181. O'Leary, B., R.S. Finn, and N.C. Turner, Treating cancer with selective CDK4/6 inhibitors. Nat Rev Clin Oncol, 2016. 13(7): p. 417-30.

182. Khoo, K.H., C.S. Verma, and D.P. Lane, Drugging the $p 53$ pathway: understanding the route to clinical efficacy. Nat Rev Drug Discov, 2014. 13(3): p. 217-36. 
183. Nguyen, D., et al., Reviving the guardian of the genome: Small molecule activators of p53. Pharmacol Ther, 2017. 178: p. 92-108.

184. Muller, C.R., et al., Potential for treatment of liposarcomas with the MDM2 antagonist Nutlin-3A. Int J Cancer, 2007. 121(1): p. 199-205.

185. Verreault, M., et al., Preclinical Efficacy of the MDM2 Inhibitor RG7112 in MDM2-Amplified and TP53 Wild-type Glioblastomas. Clin Cancer Res, 2016. 22(5): p. 1185-96.

186. Zhao, Y., et al., Small-molecule inhibitors of the MDM2-p53 protein-protein interaction (MDM2 Inhibitors) in clinical trials for cancer treatment. J Med Chem, 2015. 58(3): p. 1038-52.

187. Pilotti, S., et al., Molecular abnormalities in liposarcoma: role of MDM2 and CDK4-containing amplicons at 12q13-22. J Pathol, 1998. 185(2): p. 188-90.

188. Bill, K.L., et al., SAR405838: A Novel and Potent Inhibitor of the MDM2:p53 Axis for the Treatment of Dedifferentiated Liposarcoma. Clin Cancer Res, 2016. 22(5): p. 1150-60.

189. Ohnstad, H.O., et al., Correlation of TP53 and MDM2 genotypes with response to therapy in sarcoma. Cancer, 2013. 119(5): p. 1013-22.

190. Burgess, A., et al., Clinical Overview of MDM2/X-Targeted Therapies. Front Oncol, 2016. 6: p. 7.

191. Muthusamy, V., et al., Amplification of CDK4 and MDM2 in malignant melanoma. Genes Chromosomes Cancer, 2006. 45(5): p. 447-54.

192. Wunder, J.S., et al., Co-amplification and overexpression of CDK4, SAS and MDM2 occurs frequently in human parosteal osteosarcomas. Oncogene, 1999. 18(3): p. 783-8.

193. Berner, J.M., et al., Separate amplified regions encompassing CDK4 and MDM2 in human sarcomas. Genes Chromosomes Cancer, 1996. 17(4): p. 254-9.

194. Laroche-Clary, A., et al., Combined targeting of MDM2 and CDK4 is synergistic in dedifferentiated liposarcomas. J Hematol Oncol, 2017. 10(1): p. 123.

195. Kato, J., et al., Direct binding of cyclin $D$ to the retinoblastoma gene product $(p R b)$ and $p R b$ phosphorylation by the cyclin D-dependent kinase CDK4. Genes Dev, 1993. 7(3): p. 331-42.

196. Tovar, C., et al., Small-molecule MDM2 antagonists reveal aberrant p53 signaling in cancer: implications for therapy. Proc Natl Acad Sci U S A, 2006. 103(6): p. 1888-93.

197. Du, W., et al., Nutlin-3 affects expression and function of retinoblastoma protein: role of retinoblastoma protein in cellular response to nutlin-3. J Biol Chem, 2009. 284(39): p. 26315-21.

198. Italiano, A., et al., Variability of origin for the neocentromeric sequences in analphoid supernumerary marker chromosomes of well-differentiated liposarcomas. Cancer Lett, 2009. 273(2): p. 323-30.

199. Persson, F., et al., Characterization of the $12 q$ amplicons by high-resolution, oligonucleotide array CGH and expression analyses of a novel liposarcoma cell line. Cancer Lett, 2008. 260(12): p. 37-47.

200. Bieging, K.T., S.S. Mello, and L.D. Attardi, Unravelling mechanisms of p53-mediated tumour suppression. Nat Rev Cancer, 2014. 14(5): p. 359-70.

201. D'Andrea, A.D. and W.A. Haseltine, Sequence specific cleavage of DNA by the antitumor antibiotics neocarzinostatin and bleomycin. Proc Natl Acad Sci U S A, 1978. 75(8): p. 3608-12.

202. Liberzon, A., et al., The Molecular Signatures Database (MSigDB) hallmark gene set collection. Cell Syst, 2015. 1(6): p. 417-425.

203. Hallett, S.T., et al., Differential Regulation of G1 CDK Complexes by the Hsp90-Cdc37 Chaperone System. Cell Rep, 2017. 21(5): p. 1386-1398.

204. Barlev, N.A., et al., Acetylation of p53 activates transcription through recruitment of coactivators/histone acetyltransferases. Mol Cell, 2001. 8(6): p. 1243-54.

205. Sakaguchi, K., et al., DNA damage activates p53 through a phosphorylation-acetylation cascade. Genes Dev, 1998. 12(18): p. 2831-41.

206. Gerarduzzi, C., et al., Human epidermal growth factor receptor 4 (Her4) Suppresses p53 Protein via Targeting the MDMX-MDM2 Protein Complex: IMPLICATION OF A NOVEL MDMX SER314 PHOSPHOSITE. J Biol Chem, 2016. 291(50): p. 25937-25949.

207. Yang, P., et al., Downregulation of cyclin D1 sensitizes cancer cells to MDM2 antagonist Nutlin3. Oncotarget, 2016. 7(22): p. 32652-63.

208. Wang, Y. and C. Prives, Increased and altered DNA binding of human p53 by $S$ and G2/M but not G1 cyclin-dependent kinases. Nature, 1995. 376(6535): p. 88-91.

209. Pestell, R.G., New roles of cyclin D1. Am J Pathol, 2013. 183(1): p. 3-9. 
210. Bienvenu, F., et al., Transcriptional role of cyclin D1 in development revealed by a geneticproteomic screen. Nature, 2010. 463(7279): p. 374-8.

211. Tigan, A.S., et al., CDK6-a review of the past and a glimpse into the future: from cell-cycle control to transcriptional regulation. Oncogene, 2016. 35(24): p. 3083-91.

212. Casimiro, M.C., et al., ChIP sequencing of cyclin D1 reveals a transcriptional role in chromosomal instability in mice. J Clin Invest, 2012. 122(3): p. 833-43.

213. Bunz, F., et al., Disruption of p53 in human cancer cells alters the responses to therapeutic agents. J Clin Invest, 1999. 104(3): p. 263-9.

214. Sriraman, A., Y. Li, and M. Dobbelstein, Fortifying p53 - beyond Mdm2 inhibitors. Aging (Albany NY), 2016. 8(9): p. 1836-1837.

215. Obrador-Hevia, A., et al., RG7112, a small-molecule inhibitor of MDM2, enhances trabectedin response in soft tissue sarcomas. Cancer Invest, 2015. 33(9): p. 440-50.

216. Saiki, A.Y., et al., MDM2 antagonists synergize broadly and robustly with compounds targeting fundamental oncogenic signaling pathways. Oncotarget, 2014. 5(8): p. 2030-43.

217. Wang, H.Q., et al., Combined ALK and MDM2 inhibition increases antitumor activity and overcomes resistance in human ALK mutant neuroblastoma cell lines and xenograft models. Elife, 2017.6.

218. Franco, J., A.K. Witkiewicz, and E.S. Knudsen, CDK4/6 inhibitors have potent activity in combination with pathway selective therapeutic agents in models of pancreatic cancer. Oncotarget, 2014. 5(15): p. 6512-25.

219. Laroche, A., et al., MDM2 antagonists synergize with PI3K/mTOR inhibition in welldifferentiated/dedifferentiated liposarcomas. Oncotarget, 2017. 8(33): p. 53968-53977.

220. Latella, L., et al., Reconstitution of cyclin D1-associated kinase activity drives terminally differentiated cells into the cell cycle. Mol Cell Biol, 2001. 21(16): p. 5631-43.

221. Oliner, J.D., et al., Oncoprotein MDM2 conceals the activation domain of tumour suppressor p53. Nature, 1993. 362(6423): p. 857-60.

222. Denissov, S., et al., Identification of novel functional TBP-binding sites and general factor repertoires. EMBO J, 2007. 26(4): p. 944-54.

223. Levine, A.J., p53, the cellular gatekeeper for growth and division. Cell, 1997. 88(3): p. 323-31.

224. Lane, D.P., Cancer. p53, guardian of the genome. Nature, 1992. 358(6381): p. 15-6.

225. Nicholson, S.A., et al., Alterations of p14ARF, p53, and p73 genes involved in the E2F-1mediated apoptotic pathways in non-small cell lung carcinoma. Cancer Res, 2001. 61(14): p. 5636-43.

226. Lechner, M.S. and L.A. Laimins, Inhibition of p53 DNA binding by human papillomavirus E6 proteins. J Virol, 1994. 68(7): p. 4262-73.

227. Lew, D.J. and S. Kornbluth, Regulatory roles of cyclin dependent kinase phosphorylation in cell cycle control. Curr Opin Cell Biol, 1996. 8(6): p. 795-804.

228. Matheson, C.J., D.S. Backos, and P. Reigan, Targeting WEE1 Kinase in Cancer. Trends in Pharmacological Sciences, 2016. 37(10): p. 872-881.

229. Stathis, A. and A. Oza, Targeting Wee1-like protein kinase to treat cancer. Drug News Perspect, 2010. 23(7): p. 425-9.

230. Karnitz, L.M., et al., Gemcitabine-induced activation of checkpoint signaling pathways that affect tumor cell survival. Mol Pharmacol, 2005. 68(6): p. 1636-44.

231. Kranz, D. and M. Dobbelstein, Nongenotoxic p53 activation protects cells against S-phasespecific chemotherapy. Cancer Res, 2006. 66(21): p. 10274-80.

232. Blagosklonny, M.V., et al., Pretreatment with DNA-damaging agents permits selective killing of checkpoint-deficient cells by microtubule-active drugs. J Clin Invest, 2000. 105(4): p. 533-9.

233. Tokalov, S.V. and N.D. Abolmaali, Protection of $p 53$ wild type cells from taxol by nutlin-3 in the combined lung cancer treatment. BMC Cancer, 2010. 10: p. 57.

234. Carvajal, D., et al., Activation of p53 by MDM2 antagonists can protect proliferating cells from mitotic inhibitors. Cancer Res, 2005. 65(5): p. 1918-24.

235. Blagosklonny, M.V. and Z. Darzynkiewicz, Cyclotherapy: protection of normal cells and unshielding of cancer cells. Cell Cycle, 2002. 1(6): p. 375-82.

236. Sur, S., et al., A panel of isogenic human cancer cells suggests a therapeutic approach for cancers with inactivated p53. Proc Natl Acad Sci U S A, 2009. 106(10): p. 3964-9. 
237. Esfandiari, A., et al., Chemical Inhibition of Wild-Type p53-Induced Phosphatase 1 (WIP1/PPM1D) by GSK2830371 Potentiates the Sensitivity to MDM2 Inhibitors in a p53Dependent Manner. Mol Cancer Ther, 2016. 15(3): p. 379-91.

238. Pechackova, S., et al., Inhibition of WIP1 phosphatase sensitizes breast cancer cells to genotoxic stress and to MDM2 antagonist nutlin-3. Oncotarget, 2016. 7(12): p. 14458-75.

239. Binh, M.B., et al., MDM2 and CDK4 immunostainings are useful adjuncts in diagnosing welldifferentiated and dedifferentiated liposarcoma subtypes: a comparative analysis of 559 soft tissue neoplasms with genetic data. Am J Surg Pathol, 2005. 29(10): p. 1340-7.

240. Price, B.D., L. Hughes-Davies, and S.J. Park, Cdk2 kinase phosphorylates serine 315 of human p53 in vitro. Oncogene, 1995. 11(1): p. 73-80.

241. Rader, J., et al., Dual CDK4/CDK6 inhibition induces cell-cycle arrest and senescence in neuroblastoma. Clin Cancer Res, 2013. 19(22): p. 6173-82.

242. Tate, S.C., et al., Semi-mechanistic pharmacokinetic/pharmacodynamic modeling of the antitumor activity of LY2835219, a new cyclin-dependent kinase 4/6 inhibitor, in mice bearing human tumor xenografts. Clin Cancer Res, 2014. 20(14): p. 3763-74.

243. Rivadeneira, D.B., et al., Proliferative suppression by CDK4/6 inhibition: complex function of the retinoblastoma pathway in liver tissue and hepatoma cells. Gastroenterology, 2010. 138(5): p. 1920-30.

244. Kovatcheva, M., et al., MDM2 turnover and expression of ATRX determine the choice between quiescence and senescence in response to CDK4 inhibition. Oncotarget, 2015. 6(10): p. 822643.

245. Rinaldo, C., et al., MDM2-regulated degradation of HIPK2 prevents p53Ser46 phosphorylation and DNA damage-induced apoptosis. Mol Cell, 2007. 25(5): p. 739-50.

246. Zeng, X., et al., MDM2 Suppresses p73 Function without Promoting p73 Degradation. Mol Cell Biol, 1999. 19(5): p. 3257-66.

247. Gu, J., et al., Mutual dependence of MDM2 and MDMX in their functional inactivation of p53. J Biol Chem, 2002. 277(22): p. 19251-4.

248. Lu, W., et al., Activation of $p 53$ by roscovitine-mediated suppression of MDM2 expression. Oncogene, 2001. 20(25): p. 3206-16.

249. Cheok, C.F., A. Dey, and D.P. Lane, Cyclin-dependent kinase inhibitors sensitize tumor cells to nutlin-induced apoptosis: a potent drug combination. Mol Cancer Res, 2007. 5(11): p. 1133-45.

250. Olson, C.M., et al., Pharmacological perturbation of CDK9 using selective CDK9 inhibition or degradation. Nat Chem Biol, 2018. 14(2): p. 163-170.

251. Phatnani, H.P. and A.L. Greenleaf, Phosphorylation and functions of the RNA polymerase II CTD. Genes Dev, 2006. 20(21): p. 2922-36.

252. Palancade, B. and O. Bensaude, Investigating RNA polymerase II carboxyl-terminal domain (CTD) phosphorylation. Eur J Biochem, 2003. 270(19): p. 3859-70.

253. Solow, S., et al., Taf(II) 250 phosphorylates human transcription factor IIA on serine residues important for TBP binding and transcription activity. J Biol Chem, 2001. 276(19): p. 15886-92.

254. Kollmann, K., et al., A Kinase-Independent Function of CDK6 Links the Cell Cycle to Tumor Angiogenesis. Cancer Cell, 2013. 24(2): p. 167-181.

255. Bellutti, F., et al., CDK6 Antagonizes p53-Induced Responses during Tumorigenesis. Cancer Discov, 2018. 8(7): p. 884-897.

256. Bienvenu, F., et al., Transcriptional role of cyclin D1 in development revealed by a "geneticproteomic" screen. Nature, 2010. 463(7279): p. 374-8.

257. Casimiro, M.C., et al., ChIP sequencing of cyclin D1 reveals a transcriptional role in chromosomal instability in mice. The Journal of Clinical Investigation, 2012. 122(3): p. 833-843.

258. Malhotra, V. and M.C. Perry, Classical chemotherapy: mechanisms, toxicities and the therapeutic window. Cancer Biol Ther, 2003. 2(4 Suppl 1): p. S2-4.

259. Secchiero, P., et al., TNF-related apoptosis-inducing ligand (TRAIL): a potential candidate for combined treatment of hematological malignancies. Curr Pharm Des, 2004. 10(29): p. 3673-81.

260. Kranz, D. and M. Dobbelstein, A killer promoting survival: $p 53$ as a selective means to avoid side effects of chemotherapy. Cell Cycle, 2012. 11(11): p. 2053-4.

261. Carrillo, A.M., et al., Pharmacologically Increasing Mdm2 Inhibits DNA Repair and Cooperates with Genotoxic Agents to Kill p53-Inactivated Ovarian Cancer Cells. Mol Cancer Res, 2015. 13(8): p. 1197-205. 
262. Wang, S., et al., SAR405838: an optimized inhibitor of MDM2-p53 interaction that induces complete and durable tumor regression. Cancer Res, 2014. 74(20): p. 5855-65.

263. Andreeff, M., et al., Results of the Phase I Trial of RG7112, a Small-Molecule MDM2 Antagonist in Leukemia. Clin Cancer Res, 2016. 22(4): p. 868-76.

264. Sun, D., et al., Discovery of AMG 232, a Potent, Selective, and Orally Bioavailable MDM2-p53 Inhibitor in Clinical Development. Journal of Medicinal Chemistry, 2014. 57(4): p. 1454-1472.

265. DiNardo, C.D., et al., Phase 1 Dose Escalation Study of MDM2 Inhibitor DS-3032b in Patients with Hematological Malignancies - Preliminary Results. Blood, 2016. 128(22): p. 593-593.

266. Furet, P., et al., Discovery of a novel class of highly potent inhibitors of the p53-MDM2 interaction by structure-based design starting from a conformational argument. Bioorg Med Chem Lett, 2016. 26(19): p. 4837-4841.

267. Kojima, K., et al., The novel tryptamine derivative JNJ-26854165 induces wild-type p53- and E2F1-mediated apoptosis in acute myeloid and lymphoid leukemias. Mol Cancer Ther, 2010. 9(9): p. 2545-57.

268. Roxburgh, P., et al., Small molecules that bind the Mdm2 RING stabilize and activate p53. Carcinogenesis, 2012. 33(4): p. 791-8.

269. Furukawa, H., et al., PRIMA-1 induces p53-mediated apoptosis by upregulating Noxa in esophageal squamous cell carcinoma with TP53 missense mutation. Cancer Sci, 2018. 109(2): p. $412-421$.

270. Lambert, J.M., et al., PRIMA-1 reactivates mutant $p 53$ by covalent binding to the core domain. Cancer Cell, 2009. 15(5): p. 376-88.

271. Boeckler, F.M., et al., Targeted rescue of a destabilized mutant of p53 by an \&lt;em\&gt;in silico\&lt;/em\&gt; screened drug. Proceedings of the National Academy of Sciences, 2008. 105(30): p. 10360

272. Friedler, A., et al., A peptide that binds and stabilizes p53 core domain: Chaperone strategy for rescue of oncogenic mutants. Proceedings of the National Academy of Sciences, 2002. 99(2): p. 937.

273. Yu, X., et al., Allele-Specific p53 Mutant Reactivation. Cancer Cell, 2012. 21(5): p. 614-625.

274. Deneberg, S., et al., An open-label phase I dose-finding study of APR-246 in hematological malignancies. Blood Cancer Journal, 2016. 6: p. e447.

275. Silva, J.L., et al., Prion-like aggregation of mutant p53 in cancer. Trends Biochem Sci, 2014. 39(6): p. 260-7.

276. Soragni, A., et al., A Designed Inhibitor of p53 Aggregation Rescues p53 Tumor Suppression in Ovarian Carcinomas. Cancer Cell, 2016. 29(1): p. 90-103.

277. Alexandrova, E.M., et al., Improving survival by exploiting tumour dependence on stabilized mutant p53 for treatment. Nature, 2015. 523(7560): p. 352-6.

278. Parrales, A. and T. Iwakuma, Targeting Oncogenic Mutant p53 for Cancer Therapy. Front Oncol, 2015. 5.

279. Matsusaka, H., et al., Targeted deletion of p53 prevents cardiac rupture after myocardial infarction in mice. Cardiovasc Res, 2006. 70(3): p. 457-65.

280. Stempien-Otero, A., et al., Mechanisms of hypoxia-induced endothelial cell death. Role of p53 in apoptosis. J Biol Chem, 1999. 274(12): p. 8039-45.

281. Graeber, T.G., et al., Hypoxia-mediated selection of cells with diminished apoptotic potential in solid tumours. Nature, 1996. 379(6560): p. 88-91.

282. Hu, W., et al., Mechanistic Investigation of Bone Marrow Suppression Associated with Palbociclib and its Differentiation from Cytotoxic Chemotherapies. Clin Cancer Res, 2016. 22(8): p. 2000-8. 


\title{
10. CURRICULUM VITAE
}

\author{
ANUSHA SRIRAMAN \\ I) PERSONAL DETAILS
}

Work: Institute for Molecular Oncology

Justus von Liebig Weg 11

Göttingen Germany 37077

asriram@gwdg.de

Phone number: 0049-15211841314

Home: 17, Theodor Heuss Str. 26

Göttingen Germany 37075

Born: 15th October 1991

Nationality: Indian

\section{II) EDUCATION}

\section{Doctor of Philosophy (Ph.D.)}

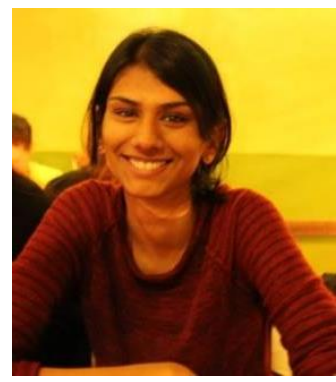

Göttingen Graduate School for Neurosciences, Biophysics, and Molecular Biosciences (GGNB), Institute of Molecular Oncology, University Medical Center Göttingen, Göttingen, Germany

Supervisor: Prof. Dr. Matthias Dobbelstein

Thesis title: Targeting MDM2, the antagonist of the tumor suppressor p53

The main aim of my doctoral thesis is to fortify $p 53$, a well-known tumor suppressor referred to as the 'guardian of our genome', in p53 proficient cancers by inactivating its antagonist MDM2 using inhibitors in combination with other drugs. 
Göttingen ,University Medical Center Göttingen, Georg-August Universität Göttingen

Thesis title: Mechanism of chronic obstructive pulmonary disorders (COPD) in different murine models (Supervisor: Dr Muriel Lizé, Department of Molecular and Experimental Pneumology, Göttingen)

Analysis of COPD in TAp73 knock-out mice lung was carried out by Sirius Red/Fast Green staining and histology. A possible connection between TAp73 and FoxJ1, the master regulator of ciliogenesis was identified.

Final examination 2,09

\section{Bachelor of Engineering, Biotechnology (B.E.)}

$2009-2013$

M.S. Ramaiah Institute of Technology, Karnataka, India

Thesis title: Reduction in motility of S. Typhimurium by curcumin (Supervisor: Prof. Dipshikha Chakravortty, Indian Institute of Science, Bangalore)

Curcumin, a food additive common in cooking, was found to decrease the motility of Salmonella enterica serovar Typhimurium, making the flagella smaller and more fragile.

Final examination - cgpa $9.6 / 10$

\section{III) PUBLICATIONS}

1. Anusha Sriraman, Antje Dickmanns, Zeynab Najafova, Steven Johnsen, Matthias Dobbelstein. CDK4 inhibition diminishes p53-activation by MDM2 antagonists. Manuscript under consideration at Cell Death and Disease.

2. Anusha Sriraman, Yizhu Li, Matthias Dobbelstein. Fortifying p53 - beyond Mdm2 inhibitors. Aging (Albany, NY) (2016). 2016 Sep 29. doi: 10.18632/aging.101073. [Epub ahead of print]

3. Alice Nemajerova, Daniela Kramer, Saul S Siller, Christian Herr, Orr Shomroni, Tonatiuh Pena, Cristina Gallinas Suazo, Katharina Glaser, Merit Wildung, Henrik Steffen, Anusha Sriraman, Fabian Oberle, Magdalena Wienken, Magali Hennion, Ramon Vidal, Bettina Royen, Mihai Alevra, Detlev Schild, Robert Bals, Jürgen Dönitz, Dietmar Riedel, Stefan Bonn, Ken-Ichi Takemaru, Ute M Moll, Muriel Lize. TAp73 is a central transcriptional regulator of airway multiciliogenesis. Genes and Development (2016). doi: 10.1101/gad.279836.116

4. Anusha Sriraman, Marija Radovanovic, Magdalena Wienken, Zeynab Najafova, Yizhu Li*, and Matthias Dobbelstein*. Cooperation of Nutlin-3a with a Wip inhibitor to induce p53 activity. Oncotarget (2016). 10.18632/oncotarget.9302

5. Yizhu Li*, Priyanka Saini*, Anusha Sriraman*, Matthias Dobbelstein. Mdmz inhibition confers protection of p53-proficient cells from the cytotoxic effects of Wee1 inhibitors. Oncotarget (2015). 10.18632/oncotarget.5891 *equal first authors 
6. Marouane Libiad, Anusha Sriraman, Ruma Banerjee. Polymorphic variants of human rhodanese exhibit differences in thermal stability and sulfurtransfer kinetics. J. Biol. Chem. (2015). 10.1074/jbc.M115.675694

IV) ACADEMIC ACHIEVEMENTS

Molecular Medicine, at the Georg-August University of $10 / 2013-09 / 2014$ Goettingen, Germany, with scholarship from the Federal Government of Lower Saxony

All India Rank 18 (AIR 18) with 99.95 percentile in the Graduate $03 / 2013-04 / 2014$ Aptitude Test for Engineering 2013 (GATE) conducted by Indian Institute of Technology Bombay, Mumbai in the Biotechnology stream

"Best Achiever 2013" in academics and co-curricular activities at M.S Ramaiah Institute of Technology, Bangalore, India

09/2009-08/2013

Silver medalist in the examinations conducted by Visvesvaraya Technological University, Belgaum, India.

$09 / 2009-08 / 2013$

Summer Research Fellowship by the Indian Academy of Sciences

$06 / 2012-08 / 2012$

V) TEACHING AND SUPERVISION

Instruction of 4 day methods course for Masters students of the $10 / 2015,10 / 2016$ IMPRS Molecular Biology on 'DNA techniques' including DNA fiber assay, RNA isolation, CDNA synthesis and quantitativeRT PCR

Supervision of two masters students during their lab rotation

$07 / 2016,05 / 2017$

Conducted interactive tutorials for Masters students of the IMPRS Molecular Biology program

$10 / 2016,10 / 2017$

VI) INTERNSHIPS

Department of Neurobiology, Max Planck Institute for 07/2014-09/2014 Experimental Medicine, Goettingen, Germany. Supervisors: Dr Jeong Seop Rhee and Prof. Nils Brose

Department of Biological Chemistry, University of Michigan, Ann Arbor, USA. Supervisor: Prof. Ruma Banerjee

$04 / 2014-05 / 2014$ Institute of Molecular Oncology, University of Goettingen, Germany. Supervisor: Dr Muriel Lizé 
Department of Cancer Biology, Indian Institute of Technology

Madras, Chennai, India. Supervisor: Prof. Devarajan Karunagaran

Division of Biological Sciences, Indian Institute of Science Bangalore, India. Supervisor; Prof. Dr. Dipshikha Chakravortty

$06 / 2011-08 / 2011$

VII) CONFERENCES AND SYMPOSIUM

$8^{\text {th }}$ Molecular Medicine retreat for PhD students (Drübeck, $06 / 2018$ Germany) Oral presentation

EKFS symposium on 'Exploring tumor vulnerabilities for improved cancer treatment' (Göttingen, Germany) Poster presentation

$17^{\text {th }}$ International p53 workshop (Singapore, Singapore) Poster presentation

$07 / 2017$

$7^{\text {th }}$ Molecular Medicine retreat for PhD students (Wernigerode, Germany) Oral presentation

$19^{\text {th }}$ International AEK congress (Heidelberg, Germany) Poster 03/2017 presentation

$32^{\text {nd }}$ Ernst Klenk symposium on 'Precision Oncology: Translating $12 / 2016$ basic discoveries into patient survival' (Cologne, Germany) Poster presentation 